\title{
The Effect of Statistical Wind Corrections on Global Wave Forecasts
}

Thomas Hawkins Durrant

Submitted in total fulfilment of the requirements

of the degree of Doctor of Philosophy

November 2011

School of Earth Sciences

The University of Melbourne 


\section{Abstract}

The ability to forecast ocean waves relies to a large extent on numerical models. Current third generation wave models have been found by many studies to produce highly accurate forecasts several days in advance. The skill of these models is such that the quality of the wave forecast is highly dependent on errors in the forcing wind field. On global scales, a lack of wind and wave observations has historically hampered efforts to separate large scale systematic error due to inherent wave model deficiencies from that imparted by the forcing winds. The advent of remotely sensed observations from altimetry, and more recently scatterometry, provide high quality observations on the open ocean, allowing the spatial structure of the systematic error in both modelled fields to be quantified.

In this study, surface winds from the Australian Community Climate Earth System Simulator (ACCESS), the recently implemented operational atmospheric model at the Australian Bureau of Meteorology, are used as forcing for the WAVEWATCH III ${ }^{\circledR}$ wave model. A number of global wave hindcasts are performed over a four month period from July to October 2008. The geographical variation of systematic error in the surface winds and resulting modelled Significant Wave Height $\left(H_{s}\right)$ are then assessed using QuikSCAT scatterometer data and Jason-1 and Envisat altimeter data respectively.

A negative bias in the modelled $H_{s}$ is identified over most of the globe. The cause of this bias is determined to be largely due to a negative bias in the ACCESS winds. Subsequent to this finding, a number of means of statistically correcting the winds are explored. A simple correction over the entire domain is found to inadequately account for geographical variation in the wind bias. This is addressed by considering corrections that vary in space. Finally, these spatially varying corrections are extended to vary in time. In an operational environment, the error characteristics of the wind forcing can be expecting to change over time with the evolution of the atmospheric model. This in turn requires any applied 
correction to be monitored and maintained. Motivated by a desire to avoid this manual maintenance, a self learning correction method is proposed whereby spatially and temporally varying corrections are calculated in real time from a moving window of historical comparisons between observations and preceding forecasts. This technique is shown to effectively remove both global, and regionally varying wind speed biases.

Finally, the effect of these wind corrections on the modelled wave field is assessed. Large improvement is demonstrated in the Northern Hemisphere $H_{s}$, however, the applied corrections produce a positive bias in the Southern Hemisphere. Overall, it is clear that by correcting the winds, their contribution to the modelled $H_{s}$ error is reduced, allowing inherent wave model deficiencies to be more confidently isolated. 


\section{Declaration}

This is to certify that:

(i) the thesis comprises only my original work towards the $\mathrm{PhD}$ except where indicated,

(ii) due acknowledgement has been made in the text to all other material used,

(iii) the thesis is less than 100,000 words in length, exclusive of table, maps, bibliographies, appendices and footnotes.

Thomas Hawkins Durrant 


\section{Acknowledgments}

First and foremost, I would like to thank my Supervisors. To Diana Greenslade of the Bureau of Meteorology, as a supervisor, for her knowledge, her guidance, and her sense of humour; as a boss, for her patience and generosity, both with her time and in allowing me the time and freedom to balance the competing demands of my job with those of completing this thesis. To Ian Simmonds of the University of Melbourne, for his constant encouragement, trust and support, and his infectious love of science.

I would also like to thank Frank Woodcock. Frank gave me my first job at the Bureau. What was initially a possibly unwise 6 month hiatus from the work of my $\mathrm{PhD}$, has ended up significantly influencing it. His ongoing friendship, support and wisdom has been a valuable aside.

I would like to acknowledge and thank my employer, the Bureau of Meteorology for their financial assistance as well the ongoing generous study leave that has allowed my to complete this work on a part time basis.

A number of people have contributed to technical aspects of this work. I would like to extend my gratitude to Jean-Michel Lefevre (Meteo-France), and the European Space Agency for providing some Jason-1 and Envisat data respectively and Graham Warren and Mikhail Entel for help retrieving archived data at the Bureau. Technical support provided in the context of the Bureau's supercomputer environment from Tim Pugh and Justin Freeman was greatly appreciated. To Hendrik Tolman and Arun Chawla for kindly supplying the WAVEWATCH III ${ }^{\circledR}$ source code prior to the official release of version 3.14. Comments made on various parts of the manuscript by Eric Schulz, Jeff Kepert, Mark Hemer and Paul Sandery were also greatly appreciated.

There are many people within the Earth Science Department at the University of Melbourne that have helped keep me on track over the years. I would 
like to thank my honours supervisor, Alex Pezza both for providing me with the positive introduction to the world of academic research that inspired me to continue, and for his ongoing friendship. Todd Lane has provided much sound and balanced advice, both in relation to this work, and beyond. Whilst I was a little scarce in the Earth Science department, the friendship and camaraderie of my fellow PhD students has been a greatly valued aspect of this journey. Thank you to Nick Summonds, Prasanth Divakaran, Elise Chandler, Tom Fromhold, Cam Watson, Julie Arblaster, Claire Fenby, Dragana Zovko Rajak and Jill Lynch, amongst many others. Malek Ghantous also falls into this catagory, and is singled out here due to the distinguishing quality of also having an interest in ocean waves, from which many interesting conversations have ensued. I also owe much gratitude to my office mates at the Bureau, Arthur Simanjuntak and Stewart Allen, who coincidentally have both shared my experiences as part time $\mathrm{PhD}$ students.

On a more personal note, I could not have completed this thesis without the support of my family and friends. To my family, for the enormous support that they have given and continue to give me, not only throughout my PhD, but but in all the years that preceded it. To my friends, for their quiet support, for knowing not to ask how it is going, and for remembering me after a prolonged period of seclusion on my part. In particular, to Kasimir Stokes Burgess, I could not wish for a more supportive and understanding friend.

Finally, to Celeste. For her love, her support, and her enormous patience. For feeding me, for looking after me, and in these last few months, for her seemingly limitless ability to put up with me. I cannot thank her enough. 


\section{Contents}

1 Introduction $\quad 1$

1.1 Introduction . . . . . . . . . . . . . . . . . . 1

1.2 Background ........................ . . 3

1.2.1 Numerical Wave Modelling . . . . . . . . . . . . . . . . 3

1.2.2 Wave Model Validation . . . . . . . . . . . . . . . 5

1.2.3 Sensitivity to the Accuracy of the Forcing Winds . . . . . 6

1.2.4 Statistical Wind Corrections . . . . . . . . . . . . . . 9

1.2.5 Implications for Operational Forecasting . . . . . . . . . 11

1.3 Aim of the Present Study . . . . . . . . . . . . . . . . . 12

1.4 Models Used in this Study . . . . . . . . . . . . . . . . . 13

1.5 Structure of Thesis . . . . . . . . . . . . . . . 13

2 Validation of Jason-1 and Envisat Significant Wave Height 15

2.1 Introduction . . . . . . . . . . . . . . . . . . . . 15

2.2 Previous Work . . . . . . . . . . . . . . . . 16

2.2 .1 Jason-1 . . . . . . . . . . . . . . . . . . . . . . . 18

2.2 .2 Envisat . . . . . . . . . . . . . . . 19

2.3 Method ......................... 20

2.3 .1 Data . . . . . . . . . . . . . . 20

2.3 .2 Method ...................... . . 21

2.4 Results . . . . . . . . . . . . . . . . . . . 23

2.4.1 MEDS and NDBC buoy network differences . . . . . . . 23

2.4 .2 Validation Results . . . . . . . . . . . . . . 27

2.4 .3 Wave State Influence . . . . . . . . . . . . . . . . . . . 30

2.5 Summary . . . . . . . . . . . . . . . . . 34 
3 An Assessment of the ACCESS Forcing Winds 35

3.1 Introduction . . . . . . . . . . . . . . . . 35

3.2 Previous Work . . . . . . . . . . . . . 36

3.3 Data . . . . . . . . . . . . . . . . 37

3.4 Method ........................ 40

3.5 Results ........................... 44

3.6 Summary . . . . . . . . . . . . . . . . . 52

4 Set Up and Verification of WAVEWATCH III 53

4.1 Introduction . . . . . . . . . . . . . . . . . 53

4.2 The Wave Model . . . . . . . . . . . . . . . . . . . 54

4.3 Data and Method . . . . . . . . . . . . . . 58

4.3 .1 Model Set Up . . . . . . . . . . . . . . . 58

4.3 .2 Model Runs . . . . . . . . . . . . . . . . 60

4.3 .3 Verification Method .............. 61

4.4 Results . . . . . . . . . . . . . . . . 66 66

4.4 Source Terms . . . . . . . . . . . . . 66

4.4 .2 Numerics . . . . . . . . . . . . . . . . 72

4.4.3 Sub-Grid-Scale Blocking . . . . . . . . . . . 76

4.5 Summary . . . . . . . . . . . . . . . . . . . . 79

5 Statistical Wind Corrections $\quad 81$

5.1 Introduction . . . . . . . . . . . . . . . . 81

5.2 Background ........................... 82

5.3 Method ....................... 85

5.4 Results .......................... 87

5.4.1 Static and Homogeneous . . . . . . . . . . . 87

5.4 .2 Spatially Varying Corrections . . . . . . . . . . . . . 88

5.4.3 Spatially and Temporally Varying Learned Corrections . . 95

5.5 Discussion . . . . . . . . . . . . . . . . 106

5.6 Conclusions . . . . . . . . . . . . . . . . . . . 109

6 The Effect of Wind Corrections on the Wave Field 113

6.1 Introduction . . . . . . . . . . . . . . . . . 113 
6.2 Method . . . . . . . . . . . . . . . . . 114

6.3 Global Sensitivity of the Wave Field to the Forcing Winds . . . . 117

6.4 Results for BAJ Source Terms . . . . . . . . . . . . . 120

6.4.1 Qualatitive Attribution of Large Scale Systematic Error . . 121

6.4.2 Quantitative Effect of Statistical Wind Corrections on the Modelled Wave Field . . . . . . . . . . . . . . . . 127

6.4.3 Residual Systematic Wave Bias . . . . . . . . . . . . 131

6.5 Comparative Results for TC96 Source Terms . . . . . . . . . . . . 141

6.6 Discussion . . . . . . . . . . . . . . . . . 146

6.6.1 Simple Source Term Tuning . . . . . . . . . . . . 147

6.7 Summary and Conclusions . . . . . . . . . . . . . . . . 152

7 Summary and Conclusions $\quad 155$

7.1 Summary and Key Findings: . . . . . . . . . . . . . . . . . . . 155

7.2 Further Work . . . . . . . . . . . . . . . . . . . . 159

7.3 Closing Remarks . . . . . . . . . . . . . . . . . 162

$\begin{array}{ll}\text { References } & 163\end{array}$

$\begin{array}{ll}\text { Appendices } & 177\end{array}$

$\begin{array}{lr}\text { A LIST OF ACRONYMS } & 179\end{array}$

B Sensitivity to Differing Definitions of Altimeter-Buoy Spatial $\begin{array}{ll}\text { Proximity } & 183\end{array}$

$\begin{array}{lr}\text { C Buoy Details } & 189\end{array}$ 


\section{List of Tables}

2.1 Validation statistics for raw and linearly corrected data based on NDBC buoy comparisons for both Jason-1 and Envisat. For linearly corrected data, regression coefficients are given based on the relationship $H_{s}^{\text {corrected }}=\alpha H_{s}^{\text {raw }}+\beta \ldots \ldots \ldots \ldots$

2.2 Correlations between altimeter $H_{s}$ bias (altimeter minus buoy) and both $H_{s}$ and wave steepness as well as partial correlations for each variable with the effects of the other removed. . . . . . . . .

3.1 ACCESS $U_{10}$ verification statistics against QuikSCAT data for the period July to October 2008. . . . . . . . . . . . . . . . 44

3.2 ACCESS $U_{10}$ verification statistics against QuikSCAT data for each month of the period examined based on comparisons with QuikSCAT data. . . . . . . . . . . . . . . . .

4.1 Hindcasts performed and analysed in this chapter. . . . . . . . .

5.1 Global verification statistics for ACCESS $U_{10}$ against QuikSCAT data before and after the application of homogeneous corrections calculated retrospectively over the entire four month period. . .

5.2 Global verification statistics for ACCESS $U_{10}$ against QuikSCAT data before and after the application of spatially varying corrections calculated retrospectively over the entire four month period.

5.3 Global verification statistics for ACCESS $U_{10}$ against QuikSCAT data before and after the application of spatially and temporally varying learned corrections.

5.4 Regional statistics for $U_{10}$ relative to scatterometer data. $\%$ improvement refers to \% improvement in RMSE. . . . . . . . . . . 107 
6.1 Regional statistics for $H_{s}$ relative to altimeter data for BAJ source terms when forced with uncorrected winds as well as those corrected with a static homogeneous correction and spatially and temporally varying learned corrections. \% improvement refers to \% improvement in RMSE. . . . . . . . . . . . . . . . . . 128

6.2 Regional statistics for $H_{s}$ relative to altimeter data for TC96 source terms when forced with uncorrected winds as well as those corrected with a static homogeneous correction and spatially and temporally varying learned corrections. $\%$ improvement refers to $\%$ improvement in RMSE. . . . . . . . . . . . . . . . . . . . . . 143

6.3 Regional statistics for $H_{s}$ relative to altimeter data for BAJ terms with uncorrected winds and the default setting for the $\beta_{\max }$ tuning parameter (1.2), with uncorrected winds and increased $\beta_{\max }$ (1.6) and with spatially and temporally varying learned corrections applied to the wind and a reduced $\beta_{\max }$ (1.13). \% improvement refers to \% improvement in RMSE. . . . . . . . . . . . . . . . . . . . . . 150

B.1 RMS difference $(\mathrm{m})$ of altimeter data relative to buoy data for buoys above and below $45^{\circ}$ based on passes gathered using $1 \times 1^{\circ}$ boxes and $50 \mathrm{~km}$ radius circles . . . . . . . . . . . . . . 184

C.1 Wave buoys located around the Australian coast. Those used for verification are shown in bold. . . . . . . . . . . . . . . . . 189 


\section{List of Figures}

2.1 Altimeter ground tracks from one day for Jason-1 (red) and Envisat

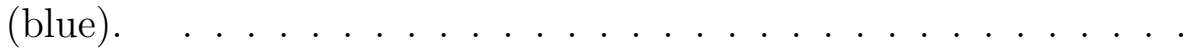

2.2 Buoys used in the altimeter validation. NDBC are marked with squares, MEDS buoys are marked with triangles . . . . . . . . .

2.3 Scatter plots of co-located $H_{s}$ observations for Jason-1 and Envisat for both the NDBC and MEDS buoy networks separately. Panels on the top ( $\mathrm{a}$ and $\mathrm{b}$ ) show Jason-1 data while those on the bottom (c and d) show Envisat data. Panels on the left (a and c) show colocations with NDBC buoys only, those on the right (b and d) show co-locations with MEDS buoys only. The number of co-locations in each $0.5 \mathrm{~m}$ bin have have been contoured . . . . . . . . . . . .

2.4 (a) Co-located Jason-1 and buoy data and (b) the same data with the number of co-locations in each $0.5 \mathrm{~m}$ bin contoured. (c) and (d) show the same for Envisat data . . . . . . . . . . . .

2.5 Altimeter bias as a function of wave steepness. The size of the ellipses gives the number of co-locations in each bin . . . . . .

2.6 Mean $H_{s}$ as a function of wave steepness. The size of the ellipses gives the number of co-locations in each bin . . . . . . . . .

2.7 Altimeter bias as a function of (a) $H_{s}$ and (b) wave steepness for both corrected and uncorrected altimeter data (note that the uncorrected data in (b) is the same as that of Figure 2.5). . . . .

3.1 One day of QuikSCAT data showing observed $U_{10}$ in $\mathrm{ms}^{-1}$. . . .

3.2 Example of $U_{10}$ co-locations. QuikSCAT pass before (a) and after (b) removal of poor quality data, model data for 4 July 2008 (a) and the magnitude of the difference between co-locations calculated for this pass (d). All plots in units of $m s^{-1}$. . . . . . . . . . .

3.3 Number of QuikSCAT-model co-locations in each $1 \times 1^{\circ}$ bin. . .

3.4 Scatter plot of $U_{10}$ based on comparisons with QuikSCAT data for the period July to October 2008. Data are density in $0.5 \times 0.5 \mathrm{~m}$ bins are shown on a logarithmic scale. . . . . . . . . . 
3.5 Probability density function for modelled and observed wind speeds.

3.6 $U_{10}$ statistics based on QuikSCAT data for the period July to October 2008. . . . . . . . . . . . . . . . . . . 47

3.7 ACCESS $U_{10}$ bias and SI based on comparisons with QuikSCAT data. . . . . . . . . . . . . . . . . . . 48

3.8 Same as Figure 3.7 for the Australian east coast. . . . . . . . . . 49

3.9 ACCESS $U_{10}$ bias for each month over the period examined. . . . 51

3.10 ACCESS wind direction bias $\left(^{\circ}\right)$ based on comparisons with QuikSCAT data for the period July to October 2008. . . . . . . . . . . 52

4.1 Validations of bathymetry data sets at known buoy depths around the Australian coast. . . . . . . . . . . . . . . . 59

4.2 Bathymetry $(\mathrm{m})$ derived from DBDB2 version 3.0 for the global grid on a logarithmic depth scale. . . . . . . . . . . . . 60

4.3 Australian buoys used in this study. Buoy groups mentioned in the verification statistics are indicated. . . . . . . . . . 62

4.4 Crowdy Head (55019) $H_{s}$ buoy data for the month of July 2008, with observations removed by automatic quality control shown in red. . . . . . . . . . . . . . . .

4.5 Jason-1 (red) and Envisat (blue) data over the Tasman Sea for $10^{\text {th }}$ July 2008. (a) shows all data with those removed through the quality control checks shown in black, and (b) super-obs calculated from the remaining data. . . . . . . . . . . . . . . 64

4.6 Number of altimeter-model $H_{s}$ co-locations in each $3 \times 3^{\circ}$ bin. . . 65

4.7 $H_{s}$ statistics based on altimeter data for the period July to October $2008 \ldots \ldots \ldots \ldots \ldots \ldots \ldots$

4.8 Overall $H_{s}$ statistics for BAJ, WAM3 and TC96 source term packages relative to buoys shown in figure 4.3 and global altimeter data. 68

4.9 Spatial plots of $H_{s}$ bias (left hand column) and SI (right hand column) for TC96, BAJ and WAM3 source terms calculated against Jason-1 and Envisat data. . . . . . . . . . . . . . . . . . 69

4.10 Bias and SI for $T_{p}$ for different source term options. . . . . . . . . 72

4.11 Time series of $H_{s}$ (a) and $T_{p}$ (b) for both the UQ and $1_{s t}$ order numerical schemes at the Cape du Couedic waverider buoy for the month of July 2008. . . . . . . . . . . . . . . . .

4.12 Statistics for 1 st order and UQ numerical schemes relative to buoy and altimeter data. . . . . . . . . . . . . . . . . . 74 
4.13 Mean difference $(\mathrm{m})$ between $H_{s}$ from the 1st order and UQ numerical schemes $\left(\mathrm{UQ}-1_{s t}\right) . \ldots \ldots . \ldots 75$

4.14 Percentage improvement in $H_{s}$ SI for UQ over the first order scheme $\left(\left(S I_{1 s t}-\right.\right.$ $\left.\left.S I_{U Q}\right) / S I_{1 s t} \times 100\right) \ldots \ldots \ldots \ldots \ldots 76$

$4.15 H_{s}(\mathrm{~m})$ snapshots for a wave system propagating northward through French Polynesia using BAJ source terms and UQ numerics with (a) and without (b) sub-grid-scale blocking activated and the difference between the two $(\mathrm{c}) . \ldots \ldots \ldots$. . . . . . . .

4.16 $H_{s}$ spatial bias $(\mathrm{m})$ and SI for BAJ with (a, c) and without (b, d) sub-grid-scale blocking activated. Both runs use UQ numerics. . .

5.1 Spatial $U_{10}$ bias after increasing $U_{10}$ uniformly by $6 \%$. . . . . . .

5.2 Regions for which wind roses and directional dependence of slope are calculated. See Figure 5.3. . . . . . . . . . . .

5.3 For each region shown in figure 5.2, the left panels show model wind roses, the middle panels show observation wind roses and the right panels show the directionally dependent slope correction, constructed by binning co-locations into $20^{\circ}$ directional bins according to observation direction, and calculating the slope correction for each bin. . . . . . . . . . . . . . . . . . . . . . 91

5.3 Continued. . . . . . . . . . . . . . . . . . . . 92

5.4 Spatially varying correction coefficients, calculated independently for each $1 \times 1^{\circ}$ model grid point. . . . . . . . . . . . . .

$5.5 U_{10}$ bias after applying the spatially variant slope correction shown in Figure 5.4 (b)

5.6 Schematic of the learned correction method. $w$ represents an arbitrary learning window. Time steps of 3 hours used in this work, with with the calculated correction varying geographically well as time, see text for details. . . . . . . . . . . . . . . .

5.7 Correction grids calculated for July $1^{\text {st }} 2008$ for given learning window (days) . . . . . . . . . . . . . . .

5.8 The total number of observations used to construct the spatial corrections in Figure $5.7 \ldots \ldots \ldots$. . . . . . . .

5.9 Correction grids for July $1^{\text {st }}$ using a 10 day learning period with no smoothing applied (a) and Gaussian smoothing filters applied of indicated sizes (b)-(c). Note that (a) is the same as Figure 5.7 (c)101

5.10 Percentage improvement in RMSE relative to scatterometer data for corrected wind fields over the uncorrected wind field for different length learning windows and different levels of smoothing applied to the calculated correction field. . . . . . . . . . . . 102 
5.11 As in Figure 5.10, but with operational timings. See text for details.

5.12 Calculated spatially and temporally varying learned slope correction at monthly intervals over the period of this study. Corrections shown here represent those applied at the model dates indicated, in each case, corrections are calculated from the 30 days prior to this date (see text for details). Corrections are calculated and applied every 3 hours, select grids are shown here at monthly intervals to demonstrate the evolution of the correction over the period examined in this work.

5.13 Bias of winds corrected with a 30 day learning window applied every 3 hours. . . . . . . . . . . . . . . . . . . 105

5.14 Percentage improvement in RMSE achieved by applying a learned slope correction with a window length of 30 days, and no spatial smoothing. . . . . . . . . . . . . . . . 106

5.15 PDFs of scatterometer observations and co-located modelled winds (left) and model errors (right) . . . . . . . . . . . . . . 108

5.16 Box and whisker plots of the differences between scatterometer observations and co-located model $U_{10}$. The center band of the box indicates the median, the top and bottom of the box represent the upper and lower quartiles respectively and the whiskers show the most extreme values within 1.5 times the inner quartile range.

6.1 Percentage increase in the mean $H_{s}$ resulting from a $6 \%$ increase in the forcing winds. . . . . . . . . . . . . . . . . . . . 118

6.2 As in Figure 6.1 but with corrections applied to the Northern Hemisphere extratopics only (a) the tropics only (b) and the Southern Hemisphere extratropics only $(\mathrm{c})$. . . . . . . . . . . . . . . 119

6.3 Bias over the whole 4 month period for $U_{10}$ (left column), and $H_{s}$ (right column) relative to scatterometer and altimeter data respectively for uncorrected ACCESS winds (first row), winds corrected using static homogeneous corrections (second row) and those corrected using spatially and temporally varying learned corrections (third row). Repeated figures include: (a) Figure 3.7 (a), (b) Figure 4.9 (e), (c) Figure 5.1 and (e) Figure 5.13. . . . . . . . . . 122

6.4 As figure 6.3, with Gaussian smoothing applied over 5 boxes in the case of the $1^{\circ} U_{10}$ bias, and 2 boxes for the $3^{\circ} H_{s}$ bias. . . . . . . 123

6.5 Percentage improvement in modelled Significant Wave Height $\left(H_{s}\right)$ RMSE achieved by correcting the forcing winds with spatially and temporally varying learned corrections using BAJ source terms. 
6.6 PDFs of altimeter observed $H_{s}$ and co-located modelled $H_{s}$ (left) and differences (right). . . . . . . . . . . . . . 130

6.7 Box and whisker plots of the differences between altimeter observations and co-located model $H_{s}$ for BAJ source terms, forcing with both uncorrected and corrected winds, using learned spatially and temporally varying corrections. The center band of the box indicates the median, the top and bottom of the box represent the upper and lower quartiles respectively and the whiskers show the most extreme values within 1.5 times the inner quartile range. A minimum of 100 co-locations is required in each bin. . . . . . . . .

6.8 Percentage of time over the four months examined here that the modelled $H_{s}$ values over $10 \mathrm{~m}$ and $U_{10}$ over $20 \mathrm{~ms}^{-1}$ occur simultaneously. . . . . . . . . . . . . . . . .

6.9 $H_{s}$ SI for BAJ runs with (a) uncorrected and (b) corrected winds. (a) is a repeat of Figure 4.9 (f) . . . . . . . . . . . 136

6.10 Taylor diagram showing $U_{10}$ statistics relative to QuikSCAT data for both uncorrected Australian Community Climate Earth System Simulator (ACCESS) winds, and those corrected using spatially and temporally varying learned corrections. . . . . . . . . . . . 137

6.11 Taylor diagram showing $H_{s}$ statistics relative to altimeter data when forced with both uncorrected ACCESS winds, and those corrected using spatially and temporally varying learned corrections.

$6.12 H_{s}$ bias and SI for TC96 run with uncorrected, and corrected winds. Figures (a) and (b) are repeats of Figures 4.9 (c) and (d) respectively. . . . . . . . . . . . . . . . . . . . . . . 142

6.13 Percentage improvement in modelled $H_{s}$ RMSE achieved by correcting the forcing winds with spatially and temporally varying learned corrections using TC96 source terms. . . . . . . . . . . . . 144

6.14 As Figure 6.7 for TC96 source terms . . . . . . . . . . . . . . 145

6.15 As Figure 6.11 for TC96 source terms. . . . . . . . . . . . . . 146

$6.16 H_{s}$ bias relative to altimeter data for BAJ terms with (a) uncorrected winds and the default setting for the $\beta_{\max }$ tuning parameter (1.2) (repeat of Figure 6.4 (b)), (b) uncorrected winds and increased $\beta_{\max }$ (1.6) and (c) with spatially and temporally varying learned corrections applied to the wind and a reduced $\beta_{\max }$ (1.13). 151 
B.1 Example of Jason-1 altimeter passes made over a buoy off Hawaii. Dotted grid lines are at $1^{\circ}$ intervals. The western end of the horizontal lines marks the altimeter ground track, and the line length gives the measured altimeter $H_{s}$. Julian day time of observation (t:), post processing altimeter observation (A:) and interpolated buoy observation (B:) resulting from each pass are shown . . . . 184

B.2 Ground tracks over a buoy located near $57^{\circ} \mathrm{N}$ for (a) Jason-1 and (b) Envisat presented in stereographic projection. Surrounding the buoy is a $1 \times 1^{\circ}$ box used to define spatial co-location limits (see

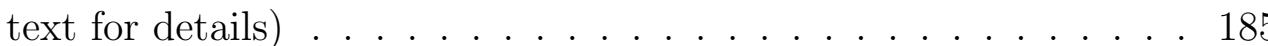

B.3 Number of valid co-locations arising from buoys at different latitudes for the described method using both $\times 1^{\circ}$ boxes and $50 \mathrm{~km}$ radius circles for both Jason-1 and Envisat . . . . . . . . . . 186 


\section{Chapter 1}

\section{Introduction}

\section{Contents}

1.1 Introduction $\ldots \ldots \ldots \ldots \ldots$

1.2 Background .................. 3

1.2.1 Numerical Wave Modelling . . . . . . . . . . . . . 3

1.2.2 Wave Model Validation . . . . . . . . . . . . 5

1.2.3 Sensitivity to the Accuracy of the Forcing Winds . . . 6

1.2.4 Statistical Wind Corrections _.......... . 9

1.2.5 Implications for Operational Forecasting . . . . . . . . 11

1.3 Aim of the Present Study _. . . . . . . . . 12

1.4 Models Used in this Study . . . . . . . . . . . 13

1.5 Structure of Thesis $\ldots \ldots \ldots \ldots$

\subsection{Introduction}

Ocean waves are almost always present at sea. These waves are generated by winds somewhere on the ocean, be it locally, so called wind sea, or thousands of kilometres away, referred to as swell. Ocean waves affect a wide range of activities such as shipping, fishing, recreations, coastal and offshore industry, coastal management and pollution control. They are also important in the climate processes as they play a large role in exchanges of heat, energy, gases and particles between the ocean and the atmosphere. 
The ability to forecast these waves relies to a large extent on numerical models. Current third generation wave models such as WAM (WAMDIG, 1988) and WAVEWATCH III ${ }^{\circledR}$ (WW3, Tolman, 1991, 2009) have been found by many studies to produce highly accurate forecasts several days in advance. The skill of these models is such that the quality of the wave forecast is, to a large extent, determined by errors in the forcing wind field (e.g. Cardone et al., 1996; Rogers and Wittmann, 2002). A 10 percent error in the estimate of surface wind speed can lead to 10-20 percent errors in $H_{s}$ and 20-50 percent errors in wave energy (Cavaleri, 1994).

Until recently, the operational wave model run at the Australian Bureau of Meteorology (Bureau) was the Australian WAve Model (AUSWAM), based on the widely used WAM model. One of the motivations for the research undertaken in this thesis arose from recent comparisons of AUSWAM against ERS-2 altimeter data that revealed a distinct spatial distribution of bias in modelled $H_{s}$ over the globe. In particular, a typically large negative bias at around 40-50 $\mathrm{S}$ globally was identified by Greenslade and Young (2004b). Subsequent evaluation of the Global ASsimilation and Prediction System (GASP) forcing winds against QuickSCAT scatterometer data revealed these to be similarly negatively biased (Kepert et al., 2005; Schulz et al., 2007) and likely contributing to the observed bias in the wave model. Indeed, applying statistical corrections to these winds resulted in improved verification results at buoy locations around the Australian coast (Greenslade et al., 2005). However, the wind evaluations of Kepert et al. (2005) and Schulz et al. (2007) did not resolve the spatial structure of the error in the forcing winds. So while a general statement about the overall bias could be made, the degree to which the observed spatial variation modelled $H_{s}$ bias could be attributed to the forcing winds remained an open question.

The Bureau has recently replaced all the existing operational Numerical Weather Prediction (NWP) systems (i.e. GASP (Seaman et al., 1995) and Limited Area Prediction System (LAPS) (Puri et al., 1998)) with the ACCESS system, which is based on the UK Met Office Unified Model/Variational Assimilation (UM/VAR) system (Rawlins et al., 2007). This has led to better skill in standard NWP skill scores (NMOC, 2010a), however, no direct analysis of marine surface winds has been carried out, and the error characteristics of these winds, and their potential contribution to systematic wave error, is unknown.

This thesis documents an exploration of the extent to which large scale sys- 
tematic biases in modelled waves from a modern third generation wave model can be attributed to the forcing winds. Remotely sensed observations of $10 \mathrm{~m}$ wind speed $\left(U_{10}\right)$ and $H_{s}$ are used to examine the spatial structure of the systematic error in both modelled fields. Based on the outcomes of this work, a number of means of correcting the wind field are also explored, and the effect of these corrections on the wave field analysed. This chapter introduces some background and presents the aims and objectives of this study.

\subsection{Background}

\subsubsection{Numerical Wave Modelling}

Wave modelling and computer generated wave forecasting began in the late 1950's (Sverdrup and Munk, 1947; Hubert, 1957). Initially, wave models considered only a single representative wave described with a representative height and period. However, it has long been understood that waves at sea represent a stochastic process, and that a single representative wave height does not adequately describe the wave field at sea. This led to the introduction of a spectral description of waves. Numerical spectral wave models are based on a form of the spectral waves transport equation:

$$
\frac{\partial F}{\partial t}+\nabla\left(c_{g} F\right)=S_{t o t}
$$

where $F(f, \theta)$ is the wave spectrum as a function of frequency $(f)$ and direction $(\theta)$ and $c_{g}$ is the group velocity. The left hand side of this equation represent the spatial and temporal evolution of the wave spectrum, and the right hand side represents sources and sinks of wave energy. In deep water, these consist of the input of wave energy by wind $\left(S_{i n}\right)$, nonlinear interactions between waves $\left(S_{n l}\right)$ and dissipation due to wave breaking or 'whitecapping' $\left(S_{d s}\right)$. The non-linear interactions represent the process that shifts energy between spectral components and does not change the total wave energy.

The critical role of the non-linear interactions in the evolution of the spectrum was identified in the JOint North Sea WAve Project (JONSWAP) experiments (Hasselmann et al., 1973). The development of wave models can broadly be categorised by the treatment of this term. Though theoretically well understood, 
the explicit computation of the nonlinear interactions is prohibitively expensive to evaluate in practical wave models. The first spectral wave models did not attempt to explicitly compute the non-linear interactions, with spectral shapes and energy growth rates simply prescribed for wind seas. Such models are referred to as first and second generation wave models, a comprehensive evaluation of which was conducted by the The Sea Wave Model intercomparison Project ( SWAMP Group, 1985). The shortcomings of these models led to the establishment of the WAM Group, whose mission it was to develop a model that would explicitly resolve the energy balance equation. This was achieved with the development of the discrete interaction approximation (DIA: Hasselmann et al., 1985), employed in so called third generation models. The WAM model (WAMDIG, 1988) became the first operationally feasible third generation wave model.

The wind input term up to WAM Cycle 3 (WAM3) is modelled after Snyder et al. (1981), based on Miles' (1957) theory in which waves are generated by resonance between the wind-induced pressure waves at the water surface and propagating water waves. This theory was extended by Janssen (1991) to include a two way wave-atmosphere coupling, forming the basis for the WAM Cycle 4 (WAM4) input term.

Hasselmann (1974) proposed a formulation for the white-capping dissipation considering that the process could be treated as a random distribution of pressure pulses. This formed the basis of the WAM3 dissipation term, with the calibrations of Komen et al. (1984) based on the ability of the numerical model to match observed wave conditions. Based on the increase in the high frequency input from the Janssen (1991) term, a corresponding adjustment was required to the dissipation term used in WAM4 (Janssen et al., 1994). Further adjustments were made to the dissipation terms in the WAM model run at the European Centre for Medium-Range Weather Forecasts (ECMWF) by Bidlot et al. (2007).

Another widely used set of source term formulations are those of Tolman and Chalikov (1996), the current default terms in the WW3 model. The wind input source term is based on numerical predictions of air flow over waves (Chalikov and Belevich, 1993; Chalikov, 1995). Dissipation is handled with two distinct formulations employed at high and low frequencies, with a transition at two times the wind sea peak frequency. This allows the separation of physical processes contributed to dissipation at these respective scales. Unlike WAM variant terms, swell dissipation is notably accounted for in the TC96 terms, in the form of a 
negative wind input for waves travelling faster than, or at large angles to the wind. The importance of swell dissipation on global scales, originally determined heuristically in this case (Tolman, 2002a), has subsequently been more explicitly defined in the work of Ardhuin et al. (2010), based on observed swell decay rates (Collard et al., 2008).

A detailed description of these source terms is not given here. In addition to the referred papers above, the relevant descriptions of the physics of wave generation and dissipation, and their representation in modern source terms, can be found in the reviews of WAMDIG (1988) and Komen et al. (1996), with more up to date details of recent advances summarised in WISE Group (2007).

\subsubsection{Wave Model Validation}

The source terms applied to the evolution of the wave spectrum, though physically based, contain a number of tunable parameters. In general, the determination of tuning parameters is first performed based on their ability to reproduce simple idealised cases, such as known duration and fetch limited growth curves (e.g. Komen et al., 1984; SWAMP Group, 1985; Tolman and Chalikov, 1996). These tunings are then refined according to their ability to reproduce realistic wave fields in variable wind conditions. This is done through their application in large scale wave models used to simulate real world conditions, for which observations exist (e.g. Komen et al., 1996; Tolman, 2002a; Tolman et al., 2002; Bidlot et al., 2002, 2007; Ardhuin et al., 2008, 2010).

This process relies on the availability of high quality wave observations. Historically, data coverage over the ocean has been poor. For research applications, many studies have benefited from highly instrumented measurement campaigns such as JONSWAP (Hasselmann et al., 1973) and the Surface Wave Dynamics Experiment (SWADE, Weller et al., 1991). In operational applications, throughout the formative years of the WAM model, available wave data was primarily from moored buoys. By nature, these observations are few, located in selected areas, generally along coastlines, and can only produce local error estimates. Additionally, these data have historically come from the major North American and European buoy networks, potentially biasing the tuning of these models to conditions in the Northern Hemisphere.

The advent of satellite altimeter observations provided a boon for wave model 
verification and tuning; for the first time, the open ocean could be reliably observed. In contrast to the WAM source terms, the Tolman and Chalikov (1996) terms in WW3 relied heavily on altimeter data during developmental tuning (Tolman, 2002a; Tolman et al., 2002). Significant adjustments were required to the idealised tunings in order to achieve realistic wave fields on a global scale. It was found, for example, that swell dissipation due to opposing or weak winds was overestimated, requiring significant retuning. Additionally the model tuned in the classical way to fetch-limited growth for stable conditions was found to underestimate deep-ocean wave growth requiring a subsequent retuning for effects of atmospheric stability (Tolman, 2002a). The question of generalised tuning is discussed further in this paper.

As demonstrated by Tolman (2002a), the use of altimeter data to assess the spatial variation in the modelled $H_{s}$ error provides a powerful means of identifying sources of systematic model error. This approach has been used by a number of subsequent studies. Tolman (2003) for example, showed that an overestimation of wave heights in the tropics could be easily traced to missing wave blocking from island chains that were too small to be explicitly resolved by the grid. The motivating study for the present work of Greenslade and Young (2004b) identified a distinct low bias in the AUSWAM model in the Southern Ocean storm tracks, demonstrating local bias features of primary interest to the Australian region. The global validations and tuning of the recent parameterisations of Ardhuin et al. $(2008,2010)$ have relied heavily on the minimisation of spatial bias over the globe as a tuning diagnostic.

While knowledge of the spatial wave field error undoubtedly offers great insight into the deficiencies of modern wave models, in general, many such studies are conducted without sufficient knowledge of the corresponding biases in the forcing winds.

\subsubsection{Sensitivity to the Accuracy of the Forcing Winds}

Global scale wave model tuning relies on accurate wind forcing. As wave models improved, error in the forcing wind fields became the major contributor to the total error budget, making it difficult to assess the effect of model improvements, which in turn acted to slow wave model development. Increasing computational power, improved modelling techniques and increased availability of observations 
has facilitated rapid development of NWP capabilities in recent years, greatly improving the surface wind fields available to wave models. This has had positive effects on the quality of wave forecasts. By re-running the (then) current operational WAM model under ECMWF analysis winds, Janssen (2007), for example, estimated that of the improvements seen in operational wave forecasting capabilities at ECMWF in the 10 years prior to publication, about $25 \%$ could be attributed to inherent improvements in the wave model, the remainder being a direct result of improved wind forcing.

The translation of error in the forcing winds into the wave model is not straightforward. Locally, wind errors will produce errors in newly generated wind sea. These errors will also present as errors in the swell propagating away from the generation region. Hence, the wave error at any given point associated with incorrect wind forcing is a result of the integration of wind error over a window of time and space. The addition of error due to an imperfect wave model makes the attribution of error a non-trivial exercise. On global scales, while it has been shown by many studies that the general quality of the wave field increases with improvements in the wind forcing, attributing spatial variation in systematic error to a given source remains a difficult problem.

There have been several studies dealing with the accuracy of atmospheric predictions from the perspective of the wave modeller (e.g. Komen et al., 1984; Cardone et al., 1995, 1996; Khandekar and Lalbeharry, 1996; Janssen et al., 1997; Tolman, 1998b). In general, these studies have employed two methods: 1) compare both wind and wave model data with all the available measurements and/or 2) cross-compare results obtained using several combinations of atmospheric and wave models. The efficiency of the first method alone has historically been limited by the sparseness and intermittency of the measured data, complicated by the difficulty in making inferences based on local conditions alone. By comparing a number of different models containing different error characteristics, sources of error can be further isolated.

The inability to make simple, local inferences regarding wind/wave error attribution is reduced somewhat by examining single, intense storms events. Under such conditions, where high frequency energy dominates the wave spectrum, error attribution is primarily a local consideration. Perhaps the most well known such study is that of Cardone et al. (1996). Simulations of the so called 'Halloween Storm' of 1991, off the east coast of North America, were produced with com- 
binations of wave models, forced with several different operational forecast wind products. These were then compared to the results of a run performed using a highly accurate, objectively analysed wind field. From comparisons with a number of moored buoys, they conclude that in the then operational nowcast/forecast systems, wind forcing was the dominant source of wave model error.

Similarly, by examining enclosed basins, the influence of propagating error is reduced. Bertotti and Cavaleri (2004), for example, considered the influence of spatial resolution on the underestimation of wind speeds and wave heights in the Mediterranean Sea. In a number of simulations with the WAM model, using ECMWF forcing winds, they found that wind and wave accuracy was strongly dependent on distance from land, with coastal regions generally improving with model resolution. They suggest that the surrounding orography, and its effects on the modelled wind were the major cause of error. Similar conclusion regarding reliability at the coast were found by Ardhuin et al. (2007), examining results from four different meteorological models and three different wave models.

In a more general sense, there have been a number of studies examining the contribution of systematic bias in the forcing winds to that of the modelled waves in the open ocean. Rogers and Wittmann (2002) compared surface winds from the Navy's Operational Global Atmospheric Prediction System (NOGAPS), run at Fleet Numerical Meteorology and Oceanography Center (FNMOC), and the National Centers for Environmental Prediction (NCEP)'s Global Forecasting System (GFS) analyses to QuickSCAT scatterometer data in the northeast Pacific during January 2001 and in the South Pacific during July 2001. They found that both analyses tend to be biased low at high wind speeds, this tendency being relatively slight with the GFS analyses, and stronger in the NOGAPS analyses, particularly in the north-east Pacific comparison. Rogers (2002) made similar direct wind evaluations, but over the globe for 1 January-8 February 2002; these similarly suggested that strong surface wind events in the NOGAPS analyses were biased low. Consequently they concluded that the dominant source of error in predictions of low-frequency wave energy in FNMOC's global models appeared to be due to inaccuracies in the wind forcing. Bias associated with the wave model itself was believed to be only secondary.

This work was repeated, following upgrades to the atmospheric model NOGAPS in the second half of 2002 (Rogers et al., 2005). This led to improvement in the operational global wave model. Following these changes, the authors conclude 
that wind error was no longer the primary cause of total wave model error. These studies, however, focussed on comparisons with a number of buoys around the United States (U.S.) coast, and interpretation on global scales remains difficult using these point based measurements.

In recent years, the high quality and spatial coverage of scatterometer data has become a valuable source of data for the study of marine surface winds (e.g. Kelly, 2004). These data have been extensively used for verification of NWP winds (e.g. Rogers and Wittmann, 2002; Yuan, 2004; Isaksen and Janssen, 2004; Schulz et al., 2007). Recent work examining the spatial variability of the operational ECMWF and NCEP analyses (Chelton and Freilich, 2005) has shown that these two products contain significant spatial structure in the overall bias. As discussed above, a knowledge of the spatial $H_{s}$ bias has greatly enhanced the ability to diagnose and address sources of systematic error in modern wave model source terms. However, while the contribution of bias in the forcing winds is often considered in a general sense, the extent to which a spatial variation in systematic wind speed error contributes to that of the waves has not received a lot of attention.

\subsubsection{Statistical Wind Corrections}

Where systematic errors in the wind field are known a-priori, these can be removed with statistical corrections. Tolman (1998b), for example, conducted a validation of the GFS surface wind speeds prior to the operational implementation of WW3 at NCEP. For the Northern Hemisphere winter of 1994/1995, wind speeds were compared to buoy and ERS-1 altimeter and scatterometer data. Based on this analysis, a wind correction was introduced, prior to tuning the wave model. This correction consisted of a coastal error correction up to $150 \mathrm{~km}$ offshore, a deep-ocean correction beyond $300 \mathrm{~km}$ offshore, and a smooth blending in between. By early 1997 wind speed biases were greatly reduced, resulting in an adjustment to these corrections. Further improvements in the atmospheric model in June 1998 again reduced wind speed biases, resulting in the removal of these corrections in June 1998 (Tolman et al., 2002).

As mentioned above, similar statistical corrections have been applied to wind fields from the Bureau's previous operational atmospheric model GASP prior to forcing the wave model AUSWAM. The low bias in the GASP winds identified 
by Kepert et al. (2005) and Schulz et al. (2007) was removed with a simple linear correction, which was found to have positive effects on the forecast $H_{s}$, when compared to buoy data around the Australian coast (Greenslade et al., 2005).

While such adjustments provide a useful means of removing known biases in the forcing winds, these simple corrections contain a number of limitations. The first is the inability to account for spatial variation in the wind error; winds corrected in this way can be expected to retain significant regional biases. In the case of verifications of the Bureau's GASP marine winds, for example, neither Kepert et al. (2005) or Schulz et al. (2007) included a spatial assessment of error, with a single correction proposed for the entire global domain. Subsequent work has demonstrated that the GASP model showed considerable regional variation in bias, with positive biases in the Tropics, and negative biases in the mid-latitudes (Durrant and Greenslade, Submitted). The corrections applied by Greenslade et al. (2005), while improving the mid-latitudes bias, likely accentuated those in the Tropics, and the observed improvements in wave model results at Australian mid-latitude buoys would be unlikely to be seen everywhere. Tolman (1998b) did consider the spatial variation of error, and partly accounted for this by applying separate corrections around coastlines. However, in the open ocean, the proposed correction simply prioritised bias minimisation in the Northern Hemisphere, in accordance with NCEP's forecasting priorities, actually introducing a slight positive bias in the Southern Hemisphere.

The second limitation, is the need to continually monitor changes in systematic wind biases with alterations and upgrades to the atmospheric model. This point is well illustrated by the development, subsequent reduction and finally removal of statistical corrections at NCEP discussed above. The realities of changed forcing is an issue for any downstream model. Part of the motivation for performing such wind corrections is an acknowledgement that these wind error characteristics are likely to change. Adjustments of statistical wind correction with these changes, are less burdensome than the retuning of the wave model itself. However, maintaining such wind corrections still requires considerable effort. 


\subsubsection{Implications for Operational Forecasting}

Despite the acknowledged impact of wind error on the quality of wave forecasts it is an often ignored facet of current operational wave model tuning. Where the provision of accurate wave estimates is paramount, such as in the context of operational wave forecasting systems, the model is set up, verified and tuned. This tuning is often done based on the forcing as it is, not as it should be.

This is not a criticism. A knowledge of the role of the forcing wind on the accuracy of the wave forecast, while an important research question, is secondary to the provision of accurate wave forecasts under whatever forcing is available. However, where systematic errors in the forcing winds exist, tuning the wave model in isolation in this way is likely to result in the wave model tuning compensating for these errors.

Within a general discussion of the highly tuned state of current wave models Cavaleri (2009) lists the example of the U.K. MetOffice (UKMO) model, used until a few years ago (Golding, 1983; Holt, 1994). They state that this secondgeneration wave model would be expected, on average, to produce an underestimate of the wave field (Cavaleri (2009) offer (Bidlot et al., 2002) in support of this statement, though it is never explicitly stated therein). However, in daily operational applications, this was compensated for by an overestimate of the driving wind speeds derived from the UKMO meteorological model (Bidlot et al., 2002).

This raises an interesting question regarding the tuning of wave models in general. While modern wave model parameterisations are the result of a great many contributers, the source terms primarily in use today are those of Bidlot et al. (2007) and Tolman and Chalikov (1996). Global tuning of these terms has been performed under ECMWF (Bidlot et al., 2007) and NCEP (Tolman, 2002a) wind products respectively. The previously mentioned work of Chelton and Freilich (2005) examined and compared the operational winds from these two institutions, demonstrating not only significant spatial structure in the overall bias, but also significantly different bias characteristics between them. The question then arises, for the purposes of producing accurate wave forecasts using a given set of source terms, are winds that closely match reality the desired forcing product, or those that most closely resemble the winds under which the source terms were developed? 
This point was highlighted in the work of Feng et al. (2006). They performed a number of hindcast studies using WW3 forced by different wind products, including reanalysis products from NCEP and ECMWF, as well as a blended product using NCEP operational winds and QuikSCAT observational data. While they conclude that the wave model accuracy is critically sensitive to wind quality, they found that the overall $H_{s}$ bias, when compared to TOPEX/POSEIDON (TP) altimeter $H_{s}$ data, showed the best results when forced with the NCEP reanalysis product, despite these winds indicating a negative bias when compared to the TP wind data. The $H_{s}$ forecasts produced with the other wind products were all positively biased, with the highest bias from the QuikSCAT blended product. They conclude that this is likely due to the fact that the WW3 model they were using was tuned using winds similar to those of the NCEP reanalysis.

In applications such as operational forecasting, it could be argued that wind errors that compensate for wave model deficiencies are a good thing. However, for the purposes of wave model development, such compensation serves to hinder the identification of sources of systematic wave model error, delaying the address of the causal issue. A knowledge of the wind error, and an understanding of how this manifests in the wave model is hence an important aspect of the further refinement of the source terms.

\subsection{Aim of the Present Study}

This study aims to assess the contribution of systematic error in the forcing surface winds to that of the global modelled wave field. Altimeter and scatterometer observations of $H_{s}$ and $U_{10}$ respectively will be used to determine the spatial structure of the systematic bias of both the modelled winds and waves, providing a powerful platform to assess the relative role of the wind in the total error budget of a modern wave model. Various means of statistically improving the winds are then explored, and the resulting effects on the wave model are analysed.

Specifically, the following questions are addressed.

- To what extent can the large scale systematic biases in the waves be attributed to the forcing winds?

- Do statistical adjustments to remove these wind biases lead to better wave forecasts? 
- What are the residual wave biases when forced with unbiased winds?

\subsection{Models Used in this Study}

The model used in this work is the most recent release of the WW3 model, version 3.14 (Tolman, 2009). The forcing winds come from the Bureau's recently implemented ACCESS system. The winds examined here are from a test configuration of the global model (identified as experiment 00ho). For the period examined, QuikSCAT data has been assimilated in the ACCESS system, and for verification purposes, these data cannot be considered independent at the analysis time. Hence, short term forecast winds from 0-12 hour lead time are used. This ensures high quality winds are considered with minimal phase error, while still maintaining the independence of the verification data.

The original intention was to examine an 8 month period, from July 2008 to February 2009. Unfortunately, due to some data archiving errors in the ACCESS model, winds from the latter half of this period had to be rejected, and only July to October 2008 is used. This has the unfortunate consequence of restricting the period of this study to the Southern Hemisphere winter, limiting the conclusions that can be drawn to this season alone.

\subsection{Structure of Thesis}

Wave model verification carried out in this work relies heavily on altimeter data. Fast Delivery (FD) data from the both the Jason-1 and Envisat missions are used here. Previous work has shown altimeter data typically contain systematic errors (e.g. Cotton and Carter, 1994), although the precise nature of these errors has been found to vary somewhat from mission to mission. Hence, before these data can be used with confidence, their accuracy must be assessed and appropriate systematic corrections developed. Chapter 2 presents a validation of altimeter $H_{s}$ data against in-situ buoy data for each of these data streams. This work has been published in the Journal of Oceanic and Atmospheric Technology (Durrant et al., 2009a).

The ACCESS surface winds used to force the wave model in this study have not been previously verified, and the error characteristics of these winds is un- 
known. In Chapter 3, these winds are compared to QuikSCAT scatterometer observations. This includes an analysis of the spatial structure of the error over the globe. Parts of this work are replicated in a submission to the Australian Meteorological and Oceanographic Journal (Durrant and Greenslade, Submitted), comparing ACCESS winds with those of the Bureau's previous operational model GASP.

Chapter 4 documents both the set up and evaluation of the WW3 wave model against both buoy and altimeter data. With the overall aim of this thesis being to assess the importance of wind error on the accuracy of the wave model forecast, care is taken here to minimise sources of external error such as sea ice, bathymetry, unresolved island blocking etc. A number of runs are performed and verified, broadly assessing the impact of choices of source terms and numerics on the spatial error characteristics when forced with ACCESS winds. Parts of this work, published in slightly different form, appear in the Centre for Australian Weather and Climate Research (CAWCR) Technical Report (Durrant and Greenslade, 2011), assessing WW3 for operational implementation at the Bureau.

Chapter 5 explores a number of means of removing the wind biases identified in the wind verifications conducted in Chapter 3. These include simple homogeneous corrections, as well as spatially varying corrections. The need to monitor wind corrections and/or the considerable effort needed to maintain a tuned wave model in an operational environment subject to evolving atmospheric models has been discussed above. With this in mind, a automatic, self learning correction method is proposed, applicable to operational forecast winds. This work was performed with the intention of developing an operational system applicable to the Bureau's forecasting environment.

The sensitivity of the wave model to the now known biases in the forcing winds is then discussed in Chapter 6. The impact of the wind corrections determined in Chapter 5 on the modelled wave field is examined, and the extent to which large scale systematic error over the globe can be attributed to the spatial structure of systematic error in the wind is analysed. It is in this chapter that the questions raised above are addressed.

Finally, a summary and conclusions are presented in Chapter 7. A list of abbreviations used throughout this thesis is given in Appendix A. 


\section{Chapter 2}

\section{Validation of Jason-1 and Envisat Significant Wave Height}

Contents

2.1 Introduction . . . . . . . . . . . . 15

2.2 Previous Work . . . . . . . . . . . 16

2.2 .1 Jason-1 . . . . . . . . . . . . . . . . . . . . . . . . 18

2.2 .2 Envisat . . . . . . . . . . . . . . . . . . 19

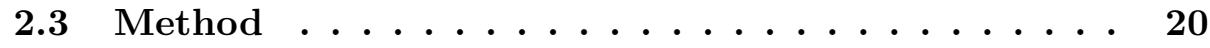

2.3 .1 Data . . . . . . . . . . . . . 20

2.3 .2 Method .................... 21

2.4 Results .................... 23

2.4.1 MEDS and NDBC buoy network differences . . . . . . 23

2.4.2 Validation Results . . . . . . . . . . . . . 27

2.4 .3 Wave State Influence . . . . . . . . . . . . . . 30

2.5 Summary $\ldots \ldots \ldots \ldots \ldots \ldots \ldots \ldots$

\section{$2.1 \quad$ Introduction}

The wave model validation carried out in this thesis relies heavily on altimeter data. Jason-1 and Envisat FD data are used here. Before they can be used with confidence, their quality must be assessed, and if necessary, appropriate 
corrections determined. This is the subject of this chapter. Data from both altimeters are validated against in situ buoy data. A number of correction schemes are then investigated with the aim of minimising the overall root-mean-squaredifference (RMSD). Section 2.2 examines the previous validation work for each altimeter. Section 2.3 describes the data and method used, followed by a discussion of the results in Section 2.4 and finally Section 2.5 states some conclusions.

\subsection{Previous Work}

Seven satellite altimeters have been deployed since 1985, providing the first long term global observation of sea level, wind speed and wave height. Steady improvement has been made with each subsequent satellite, though characteristic biases have tended to vary somewhat from one to the next. Careful calibration of altimeter estimates against in situ measurements is thus important for accurate global estimation of $H_{s}$.

Altimeter validation studies, in general, attempt to obtain a set of co-located altimeter and buoy observations and find an appropriate adjustment for the altimeter which results in the best fit with the in situ buoy data. Comparisons between buoy and altimeter derived $H_{s}$ data are complicated by the fact that each is measuring different aspects of the temporally and spatially varying wave field, and hence may differ, even in the case that both instruments are making perfectly accurate estimates (Monaldo, 1988).

Differences can be divided into three categories, temporal proximity, spatial proximity and sampling variability associated with time and space averaging. Due to the frequency of satellite passes, altimeter-buoy measurements can not always be made simultaneously. Usually, a temporal window of acceptability is established; a maximum time difference for measurements to be considered a comparable altimeter-buoy pair. Similarly, an acceptable spatial separation between the altimeter track and the buoy location must be established. Sampling variability occurs due to the fact that altimeter measurements are essentially an instantaneous spatial average of $H_{s}$ over the altimeter footprint area, which increases from a diameter of about $3 \mathrm{~km}$ for small wave conditions to about $10 \mathrm{~km}$ for large wave conditions, while buoy measurements are time-averaged measurements of $H_{s}$ at a point location. 
Based on assessments of the spatial and temporal variation of the wave field, Monaldo (1988) proposes co-location criteria of observations occurring within 50 $\mathrm{km}$ and 30 minutes of one another. These criterion have been widely adopted since, and now provide the standard for this type of work.

The two altimeters examined in this work are onboard the Jason-1 and Envisat satellites. Launched in December 2001, Jason-1 is a jointly operated project between the French and U.S. space agencies, the Centre National d'Etudes Spatiales (CNES) and the National Aeronautics and Space Administration (NASA) respectively. It follows on from the successful TOPEX/POSEIDON (TP) mission, sharing the same orbital parameters and following in the same ground tracks as its predecessor. It flies in a non-sun-synchronous orbit at an altitude of 1336 $\mathrm{km}$ and an inclination angle of $66^{\circ}$. It carries a Poseiden- 2 altimeter, derived from the experimental Poseiden-1 instrument carried on the TP mission. Detailed descriptions of the mission and the Poseiden-2 altimeter can be found in Menard et al. (2003) and Carayon et al. (2003) respectively. Envisat, launched March 2002, is operated by the European Space Agency (ESA). This mission follows on from European Remote Sensing Satellite - 1 (ERS-1) and European Remote Sensing Satellite - 2 (ERS-2), carrying the so called RA-2 altimeter derived from these earlier missions. Envisat flies in a sun-synchronous orbit at an altitude of $800 \mathrm{~km}$, and inclination angle of $98^{\circ}$, this angle allowing Envisat to take observations closer to the poles than Jason-1. More detail can be found in Resti et al. (1999). Typical daily coverage is shown in Figure 2.1, indicating ground tracks for both satellites during a $24 \mathrm{~h}$ period.

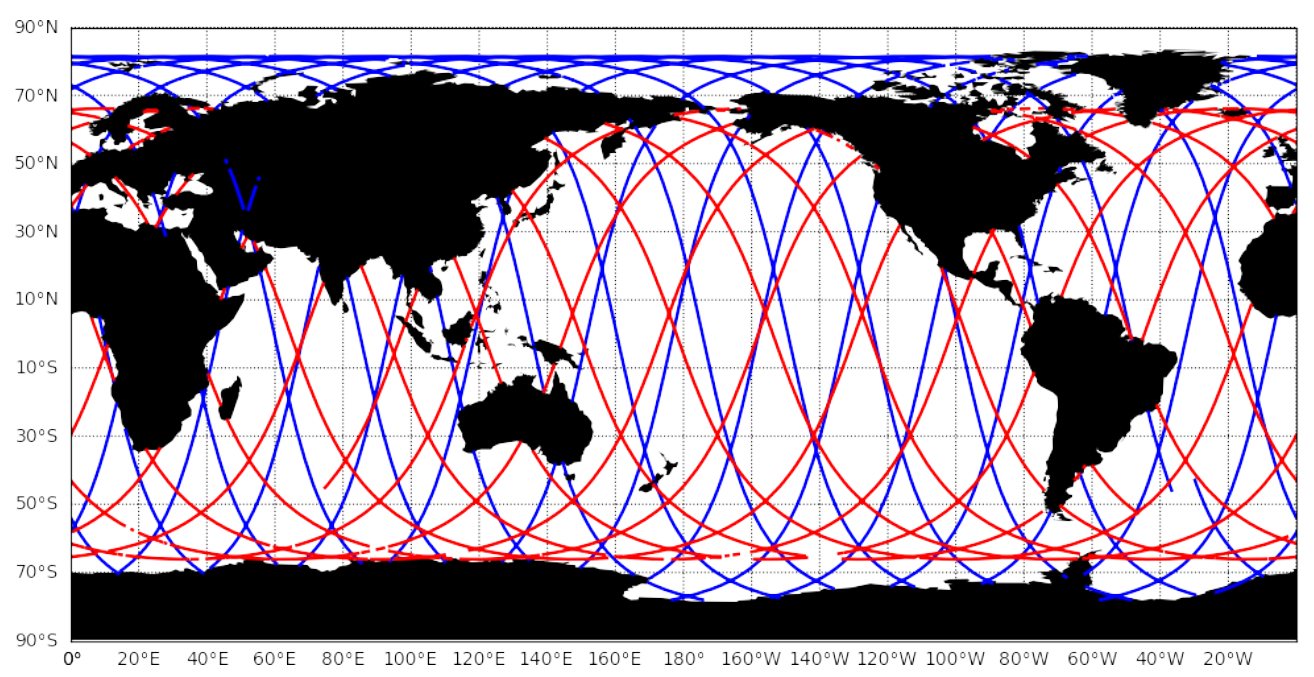

Figure 2.1: Altimeter ground tracks from one day for Jason-1 (red) and Envisat (blue). 
Multiple data streams are available from these satellites. These can be divided into operational, or so called FD streams, and higher accuracy, more detailed off-line streams. The former is used primarily for operational data assimilation and is available within 2-3 hours of observation, while the latter is typically available several days later. Radar altimeters are active microwave sensors which infer $H_{s}$ directly from the shape of the radar pulse, or waveform, returning to the nadir-looking altimeter. FD products differ primarily from their off-line counterparts by the fact that the former consists of reports derived from onboard processing of these waveforms, while ground based processing is performed for the off-line stream. In this work, the FD stream is used, referred to as the Operational Sensor Data Record (OSDR) in the case of Jason-1, and the Near Real Time (NRT) stream for Envisat.

Although both Jason-1 and Envisat have been in orbit for a number of years, there is little published work on the validation of $H_{s}$ data from these satellites, especially the FD products. The following summarises the published validation studies for each altimeter.

\subsubsection{Jason-1}

Though there is little validation work available for FD Jason-1 data, there has been some work examining the off-line product. For these studies to be used as a context for the work presented here, it must be known how the FD and off-line products compare. Desai and Vincent (2003) investigated precisely this, comparing 40 days of data from each stream. Inclusion of sea-state bias calculations based on, among other things, wind and wave conditions in the off-line product, result in significant differences between these streams for sea surface height data. For $H_{s}$, they conclude that while the FD product does show slightly less scatter, only small systematic differences exist between the FD and off-line products, which appear to be well described by the linear relationship.

$$
H_{s}^{F D}=1.053 \times H_{s}^{O L}+0.011 \mathrm{~m}
$$

where $H_{s}^{F D}$ is the FD $H_{s}$ and $H_{s}^{O L}$ is the off-line $H_{s}$. Assuming the validity of this result, some work validating off-line $H_{s}$ data with in situ buoy data is now relevant. One of the earliest studies was performed by Ray and Beckley (2003) using 6 months of off-line data from February to August 2002. They found a $7 \mathrm{~cm}$ 
negative bias, suggesting a correction of (with $95 \%$ confidence levels indicated):

$$
H_{s}^{a d j}=1.100( \pm 0.015) \times H_{s}^{O L}-0.104( \pm 0.024) \mathrm{m}
$$

where $H_{s}^{a d j}$ is the corrected $H_{s}$. This study is of limited reliability however, as conclusions are based on only 368 co-locations.

In an attempt to provide an homogeneous data set spanning several satellites, Queffeulou (2004) validates $H_{s}$ data from six altimeters, using cross-altimeter and buoy comparisons. Using $50 \mathrm{~km}$ along track averages and a co-location separation criteria of 30 minutes, his study covers the period January 2002 to September 2003 and resulted in 2853 co-locations for Jason-1. Overall, he found a $5 \mathrm{~cm}$ negative bias in the altimeter data relative to the buoy data, finding the following linear relation:

$$
H_{s}^{a d j}=1.0072 \times H_{s}^{O L}+0.0392 \mathrm{~m}
$$

Substituting equation 2.1 into equations 2.2 and 2.3 gives the following corrections to FD data respectively:

$$
\begin{aligned}
& H_{s}^{a d j}=1.045 \times H_{s}^{F D}-0.115 \mathrm{~m} \\
& H_{s}^{a d j}=0.957 \times H_{s}^{F D}+0.029 \mathrm{~m}
\end{aligned}
$$

It must be stressed that Equations 2.4 and 2.5 are inferred from relationships found between off-line data and buoy data, and off-line data and FD data found in different studies, and hence cannot be interpreted as quality validations. Despite this, they suggest that little correction to this data is required. This is consistent with work done at Meteo-France (Lefevre, personal communication, 2006) where biases have been shown to be small, and FD data is not corrected prior to assimilation. The ECMWF applies a reduction of $4 \%$, based on comparisons with buoy and model data (Abdalla et al., 2005).

\subsubsection{Envisat}

Queffeulou (2004) performed a validation of Envisat $H_{s}$ data, using 30 minutes 
temporal and $50 \mathrm{~km}$ spatial windows, from April 2003 to February 2004, yielding 1280 co-locations. He proposes a correction of:

$$
H_{s}^{a d j}=1.0327 \times H_{s}^{O L}-0.1830
$$

However, this is using the off-line product, and while the results of Desai and Vincent (2003) mentioned above regarding comparisons between FD and off-line data for Jason-1 are encouraging, the relationship between these two streams for Envisat remains unknown.

Abdalla (2006) has carried out a thorough investigation on Envisat FD windwave performance based on data from July 2002 to October 2003. Although no correction is proposed, they estimate that the altimeter overestimates $H_{s}$ by about $9 \mathrm{~cm}$ relative to buoy data. As a result of this work, ECMWF applies the same correction to Envisat as is applied to the Jason-1 data, i.e. a bulk reduction of $4 \%$ (Bidlot, personal communication, 2006)

Overall, there is a dearth of work validating the FD data for both these altimeters. Those that do exist, have often been obtained through informal validations in an operational environment. As such, the need exists for a documented validation of these data streams.

\subsection{Method}

\subsubsection{Data}

Buoy data are generally assumed to be of high quality, and have been used in numerous studies for validation of model data (e.g. Janssen et al., 1997; Caires and Sterl, 2003; Caires et al., 2004) and altimeter data (e.g. Tolman, 2002a; Queffeulou, 2004; Faugere et al., 2006). Buoy data used in this work are obtained from two buoy networks, the U.S. operated National Data Buoy Center (NDBC) network and the Marine Environmental Data Service (MEDS) network operated by Environment Canada. Buoy data from the Australian network was considered, but buoy locations were too close to the coast, or reporting frequency too low to be of value. In the case of both selected networks, rigorous quality control is undertaken by these institutions, with bad data being either flagged, or removed completely from the data set. These networks are dynamic, with new buoys 
regularly being added, and others being removed.

Altimeter data was obtained from several sources. Near real time data from both Jason-1 and Envisat are received at the Bureau through the Global Telecommunication System (GTS) and archived. Envisat data was extracted from this archive for the period from September 2004 through to April 2006, with earlier data from April 2003 being obtained from CNES. Similarly, Jason-1 data was extracted for the period from September 2004 through to the end of March 2006 with data from January 2002 obtained from Meteo-France. For each altimeter, a month of overlapping data was analysed to ensure consistency and accurate decoding from the two sources. This gave data sets covering a period of three years for Envisat, and four and a half years for Jason-1.

\subsubsection{Method}

Though a $50 \mathrm{~km}$ spatial proximity criterion, or thereabouts, is widely used for altimeter validation studies, it is often replaced by a simple latitude-longitude proximity (Janssen et al., 1997; Caires and Sterl, 2003; Greenslade and Young, 2004a, for example). The obvious shortcoming of this method is that zonal proximity varies with latitude. It could be argued, as is evident by its widespread use, that this will not greatly affect validation conclusions. However, using this technique, it was found that the latitudinal dependence of the error characteristics varied for each altimeter, a result arising from their differing inclination angles. Further details of these differences can be found in Appendix B. For the remainder of this work a strict $50 \mathrm{~km}$ great arc proximity requirement was adopted.

At each buoy, consecutive altimeter observations crossing an area within a $50 \mathrm{~km}$ radius of the buoy location were grouped together. Passes took less than a minute to traverse this area, with each pass typically resulting in 12-16 individual observations that can be assumed to be simultaneous. These data were quality controlled by removing individual observations greater than \pm two standard deviations away from the mean for that pass. Hourly buoy data were then linearly interpolated to the altimeter overpass time, with the additional criterion that there be at least one buoy observation within one hour before and one hour after the time of the altimeter overpass. This interpolated buoy data and the mean of the quality controlled altimeter observations made up a single 
co-location.

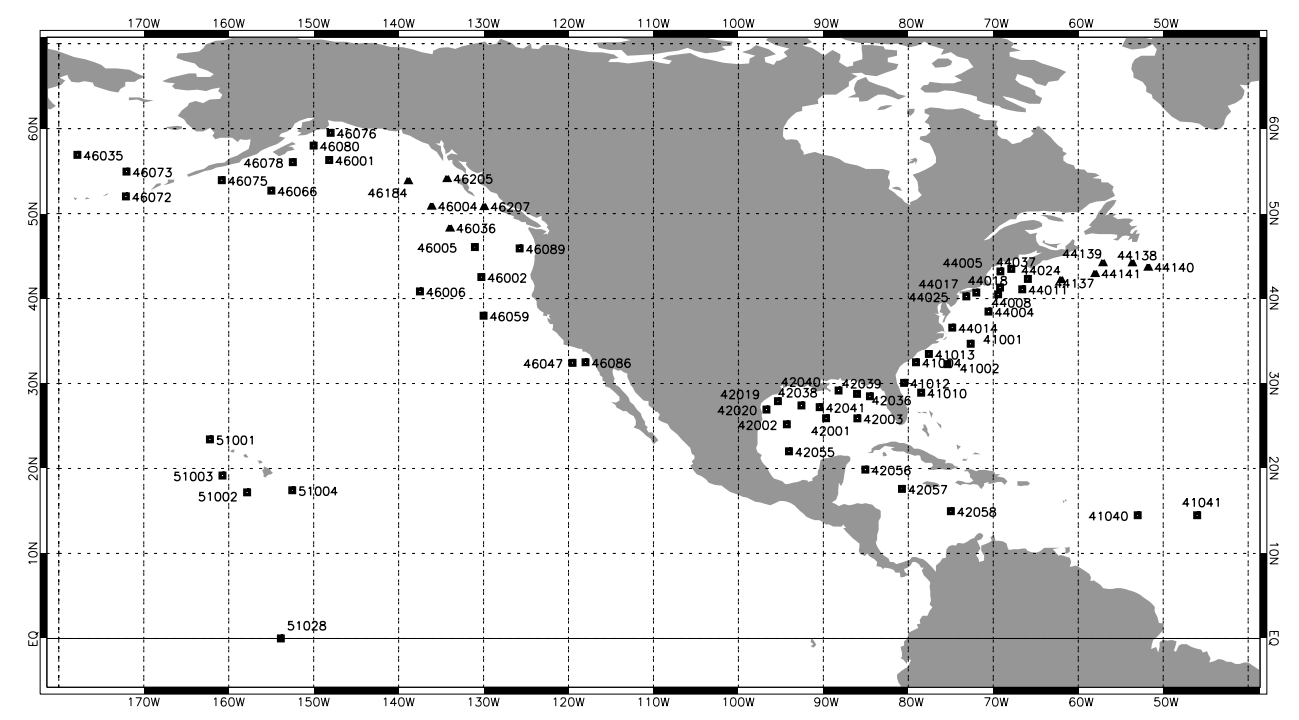

Figure 2.2: Buoys used in the altimeter validation. NDBC are marked with squares, MEDS buoys are marked with triangles

During the early altimeter missions, errors associated with buoy data were small relative to those of the altimeter. As such, buoy data were generally regarded as 'truth' and hence an appropriate procedure was to regress the altimeter data onto the buoy data, i.e. perform an Ordinary Least Squares (OLS) regression with the altimeter data as the independent variable and the buoy data as the dependent variable. As the relative error of the altimeter has reduced, more sophisticated regression techniques have been applied to the problem which account for errors in both variables (e.g. orthogonal regression, errors-in-variables, principal component regression). These techniques generally require a knowledge of the error variances of the two data sets. Caires and Sterl (2003) conclude that error variances for altimeter and buoy data are approximately equal. This has since been adopted by many others (e.g. Queffeulou, 2003; Ray and Beckley, 2003; Soukissian and Kechris, 2007), and is assumed here. The derivation of appropriate corrections are calculated using Total Least Squares (TLS) methods (also known as errors-in-variables). The merits of this method are discussed in many papers (e.g. Isobe et al., 1990; Soukissian and Kechris, 2007).

The statistics used here are the bias, RMSD, Scatter Index (SI) and linear correlation coefficient $(\mathrm{R})$, defined as follows: 


$$
\begin{gathered}
\text { Bias }=\frac{1}{N} \sum_{i=1}^{N}\left(A_{i}-B_{i}\right) \\
R M S D=\sqrt{\frac{1}{N} \sum_{i=1}^{N}\left(A_{i}-B_{i}\right)^{2}} \\
S I=\frac{\sqrt{\frac{1}{N} \sum_{i=1}^{N}\left(\left(A_{i}-\bar{A}\right)-\left(B_{i}-\bar{B}\right)\right)^{2}}}{\mathrm{R}=\frac{\sum_{i=1}^{N}\left(A_{i}-\bar{A}\right)\left(B_{i}-\bar{B}\right)}{\sqrt{\sum_{i=1}^{N}\left(A_{i}-\bar{A}\right)^{2}\left(B_{i}-\bar{B}\right)^{2}}}}
\end{gathered}
$$

where $A_{i}$ is the altimeter $H_{s}, B_{i}$ is buoy $H_{s}, N$ is the number of co-locations and an overbar represents the mean value.

\subsection{Results}

\subsubsection{MEDS and NDBC buoy network differences}

The initial collation of the co-located data revealed an apparent systematic difference between $H_{s}$ estimates from the MEDS and NDBC buoy networks. Figure 2.3 shows scatter plots of co-locations for both altimeters for both NDBC and MEDS buoys separately. In the case of both satellites, it is clear that the MEDS buoys are underestimating buoy $H_{s}$ measurements relative to the altimeter. Jason-1 shows little bias relative to NDBC buoys, but a $28 \mathrm{~cm}$ bias relative to the MEDS buoys. Similarly for Envisat, a small bias of $3 \mathrm{~cm}$ relative to NDBC buoys compares to $28 \mathrm{~cm}$ for the MEDS co-locations. This systematic underestimation appears to be linearly related to $H_{s}$. The SI indicates a similar amount of noise for comparisons with both altimeters, suggesting a comparable level of noise for the buoy networks relative to each other.

To quantify the difference between buoy networks, altimeter $H_{s}$ estimates were taken as a reference against which buoy networks could be compared. Reference to the altimeter $H_{s}$ as truth is deliberately avoided here. As discussed in Section 2.3, neither buoy nor altimeter measurements can be regarded as truth and both must be assumed to contain errors. However, under the assumption that the altimeter provides self-consistent, and repeatable measurements, it is 

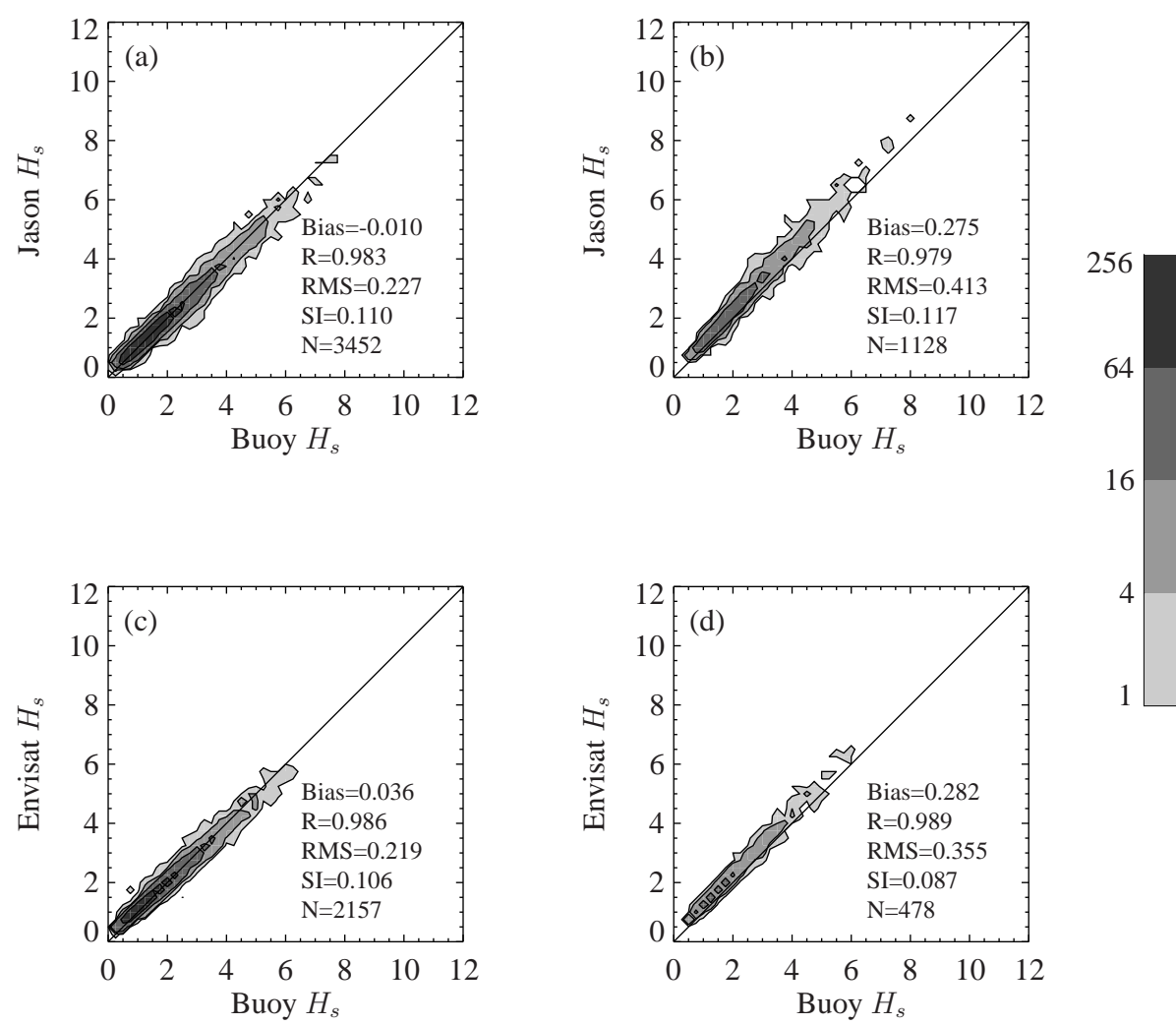

4

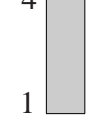

Figure 2.3: Scatter plots of co-located $H_{s}$ observations for Jason-1 and Envisat for both the NDBC and MEDS buoy networks separately. Panels on the top (a and b) show Jason-1 data while those on the bottom (c and d) show Envisat data. Panels on the left (a and c) show co-locations with NDBC buoys only, those on the right ( $b$ and $d$ ) show co-locations with MEDS buoys only. The number of co-locations in each $0.5 \mathrm{~m}$ bin have have been contoured 
valid to employ it as a common reference.

For each network, an OLS regression is performed with buoy data as the independent variable and the altimeter data as the dependent variable. This provides altimeter $H_{s}$ as a linear function of both NDBC and MEDS buoys, given by equations 2.11 and 2.12 respectively for Jason-1 and 2.13 and 2.14 for Envisat.

$$
\begin{aligned}
& H_{s}^{J A S}=0.947 \times H_{s}^{N D B C}+0.099 \mathrm{~m} \\
& H_{s}^{J A S}=1.050 \times H_{s}^{M E D S}-0.143 \mathrm{~m} \\
& H_{s}^{E N V}=0.909 \times H_{s}^{N D B C}-0.222 \mathrm{~m} \\
& H_{s}^{E N V}=1.023 \times H_{s}^{M E D S}-0.225 \mathrm{~m}
\end{aligned}
$$

These expressions are then equated to give a relationship between the two buoy networks. It is stressed here that these expressions do not present proposed corrections. Corrections are derived in the next Section using TLS regressions, rather than the OLS regressions used here. Equating equations 2.11 and 2.12, and 2.13 and 2.14 yields the following linear relationships between MEDS and NDBC buoy $H_{s}$ estimates as determined from Jason-1 (2.15) and Envisat (2.16) as a reference.

$$
\begin{gathered}
H_{s}^{M E D S} \approx 0.90 \times H_{s}^{N D B C}-0.054 \mathrm{~m} \\
H_{s}^{M E D S} \approx 0.889 \times H_{s}^{N D B C}-0.003 \mathrm{~m}
\end{gathered}
$$

This suggests that MEDS buoys are underestimating $H_{s}$ relative to NDBC buoys by about $10 \%$. To test the significance of this difference, a set of normalised differences was calculated using co-locations with each buoy network. A student t-test was then applied to these two data sets to determine whether they had significantly different means. Results for each altimeter separately showed these means to be different at the $99 \%$ significance level in both cases.

This presents a significant point of note, which, to the author's knowledge, does not appear in the peer reviewed literature. It has however, been noted 
previously, with several conference proceedings indicating similar findings. Most recently, in examining TP, Jason-1 and Envisat $H_{s}$, Queffeulou (2006) showed that the validation results are different according to the buoy network, and in the same way for the three altimeters. For instance, the TP - buoy mean bias is $-0.01 \mathrm{~m}$ for the NDBC network and $0.19 \mathrm{~m}$ for the MEDS network, with similar results reported for the other satellites. Examining two years of altimeter data, Cotton et al. (2004) also identify significant differences in validation results for Envisat and ERS-2 in reference to different buoy networks, with results again showing a greater altimeter bias when compared to MEDS buoys than for that of NDBC buoys. Similar results are also noted in the work of Challenor and Cotton (2001). They present altimeter verification results for all altimeters since Geosat, against several buoy networks. They show that the Japanese buoys measured $H_{s}$ highest $(+6 \%$ cf NDBC), then the UK buoy network $(+4 \%)$, then NDBC (as the reference), with the Canadian network buoys measuring the lowest $(-5 \%)$. However, it is noted that the errors in these analyses were quite high, because at the time, the $H_{s}$ data supplied by the UK Met Office and Japan Meteorological Agency were only provided to the nearest $0.5 \mathrm{~m}$. The size of the buoy platform was investigated as a possible cause of the discrepancy, but no significant dependency was identified. They conclude that the differences are likely due to different reporting standards and quality control.

The source of this discrepancy remains unclear. Initial investigations suggest that it is not environmental. Examining each buoy individually reveals consistent biases amongst buoys in the same network, regardless of their position. Buoy size is also unlikely to be the cause. Again, the consistency within each network, despite each being made up of buoys of various makes and sizes, suggest this is not a significant contributor. Though no evidence is presented here, it is suggested that differences in data processing are likely the primary cause.

Given the prevalence of the use of these buoys, often together as a presumed consistent data set, a discrepancy of this magnitude warrants the attention, not only of the data providers, but also the community of users. Subsequent to the identification and publication of this issue in Durrant et al. (2009a), further work has been carried out with Jean Bidlot, from ECMWF. This work, exploring discrepancies amongst a number of buoy networks, was presented at the JCOMM Technical Workshop on Wave Measurements from Buoys (Bidlot et al., 2008). This, in part, provided the motivation for the formation of the JCOMM Pilot 
Project on Wave measurement Evaluation and Test $(\mathrm{WET})^{1}$. The full details of the group's objectives can be found on the website, or in the community white paper Swail et al. (2010).

\subsubsection{Validation Results}

Based on the discussion above, in order to ensure a consistent reference data set, only NDBC buoys were used for altimeter validation. This choice was motivated by the fact that the NDBC data set is much larger than the MEDS data set. NDBC data has also been more extensively used for this type of work, thereby providing the logical choice in the interests of providing a consistent data set across several satellites.

Using only NDBC buoys yielded 3452 co-locations for Jason-1, and 2157 for Envisat. These co-located data points are shown in Figure 2.4. Table 2.1 provides the proposed corrections and the statistics for both raw and corrected data.

Table 2.1: Validation statistics for raw and linearly corrected data based on NDBC buoy comparisons for both Jason-1 and Envisat. For linearly corrected data, regression coefficients are given based on the relationship $H_{s}^{\text {corrected }}=$ $\alpha H_{s}^{r a w}+\beta$.

\begin{tabular}{lccccc}
\hline Correction & Bias $(\mathrm{m})$ & SI & $\operatorname{RMSD}(\mathrm{m})$ & $\alpha$ & $\beta$ \\
\hline Jason-1 & & & & & \\
Raw & -0.010 & 0.110 & 0.227 & & \\
Linear & 0.002 & 0.110 & 0.227 & $1.041 \pm 0.009$ & $-0.076 \pm 0.001$ \\
Envisat & & & & & \\
Raw & 0.036 & 0.106 & 0.219 & & \\
Linear & 0.004 & 0.100 & 0.203 & $1.093 \pm 0.013$ & $-0.233 \pm 0.001$ \\
\hline
\end{tabular}

The uncorrected Jason-1 data shows a negative bias of only $1 \mathrm{~cm}$ and an RMSD of $23 \mathrm{~cm}$. The correction found with the TLS regressions results in no improvements to the RMSD error, suggesting that Jason-1 does not require correction. As discussed in Section 2.2, results inferred from Ray and Beckley (2003) and Queffeulou (2004) found small negative biases, while the findings of Abdalla (2006) suggest a small positive bias. Overall, all these studies propose minor corrections, and are consistent with the findings here. These perspectives are

\footnotetext{
${ }^{1}$ http: //www $\cdot$ jcomm . info/index $\cdot$ php?option=com_content\&task=view\&id=90
} 

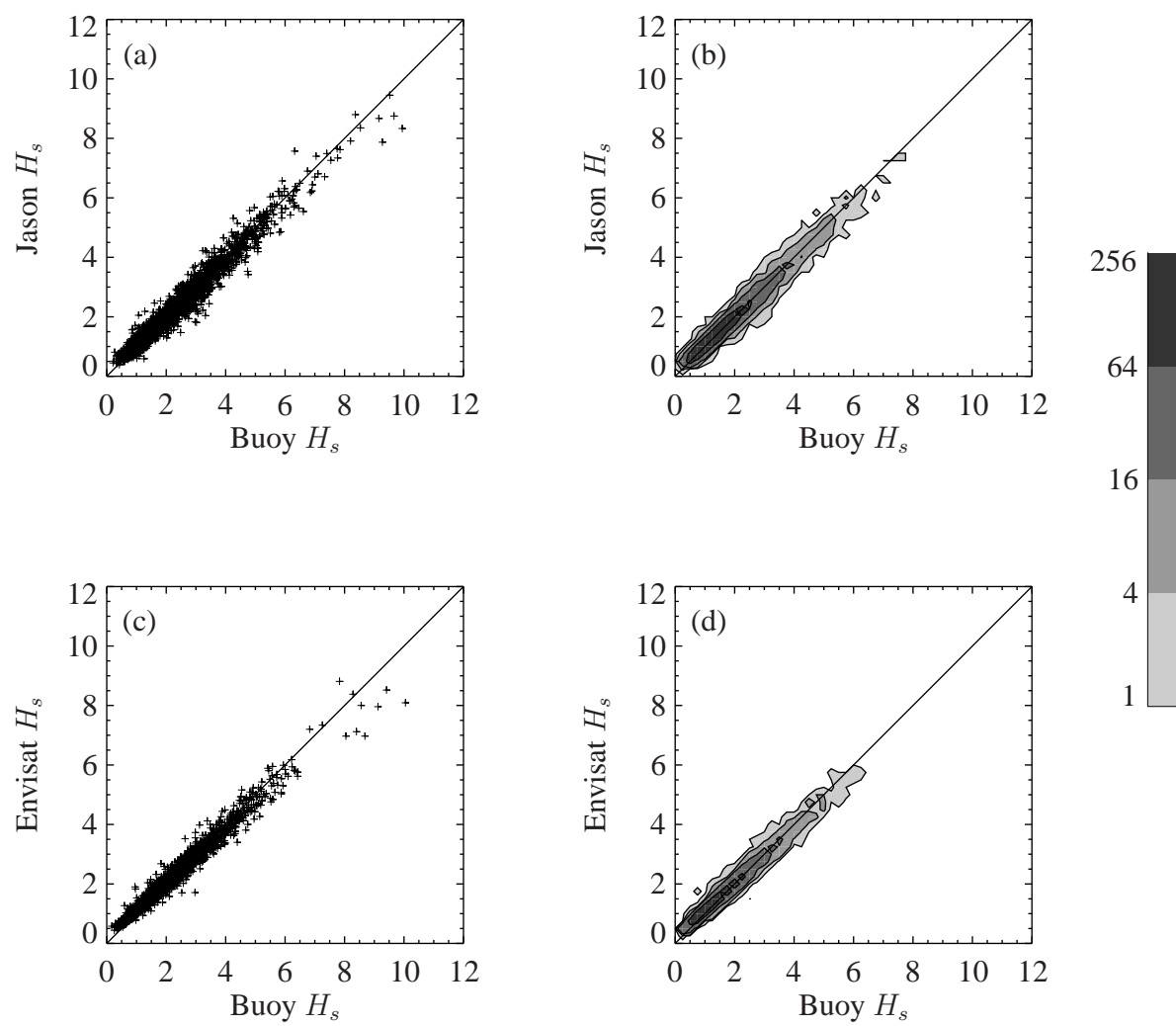

4

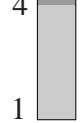

Figure 2.4: (a) Co-located Jason-1 and buoy data and (b) the same data with the number of co-locations in each $0.5 \mathrm{~m}$ bin contoured. (c) and (d) show the same for Envisat data 
also in line with those of Meteo-France, who currently don't apply a correction to Jason-1 FD data prior to assimilation.

For Envisat a small overall bias of $3.6 \mathrm{~cm}$ is present in the uncorrected data, with the altimeter overestimating $H_{s}$. It is apparent from Figure 2.4 that Envisat is overestimating low $H_{s}$ and underestimating high $H_{s}$. Linear correction produces more improvement than seen for Jason-1, decreasing RMSD by 7\%, and the SI by $6 \%$.

In terms of the need for bias corrections, Jason-1 seems to out-perform Envisat. However, Jason-1 is the noisier of the two, with the RMSD being slightly higher than Envisat, despite its biases. Once Envisat is corrected, its RMSD is $0.203 \mathrm{~m}$ compared to $0.227 \mathrm{~m}$ for Jason-1. This relative noise between the two altimeters has also been noted by Abdalla et al. (2005). Similarly, several previous studies have noted an increased level of noise for Jason-1 relative to its predecessor TP (e.g. Cotton et al., 2001; Ray and Beckley, 2003; Queffeulou, 2006). This suggests that the Jason-1 instrument, rather than differences in the data processing, is the cause of this difference.

Envisat appears to have shown significant improvements over its predecessor ERS-2, which suffered from systematic errors at low $H_{s}$ (Greenslade and Young, 2004a). It is worth noting however, that once a branched linear correction was applied, the RMSD reported by Greenslade and Young (2004a) for ERS-2 was similar to that found here for the Envisat corrected data. This suggests that while the systematic biases are still being reduced with each altimeter mission, random error, or noise, is levelling out. This also suggests that a large proportion of the RMSD seen in these results is from the sampling issues discussed in Section 2.2. The previously mentioned work of Monaldo (1988), upon which much of the validity of studies of this nature are predicated, states an expected buoy altimeter RMSD of $0.4 \mathrm{~m}$ assuming perfect measurements from both instruments, due to these sampling issues alone. The fact that results are now better than these theoretical limits suggests that this estimate is in need of revision.

The apparent difference between the variance for each altimeter suggests that the assumption of equal error variances between buoy and altimeter, used in the TLS regressions, is perhaps a generous one. The use of a third independent data set, model data for example, could be used to overcome the need for such an $a$ priori assumption. So called triple co-location techniques, first used by Stoffelen (1998) in application to marine winds, and later by Caires and Sterl (2003) and 
Janssen (2007) for the validation of waves, employ an iterative approach whereby a relative error between the three data sets is assumed, then new estimates are calculated. This is then repeated based on these new estimates until stable values are reached. This is a powerful technique in the presence of a third data set. As the corrections found here are small, the assumption of equal error variance is unlikely to have a significant impact on results.

\subsubsection{Wave State Influence}

Two main classes of surface gravity waves exist in the ocean, namely wind wave (or wind sea) and swell. The former refers to young waves under growth, or in equilibrium with local wind, while the latter is defined as waves generated elsewhere that have propagated some distance. This Section examines whether altimeter $H_{s}$ estimates are influenced by the dominance of either wind sea or swell.

The idea that the wave state may have an influence on the accuracy of altimeter derived $H_{s}$ was examined by Janssen (2000) in relation to ERS-2 FD data. He argues that the 'peakiness' of the waves will affect the retrieval of altimeter wave heights, with the assumption of a Gaussian distribution of sea surface height being invalid for steep wave conditions. It was shown that by adjusting the ERS-2 $H_{s}$ according to the Phillips parameter, $\alpha_{p}$, better agreement could be found between altimeter and buoy $H_{s}$ observations. $\alpha_{p}$ is obtained from wave spectra by parametrising the spectrum as:

$$
F(k)=\frac{1}{2} \alpha_{p} k^{-3}
$$

where $k$ is the wave-number. This is speculative however, and the need for further investigation is noted. Challenor and Cotton (2001) also examined this idea by comparing the normalised altimeter-buoy error in $H_{s}$ against wave age. In that study, no relationship was found.

A similar approach is taken here, examining the direct effects of wave steepness. Specifically, Significant Wave Steepness $\left(S_{s}\right)$ (Tucker and Pitt, 2001) is used, defined in terms of the integral wave characteristics which are reported by the buoys.

$$
S_{s}=\frac{H_{s}}{L_{p}}=\frac{2 \pi H_{s}}{g T_{p}^{2}}
$$


where $L_{p}$ and $T_{p}$ are wave length and period respectively corresponding to the spectral peak frequency. This is also referred to as significant slope by Huang et al. (1981).

It is expected that the effect of wave state on altimeter $H_{s}$ retrievals would apply similarly to both altimeters. Hence, the data sets have been combined here in order to increase the total number of observations and increase confidence in the results. Unless otherwise stated, altimeter data have been linearly corrected, with the corrections given in Table 2.1 .

Figure 2.5 shows the mean bias (altimeter minus buoy) plotted against the wave steepness. The size of the ellipses gives the number of co-locations in each bin. This shows a significant decreasing bias with increasing steepness, adding weight to the findings of Janssen (2000).

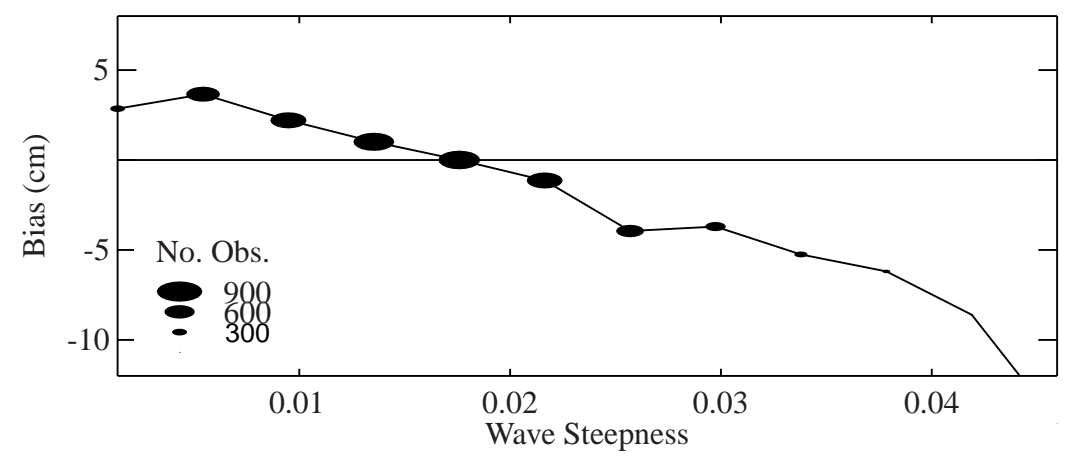

Figure 2.5: Altimeter bias as a function of wave steepness. The size of the ellipses gives the number of co-locations in each bin

However, in examining this plot alone, some care must be taken in the attribution of the cause of these bias characteristics. Figure 2.6 shows mean $H_{s}$ against wave steepness. It is apparent from this that larger waves tend to be steeper than smaller ones. As discussed in Section 2.2, altimeters tend to contain wave height dependent biases that are easily removed with linear corrections. Hence, it is possible that biases associated with wave height could be responsible, at least in part, for these wave steepness dependent biases. In addition, use of $H_{s}$ corrected data here could be masking some of the wave steepness dependent bias.

Figure 2.7 shows altimeter bias as a function of (a) $H_{s}$ and (b) wave steepness for both corrected and uncorrected altimeter data. Focusing first on the $H_{s}$ case, in the uncorrected data, a positive bias is seen for low wave heights and a negative 


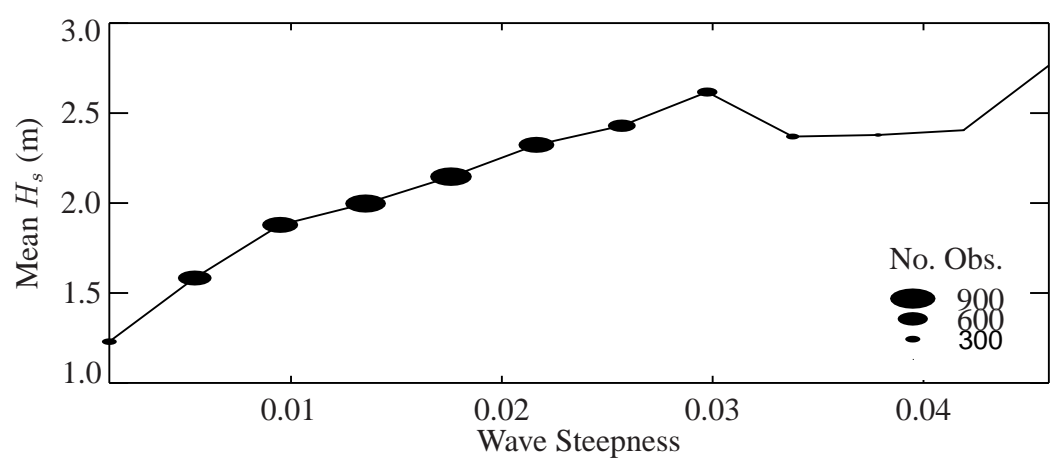

Figure 2.6: Mean $H_{s}$ as a function of wave steepness. The size of the ellipses gives the number of co-locations in each bin

bias for high, as discussed in Section 2.4. This bias is, not surprisingly, almost eliminated by the $H_{s}$ dependent correction. A qualitative assessment of how much of this bias is due to wave steepness can be gained by looking at how this correction affects the bias relative to wave steepness. Examining the second plot, for the uncorrected data, a large positive bias is seen for relatively smooth waves, while steep waves yield a negative bias, a result which could be consistent with both trends having the same origins. However, correcting the data according to $H_{s}$ does not eliminate the wave steepness bias. While there is a reduction in the positive bias in the case of relatively smooth waves, little difference is seen for steeper waves, with a decreasing bias with increasing wave steepness remaining clearly evident. This suggests that altimeter $H_{s}$ retrievals suffer from systematic biases associated with both wave height and wave steepness.

Partial correlations (Wilks, 1995) provide a means of isolating these effects. Table 2.2 shows the Pearson correlation coefficients for the altimeter $H_{s}$ bias against $H_{s}$ and wave steepness, as well as the partial correlations of each variable with the effects of the other removed. $H_{s}$ dependent bias is stronger than that apparent from wave steepness, with correlations of -0.36 and -0.21 respectively. Removing the effects of wave steepness has little effect on the $H_{s}$ correlation. Removing the effects of $H_{s}$ does reduce the steepness correlation somewhat, though it remains significant. This suggests that both effects apply.

There are obvious limitations to using mean wave characteristics to define wave steepness. This analysis would certainly benefit from the use of buoy data which contains spectral information, though this is left here for future work. Given this apparent wave steepness bias dependence, the opportunity also exists 

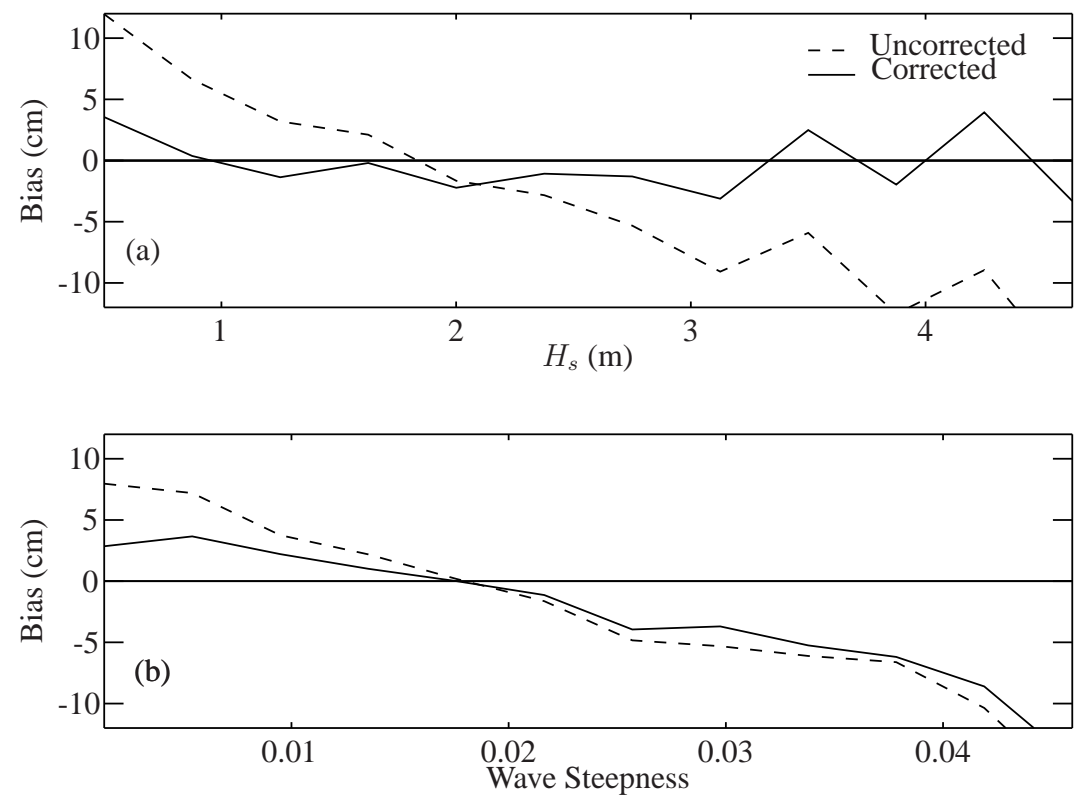

Figure 2.7: Altimeter bias as a function of (a) $H_{s}$ and (b) wave steepness for both corrected and uncorrected altimeter data (note that the uncorrected data in (b) is the same as that of Figure 2.5).

Table 2.2: Correlations between altimeter $H_{s}$ bias (altimeter minus buoy) and both $H_{s}$ and wave steepness as well as partial correlations for each variable with the effects of the other removed.

\begin{tabular}{lc}
\hline Variable & $\mathrm{R}$ \\
\hline$H_{s}$ & -0.36 \\
Steepness & -0.21 \\
$H_{s}$ with the effects of steepness removed & -0.32 \\
Steepness with the effects of $H_{s}$ removed & -0.13 \\
\hline
\end{tabular}


to extend this work to develop a correction scheme based on both wave height and wave steepness. While peak period information would be required, which is not directly reported by altimeters, there is some work investigating the use of semiempirical functions to determine period information from altimeter data (e.g. Hwang et al., 1998; Gommenginger et al., 2003; Quilfen et al., 2004; Mackay et al., 2008) which shows good agreement with buoys. These could also be obtained from first guess model estimates prior to assimilation or simply by using climatological values.

This work suggests that wave state has an influence on altimeter estimates of $H_{s}$. Though no explanation is put forward here, the hypothesis of Janssen (2000) regarding the breakdown of the assumption of a Gaussian distribution in steep wave conditions seems reasonable. The potential value of a better understanding of wave steepness effects for both validation studies, and wave model assimilation warrants further work.

\subsection{Summary}

Fast delivery $H_{s}$ data from both the Jason-1 and Envisat altimeters have been validated against in situ buoy data. Jason-1 is found to be performing consistently throughout the range of wave heights and requires no correction. Consistent with previous work, it is found to be rather noisy, certainly more so than Envisat. The RMSD between Jason-1 and buoy data is $0.227 \mathrm{~m}$. Envisat is overestimating low $H_{s}$ and underestimates high. A linear correction reduces the RMSD from 0.219 $\mathrm{m}$ to $0.203 \mathrm{~m}$, a $7 \%$ reduction. This lower corrected RMSD relative to Jason-1 is a reflection of the noise in the Jason-1 data.

A systematic difference in the $H_{s}$ being reported by MEDS and NDBC buoy networks is noted. Using the altimeter data as a common reference, it is estimated that MEDS buoys are underestimating $H_{s}$ relative to NDBC buoys by about $10 \%$.

The influence of sea-state on the accuracy of altimeter $H_{s}$ retrievals has been examined. Specifically, the relationship between altimeter $H_{s}$ bias, and wave steepness is explored. This work suggests a positive bias is present in altimeter reported $H_{s}$ for smooth conditions and a negative bias in the case of steep waves. This characteristic bias is found to be in addition to, rather than because of, $H_{s}$ dependent biases. 


\title{
Chapter 3
}

\section{An Assessment of the ACCESS Forcing Winds}

\author{
Contents

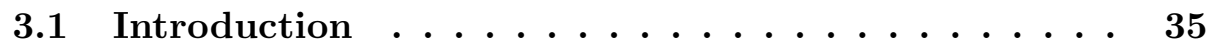 \\ 3.2 Previous Work ................ 36 \\ 3.3 Data ........................... 37 \\ 3.4 Method .................. 40 \\ 3.5 Results . . . . . . . . . . . . . . 44

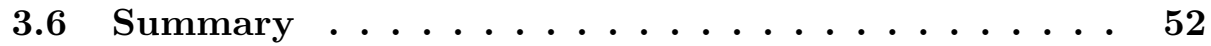

\subsection{Introduction}

In the previous chapter, Jason- 1 and Envisat altimeter $H_{s}$ observations were verified against in-situ buoys, and appropriate corrections for these data determined. These data will be used in the wave model assessments carried out in Chapter 4. Prior to any wave model verifications, an examination of the forcing winds is performed. This is the subject of this chapter.

The winds used as forcing for the wave hindcasts performed in this thesis are those from the ACCESS model. This model, based on the UK Met Office Unified Model/Variational Assimilation (UM/VAR) system (Rawlins et al., 2007), recently replaced all the existing operational NWP systems at the Bureau (i.e. 
GASP (Seaman et al., 1995) and LAPS (Puri et al., 1998)). This has led to better skill in standard NWP skill scores $(N M O C, 2010 \mathrm{a})$, however, no direct analysis of marine surface winds has been carried out.

In this chapter, the error characteristics of the $U_{10}$ from the ACCESS model will be verified against scatterometer data. This work has been carried out in the context of the overall aims of this thesis, to assess systematic error sources in a global wave model. The purpose then is simply to characterise these errors. No attempt is made to diagnose the source of identified error in the winds.

Some background is presented in Section 3.2, the data used and methods applied are discussed in Sections 3.3 and 3.4, results are presented and discussed in Section 3.5 and a summary and conclusions are given in Section 3.6.

\subsection{Previous Work}

The high quality and spatial coverage of scatterometer data makes it a valuable source of data for the study of marine surface winds (e.g. Kelly, 2004). These data have been extensively used for verification of NWP winds (e.g. Yuan, 2004; Rogers and Wittmann, 2002; Isaksen and Janssen, 2004; Chelton and Freilich, 2005) as well as in data assimilation schemes, which have been shown by many authors to have a significant positive impact on NWP accuracy (e.g. Atlas et al., 2001; Chelton et al., 2006; Perrie et al., 2008).

There have been a number of evaluations of the Bureau's previous operational marine winds using scatterometer data. Kepert et al. (2005) performed a verification of the Bureau's NWP systems that mostly focused on September 2001 and January 2002. They found that the regional model LAPS underestimated the surface winds compared with QuikSCAT by around $10 \%$. The global model, GASP was found to have a smaller bias. This work was extended by Schulz et al. (2007) who examined operational surface wind products during 2003 and 2004. They demonstrated that wind speeds from the regional model LAPS were typically underestimated by approximately $5 \%$. This study found that this bias is greater still in the global model with underestimation being approximately $5-10 \%$ in comparison to both in situ (buoy) and remote (scatterometer) data. Both studies identified a greater bias in the meridional direction than in the zonal direction. In addition, both models showed considerable seasonal variability in skill. 
The current work represents the first direct verification of the ACCESS marine surface winds, and as such provides a much needed quantification of their expected accuracy. While the previous studies of GASP discussed above have focused on overall statistics, error characteristics from NWP products are known to contain strong regional variation (e.g. Chelton and Freilich, 2005; Monahan, 2006). Hence, emphasis is placed here on examining the spatial structure of that error.

\subsection{Data}

Scatterometers are spaceborne radars that infer surface winds from the roughness of the sea surface based on measurements of ocean radar backscatter cross section, denoted as $\sigma_{0}$. This varies with wind speed, wind direction relative to the antenna azimuth, incidence angle, polarization, and radar frequency. Near-simultaneous co-located $\sigma_{0}$ measurements are acquired from different measurement geometries and polarizations, allowing wind speed and direction to be retrieved in ground processing (e.g. Naderi et al., 1991).

A general discussion of scatterometers and the interpretation of their data can be found in Stoffelen and Anderson (1997), while Stoffelen (1998) discusses the relative errors of ship, buoy, and scatterometer winds in relation to NWP verification. Scatterometer data are favoured for surface wind evaluations due to their high spatial and temporal density, as well as the fact that individual observations represent a spatial average over a horizontal scale of about $25 \mathrm{~km}$, making them less subject to errors of representativeness than point measurements (e.g. Lorenc, 1986).

This method of wind estimation is measuring the wind stress, rather than the actual $10 \mathrm{~m}$ wind speed. This distinction has important implications for interpretation of comparisons between scatterometer and NWP model winds. Firstly, scatterometer estimates of $U_{10}$ rely on an assumption of a neutrally stable atmosphere. The wind retrievals are calibrated to the equivalent neutral-stability wind at a reference height of $10 \mathrm{~m}$ above the sea surface, while NWP products are estimates of the actual $10 \mathrm{~m}$ wind. Hence, NWP winds should, strictly speaking, be adjusted for the effects of atmospheric stability for the purposes of comparison. In practise, this is rarely done. The boundary layer can be considered near neutrally stable over much of the global ocean (Chelton and Freilich, 2005), and 
so the effect of this assumption on the analysis is expected to be small. Based on stability corrections calculated from global ocean buoy measurements, Mears et al. (2000) concluded that anemometer measurements of $10 \mathrm{~m}$ winds are typically about $0.2 \mathrm{~ms}^{-1}$ lower than the equivalent neutral-stability winds at $10 \mathrm{~m}$. A similar magnitude is reported by Chelton and Freilich (2005). Overall, these effects are deemed small enough to be ignored here.

The second consideration is the effect of ocean surface currents on wind retrievals. The wind stress is determined by the vector difference between the surface wind speed and the moving ocean surface, while the model estimates wind speed relative to fixed grid points. In regions of strong mean flow, surface currents can generate differences of order $1 \mathrm{~ms}^{-1}$ between scatterometer and NWP or buoy winds (Chelton and Freilich, 2005). In the vicinity of the Pacific equatorial cold tongue, for example, Kelly et al. (2001) showed that the equatorial current system results in biases of more than $0.5 \mathrm{~ms}^{-1}$ between QuikSCAT winds and the winds measured by the Tropical Atmosphere-Ocean (TAO) moorings. Similarly, Chelton et al. (2004) showed that the surface current can modify the mean QuikSCAT winds by nearly $1 \mathrm{~ms}^{-1}$ over the core of the Gulf Stream. Again, accurate estimates of surface currents are not available in order to make adjustments to the NWP surface winds. In the absence of such adjustments, these effects must simply be accounted for in the interpretation of the results.

Wind velocity observations used in this work come from the SeaWinds scatterometer on board QuikSCAT (Lungu and Callahan, 2006). This instrument produces winds on an 1800-km-wide swath at a horizontal spacing of $25 \mathrm{~km}$, with an orbital period of 101 min, covering about $90 \%$ of the ice-free oceans per day. A single day of data is shown in Figure 3.1. QuikSCAT employs a conically scanning pencil-beam antenna, in contrast to the fan-beam antenna of the previous NSCAT (Naderi et al., 1991) and subsequent ASCAT (EUMETSAT, 2010) scatterometers. This has the advantage of a wide swath, but the disadvantage that the edges and nadir of the swath are observed from azimuths that differ by close to $0^{\circ}$ or $180^{\circ}$, rather than the optimal $90^{\circ}$, reducing accuracy in these parts of the swath (demonstrated in Chelton and Freilich, 2005).

QuikSCAT operates in the $\mathrm{Ku}$ band, and is thus more sensitive to the rain attenuation of the radar signal than the $\mathrm{C}$ band operating instruments such as ASCAT. This corruption includes both backscatter and attenuation by raindrops in the atmosphere, as well as an increase in backscatter from centimeter scale 


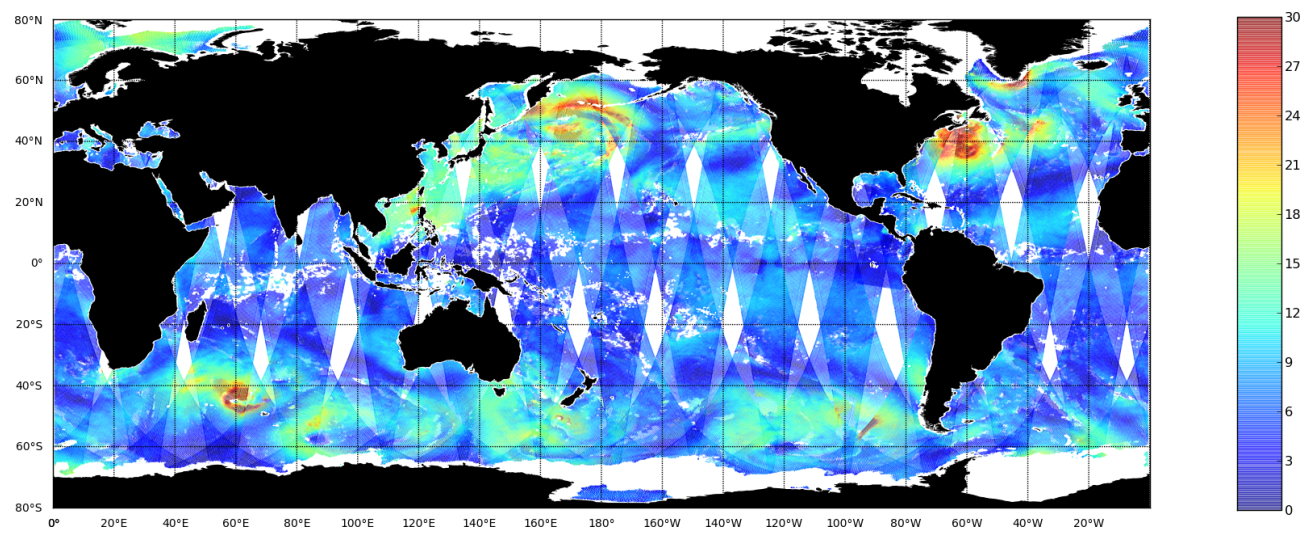

Figure 3.1: One day of QuikSCAT data showing observed $U_{10}$ in $\mathrm{ms}^{-1}$.

roughness on the sea surface caused by the impact of raindrops. Performing comparisons with buoy data, Chelton and Freilich (2005) show that directional estimations are also strongly influenced by the presence of rain at the surface, with such retrievals favouring directions perpendicular to the satellite track. A detailed discussion of rain effects can be found in this paper and references therein.

The process of determining the wind that best fits the observed radar backscatter generally produces from two to six solutions in the minimisation of a statistical cost function. These solutions are known as ambiguities, and an important part of the retrieval is to select the correct one. This process, which includes tests of the goodness of fit, neighbour checks, and comparison with NWP data, does nevertheless occasionally select an incorrect vector. Earlier scatterometers had relatively simple antenna geometries and the ambiguities were often close to $180^{\circ}$ in direction apart. However, the more complex geometry of the QuikSCAT antenna system means it is difficult to make generalisations about the nature of the ambiguities.

In the previous chapter (Chapter 2), the altimeter $H_{s}$ data used in this study were validated and (in the case of Envisat) corrected. No such validation is carried out here for the QuikSCAT observations, with validations already available in the literature deemed sufficient. The mission requirements of QuikSCAT indicate a wind speed accuracy of $2 \mathrm{~ms}^{-1}$ in wind speed for the range 3-20 $\mathrm{ms}^{-1}$ and $10 \%$ for the range $20-30 \mathrm{~ms}^{-1}$ and a directional accuracy of $20^{\circ}$ root-mean-squareerror (RMSE) for wind speeds ranging from $3 \mathrm{~ms}^{-1}$ to $30 \mathrm{~ms}^{-1}$ (Lungu and Callahan, 2006). Several studies have shown accuracies exceeding these specifications (Stoffelen, 1998; Freilich and Vanhoff, 2006, amongst others). In comparisons 
between the U.S. NDBC open-ocean in situ observations and QuikSCAT over a 2 year period, Chelton and Freilich (2005), for example, demonstrated RMSE values of around $1.7 \mathrm{~ms}^{-1}$ over all wind speeds (up to the maximum measured of $25 \mathrm{~ms}^{-1}$ ). Even at hurricane strength winds, Sharma and D'Sa (2008) suggest that QuikSCAT winds maintain accuracy comparable to that of buoys. The wind direction accuracy is a sensitive function of wind speed at low wind speeds but improves rapidly with increasing wind speed. Chelton and Freilich (2005) report a directional accuracy of about $14^{\circ}$ at $6 \mathrm{~ms}^{-1}$.

QuikSCAT data was obtained from the Center for Ocean-Atmospheric Prediction Studies $(\mathrm{COAPS})^{1}$. The product used here is the so called compact Level 2B swath product utilizing the ku-2001 geophysical model function. This product employs contemporaneous microwave radiometer measurements for additional rain flagging and sea ice detection $(R S S, 2010)$. These data are treated here as "ground truth".

The NWP surface winds examined here are from a test configuration of the global ACCESS model (identified as experiment 00ho). Grid resolution is $\sim 80$ $\mathrm{km}$ (N144). During the period examined, QuikSCAT data was assimilated in the ACCESS model, and for verification purposes, these data cannot be considered independent at the analysis time. Hence, short term forecast winds from 0-12 hour lead time are analysed. This ensures high quality winds are considered with minimal phase error, while maintaining the independence of the verification data. Model output was extracted from the Bureau's MARS archive on a regular grid at resolutions of $1^{\circ}$ at 3 hourly intervals.

\subsection{Method}

Prior to use, all rain flagged data was removed from the observational data set. Lower quality data at the edges and in the middle of the swath were not used. The swath contains 72 cells, three from each side, and six from the middle were removed. Model data is bi-linearly interpolated in space to the remaining scatterometer observation locations, and linearly interpolated in time to make up a set of co-locations. Figure 3.2 shows an example QuikSCAT pass over the East Coast of Australia before (a) and after (b) the removal of poor quality data. Figure 3.2 (c) shows a corresponding modelled $U_{10}$ field, and (d) the difference

\footnotetext{
${ }^{1}$ http://coaps.fsu.edu/
} 
between the observations and the interpolated model.

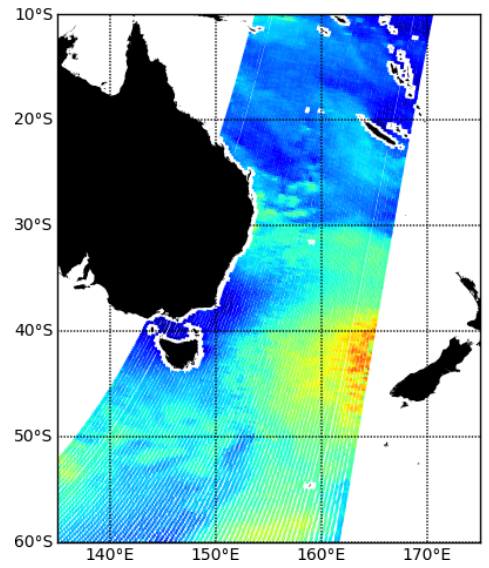

(a) Raw $U_{10}$ from QuikSCAT pass

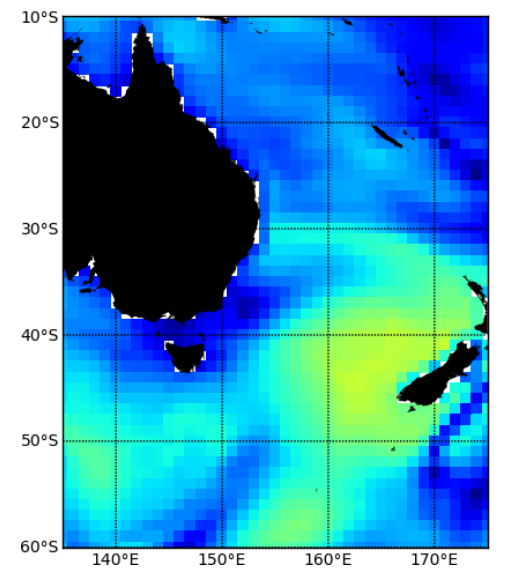

(c) Model $U_{10}$

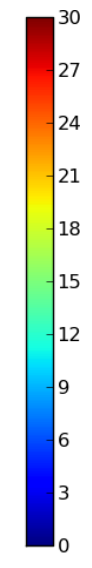

(b) Valid data used from QuikSCAT pass

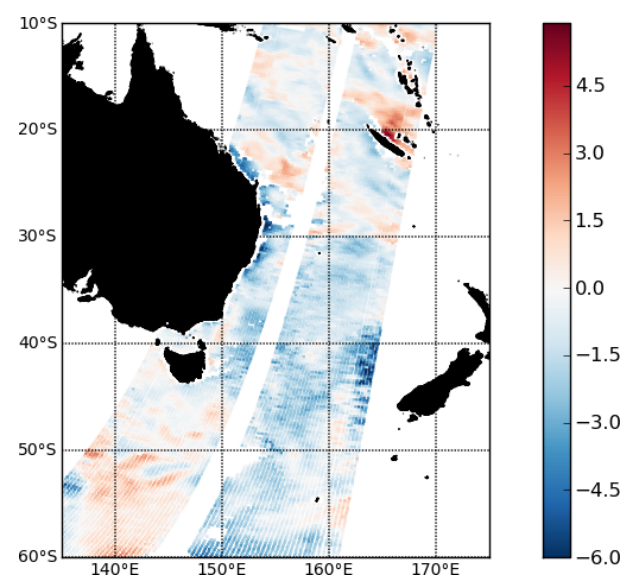

(d) Difference

Figure 3.2: Example of $U_{10}$ co-locations. QuikSCAT pass before (a) and after (b) removal of poor quality data, model data for 4 July 2008 (a) and the magnitude of the difference between co-locations calculated for this pass (d). All plots in units of $m s^{-1}$.

A four month period (July - October 2008) is examined here. This analysis resulted in more than 86 million collocations. From these collocations, various statistics can be calculated to obtain the overall error characteristics. To determine the spatial variation in error, the data are additionally binned into $1 \times 1^{\circ}$ latitude-longitude bins, and statistics are calculated for each bin separately. The choice of spatial bin size presents an inherent compromise between the desire to explore the error structure at small spatial scales, and the need to maintain sufficient data in each bin to ensure the representativeness of the calculated statistics. 
The high spatial and temporal density of the QuickSCAT swath data allows this analysis to be carried out at the relatively fine scale of $1^{\circ}$.

Figure 3.3 shows the total number of co-locations per bin for the four month period. In the major ocean basins, the distribution is primarily a reflection of the rain climatology, with minima appearing just north of the equator coinciding with the position of the Intertropical Convergence Zone (ITCZ), and in the South Pacific Convergence Zone (SPCZ) region, extending from the west Pacific warm pool south-eastwards towards French Polynesia. There are reduced numbers of colocations along coastlines and around islands. Sea ice around Antarctica results in no co-locations around this continent, with the shifting ice edge over this period resulting in a gradual reduction in the number of co-locations around the edge. It is also worth noting that the physical size of a $1^{\circ}$ box reduces at higher latitudes. However, due to the orbital characteristics of the satellite, observations density also increases at higher latitudes, maintaining sufficient observations in these smaller boxes, and in fact resulting in the maximum being located between 65 and $75^{\circ} \mathrm{N}$. Over most of the globe, there are around 3000 co-locations for each $1 \times 1^{\circ}$ bin over the four month period.

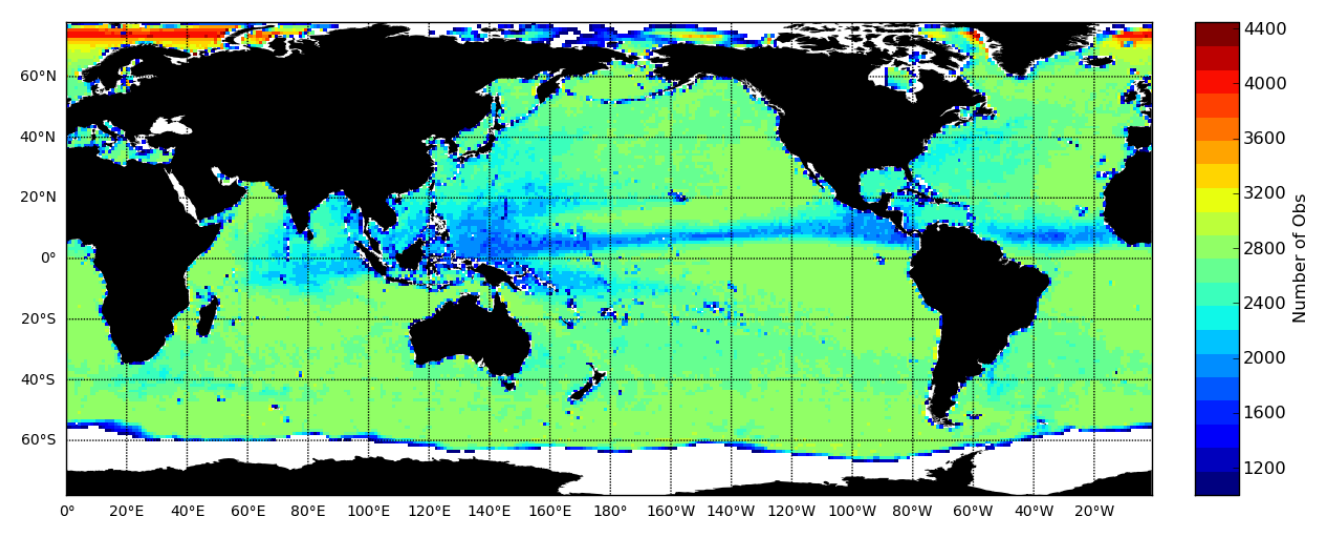

Figure 3.3: Number of QuikSCAT-model co-locations in each $1 \times 1^{\circ}$ bin.

Statistics referred to throughout this chapter are the bias, the slope of the regression line through the origin, the RMSE, the SI (standard deviation, divided by the mean observed value) and the Correlation Coefficient (R), as previously defined. Each of these measures has strengths and weaknesses. The focus here is on the bias and the SI, which gives an indication of typical scatter around this bias. It is worth noting, that in areas of persistently low wind speeds, the SI can give high values, even though the RMSE associated with these errors is small. Similarly, in such areas, small absolute biases can be associated with large 
relative biases (as indicated by the slope). In instances where presenting all these statistics is impractical, relevant commentary is made in the text.

For zonal and meridional wind components, RMSE is used instead of the SI. Though the latter has the advantage of separating error associated with bias and scatter, it becomes meaningless when considering quantities that include both positive and negative values. Slope and SI also do not make sense in the context of wind direction.

When calculating statistics from angular data such as wind direction, the 0/360 (or +180/-180) discontinuity requires special consideration. The commonly used method of Yamartino (1984) provides a means of calculating both the mean and the standard deviation from a single pass over the data. Considering $n$ measurements of wind direction $(\theta)$ two totals are accumulated without storage of the $n$ individual values:

$$
s=\frac{1}{n} \sum_{i=1}^{n} \sin \theta_{i}, c=\frac{1}{n} \sum_{i=1}^{n} \cos \theta_{i}
$$

The average wind direction is then given via the four-quadrant $\arctan (\mathrm{x}, \mathrm{y})$ function as:

$$
\theta_{a}=\arctan (c, s)
$$

From twenty different functions for using variables obtained in a single-pass of the wind direction data Yamartino (1984) found the best function to be:

$$
\sigma_{\theta}=\arcsin (\varepsilon)\left[1+\left(\frac{2}{\sqrt{3}}-1\right) \varepsilon^{3}\right]
$$

where

$$
\varepsilon=\sqrt{1-\left(s^{2}+c^{2}\right)}
$$

This method gives an estimate of the scalar average wind speed. Alternatively, vector averaging can be used. For the same sequence of wind direction observations, if the wind speed $(U)$ is also known, then the mean zonal and meridional components can be calculated as: 


$$
u_{\text {mean }}=\frac{1}{n} \sum_{i=1}^{n} U_{i} \sin \theta_{i}, v_{\text {mean }}=\frac{1}{n} \sum_{i=1}^{n} U_{i} \cos \theta_{i}
$$

From which, the mean wind speed and direction can be calculated as:

$$
\begin{gathered}
U_{\text {mean }}=\left(u_{\text {mean }}^{2}+v_{\text {mean }}^{2}\right)^{\frac{1}{2}} \\
\theta_{\text {mean }}=\arctan \left(u_{\text {mean }}^{2}, v_{\text {mean }}^{2}\right)
\end{gathered}
$$

The unit vector approach of computing mean wind direction is similar to the vector mean described above except that the east-west and north-south components are not weighted by the wind speed. In general, the direction calculated using a unit vector approach will be comparable to the scalar average wind direction. Unless otherwise stated, vector averaging is used here.

\subsection{Results}

Global statistics for the four months for wind speed, wind direction, $u$ and $v$ are given in Table 3.1. A large negative bias is apparent, reflected in both the bias and the slope. For the components, bias is harder to interpret due to the fact that these variables include both positive and negative values. From the slope, as with previous studies of GASP winds, the underestimation is more prevalent in the meridional component than the zonal component.

Table 3.1: ACCESS $U_{10}$ verification statistics against QuikSCAT data for the period July to October 2008.

\begin{tabular}{lrrrr}
\hline & Bias & RMSE & SI & slope \\
Speed $\left(m s^{-1}\right)$ & -0.53 & 1.57 & 0.18 & 0.92 \\
Direction $\left(^{\circ}\right)$ & -0.36 & 24.8 & & \\
$u\left(m s^{-1}\right)$ & 0.05 & 1.78 & & 0.91 \\
$v\left(m s^{-1}\right)$ & -0.05 & 1.83 & & 0.85 \\
\hline
\end{tabular}

A scatter plot of observed $U_{10}$ against modelled is presented in Figure 3.4, with counts per bin for $0.5 \times 0.5 \mathrm{~ms}^{-1}$ bins indicated. Some care must be applied when interpreting scatter plots using this amount of data, as outliers can create the impression of a large spread. Data densities are plotted here on a logarithmic 
scale, and only bins containing more than 100 observations shown. In general, the model does a reasonable job in the $0-20 \mathrm{~ms}^{-1}$ range, while a large departure from the observations can be seen above $25 \mathrm{~ms}^{-1}$. The lack of ability to capture these extreme values is likely due in a large part to model resolution (e.g. Cavaleri, 2009). Less than $0.3 \%$ of the QuikSCAT observations are above $25 \mathrm{~ms}^{-1}$, and these don't have a large impact on the verification statistics presented. This deficiency is, however, of note due to relevance for severe weather forecasts in general, and the increased relative impact of these high winds on wave growth in particular here.

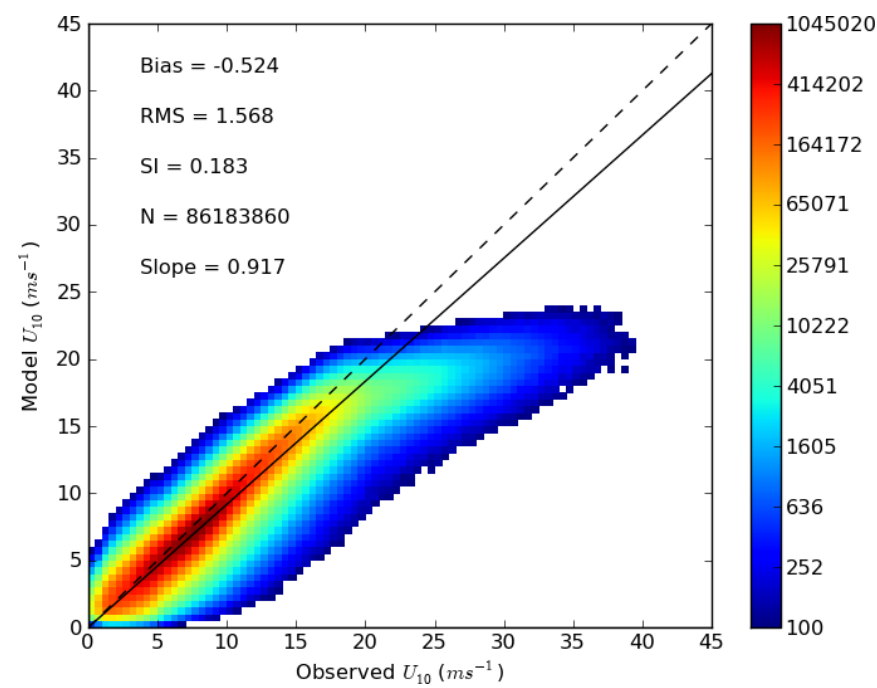

Figure 3.4: Scatter plot of $U_{10}$ based on comparisons with QuikSCAT data for the period July to October 2008. Data are density in $0.5 \times 0.5 \mathrm{~m}$ bins are shown on a logarithmic scale.

Figure 3.5 shows wind speed probability density functions for modelled and observed wind speeds, constructed from this same set of co-locations. Overall, it is clear that the model distribution is shifted down the wind range relative to the observations. Model and observations densities appear similar around 15-16 $\mathrm{ms}^{-1}$. Rather than an indication that the model is performing notably well in this range, this is likely a result of the increasingly negative bias above it. Under-predicted $U_{10}$ at extreme values increase densities at these moderately high wind speeds, locally offsetting decreases due to the general down shift across the full range.

While these statistics over the full domain provide information about the overall quality of the winds, greater insight can be gained from a knowledge of the regional variation of these error characteristics. To provide some context 


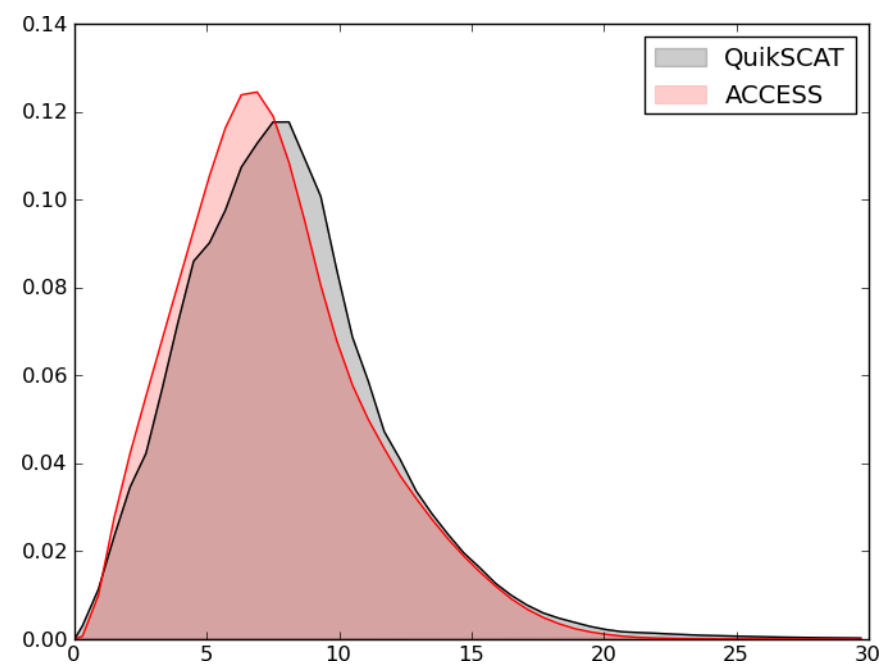

Figure 3.5: Probability density function for modelled and observed wind speeds.

for spatial error plots, Figure 3.6 (a) shows the mean wind speed $\left(\bar{U}_{10}\right)$ and direction over the globe calculated from all QuickSCAT observations over this period. Large values of westerly $\overline{U_{10}}$ values occur in the Southern Hemisphere mid-latitudes, coincident with the winter storm tracks. Though a westerly belt is apparent in the Northern Hemisphere, it is modest. Secondary maxima in the easterly belts occur in both hemispheres on the equatorward flanks of the subtropical highs. Minima occur in the equatorial convergence zones and the subtropical highs.

Standard deviations of the observed wind speed $\left(\sigma_{U_{10}}\right)$ and direction $\left(\sigma_{U_{\theta}}\right)$ are shown in Figures 3.6 (b) and (c) respectively, with the latter being calculated using the method of Yamartino (1984) discussed above. Maxima in wind speed variability occur in the mid-latitude storm tracks, with minima occurring in the eastern Equatorial Pacific and Equatorial Atlantic. Maxima in the directional variation can be seen in the tropical convergence zones as well as in the tracks of the subtropical highs, coincident with the transition from westerly mid-latitude to easterly extra-tropical mean flow. This is likely enhanced by the expected increase in directional error and increased likelihood of incorrectly chosen ambiguities in the scatterometer data in these low wind speed conditions.

Figure 3.7 shows the spatial distribution of the error in the wind speed, calculated as described in section 3.4. Though the interest here is primarily on the large scales, there are many small scale features worth noting. There are clear errors associated with the transition from land to sea. Both the bias and the SI 


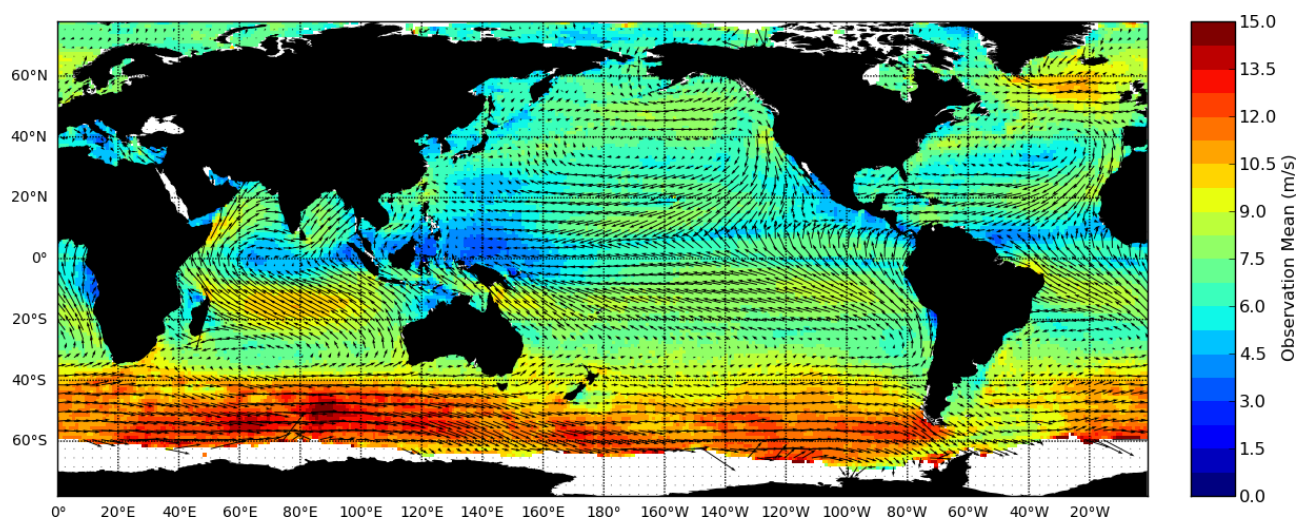

(a) Observed mean wind speed $\left(m s^{-1}\right)$ and direction

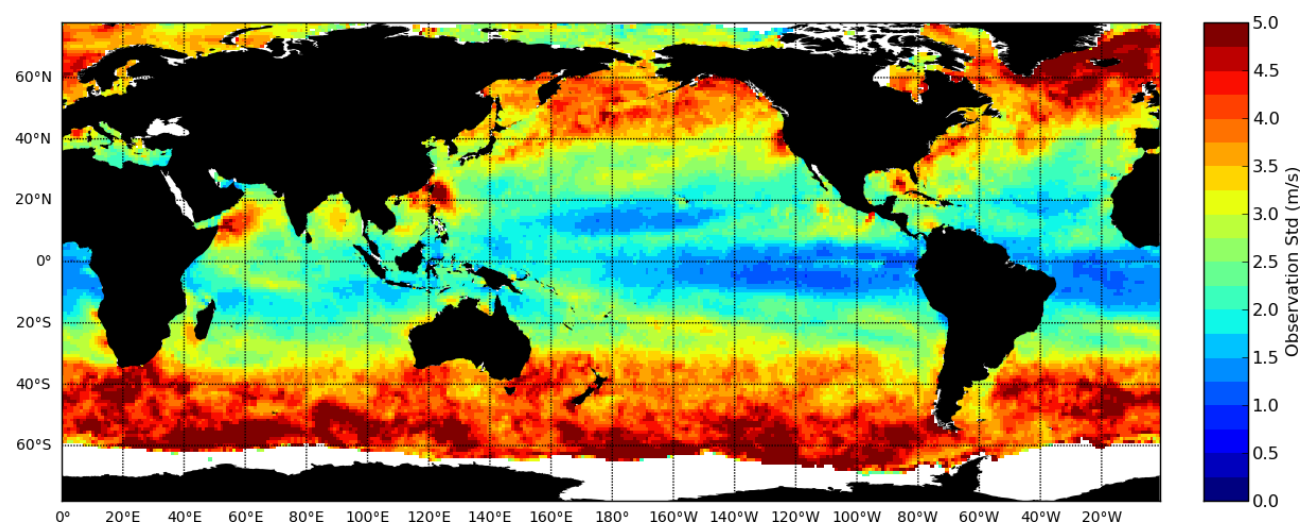

(b) Wind speed standard deviation $\left(m s^{-1}\right)$

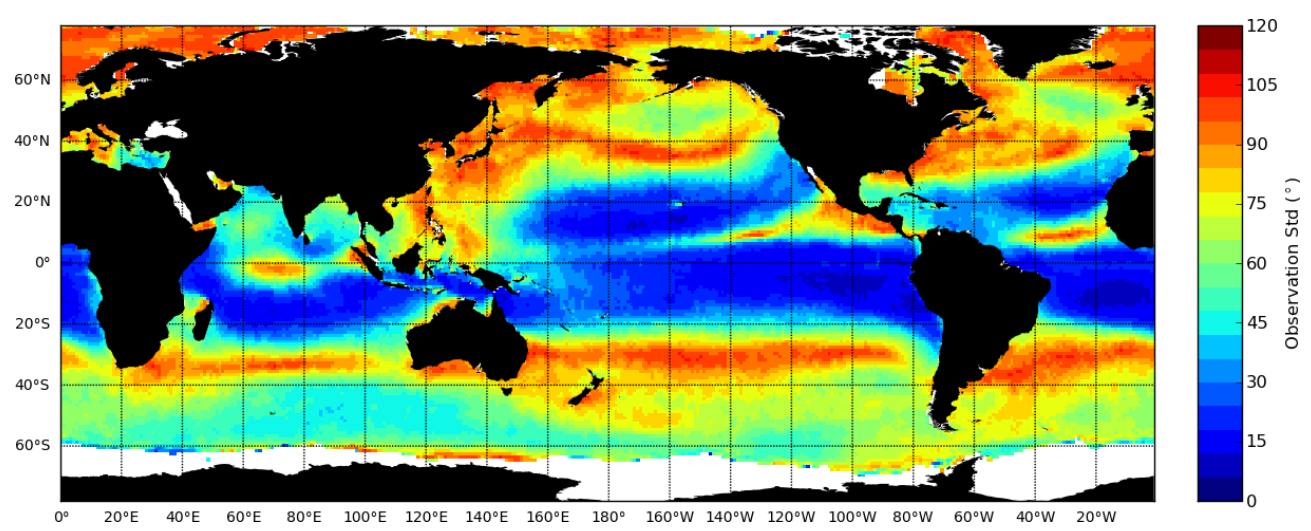

(c) Wind direction standard deviation $\left(^{\circ}\right)$

Figure 3.6: $U_{10}$ statistics based on QuikSCAT data for the period July to October 2008. 
exhibit large values near islands, especially in the Tropics. These differences are mostly attributable to wind shadows that are resolved by the QuikSCAT observations (e.g. Xie et al., 2001; Chelton et al., 2004), but are poorly represented by NWP models (e.g. Chelton and Freilich, 2005; Kara et al., 2008).

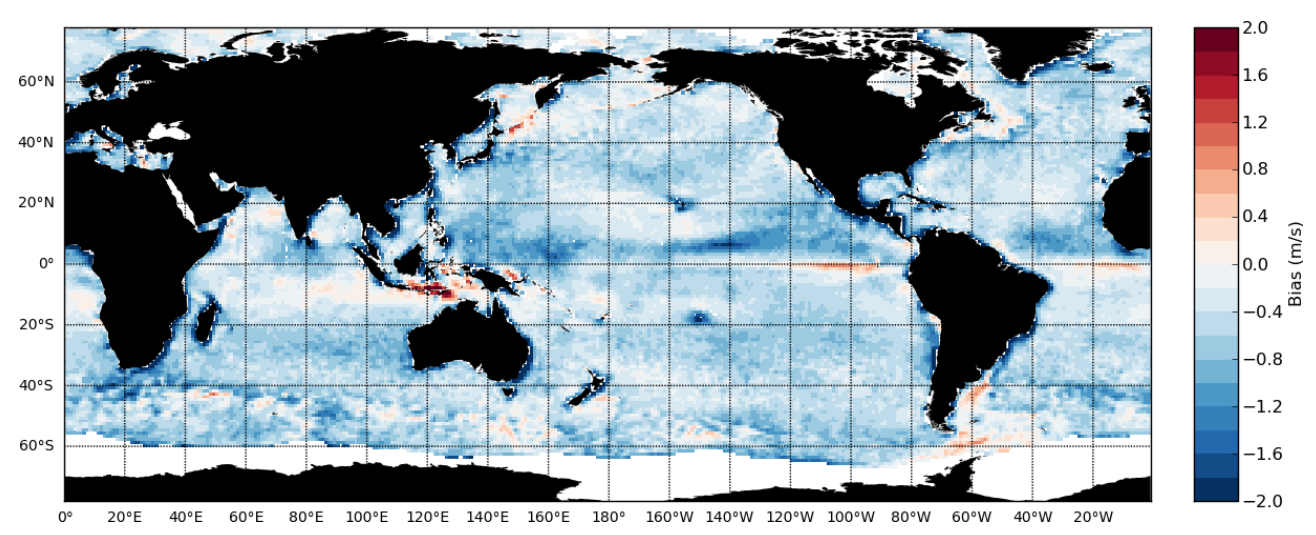

(a) Bias

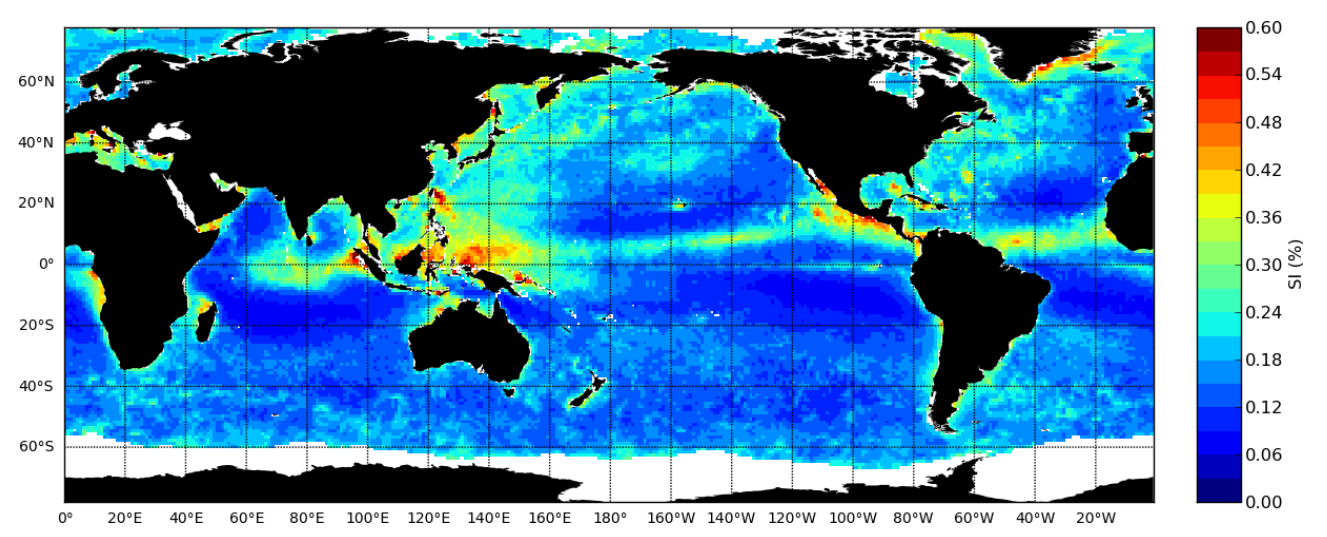

(b) SI

Figure 3.7: ACCESS $U_{10}$ bias and SI based on comparisons with QuikSCAT data.

Similar features can be seen along the edge of continents and at the Antarctic ice edge. These local biases are particularly significant on the east coasts of the major Southern Hemisphere landmasses. Figure 3.8 for example, shows the severity of this bias in more detail on the Australian east coast. While the poor ability of the model to capture the complex transition from land to sea is also likely playing a role here, the existence of strong boundary currents in these regions could also be influential. This is discussed further in Chapter 6 .

Large values of the SI can be seen to the west of the mountain gap of Tehuantepec in Mexico on the Isthmus of Tehuantepec in Figure 3.7, representing the 


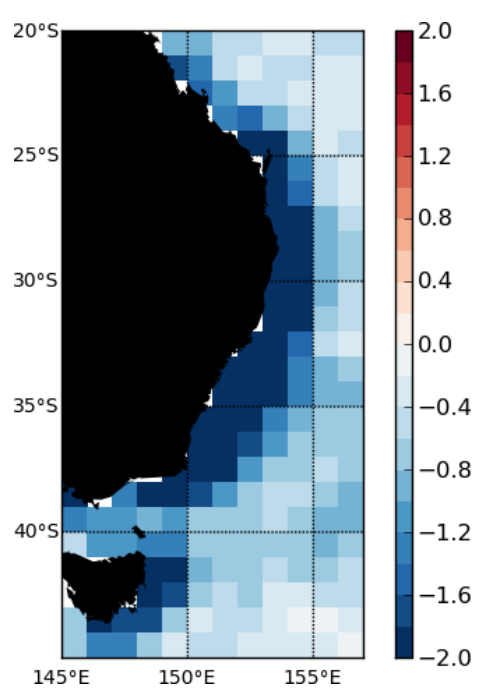

(a) Bias

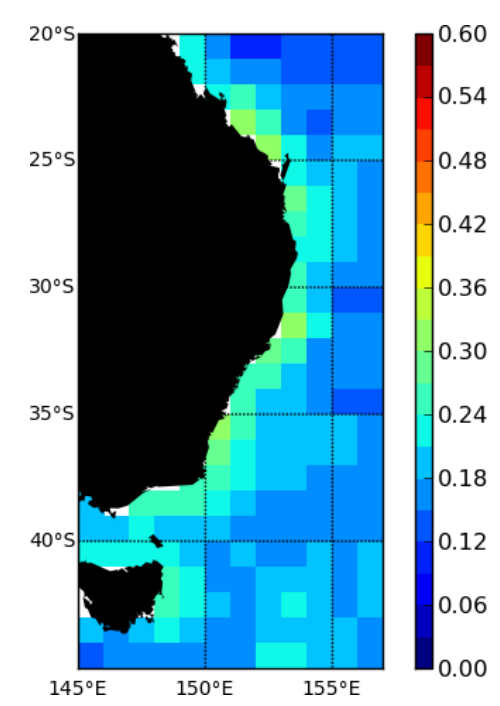

(b) SI

Figure 3.8: Same as Figure 3.7 for the Australian east coast.

shortest distance between the Gulf of Mexico and the Pacific Ocean. This region is famous for producing strong easterly winds that are channelled by topography (e.g. Xie et al., 2005; Sun and $Y u, 2006$ ). Such features are poorly resolved at this model resolution. Small scale features can also be seen in the Southern Ocean, most notably south of the Indian Ocean. These are consistent with the findings of Chelton et al. (2004) who identified similar patterns in the wind stress and divergence fields recontructed from 4 years of QuikSCAT data. They postulated that these persistent features are the result of Sea Surface Temperature (SST) fronts. The fact that they are present in these model/observations difference plots is likely due both to the stability effects that the SST has on the scatterometer data, as well as the fact that these features are inadequately captured by the model.

On the larger scale, high scatter is apparent just north of the equator in the region of the ITCZ, and over large regions to the east (Pacific Ocean) and to the west (Indian Ocean) of Indonesia. This is due primarily to low wind speeds in these areas (refer to Figure 3.6 (a)). Notable structure can be seen in regions of strong currents: the Agulhas Return Current in the southwest Indian Ocean, the Gulf Stream in the North Atlantic, and the Kuroshio Extension in the North Pacific. Though these currents contribute to the existence of SST fronts, this is also attributable, in part, to the fact that scatterometers measure winds relative to a moving sea surface while NWP models produce winds relative to fixed grid 
points, as discussed in Section 3.3. Overall, significant negative bias is apparent over much of the globe. Within this general negative bias, significant spatial structure is apparent.

Statistics calculated separately for each of the four months examined here are presented in Table 3.2, indicating little variation from month to month. The spatial distribution of the bias, shown in Figure 3.9, appears similarly stable on the large scale, though some variation is apparent on smaller scales. This indicates a high degree of consistency in the wind error across the four months examined here. However, the stability of the wind error characteristics over longer periods is unknown and can be expected to show variation on seasonal and longer timescales.

Table 3.2: ACCESS $U_{10}$ verification statistics against QuikSCAT data for each month of the period examined based on comparisons with QuikSCAT data.

\begin{tabular}{lrrrr}
\hline Run & Bias & RMS & SI & Slope \\
\hline July & & & & \\
Speed $\left(m s^{-1}\right)$ & -0.51 & 1.53 & 0.18 & 0.92 \\
Direction $\left(^{\circ}\right)$ & -0.22 & 24.8 & & \\
$u\left(m s^{-1}\right)$ & 0.03 & 1.76 & & 0.90 \\
$v\left(m s^{-1}\right)$ & -0.06 & 1.78 & & 0.87 \\
\hline August & & & & \\
Speed $\left(m s^{-1}\right)$ & -0.52 & 1.60 & 0.18 & 0.91 \\
Direction $\left(^{\circ}\right)$ & -0.33 & 25.0 & & \\
$u\left(m s^{-1}\right)$ & 0.03 & 1.76 & & 0.90 \\
$v\left(m s^{-1}\right)$ & -0.08 & 1.80 & & 0.86 \\
\hline September & & & & \\
Speed $\left(m s^{-1}\right)$ & -0.53 & 1.58 & 0.18 & 0.92 \\
Direction $\left(^{\circ}\right)$ & -0.42 & 25.24 & & \\
$u\left(m s^{-1}\right)$ & 0.07 & 1.79 & & 0.91 \\
$v\left(m s^{-1}\right)$ & -0.05 & 1.85 & & 0.85 \\
\hline October $_{\text {Speed }\left(m s^{-1}\right)}$ & -0.53 & 1.60 & 0.18 & 0.92 \\
Direction $\left(^{\circ}\right)$ & -0.46 & 24.30 & & \\
$u\left(m s^{-1}\right)$ & 0.09 & 1.81 & & 0.92 \\
$v\left(m s^{-1}\right)$ & -0.03 & 1.87 & & 0.84 \\
\hline
\end{tabular}

Finally, directional biases are shown in Figure 3.10. Clear banding can be seen associated with the large scale latitudinal circulations. Locally large errors are present in the mid-latitude divergence zones. These are likely associated with 


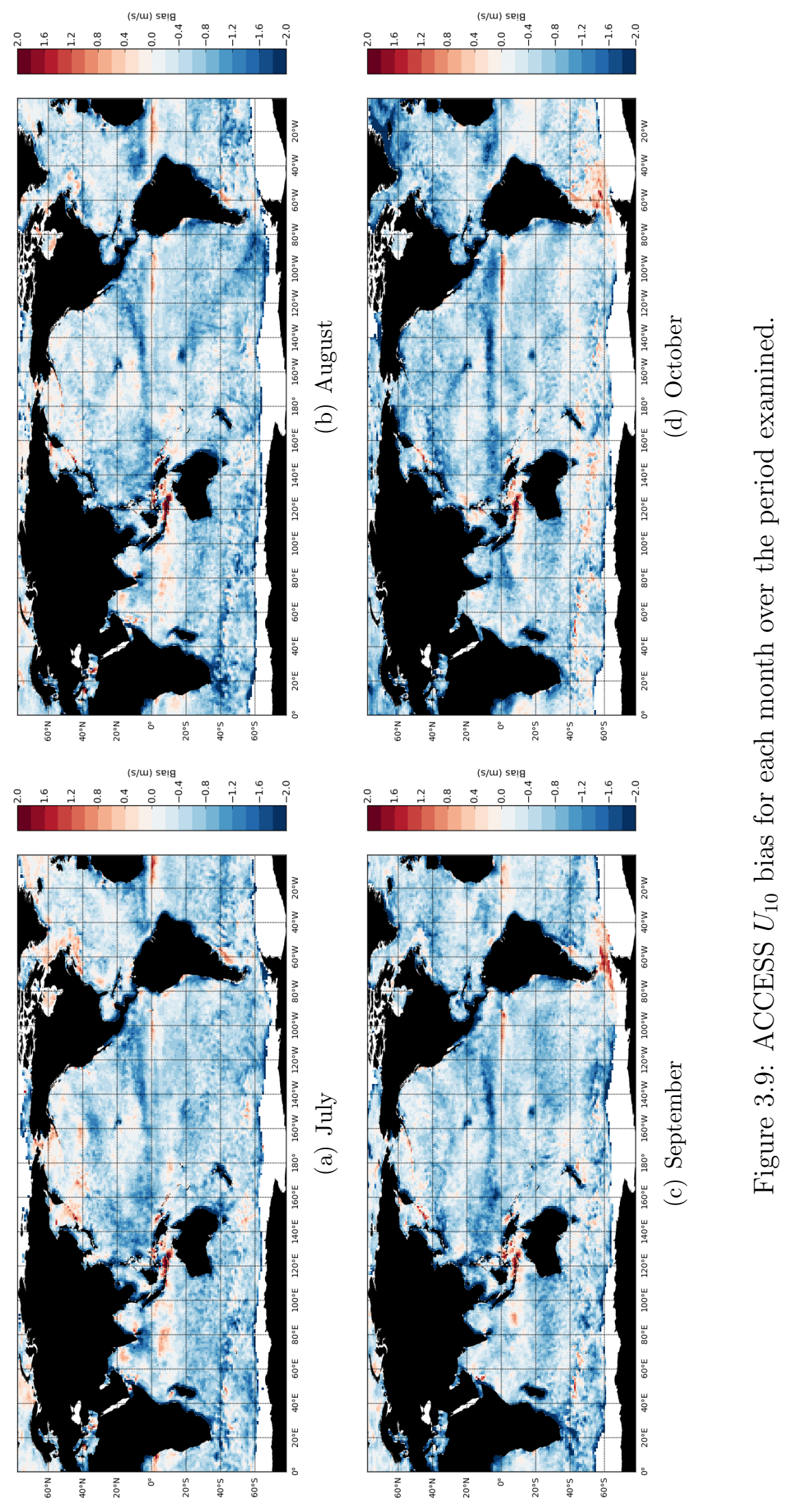


slight misplacement of these features, though large directional uncertainty in both the model and observations associated with low wind speeds in these areas is also expected.

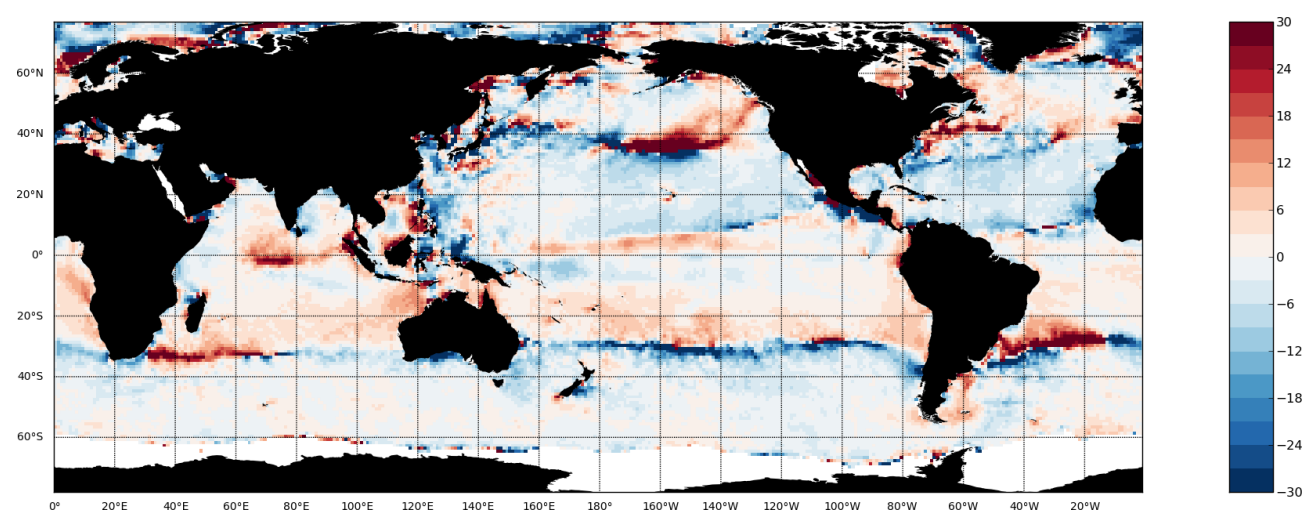

Figure 3.10: ACCESS wind direction bias $\left({ }^{\circ}\right)$ based on comparisons with QuikSCAT data for the period July to October 2008.

\subsection{Summary}

This chapter has presented the first direct assessment of the ACCESS marine surface winds against QuikSCAT scatterometer data. A low bias has been identified, with $U_{10}$ underestimated by approximately $8 \%$. Significant regional variation is also apparent. These results emphasize the importance of examining the spatial structure of model error, especially in the context of downstream systems. It is in this context that the wave model verifications are carried out in the following chapter.

Possible explanations for the differences between scatterometer observations and the NWP marine surface winds include the limited spatial resolution of the models, deficiencies in the atmospheric model physics, inadequacies in the model parameterizations of the atmospheric boundary layer response to SST, and inaccuracies in the specification of the SST boundary condition. Though out of scope of this current work, further investigation of the origin of these errors is warranted. 


\section{Chapter 4}

\section{Set Up and Verification of WAVEWATCH III}

\section{Contents}

4.1 Introduction . . . . . . . . . . . 53

4.2 The Wave Model . . . . . . . . . . . . . 54

4.3 Data and Method ............... 58

4.3 .1 Model Set Up . . . . . . . . . . . . . . . . . . 58

4.3 .2 Model Runs . . . . . . . . . . . . . . . . . . . . . . 60

4.3.3 Verification Method ............... 61

4.4 Results .................... 66

4.4 .1 Source Terms . . . . . . . . . . . . . . . 66

4.4 .2 Numerics . . . . . . . . . . . . . . . . . 72

4.4.3 Sub-Grid-Scale Blocking . . . . . . . . . . . 76

4.5 Summary .................... 79

\subsection{Introduction}

In Chapter 2, Jason-1 and Envisat altimeter $H_{s}$ observations were verified against in-situ buoys, and appropriate corrections for these data determined. The forcing winds used in this work, those of the ACCESS model, were then assessed in comparisons with QuikSCAT scatterometer observations in Chapter 3. This chapter 
discusses the set up of the numerical wave model and the verification methods used to evaluate the model runs throughout the remainder of this thesis. The verification results from a number of global runs using the ACCESS forcing are then presented. Verification data include buoy data, and altimeter data, the latter corrected according to the previously determined corrections. Validation results are discussed in the context of the now known error characteristics of the forcing winds.

The wave model used in this work is the recent release of the WAVEWATCH III $^{\circledR}$ (WW3) model, version 3.14 (Tolman, 2009). Some background on WW3 is given in Section 4.2, and the model set up, data sources and verification methods are described in Section 4.3. Results are presented in Section 4.4 and a summary and conclusion are given in Section 4.5.

\subsection{The Wave Model}

WW3 is primarily developed at NCEP, where it has been the operational system since March 2000 ( Tolman et al., 2002). The open, pluggable nature of the WW3 code has resulted in a large growth in the use of this model in the international wave model community, both amongst research groups and operational centres. The recently released version 3.14 of the model, has been made freely available by request to $\mathrm{NCEP}^{1}$. The description given below is necessarily brief, full details of the model can be found in the user manual (Tolman, 2009) and references therein.

The prognostic variable used within WW3 is the wavenumber spectrum $(F(k, \theta))$, as opposed to the frequency spectrum used by WAM $(F(f, \theta))$. The wavenumber spectrum is chosen due to its invariance characteristics with respect to the physics of wave growth and decay for variable water depths. The WW3 model solves the linear balance equation for the spectral wave action density $N$ in terms of wavenumber $k$ and wave direction $\theta$, as a slowly varying function of space $\mathbf{x}$ and time $t$ :

$$
\frac{D N(k, \theta, \mathbf{x}, t)}{D t}=\mathrm{S}(k, \theta, \mathbf{x}, t)
$$

This is closely related to the wave transport equation solved within WAM

\footnotetext{
${ }_{1}^{1}$ http://polar.ncep.noaa.gov/waves/wavewatch/wavewatch.shtml
} 
(Equation 1.1), with the action density spectrum $N$ relating to the energy density spectrum $F$ as $N=F / \sigma$, where $\sigma$ is the relative or intrinsic wave frequency (= $\left.2 \pi f_{r}\right)$. Similarly, $\mathbf{S}=S / \sigma$. The intrinsic frequency is related to the wavenumber through the dispersion relation:

$$
\sigma^{2}=g k \tanh k d
$$

where $d$ is the mean water depth. The intrinsic frequency is related to the absolute frequency $\omega\left(=2 \pi f_{a}\right)$, as observed in a fixed frame of reference, through the Doppler equation:

$$
\omega=\sigma+\mathbf{k} \cdot \mathbf{U}
$$

where $\mathbf{U}$ is the mean current velocity vector. The use of the action balance equation in place of the wave transport equation allows for the effect of large scale currents on the evolution of the wave spectrum (see Tolman, 1991, for detailed discussion). When currents are absent, or not considered, as is the case in the work presented here, equations 1.1 and 4.1 are essentially identical.

The model output consists of the more traditional frequency-direction spectrum, calculated using the Jacobian transformations:

$$
\begin{gathered}
F\left(f_{r}, \theta\right)=\frac{\partial k}{\partial f_{r}} F(k, \theta)=\frac{2 \pi}{c_{g}} F(k, \theta) \\
F\left(f_{a}, \theta\right)=\frac{\partial k}{\partial f_{a}} F(k, \theta)=\frac{2 \pi}{c_{g}}\left(1+\frac{\mathbf{k} \cdot \mathbf{U}}{k c_{g}}\right)^{-1} F(k, \theta) \\
c_{g}=\frac{\partial \sigma}{\partial k}=n \frac{\sigma}{k}, n=\frac{1}{2}+\frac{k d}{\sinh 2 k d}
\end{gathered}
$$

where $c_{g}$ is the wave group velocity. For any of these spectra, one dimensional spectra can be generated by integration over direction, with integration over the entire spectrum giving the total variance $E$, usually referred to as the total energy, i.e.

$$
E=\iint F\left(f_{r}, \theta\right) d f d \theta
$$


$H_{s}$ is then closely approximated as (e.g. Longuet-Higgins, 1952):

$$
H_{s}=\sqrt{4 E}
$$

Equation 4.1 is solved using a fractional step method, where parts of the equation are solved consecutively. Divisions include separate calculations for spatial propagation, intraspectral propagation, and source terms. The equations are solved by marching forward in time with an overall global model time step $\Delta t_{g}$, while fractional time steps are allowed to vary depending on the net source term $\mathrm{S}$, a maximum change of action density $\Delta N$ and the remaining time in the interval $\Delta t_{g}$. This technique offers improvements in both efficiency and accuracy (Tolman, 1992).

The spatial propagation equation is solved using the ULTIMATE QUICKEST (UQ) scheme and limiter of Leonard (Leonard, 1979, 1991), which is third order accurate in both time and space. The implementation of this scheme in WW3 is discussed in Tolman (1995). Importantly for the large scale error examined here, numerical diffusion is reduced relative to first order schemes (such as that used in WAM).

Reducing numerical diffusion, however, also has the effect of increasing occurrence of the so-called Garden Sprinkler Effect (GSE). This numerical feature is present when the discretization of the spectrum is too coarse for the scale of propagation, causing non-physical discontinuities in the wave field as natural dispersion occurs (e.g. WAMDIG, 1988). With higher order propagation schemes, the GSE is unfortunately more apparent, as numerical diffusion, though erroneous, has the positive quality of tending to counteract the GSE, smoothing these discrete features together. This numerical diffusion is unrelated to physical dispersion however, and should preferentially not be relied upon to mimic the natural dispersion of continuous spectra.

Thus, a controllable diffusion was introduced into WW3, in the form of a diffusion correction term to the propagation following Booij and Holthuijsen (1987). In the most recent release, a grid point averaging technique has also been implemented that produces nearly identical results at lower computational cost, particularly at high resolutions (Tolman, 2002b). This is now the default GSE alleviation method, and that used in this work.

Another important numerical feature of WW3 on the large spatial scales 
considered in this work, is the ability to account for sub-grid-scale blocking on wave propagation. At the typical resolutions of operational wave models, there are many islands that are too small to be explicitly resolved by the grid. The blocking of wave energy by these unresolved islands and reefs can be a significant source of error in wave prediction models. Accounting for these obstructions explicitly requires high grid resolutions, which are not feasible in an operational environment. Alternatively, such features can be modelled as sub-grid obstructions (e.g. Hardy, 2001). In WW3, a methodology from the SWAN model (Booij et al., 1999; Holthuijsen et al., 2002) was adopted to apply the effects of unresolved obstacles at the cell boundaries of the spatial grid within the numerical scheme. In this approach, the numerical fluxes between cells through their common boundary are suppressed according to the degree of obstruction provided by the unresolved obstacle (Tolman, 2003).

As discussed previously, the deep water source terms of relevance here consist of $S_{i n}, S_{n l}$ and $S_{d s}$, often collectively termed the model 'physics'. The current release of WW3 contains a number of different source term options. In addition to the default formulation of Tolman and Chalikov (1996), those of WAM3 (Snyder et al., 1981; Komen et al., 1984) and WAM4 (Janssen, 1991), briefly described in Chapter 1, can also be used within the WW3 model framework.

Wind and current input data are updated at the end of every time step $\Delta t_{g}$. Interpolation of speed and direction is done linearly in time by default, but wind speed can optionally be interpolated quadratically in time (energy conserving) or ingested with no interpolation and changed discontinuously. Time varying sea ice concentration can also be ingested, with two methods for ice treatment are available. In the first, where the input ice concentration for a given grid point exceeds a user defined threshold, these points are removed from the calculation, and boundary conditions identical to boundary conditions at the land boundaries are imposed. When ice concentration falls below this threshold, the grid point is reactivated, and initialised with a Pierson-Moskowitz (PM) spectrum based on the local wind. Alternatively, a continuous method is also available, as described by Tolman (2003). Utilizing the framework of the modeling of unresolved obstacles as discussed above, evolving obstruction levels are calculated based on sea ice concentration values, with threshold values for no obstruction and complete obstruction, set by the user. 


\subsection{Data and Method}

\subsubsection{Model Set Up}

The following section describes the set up of the WW3 model. The specifics outlined here are common to all hindcast performed in this thesis.

The model is run on a global grid at $1^{\circ}$ spatial resolution, matching that of the forcing winds. The wave spectrum is resolved into 24 azimuthal direction bins and 25 frequency bins logarithmically spaced from 0.04118 to $0.4461 \mathrm{~Hz}$. Directional bins are also rotated by half a bin to avoid excessive propagation of energy along the grid axes (Bidlot, 1997; Greenslade, 2000). WW3 requires four different time steps. The 'global' time step, by which the entire solution is propagated in time, the spatial propagation time step, the intra-spectral propagation and the minimum source term integration time step. The minimum source term integration time step refers to the fact that the actual time step within the model is dynamically adjusted as described previously. The time steps are determined based on efficiency and numerical stability considerations. Global, spatial propagation, the intra-spectral propagation and minimum source term integration time steps are set at 3000, 1000, 1500, and 15 seconds respectively.

A number of global bathymetry data sets were available from which to construct the depth grid:

1. ETOPO2, produced by National Geophysical Data Center $(N G D C, 2006)$.

2. DBDB2v3, produced by the National Research Labs $(N R L, 2006)$. This data set incorporates the Australian Bathymetry and Topography Grid produced by Geoscience Australia (Petkovic and Buchanan, 2002, hereafter GA2002).

3. BLUElink global bathymetry, the data set used by the operational ocean model at the Bureau. This is essentially DBDB2v2 using GA2002 in the Australian Region.

4. The Smith and Sandwell global data set, a high resolution bathymetric data set derived by combining available depth soundings with high-resolution marine gravity information from the Geosat and ERS-1 satellites (Smith and Sandwell, 1997). 
Wind waves are generally considered to by unaffected by the ocean floor where the depth is greater then half the wavelength. Taking a typical wave length to be say $100 \mathrm{~m}$ ( 8 second period), bathymetric accuracy only becomes relevant at around $\sim 50 \mathrm{~m}$. Hence, for Bureau operations, it is the accuracy of the data in Australian coastal regions that is of primary interest. To gauge this, comparisons are made with known buoy depths along the Australian coast. The complete list of buoys used is listed in Appendix C. Validations shown in Figure 4.1 are calculated from buoys in water depths less than $50 \mathrm{~m}$ only. Note that ETOPO2 was found to perform poorly in the Australian region, and is omitted here. These simple validations suggest that DBDB2v3 is the best data set in the Australian region, though it is difficult to draw definitive conclusions based on these relatively few points of comparison. The DBDB2v3 data set also has the advantage that it extends to $90^{\circ} \mathrm{N}$ and $90^{\circ} \mathrm{S}$, compared to $76^{\circ}$ for the BLUElink data, making the former the logical choice here. The global depth grid calculated from these data is shown in figure 4.2 , extending to $\pm 78^{\circ}$ latitude.

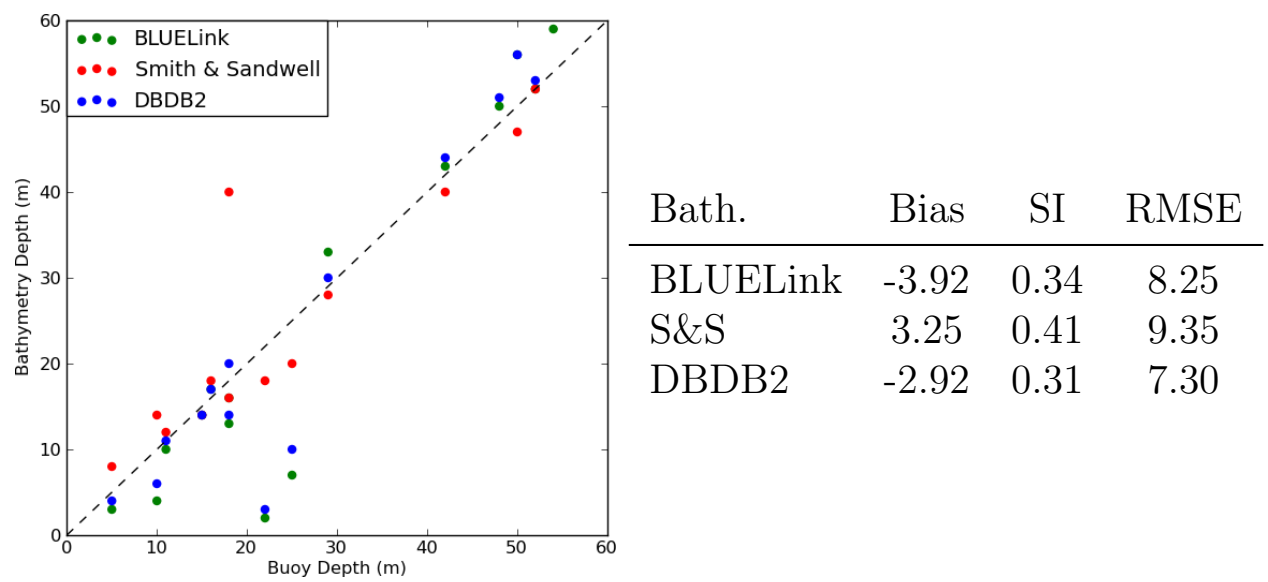

Figure 4.1: Validations of bathymetry data sets at known buoy depths around the Australian coast.

As discussed in Section 4.2, WW3 has the ability to account for blocking occurring due to islands that are too small to be resolved explicitly. This requires the input of obstruction grids, indicating the percentage of each grid cell blocked in both the $\mathrm{x}$ and $\mathrm{y}$ directions. The construction of these grids has previously been a manually intensive task. Recently, an algorithm has been developed at NCEP to construct these grids automatically (Chawla and Tolman, 2008). The algorithm accounts for islands overlapping grid cells as well as the orientation of islands in neighboring cells. In addition to a bathymetry data set, the Global Self-consistent Hierarchical High-resolution Shoreline (GSHHS) coastline dataset 


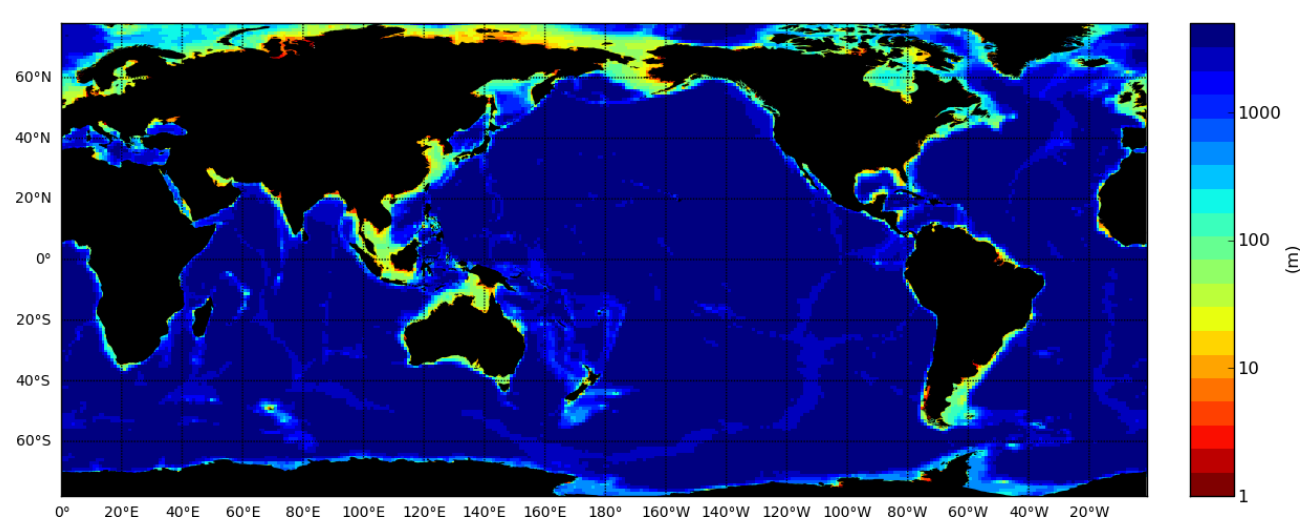

Figure 4.2: Bathymetry (m) derived from DBDB2 version 3.0 for the global grid on a logarithmic depth scale.

(Wessel and Smith, 1996) is used, which resolves small islands and structures that are beyond the capability of the bathymetry. The GSHHS data set, for example, contains 180,509 coastal boundaries, 99\% of which have areas less than $6 \mathrm{~km}^{2}$. By comparison, the 2' global bathymetry used here has a resolution of $\sim 14 \mathrm{~km}^{2}$. Further details about how the obstruction grids are constructed can be found in Chawla and Tolman (2008), while technical detail on the use of the code is given in Chawla and Tolman (2007).

Sea ice is accounted for directly using the daily SSMI high resolution sea ice concentration data produced by NCEP (Grumbine, 1996) and updated daily. The continuous ice treatment method of Tolman (2003) is used, with default threshold concentration levels $\left(\epsilon_{c, 0}=\epsilon_{c, n}=0.5\right)$.

\subsubsection{Model Runs}

The hindcasts performed and analysed in the chapter aim to assess, in a broad sense, the relative performance of various model options. The focus here is on the choice of source terms, but the impact of propagation numerics and subgrid-scale blocking are also considered. A list of runs performed is presented in Table 4.1, and described further in the following text. In each case, runs span the four month period over which the wind verifications of Chapter 3 were performed, from July 2008 to October 2008. Default parameterisations are used for all source term options. Each run was initialised using JONSWAP spectra and spun up for the full month of June. 
Table 4.1: Hindcasts performed and analysed in this chapter.

\begin{tabular}{lllc}
\hline Name & Source Terms & Blocking & Numerics \\
& & & \\
\hline TC96 & Tolman and Chalikov (1996) & Yes & UQ \\
WAM3 & Komen et al. $(1984)$ & Yes & UQ \\
BAJ & Bidlot et al. $(2007)$ & Yes & UQ \\
BAJ-1st & Bidlot et al. $(2007)$ & Yes & $1^{\text {st }}$ order \\
BAJ-NoSub & Bidlot et al. $(2007)$ & No & UQ \\
\hline
\end{tabular}

\subsubsection{Verification Method}

\section{Buoy Verifications}

Buoy verifications are performed at a number of buoy locations around the Australian coastline. The global model examined here, at $1^{\circ}$ resolution, would not be expected to produce realistic values around complex coastlines. Hence, only buoys located in water deeper than $40 \mathrm{~m}$ are used here. Additionally, in locations where the nearest model grid point was significantly offshore from the buoy location, the buoy was not used. The locations of the buoys used for verification of the model runs in this work are shown in Figure 4.3 and further details given in Appendix C. For the purposes of reporting here, statistics are divided into groups of buoys as indicated in Figure 4.3. These groups are based on geographical location, and the type of waves likely to be encountered in each region. Buoys 56004, 56005 and 56006 are grouped with 55040 and 55026 for example, as these regions all encounter waves coming directly off the strong winds and long fetches of the Southern Ocean. Statistics are calculated and reported separately for each of these buoy groups.

Some simple quality control checks are carried out on the buoy data prior to use: non-physical values are removed, a check for faulty instruments removes more than four consecutive observations of the same value, and a check for consistency with neighbouring observations is performed. The data is generally of high quality, and the quality control required is minimal. An example is shown in Figure 4.4 for the Crowdy Head buoy (55019) for the month of July 2008.

Observations of $H_{s}$ are available from some of the buoys at half-hourly intervals (55040 and 55026), from some of the buoys at hourly intervals (55018, 55019 , and 55022) and from the remainder at 3-hourly intervals. The 3-hourly 


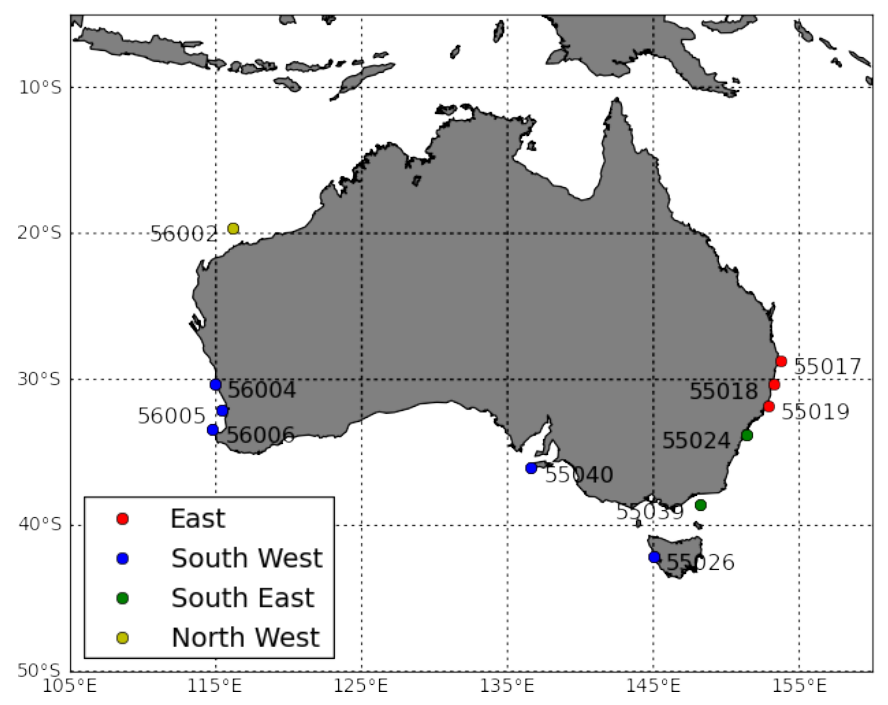

Figure 4.3: Australian buoys used in this study. Buoy groups mentioned in the verification statistics are indicated.

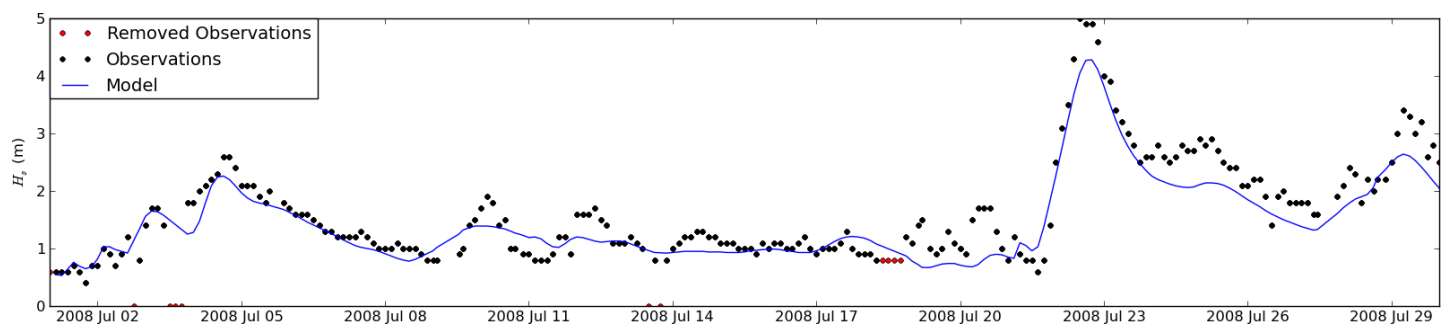

Figure 4.4: Crowdy Head (55019) $H_{s}$ buoy data for the month of July 2008, with observations removed by automatic quality control shown in red. 
model time series is linearly interpolated to the observation times. In the spatial domain, if the four surrounding model grid points are sea points, the model is bi-linear interpolated to the buoy location. If one of these points is a land point, the nearest model grid point used.

These buoys provide important data for model validation. Their position on the Australian coast, and the fact that they provide continuous time series make them a valuable source for evaluating the model in the regions where it is largely utilised, i.e. around the Australian coast. In addition to $H_{s}$, buoys also provide a means of verifying model $T_{p}$.

\section{Altimeter Verifications}

As previously discussed, altimeters provide an valuable source of data for operational wave modelling, both for data assimilation and verification. The two altimeters used here are the Poseiden-2 altimeter onboard Jason-1 (Menard et al., 2003; Carayon et al., 2003) and the RA-2 altimeter of Envisat (Resti et al., 1999), validated in Chapter 2, and corrected according to the determined corrections. As discussed previously, the inclination angles of these satellites are $66^{\circ}$ and $98^{\circ}$ for Jason-1 and Envisat respectively, the combination of the two providing good spatial coverage of the world's oceans in combination. Typical observations during a $24 \mathrm{~h}$ period for both altimeters are shown in Figure 2.1.

Altimeter data contain erroneous observations that must be removed. There are a number of means of performing this quality control. For example, prior to assimilation of this data in AUSWAM, the method of Young and Vledder (1993) was used, consisting of an initial check for gross error against the first guess model field, followed by a cross validation check for consistency with other nearby data. This serves to remove erroneous data, with the comparison with the first guess field also limiting shocks to the model. For the purpose of validation however, quality control should be done independently of the model. Here, a check is performed based on the standard deviation of the $20 \mathrm{~Hz}$ and $10 \mathrm{~Hz} H_{s}$ values for Jason-1 and Envisat respectively following Mackay et al. (2008), and nearest neighbour comparisons are performed to remove any remaining obvious errors. In order to match the spatial scales of variability between model and observations, 'super-obs' are then calculated by performing $1^{\circ}$ along track averages, consisting of 15-20 individual observations (e.g. Tolman et al., 2002; Janssen, 2008). This is demonstrated in Figure 4.5. 


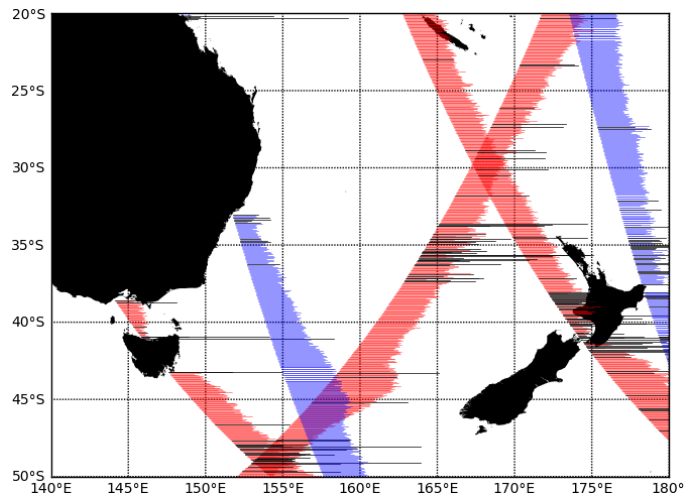

(a) Raw data

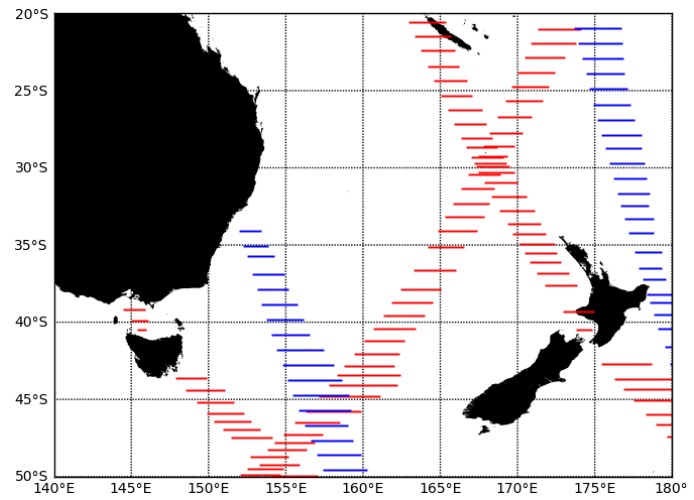

(b) Super-obbed

Figure 4.5: Jason-1 (red) and Envisat (blue) data over the Tasman Sea for $10^{\text {th }}$ July 2008. (a) shows all data with those removed through the quality control checks shown in black, and (b) super-obs calculated from the remaining data.

In general, wave model verification relies on the interpolation of the model data to the observation location to obtain a set of co-locations. Here, model data is interpolated to match the time and location of the altimeter observation by a cubic spline method to make up a set of co-locations, from which statistics are calculated. Over the July - November 2008, period examined here, this analysis resulted in more than 580,000 co-locations. Calculating statistics based on these co-locations gives an overall description of the error.

To determine the spatial variation in error, as with the $U_{10}$ verification carried out in the previous chapter, co-locations are binned into latitude-longitude bins, and statistics are calculated for each bin separately. When choosing an appropriate latitude/longitude box size, a balance must be struck between resolution and the robustness of the resulting statistics for each box due to increased number of observations. As both of these altimeters are nadir looking instruments, the total number of observations is far less than that of the swath QuickSCAT data used for wind verifications. $3^{\circ}$ was found to be a good compromise here, less than the $1^{\circ}$ achievable for the winds. It is also worth noting that the physical size of a $3^{\circ}$ box reduces at higher latitudes. However, due to the orbital characteristics of the satellite, the density of the observations also increases at higher latitudes, maintaining sufficient observations in these smaller boxes.

The total number of co-locations in each box over the four month period is shown in Figure 4.6. There are reduced numbers of co-locations along coastlines and around islands for obvious reasons. Sea ice around Antarctica results in no 
co-locations around this continent, with the shifting ice edge over this period producing in a gradual reduction in the number of co-locations around the edge. The maximum seen between $60^{\circ}$ and $65^{\circ}$ is due to the orbital characteristics of the altimeters. Over most of the globe there are around 150 co-locations for each $3^{\circ} \times 3^{\circ}$ bin. The triangular maximum in the Pacific is due to an uneven distribution of Envisat data. Envisat NRT data is processed at one of two ESA acquisition stations, with the Kiruna (Sweden) station processing 8 orbits per day and ESRIN (Italy) station processing 6. Unfortunately, operational data was not available from the ESRIN station over the period examined here, and only those from the Kiruna station are used, producing this asymmetry.

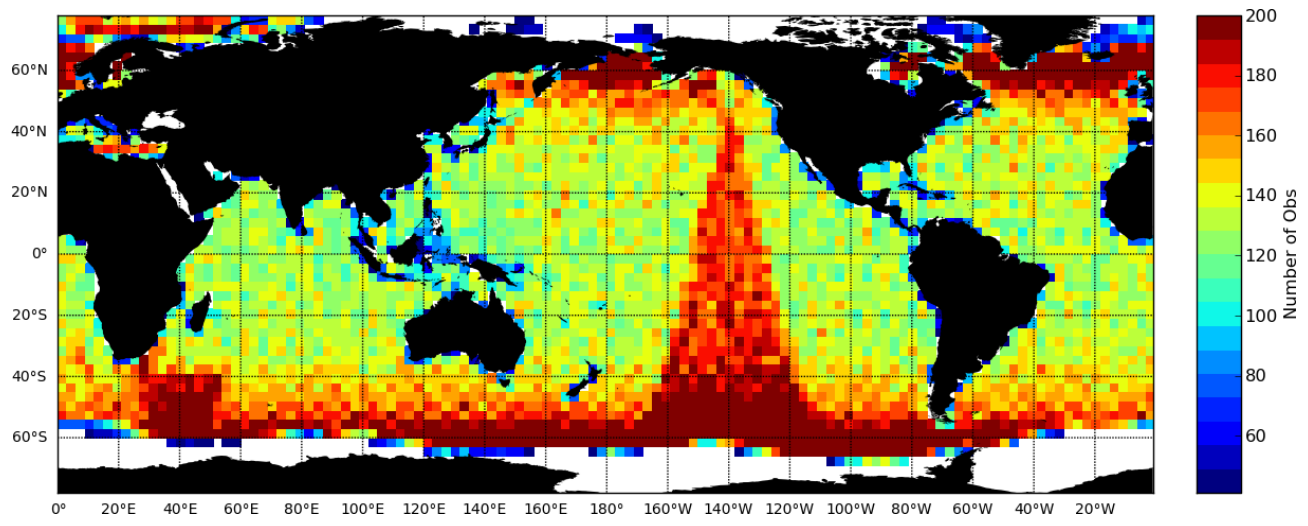

Figure 4.6: Number of altimeter-model $H_{s}$ co-locations in each $3 \times 3^{\circ}$ bin.

Statistics referred to throughout this chapter are the bias, the slope of the regression line through the origin, the RMSE and the SI (standard deviation, divided by the mean observed value) as previously defined. Each of these measures has strengths and weaknesses. We focus on the bias and the SI which gives an indication of typical scatter around this bias. It is worth noting that, as with the wind verifications, in areas of persistently low wave heights, the SI can give high values, even though the RMSE associated with these errors is small. Similarly, in such areas, small absolute biases, can be associated with large relative biases (as indicated by the slope). In instances where presenting all these statistics is impractical, relevant commentary is made in the text. 


\subsection{Results}

Results are presented here as follows: The choice of source term options is discussed in Section 4.4.1, the impact of the numerical scheme is discussed in Section 4.4.2, and the broadscale effect of sub-grid-scale blocking is presented in Section 4.4.3. To provide some context for spatial error plots, Figure 4.7 shows the $H_{s}$ (a) mean and (b) standard deviations over the globe calculated from all altimeter observations over this period, similar to those for wind speed in Figure 3.6.

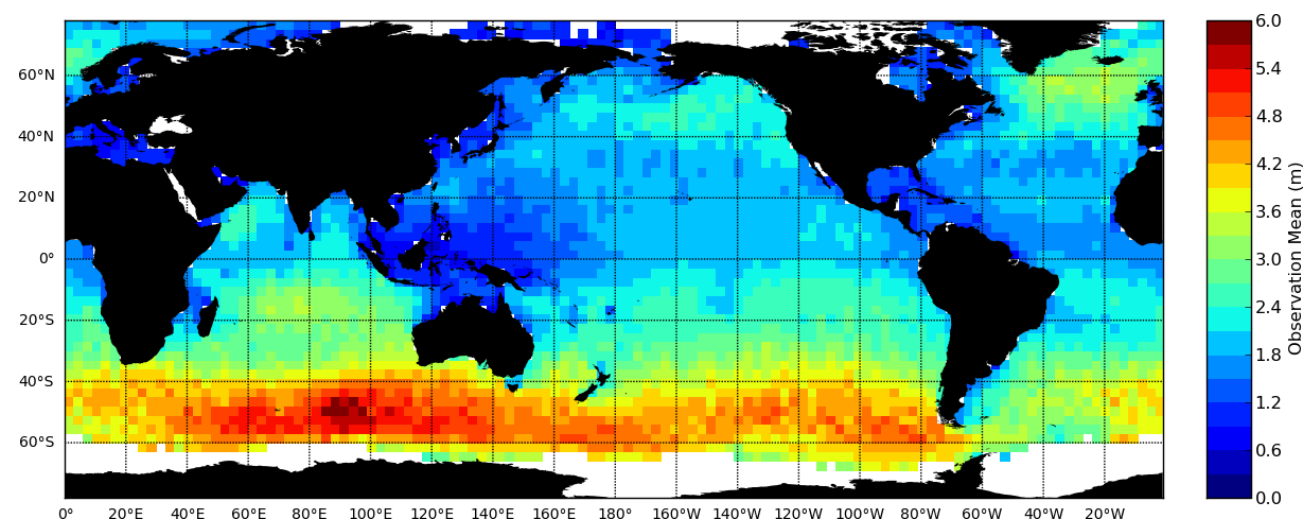

(a) Observed mean $H_{s}(\mathrm{~m})$

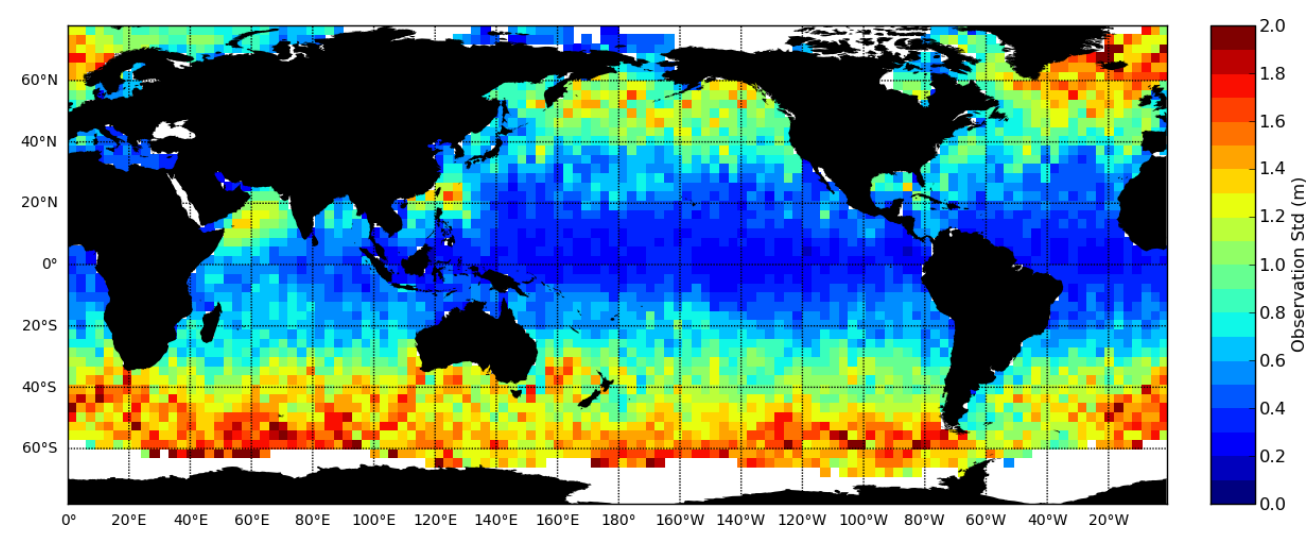

(b) Observed $H_{s}$ standard deviation (m)

Figure 4.7: $H_{s}$ statistics based on altimeter data for the period July to October 2008.

\subsubsection{Source Terms}

The original release of the WW3 employed the source term package of Tolman and Chalikov (1996) consisting of the input source term of Chalikov and Belevich (1993) and Chalikov (1995) and two dissipation constituents. The model also 
includes the option to use WAM3 source terms (Snyder et al., 1981; Komen et al., 1984) within the WW3 model framework. In the most recent release (version 3.14), WAM4 source terms (Janssen, 1991; Janssen et al., 1994) have also been added. The default implementation is an attempt to match the recent alterations of Bidlot et al. (2007, hereafter BAJ).

This section presents an analysis of model runs performed using these three source term packages (see Table 4.1). Historically, the comparison of TC96 and WAM source term variants has been hampered by the fact that they could only be used within WW3 and WAM respectively, introducing the added complication of having to account for additional differences in the models themselves (such as numerics, sub-grid-scale blocking, shallow water effects etc.). The ability to run all these source term packages from within the same model removes this complication. As the development of both TC96 and BAJ terms were motivated by the desire to correct known deficiencies in the WAM3 terms, they can be expected to produce improved results. WAM3 terms are included here simply for completeness.

The aim is to determine, in a fairly broad sense, the error characteristics associated with each source term option when forced with ACCESS winds. The focus here is not the attribution of forcing/wave model error. This is addressed in more detail in Chapter 6. However, knowledge of the error characteristics of the forcing winds, presented in Chapter 3 provides an important context to the discussion.

A summary of the $H_{s}$ verification statistics for the three source term packages is shown in Figure 4.8 for both buoys and altimeters. Focusing initially on the altimeter results, all three show negative bias, with WAM3 showing the most by a significant margin, and TC96 showing the least. Overall, the RMSE is comparable for both BAJ and TC96, with the negative bias in both being slightly greater for BAJ, and the SI of BAJ being marginally better than that of TC96. Given the negative bias in the winds over much of the globe (e.g. Figure 3.7), a negative bias in the waves is not a surprising result. This is addressed in more detail in Chapter 6. For the purposes of this discussion, it is sufficient here to acknowledge that this common negative bias is largely the result of a negative bias in the forcing winds, and it is in this context that the comparison of source terms below is made.

Examining these error statistics in a spatial context, Figure 4.9 shows bias and SI over the globe for each of these source terms. Though clear differences 


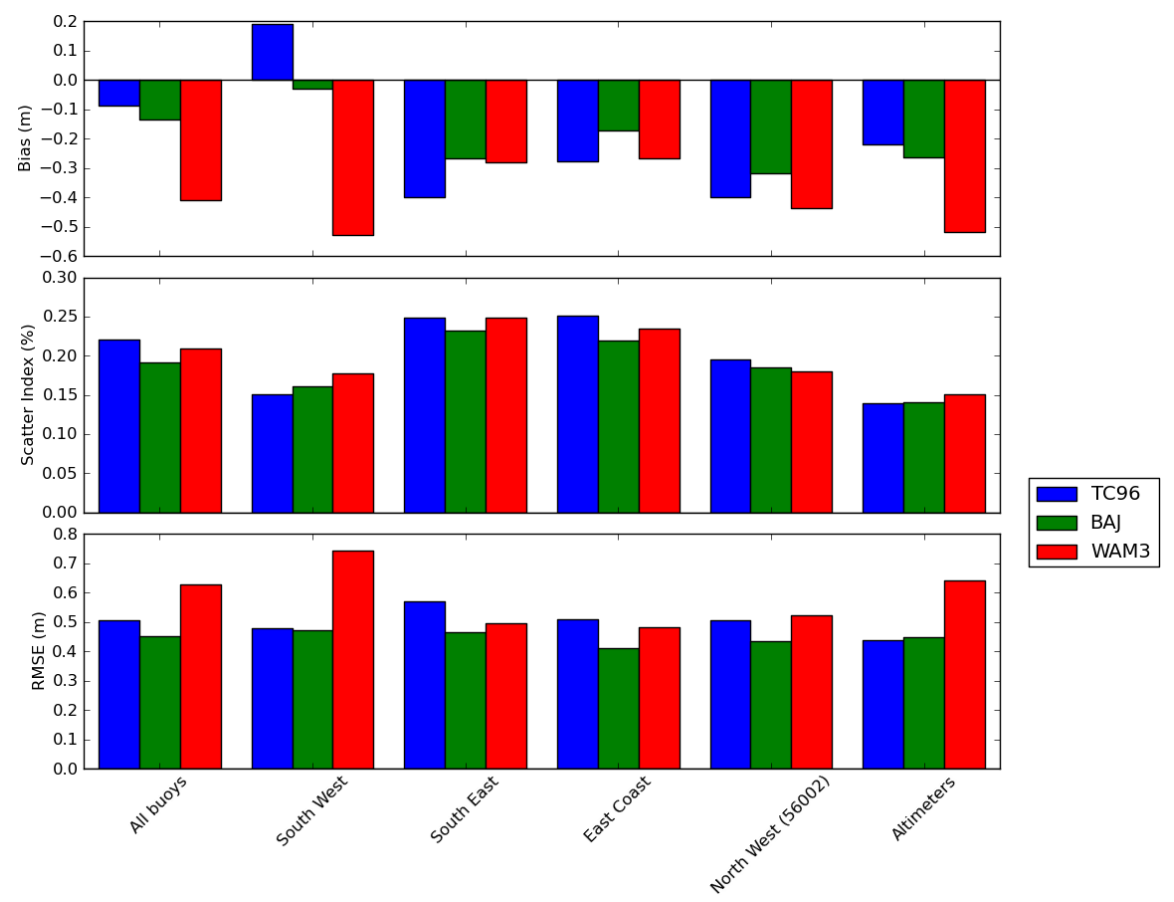

Figure 4.8: Overall $H_{s}$ statistics for BAJ, WAM3 and TC96 source term packages relative to buoys shown in figure 4.3 and global altimeter data.

exist, the negative bias in $H_{s}$ is present for all source term options over much of the globe. WAM3 shows a more severe negative bias than both BAJ and TC96, particularly in the Southern Ocean. This negative bias in the WAM3 formulations has been noted by several other studies (e.g. Lionello et al., 1992; Bender and Glowacki, 1996; Rogers, 2002). BAJ and TC96 also produce much better results than WAM3 in terms of SI over the whole globe. Given the clear superiority of these terms, the following discussion focusses only on these two source term packages.

There are notable features common to both BAJ and TC96 error characteristics. Both show high SI to the north of Indonesia. These are primarily due to low wave heights in this region. Absolute values in terms of RMSE are less severe (not shown). The negative bias in this region is of note in the context of the wave climate, with slope indicating strong underestimations (not shown). Though the models (both atmospheric and wave) can be expected to show relatively large variable error in these areas of low wind speed and wave height, data quality is also likely a contributer, with increased relative scatter expected.

High SI can be seen around coastlines. There are a number of factors likely to be contributing to this error such as errors associated with poorly resolved 


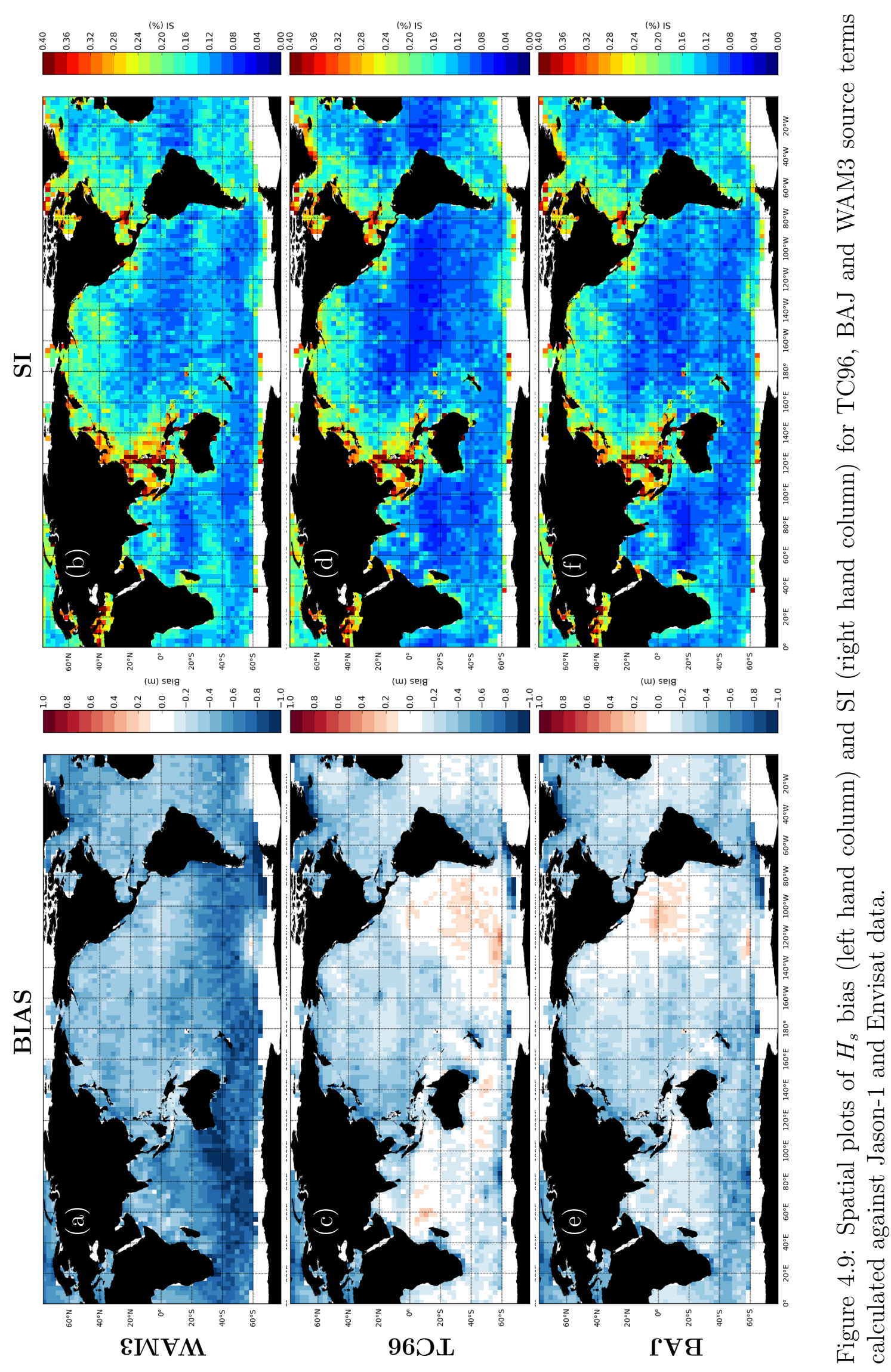


coastal processes within the wave model, ocean surface current systems and error associated with the inaccurate wind forcing. At this resolution, little can be concluded about the relative role of shallow water effects. It is apparent that in the south of the African, South American and Australian continents, bias and SI are worse on the east coasts. This is consistent with wind error being the major cause of error in these predominantly young, fetch limited wave conditions. Indeed, a negative bias in the wind speed was identified on these coasts in Chapter 3 , which is likely producing biases as well as variable error that is imparted onto the wave field.

Over much of the Northern Hemisphere, biases are also qualitatively similar, with both BAJ and TC96 showing reasonably consistent negative biases of around 20-40 cm. Significant differences are apparent in the Southern Hemisphere however, with BAJ terms indicating strong negative biases, while TC96 shows some areas of slight negative bias as well as positive biases to the south of Australia and the South Pacific. This bias for TC96 in the southern mid-latitudes is notable due to the fact that the winds are known to be negatively biased in this region (Figure 3.7). This suggests that with unbiased winds, TC96 would produce waves that are too energetic in these generation areas of the Southern Ocean. This is supported by ongoing operational verifications performed at the Bureau. The Bureau currently receives operational wave forecasts from both the National Oceanic and Atmospheric Assciation (NOAA) and UKMO wave forecast systems. Both of these systems are based on recent versions of WW3, both using TC96 source terms. Ongoing monthly verifications of both wind and waves from these models (Bidlot et al., 2002, ${ }^{2}$ ), show that both of these centres have winds that do not show the degree of negative bias that ACCESS does. From operational buoy verifications, and through anecdotal evidence from forecasters, these models consistently show a significant positive bias on the Australian west coast.

The positive bias produced by TC96 terms the in South Pacific extends into the Tropical Eastern Pacific as this over-predicted wave energy propagates. For BAJ terms, despite a negative bias in the generation areas of the southern midlatitudes, a strong local positive bias is present in the Tropical Eastern Pacific. This is due primarily to a lack of swell dissipation in WAM variant source terms. Observations of swell dissipation are consistent with the effect of friction at the

\footnotetext{
${ }^{2}$ http://www.jcomm.info/index.php?option=com_content\&task=view\&id=107
} 
air-sea interface, resulting in a flux of momentum from the wave field to the wind (Collard et al., 2008). This flux is entirely absent from WAM source term variants. TC96 this includes swell attenuation, in the form of a negative wind input for waves travelling faster than, or at large angles to the wind (Chalikov and Belevich, 1993). Notably smaller SI in the Tropical Pacific for TC96 than for BAJ can likely be attributed to the inclusion of this effect in the former. The contribution of swell dissipation to the overall wave spectral balance has been added to the WAM source terms in the recent formulations of Ardhuin et al. (2010), but these are not tested here.

In general, buoy results are consistent with those of the altimeter comparisons (Figure 4.8). For SI, BAJ outperforms TC96 at all regions except the South West. TC96 shows the lowest bias when averaged across all regions, however BAJ shows more consistency across buoy locations. The magnitude of the bias for each region is smaller for BAJ, and is negative everywhere, while TC96 produces a positive bias for the South West buoys and a negative bias at all other locations. This is consistent with TC96 inherently over predicting in the long Southern Ocean fetches within a general state of negative bias due to the forcing winds.

$T_{p}$ verifications are also shown in Figure 4.10 (note that there are no $T_{p}$ statistics for altimeter data). Overall, WAM3 shows a clear negative bias. TC96 shows a slight negative bias with BAJ indicating negligible biases. In terms of SI, results are comparable for TC96 and BAJ, both showing gains over WAM3. Overall, it is difficult to draw any definitive conclusions in regards to $T_{p}$.

To summarise, all source terms are found to produce an overall negative bias in $H_{s}$ results, consistent with the identified negative bias in the ACCESS forcing winds. TC96 and BAJ terms are clearly superior to the WAM3 terms. Northern Hemisphere biases are comparable for TC96 and BAJ. In the Southern Hemisphere, BAJ shows a strong negative bias, while TC96 terms produce slight positive biases to the south of Australia and in the South Pacific. This is suggestive of the fact that TC96 terms inherently produce overestimated $H_{s}$ in the Southern Ocean, which are somewhat mitigated here by a negatively biased winds. In the context of the forcing, BAJ terms are found to produce a more consistent negative bias over the globe, with the exception of the eastern Tropical Pacific, due to a lack swell attenuation. These features are discussed further in Chapter 6. 


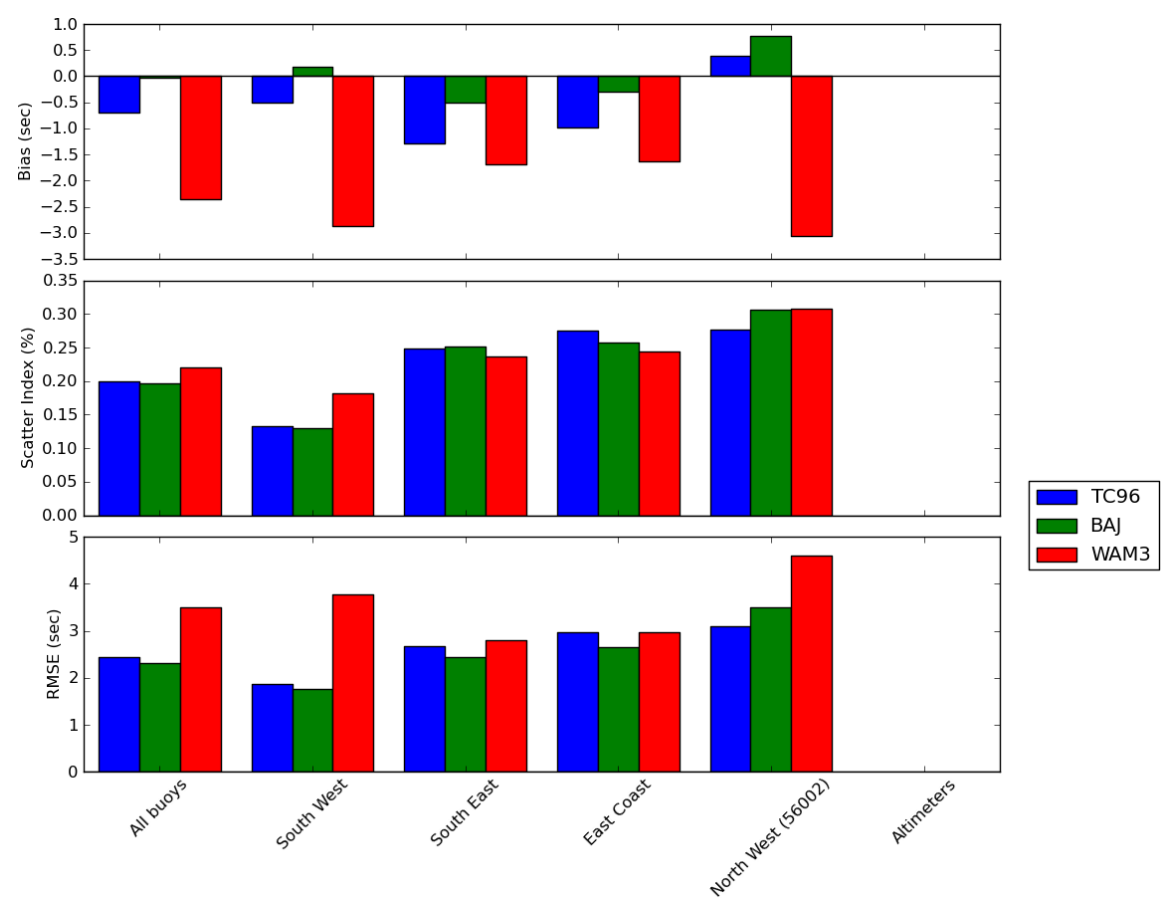

Figure 4.10: Bias and SI for $T_{p}$ for different source term options.

\subsubsection{Numerics}

The default numerical scheme in WW3 is the UQ scheme discussed above. The model also includes a first order scheme. The third order scheme is generally considered superior, with the latter being included mainly for testing purposes. However, despite the obvious attraction of higher order schemes, some contention remains as to their benefit in the context of wave modelling. Though it is easily demonstrated that a first order scheme can not adequately advect a spike of energy, in real world wave modelling applications, the structure of the wave field is rarely this extreme. It has been suggested that, at least in the case of integrated parameters, there is little to be gained from these higher order schemes (Rogers et al., 2005, WISE Group, 2007).

The most recent implementation of AUSWAM at the Bureau used the third order upwinding scheme of Bender (1996). From verifications of a one month hindcast using three buoys located around the Australian coast, it was concluded that the first order upwinding propagation numerics of WAM were responsible for excessive dissipation of wave energy, particularly swell, causing negative biases in $H_{s}$ over much of the Australian coastline. This has subsequently been refuted by Wittmann and O'Reilly (1998) and Rogers (2002), who showed with the use of a 
great circle ray tracing tool, that the first order numerical scheme of WAM is unlikely to be a primary source of negative bias in the model operated at FNMOC. Rogers et al. (2005) also make the point that all the numerical schemes used in these models are mass conserving, stating that while they be may indirectly responsible for local biases in conjunction with island blocking for example, overall, they do not dissipate energy, and cannot be responsible for overall biases. It is noted here, however, that conservation of energy does not ensure conservations of $H_{s}$ (with $H_{s} \propto \sqrt{E}$, from equation 4.8).

To examine the influence of numerics, model runs using both the first order and the UQ scheme were performed. Runs are otherwise identical, both employing BAJ source terms. Figure 4.11 shows time series of both $H_{s}$ and $T_{p}$ for both numerical schemes at the Cape du Couedic waverider buoy (shown in Figure 4.3, buoy 55040). Some smoothing is visible, though it does not appear to be a large effect. It should be noted however, that given the location of this buoy on the southern Australian coast, this time series is likely dominated by wind sea, and smoothing could be expected to be more significant for swell systems.

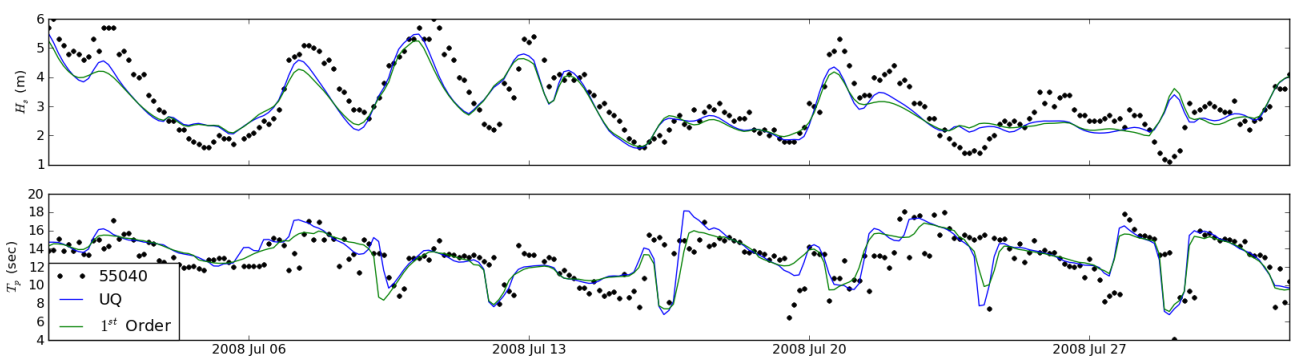

Figure 4.11: Time series of $H_{s}$ (a) and $T_{p}$ (b) for both the UQ and $1_{s t}$ order numerical schemes at the Cape du Couedic waverider buoy for the month of July 2008.

Statistics for these runs based on buoy and altimeter verifications are shown in Figure 4.12. These show an increased negative bias in the UQ results, contrary to the assertions of Bender (1996). This increase is consistent across all buoys, and is more pronounced than that seen for altimeter comparisons, suggesting that the choice of numerical scheme is having a bigger impact at the coast than in the open ocean. In terms of SI, though differences are small, again they are more pronounced in the buoy data.

This can be clearly seen by examining the difference between the model $H_{s}$ means for each scheme, shown in Figure 4.13. Locally differing bias characteristics are visible in the lee of islands. Both schemes are expected to produce unrealis- 


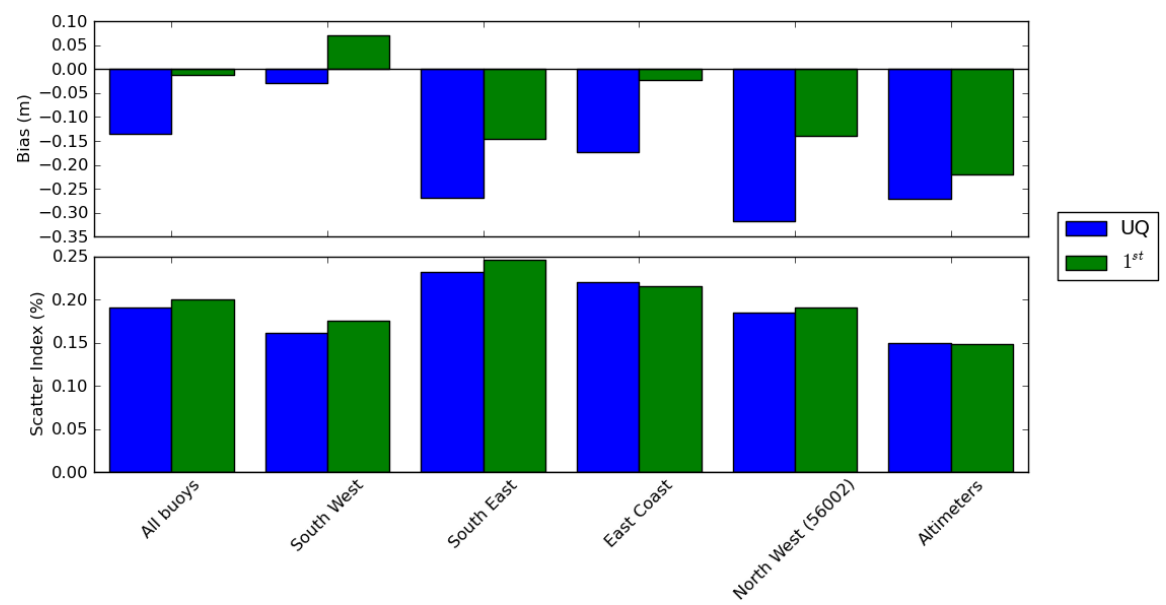

Figure 4.12: Statistics for 1st order and UQ numerical schemes relative to buoy and altimeter data.

tically smooth shadow zones in island wakes, though for different reasons. In the case of the first order scheme, this is due to lateral numerical diffusion. For the UQ numerics, Tolman (1995) demonstrate that the use of the, in their case Booij and Holthuijsen (1987), diffusive GSE correction in the UQ produces the same defect. Similar results are shown for the averaging GSE alleviation, used here, by Tolman (2002b). Quantitative differences in the severity of this deficiency may locally account for some of these differences. (Note that an alternative method is proposed by Tolman (2002b), in which divergence is added to the advection field, with this technique noted by the author as potentially allowing for GSE alleviation without removing shadow zones behind islands. However, this is not included in the current version of WW3 due to a lack of maturity). The bulk of this difference is likely due to influence of boundary conditions. The mean $H_{s}$ is noticeably lower for UQ results for waves travelling perpendicular to boundaries, most notably along the imposed boundary condition of the Antarctic ice edge for example. Local propagation differences result in differing amounts of wave energy being removed from the system as it encounters these boundaries. It can be demonstrated from simple propagation tests along slanting coastlines that the first order scheme does indeed lose more energy than the UQ scheme.

These differences in mean $H_{s}$ are consistent with validation results. Large differences can be seen in the wake of Tasmania with UQ numerics producing lower mean $H_{s}$ values than the first order scheme. This can be seen in the relative biases for the south east and east coast buoys (Figure 4.12). Though $H_{s}$ results are degraded here by the use of UQ numerics, it is stressed that this case does not 


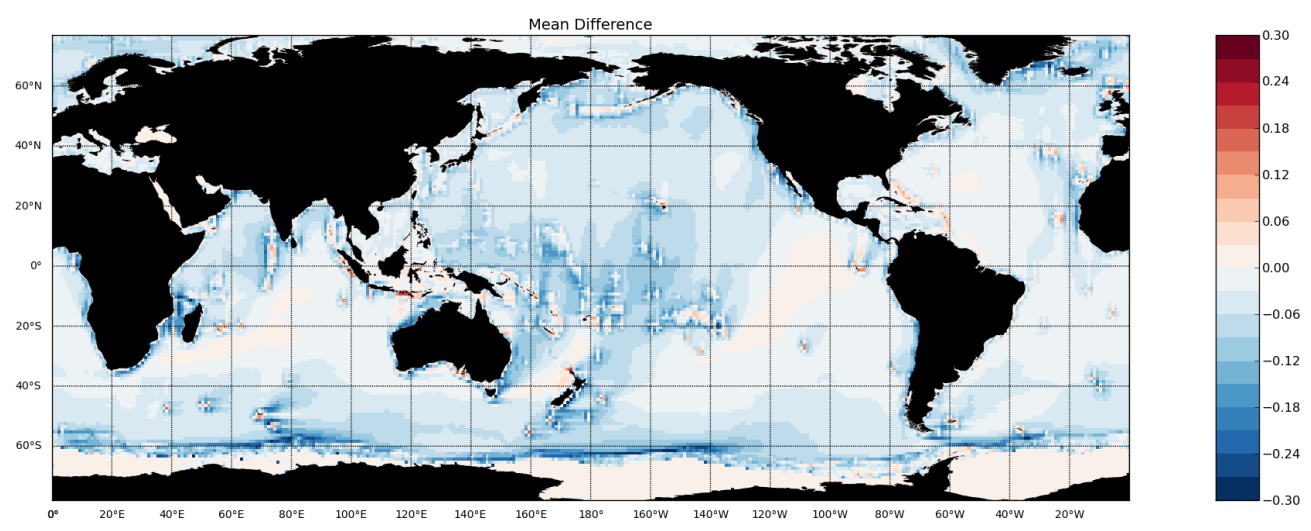

Figure 4.13: Mean difference $(\mathrm{m})$ between $H_{s}$ from the 1st order and UQ numerical schemes (UQ - $1_{s t}$ ).

provide reliable assessment of the relative merits of these schemes. The existence of a (likely) wind related negative $H_{s}$ bias in this region has been previously discussed. The fact that this is reduced by the use of first order numerics may simply imply some compensation of opposing systematic error (existing negative bias compensated by positive bias due to the first order scheme).

Despite altimeter verifications indicating similar values of SI for both numerical schemes for the global data set, significant spatial variation is apparent. Figure 4.14 shows the spatial distribution of the percentage improvement in SI for UQ over the first order scheme, with improvements evident over much of the globe, with the exception of the eastern Tropical Pacific. At first glance, this is a surprise given the predominance of swell in this region. However, the lack of swell attenuation in the BAJ source terms results in too much swell in this region (as discussed above), and the UQ scheme is most likely simply correctly propagating these deficiencies.

It is also worth making the point here, that applying point based verifications to a spatial forecast, may favour the 1st order scheme in terms of these verification statistics. In addition to error in the intensity of a storm, there is also a phase error, or location error. If a storm's intensity is predicted correctly, but it is misplaced in space or time, comparisons with a passing altimeter track will result in a large error both at the location of the model maximum, and its true location. A numerical scheme that smooths out the field, will record a lower error in both locations, so while a correctly placed storm verifies better in the UQ scheme, an incorrectly placed storm verifies better for the more diffusive $1^{\text {st }}$ order scheme. In many applications, a storm whose intensity is correctly forecast but slightly 


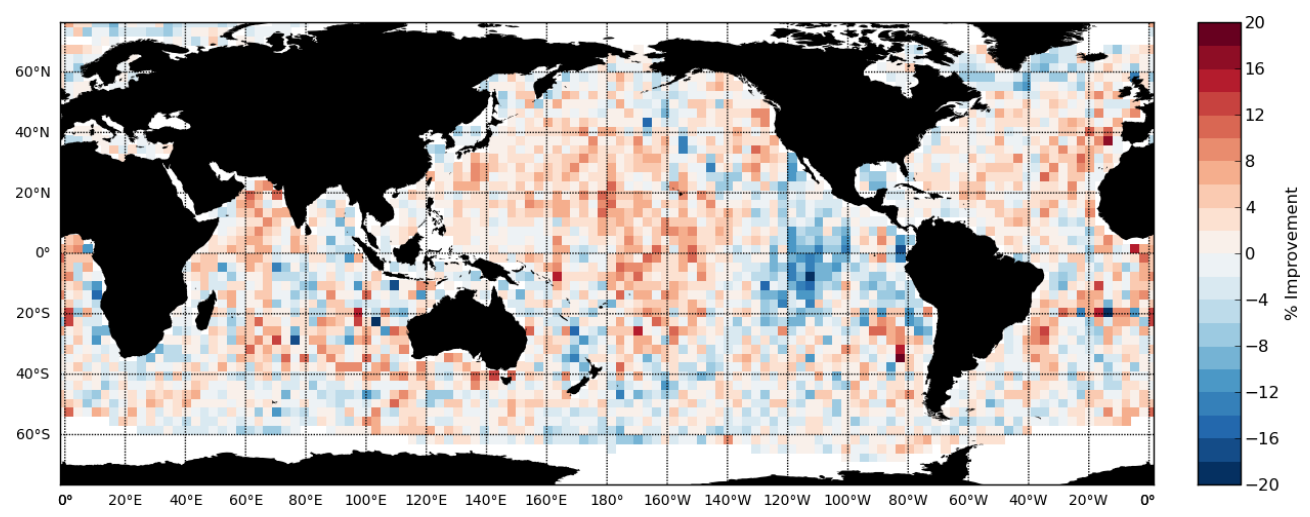

Figure 4.14: Percentage improvement in $H_{s}$ SI for UQ over the first order scheme $\left(\left(S I_{1 s t}-S I_{U Q}\right) / S I_{1 s t} \times 100\right)$.

mis-placed is more desirable than one whose intensity is under-predicted. This is not necessarily reflected in these verification statistics.

Despite inconclusive results for the use of the third order scheme, we tend to agree with the conclusions reached by the WISE Group (2007), that is, that if methods are available to compute propagation more accurately without a large increase in computation time, then these methods should be used. Examining overall error statistics does not provide an effective means of assessing numerical schemes, with the observed model error being a complex amalgamation of many imperfections. Idealised tests clearly demonstrate the superiority of the UQ scheme in more closely describing the analytical solutions (Tolman, 1995). The fact that the first order scheme masks the problem of an existing negative bias is not an argument for its use. In the context of the overall goals of this thesis, the aim here is to minimise sources of large scale error in the wave model, in order to assess the contribution of systematic wind error, and the superposition of opposing error sources is not a desired outcome. Hence, the UQ scheme is used for the remainder of this work.

\subsubsection{Sub-Grid-Scale Blocking}

The following section examines the influence of sub-grid-scale blocking on the large scale error. The benefits of the blocking of wave energy by islands that are too small to be explicitly resolved are clearly outlined in Tolman (2003). Some qualitative comparisons is made here between runs performed with and without sub-grid-scale blocking active. These simply serve the purpose of illustrating the 
importance of the inclusion of these effects in the context of large scale error attribution examined in this thesis. Runs examined here use BAJ source terms, and UQ numerics, with and without sub-grid-scale blocking activated.

An example of the effect of sub-grid-scale blocking on the propagation of a swell field through French Polynesia is shown in Figure 4.15. In the absence of such blocking, more wave energy is passing through the island chain, reducing $H_{s}$ both here and in the island wakes. This has significant impact on the large scale. From the spatial bias and SI plots shown in Figure 4.16, the impact can be seen to extend far beyond the location of the obstruction. In this particular case, the effect of unblocked swell incorrectly propagating from the Southern Ocean generation regions into the Pacific is evident throughout the Tropical Pacific and extending up into the North Pacific.

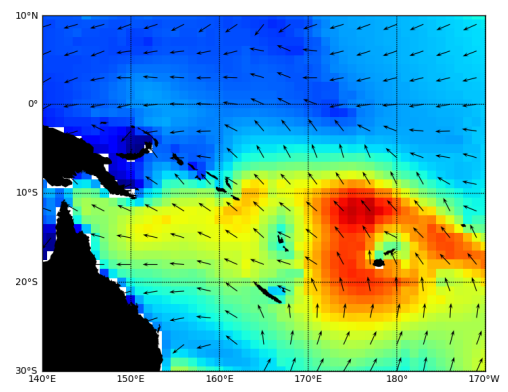

(a) Sub-grid-scale blocking

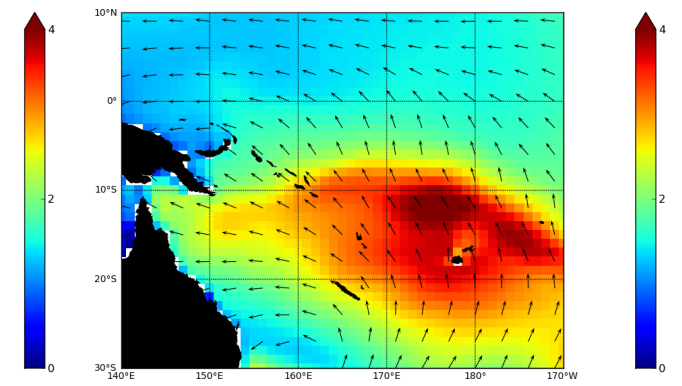

(b) No sub-grid-scale blocking

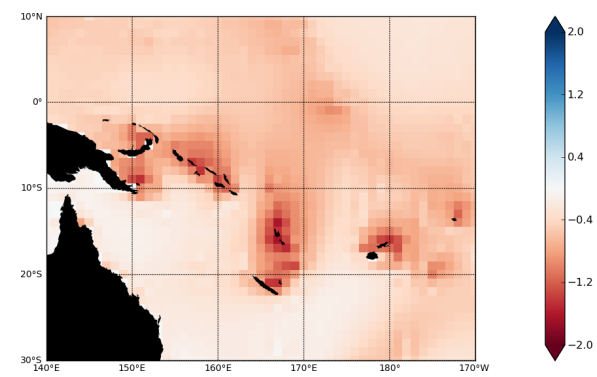

(c) Difference ((b)-(a))

Figure 4.15: $H_{s}(\mathrm{~m})$ snapshots for a wave system propagating northward through French Polynesia using BAJ source terms and UQ numerics with (a) and without (b) sub-grid-scale blocking activated and the difference between the two (c).

In general, the importance of this sub-grid-scale blocking parameterisation on global $H_{s}$ biases is clear. These are included in all remaining hindcasts discussed in this thesis. 


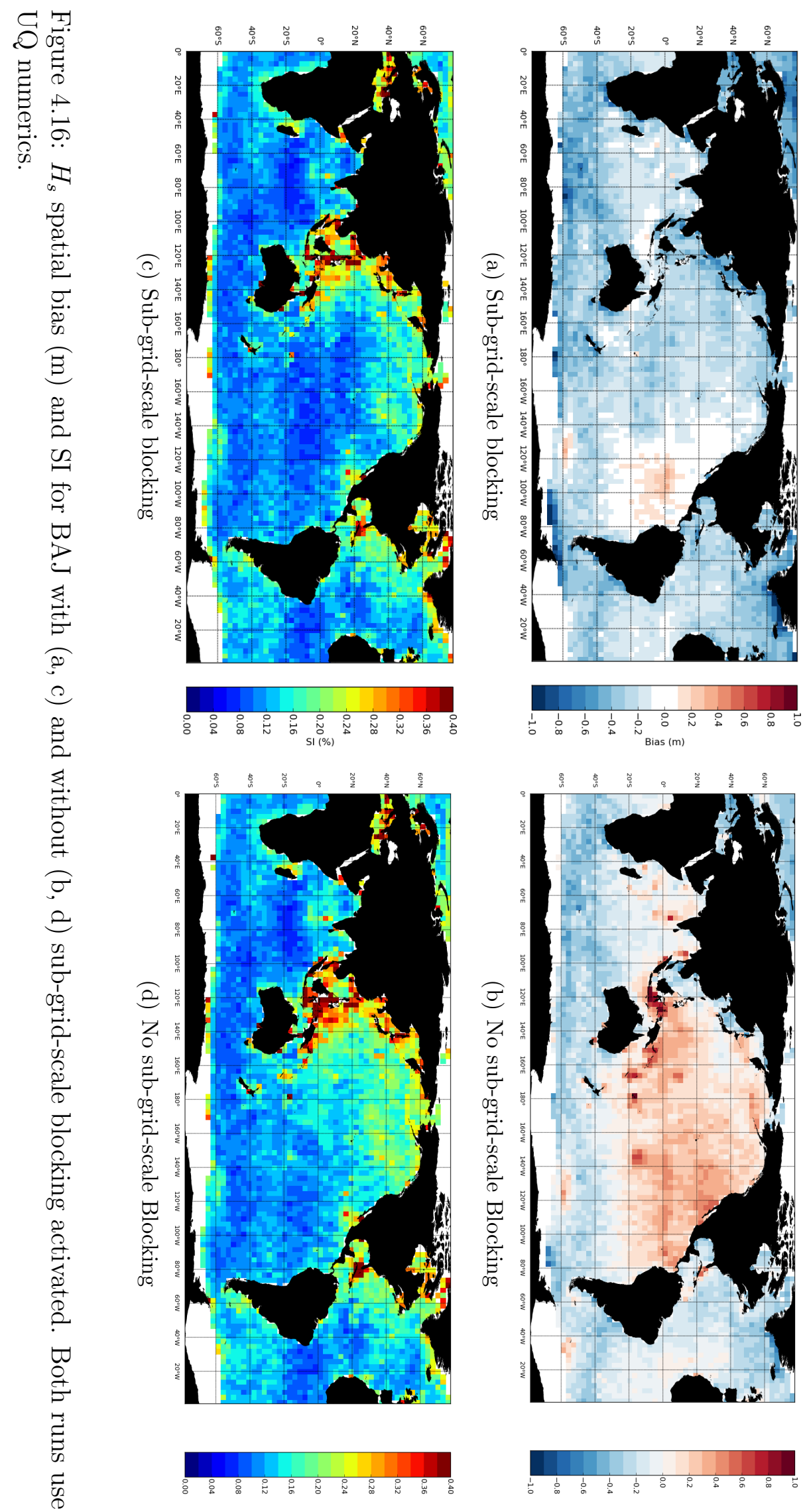




\subsection{Summary}

This chapter has documented the set up and verification of the WW3 under ACCESS forcing. Evaluation of a number of hindcasts has been carried out, examining the impact of choices of source terms and numerics. A negative bias in $H_{s}$ is present in all hindcasts. This is attributed mainly to the negative bias in the ACCESS forcing winds, identified in Chapter 3. In this context, TC96 was found to over-predict in the long fetches of the Southern Ocean, resulting in over-predicted $H_{s}$ values on the Australian west coast. BAJ terms were found to produce a more consistent negative bias over the globe, with the exception of the eastern Tropical Pacific, due to a lack of swell attenuation within these terms. The UQ numerical scheme was found to produce slightly better SI values than the $1^{\text {st }}$ order scheme. Lower mean $H_{s}$ in island wakes for the former also resulted in slighter lower overall biases from the UQ scheme.

In the context of the overall aims of this thesis, the primary role of systematic error in the ACCESS forcing in the overall negative $H_{s}$ bias is clear. A number of wind correction methods are explored in the following chapter (Chapter 5). The contribution of wind error to the wave field, as well as the effects of these corrections, are then discussed in Chapter 6. 


\section{Chapter 5}

\section{Statistical Wind Corrections}

\section{Contents}

5.1 Introduction . . . . . . . . . . . . 81

5.2 Background .................. 82

5.3 Method ..................... 85

5.4 Results .................... 87

5.4.1 Static and Homogeneous . . . . . . . . . . . . 87

5.4 .2 Spatially Varying Corrections . . . . . . . . . . . 88

5.4.3 Spatially and Temporally Varying Learned Corrections 95

5.5 Discussion ................. 106

5.6 Conclusions . . . . . . . . . . . . . 109

\subsection{Introduction}

In Chapter 3 a low bias in the ACCESS surface winds was identified. WW3 runs forced with these winds were found to similarly produce negatively bias $H_{s}$ results in Chapter 4. The overall aims of this thesis include exploring the degree to which statistical corrections to the forcing winds can address systematic bias in the wave field. This chapter will focus on statistical means of removing the biases in the forcing winds. The effect of these corrections on the wave field are then assessed in Chapter 6. 
A number of methods of statistically correcting the wind speed are examined. The simplest of these is a homogeneous correction applied over the entire global domain, calculated from comparisons against QuikSCAT scatterometer observations. This is then extended to a correction that varies in space, calculated independently for each model grid point, retrospectively for the entire period. Finally, a learned correction technique is proposed, in which spatially varying corrections are determined from comparisons against scatterometer observations from preceding forecasts. The use of past observations to determine corrections in this way results in a continually evolving correction that adapts to changes in the bias characteristics of the model, thus removing the need to monitor and manually update corrections as these change.

Previous work examining statistical corrections to surface winds is summarised in Section 5.2. The methods for wind corrections are then outlined in Section 5.3. Results are presented in Section 5.4 and some conclusions drawn in Section 5.6.

\section{$5.2 \quad$ Background}

Where systematic errors in the wind field are known a-priori, these can be removed with statistical corrections. Tolman (1998b), for example, conducted a validation of the GFS surface winds prior to the operational implementation of WW3 at NCEP. For the Northern Hemisphere winter of 1994/1995, modelled winds were compared to buoy and ERS-2 altimeter data. Based on these findings, a wind correction was introduced, prior to tuning the wave model. This correction consisted of a coastal error correction up to $150 \mathrm{~km}$ offshore, a deepocean correction beyond $300 \mathrm{~km}$ offshore, and a smooth blending in between. The original deep water correction consisted of a substantial linear correction:

$$
U_{c}=1.10 U_{o}-1.5
$$

where $U_{c}$ and $U_{o}$ were the corrected and original wind speeds respectively. By early 1997 wind speed biases were greatly reduced, and the following error corrections were used in the initial operational implementation of WW3 (Tolman et al., 2002): 
Coastal:

$$
U_{c}=U_{o}-0.3
$$

Open Ocean:

$$
U_{c}=1.05 U_{o}-1.0
$$

Subsequent improvements in the atmospheric model in June 1998 again reduced wind speed biases, resulting in the removal of these corrections in June 1998 (Tolman et al., 2002).

Similar statistical corrections have been applied to wind fields from the $\mathrm{Bu}-$ reau's previous operational atmospheric model GASP prior to forcing the wave model AUSWAM. (Greenslade et al., 2005) removed the low bias in the GASP winds identified by Kepert et al. (2005) and Schulz et al. (2007) with the following corrections.

$$
u_{c}=1.11 u_{o}, \quad v_{c}=1.25 u_{o}
$$

This was found to have positive effects on the forecast $H_{s}$, when compared to buoy data around the Australian coast.

While such adjustments provide a useful means of removing known biases in the forcing winds, these simple corrections have a number of limitations. The first is the inability to account for the expected spatial variation in the wind error. The results presented in Chapter 3 showed that the ACCESS $U_{10}$ bias exhibits considerable variation over the global domain. This spatial variability has been similarly demonstrated by Chelton and Freilich (2005) in the case of the ECMWF and NCEP operational analyses. Applying a single, homogeneous correction to the entire field does not account for this spatial variation, and winds corrected in this way can be expected to retain significant regional biases. The verifications of the Bureau's GASP marine winds (Kepert et al., 2005; Schulz et al., 2007) did not include a spatial assessment of error, with a single correction proposed for the entire global domain. Subsequent work has demonstrated that the GASP model showed considerable regional variation, with positive biases in the tropics, and negative biases in the mid-latitudes (Durrant and Greenslade, Submitted). The corrections of Greenslade et al. (2005) above, while improving the mid-latitudes bias, likely degraded performance in the tropics. Resulting improvements seen in wave model results at mid-latitude buoys locations around Australia would be 
unlikely to occur everywhere.

Tolman (1998b) did consider the spatial variation of error, and partly accounted for this by applying separate corrections around coastlines. However, in the open ocean, the proposed correction simply prioritised bias minimisation in the Northern Hemisphere, in accordance with NCEP's forecasting priorities, actually introducing a slight positive bias in the Southern Hemisphere. A more sophisticated approach is taken by Cardone et al. (2004). In an attempt to improve the NCEP/NCAR Reanalysis winds, corrections are calculated for each model grid point based comparisons with QuikSCAT observations over a number of years. These corrections are then applied to the wind field, prior to some additional manual kinematic analysis. This technique, proves very effective at removing spatially varying biases, however, it can only be applied to hindast winds.

This leads to the second limitation. Once determined, any applied correction to an operational system will need to be continually monitored for changes in systematic wind biases with seasonal variation, and alterations and upgrades to the atmospheric model. Such changes to the forcing are an issue for any downstream model. Indeed, part of the motivation for performing wind corrections is an acknowledgement of this fact, with adjustments to statistical wind corrections, in general, being assessed as less burdensome than retuning the wave model itself. However, maintaining such wind corrections still requires considerable effort. Ideally, a correction method that can automatically adapt to these changes is required.

One such technique is the Operational Consensus Forecast (OCF) scheme of Woodcock and Engel (2005). In its complete form, OCF combines forecasts derived from a multi-model ensemble to produce an improved real-time forecast at locations where recent observations are available. Component model biases and weighting factors are derived from a training period of previous model forecasts and verifying observations. The next real-time OCF forecast is a weighted average of the set of latest-available, bias-corrected, component forecasts. Determining corrections based on a defined learning period in this way results in a correction that evolves in time with the changes in recent systematic model biases, without the need for manual monitoring.

The application of OCF forecasts to marine winds was investigated by Durrant et al. (2009b) using ten models at 14 buoy sites located around North Amer- 
ica. The focus of their study was on the performance of weighted averages of several models, which is not the aim here. However, it included what the authors refer to as 'internal methods', those in which an individual model forecast is corrected according to a training set based only on that particular model. For $U_{10}$, an average $16 \%$ improvement in individual model RMSE was achieved by applying linear corrections, based on a 30 day learning window.

This technique is constrained by the need for observations with which to 'train' the model. This has generally restricted its application to site based locations, for which consistent historical observations are available. In the case of Durrant et al. (2009b) for example, forecasts rely on buoy observations. Here, the aim is to correct the entire gridded wind field.

In the absence of a dense observing network to represent 'truth' for the purposes of training, an analysis field has been substituted in several studies as a reference to which to compare forecast products. Recent work at the Bureau has investigated the correction of gridded fields of surface temperature against the Mesoscale Surface Analysis System (MSAS) analysis (NMOC, 2008, 2010b). Inherent systematic background error can be expected to grow with forecast period, and hence the potential to benefit from analysis based corrections exists. This approach offers the advantage of a training data set with regular output at every grid point which is being corrected. However, in general, and indeed specifically in the case of surface winds examined here, while assimilation techniques have undoubtedly provided significant gains in analysis accuracy, biases remain (e.g. Chelton and Freilich, 2005). The corrected product can do no better than replicate the systematic errors of the analysis.

The method applied here aims to produce an observation based, spatially varying, learned correction scheme that can be applied to forecast winds, thus addressing both the spatial variation in systematic $U_{10}$ error, as well as removing the need to manually monitor, and update the applied correction.

\subsection{Method}

The OCF methodology of Woodcock and Engel (2005) is a simple statistical scheme, which takes a weighted average of bias-corrected component model forecasts on a site-by-site and day-by-day basis. The scheme is based upon the 
premise that each model derived forecast $\left(f_{i}\right)$ has three components: the true value $(o)$, a systematic error component or bias $\left(b_{i}\right)$ that can be approximated and removed, and a random error component $\left(e_{i}\right)$ that can be minimised through compositing ( $i$ indicating each separate model). Bias and weighting parameters are based on a moving window of historical data. Normalised weighting parameters $\left(\hat{w}_{i}\right)$ are calculated by using the inverse Mean Absolute Error (MAE) from the bias-corrected error samples of the $n$ contributing model forecasts over the training period.

The OCF scheme described above uses site based observations to determine a learned correction. The aim here is to correct the entire wind field grid, applying an independent correction for each grid point. This is achieved with the use of QuikSCAT scatterometer data. QuikSCAT swath data is binned into $1 \times 1^{\circ}$ bins, as done in Chapter 3, and independent learned corrections calculated based on the co-locations in each bin.

To achieve this end, a number of intermediate steps are carried out. Overall corrections, similar to those carried out by Tolman (1998b) and Greenslade et al. (2005), are first explored. These simple corrections primarily serve to provide a benchmark for which to assess the relative gains achieved by more complex methods. Spatially varying corrections are then examined, with independent corrections calculated for each model grid point, retrospectively for the entire period. This approach is quite similar to that taken by Cardone et al. (2004). This spatially varying correction is then extended to vary in time, based on the learned correction concept of the OCF technique above, with appropriate adjustments determined from comparisons between scatterometer observations and preceding forecasts. These correction types are referred to for the remainder of this chapter as static homogeneous, static spatially varying, and spatially and temporally varying learned corrections respectively.

Statistical corrections applied include a simple bias correction, a multiplication factor (referred to here as a slope correction), and a linear correction, i.e.:

$$
\begin{array}{cl}
\text { Bias : } & U_{c}=U+b \\
\text { Slope: } & U_{c}=s U \\
\text { Linear: } & U_{c}=l_{1} U+l_{2}
\end{array}
$$


where $U$ and $U_{c}$ are the wind speed and corrected wind speed respectively, and the constants $b, s$ and $l_{1}$ and $l_{2}$ are to be determined. Following Woodcock and Engel (2005), observations are treated here as truth, and observation error is ignored, i.e. the bias correction constant $(b)$ is simply the model bias relative to observations, and the slope $(s)$ and linear $\left(l_{1}\right.$ and $\left.l_{2}\right)$ constants are determined using least squares regression assuming no error in the observation, the latter being forced through the origin. This is of course not the case, and strictly speaking, observational error should be accounted for in the regression analysis (e.g. Tolman, 1998a). For the purposes of deriving unbiased winds to force the wave model, such effects are deemed small enough to ignore here.

Throughout this chapter, corrections are determined for, and applied to, short term wind forecasts only, i.e. the same 0-12 lead time forecast winds that were examined in Chapter 3.

\subsection{Results}

Results are presented in three stages. Section 5.4.1 presents results from simple corrections to the whole field, the application of spatially variant corrections are then explored in Section 5.4.2, and finally, results for spatially and temporally varying learned corrections are presented in Section 5.4.3. In all cases, verification statistics for the corrected winds are calculated as in Chapter 3. Global verification statistics refer to statistics calculated from the full set of scatterometer and (corrected) model co-locations. It is worth noting that in the case of the static homogeneous (Section 5.4.1) and spatially varying (Section 5.4.2) results, the data used to determine the corrections is the same data used to verify the corrected winds, and strictly speaking, cannot be considered independent. In the case of the learned correction, the use of past observation-model comparisons to correct the current forecast time has the advantage of maintaining the independence of the verifying data, with data valid at a given time step having not been used in the construction of the correction applied to that time step.

\subsubsection{Static and Homogeneous}

Corrections calculated as described in Section 5.3 using the entire data set yield the following wind adjustments: 


$$
\begin{array}{ll}
\text { Bias: } & U_{c}=U+0.52 \\
\text { Slope: } & U_{c}=1.06 U \\
\text { Linear : } & U_{c}=1.02 U+0.38
\end{array}
$$

where $U$ is the model wind speed and $U_{c}$ is the corrected wind speed. Table 5.4.1, shows global verification statistics for each of these corrections. RMSE has been reduced by between $5-6 \%$ and the bias has been removed in all cases. SI does not show much sensitivity. This is not unexpected from these simple systematic corrections for the whole period. The linear correction performs best, but results are marginal.

Table 5.1: Global verification statistics for ACCESS $U_{10}$ against QuikSCAT data before and after the application of homogeneous corrections calculated retrospectively over the entire four month period.

\begin{tabular}{lrrr}
\hline Run & Bias & RMSE & SI \\
\hline Uncorrected & -0.524 & 1.568 & 0.183 \\
\multicolumn{4}{c}{ Simple bulk corrections } \\
Bias & -0 & 1.478 & 0.183 \\
Slope & -0.068 & 1.485 & 0.184 \\
Linear & -0 & 1.477 & 0.183 \\
\hline
\end{tabular}

Examining the spatial bias, however, highlights the danger of considering global statistics alone. The case of a slope correction is used for illustration here, which amounts to a $6 \%$ increase in $U_{10}$. Figure 5.1 shows the spatial wind bias for the whole period after applying this correction. Despite the average bias being close to zero, significant regional biases remain in the open ocean. Strong negative biases still remain in coastal regions.

\subsubsection{Spatially Varying Corrections}

The effective removal of the spatial bias requires a spatially varying correction. The construction and performance of such corrections is examined in this section. The value of these corrections are assessed in terms of the statistical gains they offer over the static homogeneous corrections above, as well as their ability to 


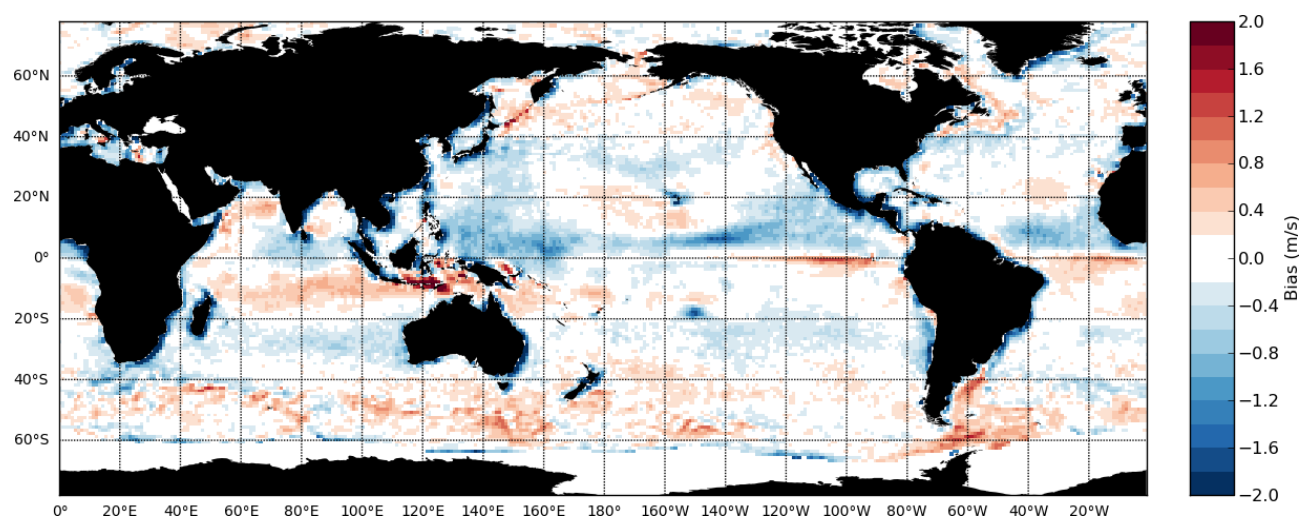

Figure 5.1: Spatial $U_{10}$ bias after increasing $U_{10}$ uniformly by $6 \%$.

produce spatially unbiased winds, in line with the overall aims of this thesis. The main purpose here is to explore some aspects of these spatial corrections before applying them in the context of a learned correction scheme.

The homogeneous corrections applied above considered only the scalar wind speed. Wind is of course a vector quality, and also contains directional error. For global homogeneous corrections, this is justified by the fact that the mean directional bias over the globe is small (e.g. Table 3.1). When considering regional corrections, directional effects may play a larger role. In the case of a coastal bias, for example, the severity of the bias may be expected to differ for off-shore, onshore or along-shore conditions.

This is explored here for a number of distinct regions, shown in Figure 5.2. Figure 5.3 shows wind roses for both the modelled wind (left) and the observations (middle), based on all co-locations within each specified area. The right panels show directionally dependent slope correction. These are constructed by binning co-locations into $20^{\circ}$ directional bins according to observation direction, and calculating the slope correction for each bin. Where a given $20^{\circ}$ bin contains less than $1 \%$ of the total observations, no slope is plotted. The blue line shows, for reference, the slope calculated from all co-locations in the region, irrespective of direction. Note that wind roses show direction the wind is travelling to, consistent with oceanographic conventions.

A correction based only on the scalar wind speed will work well where (a) the directional distribution in the wind roses for modelled and observed values do not significantly deviate from one another and (b) the slope correction does not vary significantly with direction. In the plots in Figure 5.3, where the directionally 


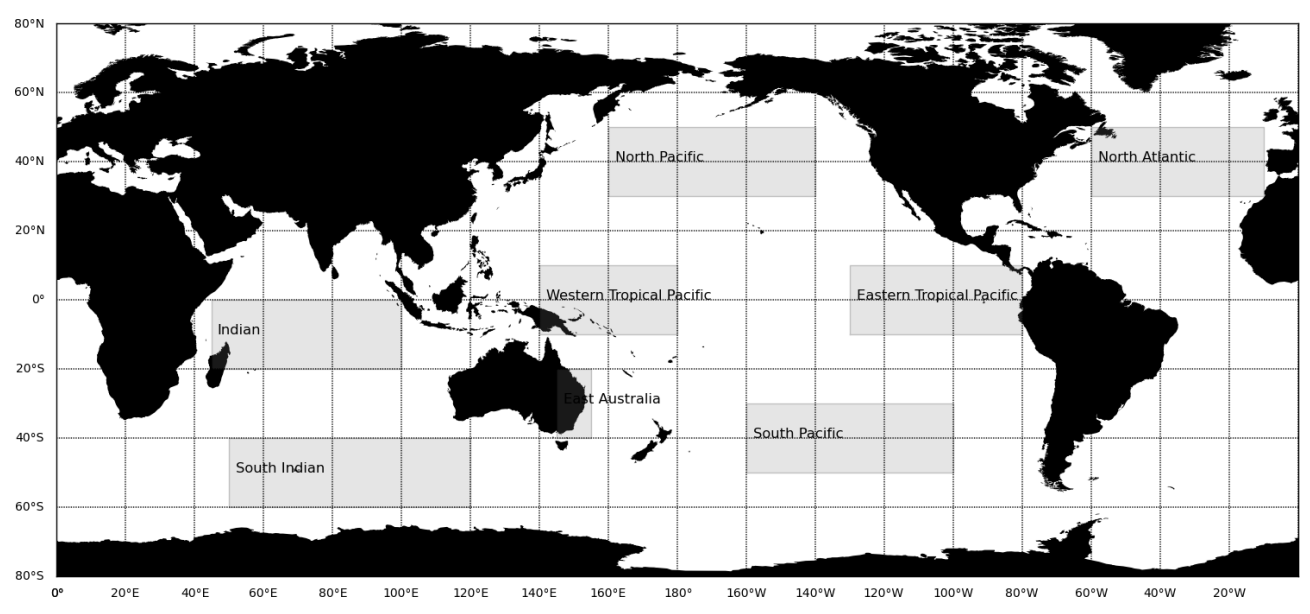

Figure 5.2: Regions for which wind roses and directional dependence of slope are calculated. See Figure 5.3.

dependent slope correction (red line) matches the overall slope correction (blue line), correcting the scalar wind speed is likely to produce a good result in all conditions.

On the whole, the directional distribution appears to be well captured by the model in all regions. The directionally dependent slope correction for the Tropical Pacific regions, Indian Ocean and the South Indian regions show little variation in the slope in the range of directions for which the wind is blowing for the majority of the time. Some variation in slope with direction is seen in the mid-latitude corrections however. For the North Pacific and North Atlantic a minimum in the slope correction occurs for winds blowing in North-Easterly directions. In the South Pacific this minimum is to the South-East. In these midlatitude regions, the maximum slope correcton is located in approximately the opposite direction to the minimum. These differences between the Northern and Southern Hemisphere mid-latitudes, combined with the directional bias shown in Figure 3.10, suggest some interplay here with the large meridional circulations of the atmosphere. Further exploration of this is out of scope here.

One means of accounting for a directionally dependent bias is by applying different corrections to the $u$ and $v$ components, as done by Greenslade et al. (2005). Such separate $u$ and $v$ corrections can account for symmetric east-west or north-south variations. In the directionally dependent slope plots of Figure 5.3, errors of this nature would present as an ellipse rather than a circle. Such corrections cannot, however, account for a maximum in one direction and a minimum in the opposite direction, as in the off-centre circles seen in these mid-latitude 


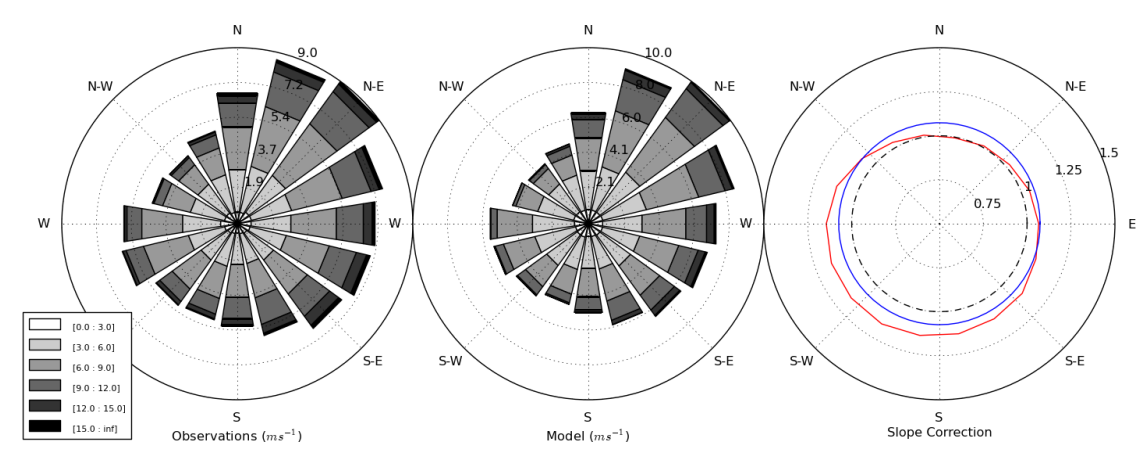

(a) North Pacific

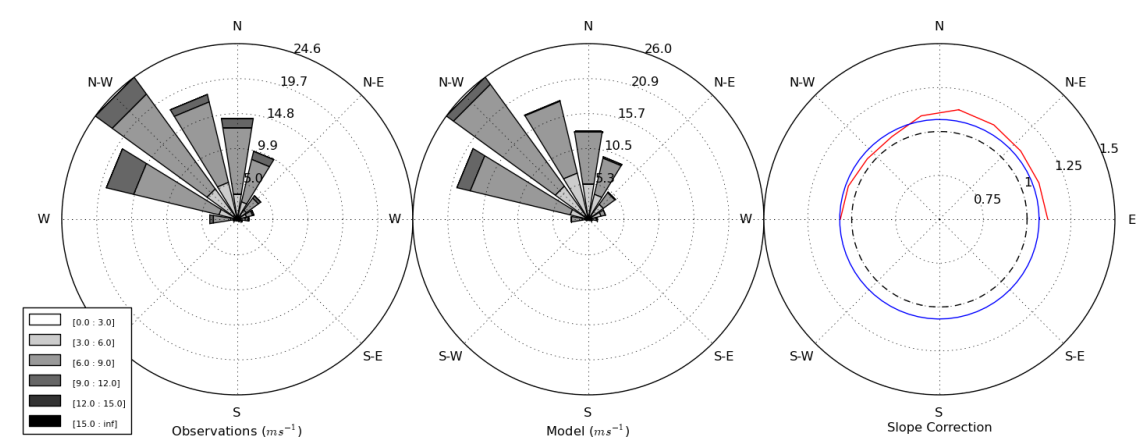

(b) Eastern Tropical Pacific

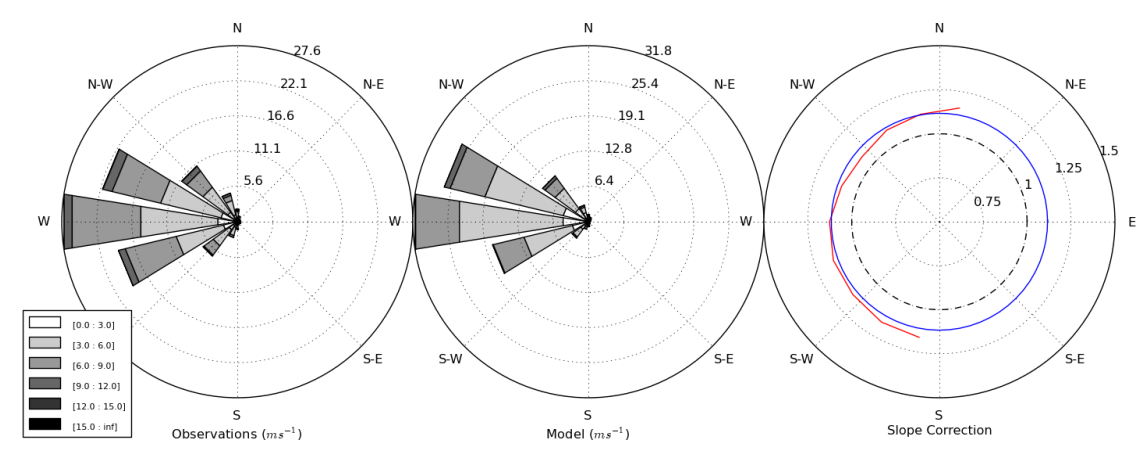

(c) Western Tropical Pacific

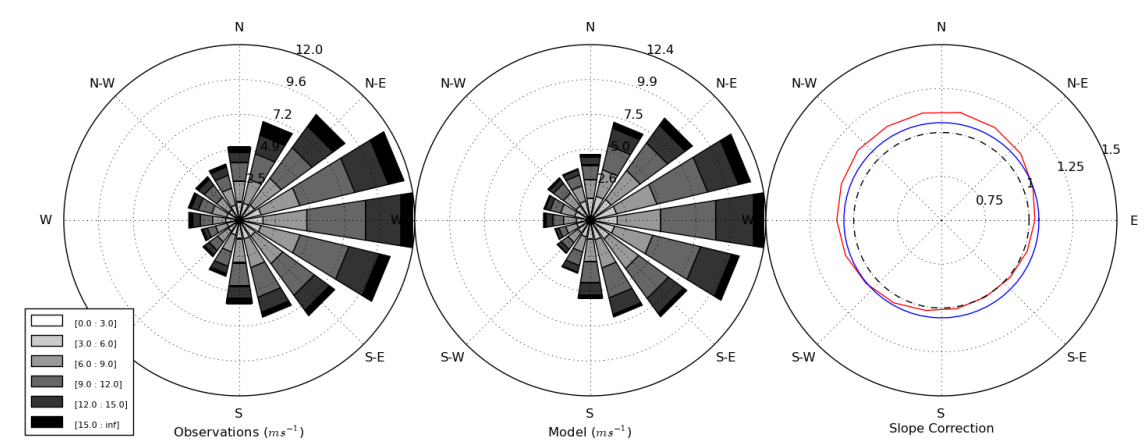

(d) South Pacific

Figure 5.3: For each region shown in figure 5.2, the left panels show model wind roses, the middle panels show observation wind roses and the right panels show the directionally dependent slope correction, constructed by binning co-locations into $20^{\circ}$ directional bins according to observation direction, and calculating the slope correction for each bin. 


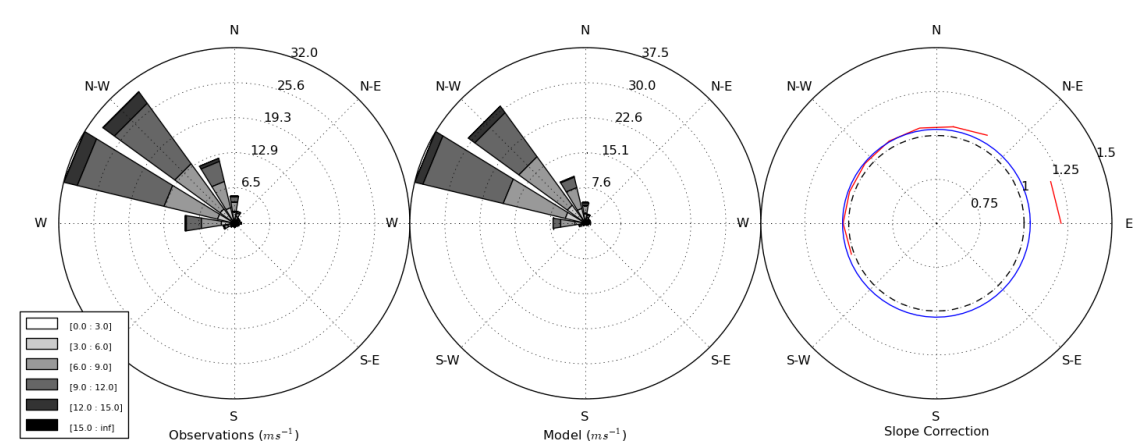

(e) Indian

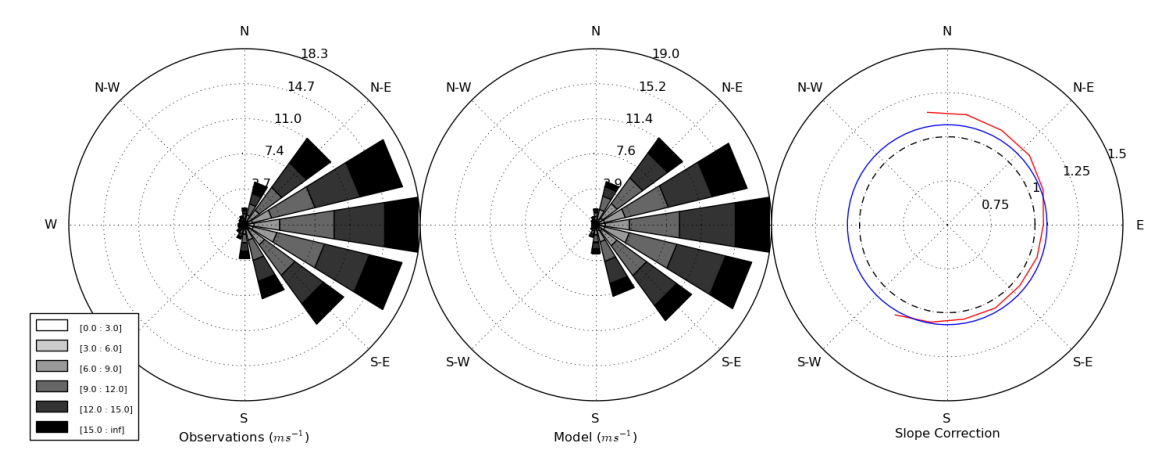

(f) South Indian
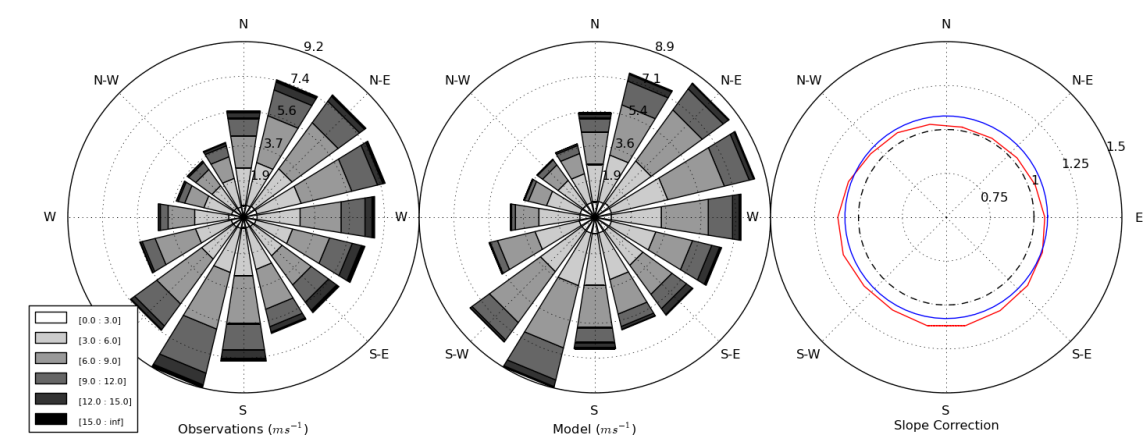

(g) North Atlantic

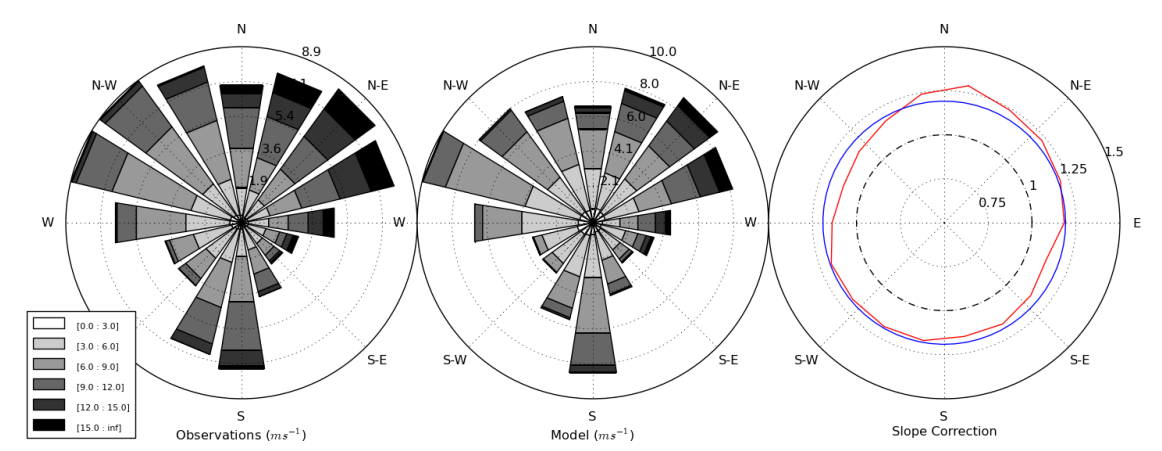

(h) East Australia

Figure 5.3: Continued. 
cases. As such, considering $u$ and $v$ components separately is unlikely to provide improvements over simpler, scalar wind speed corrections. In any event, these mid-latitude directional slope variations are minor.

As previously discussed, the directionally dependent bias might be expected to be most prevalent at the coast, with off-shore and on-shore wind error characteristics varying. In the case of the Australian east coast (Figure $5.3(\mathrm{~h})$ ), indeed some variation is apparent, though again, it is relatively minor.

Proceeding then with scalar wind speed corrections, co-location data for the whole period are binned into $1 \times 1^{\circ}$ latitude-longitude bins, and appropriate corrections determined for each bin, producing an independent correction to be applied at each model grid point. The resulting correction coefficients (i.e. those corresponding to equations 5.5 - 5.7) for bias (a), slope (b), and linear (c and d) corrections are shown in Figure 5.4. As expected from the spatial variation of the wind speed bias (e.g. Figure 3.7), these are inhomogeneous in space.

Global verification statistics for $U_{10}$ after the application of these spatially varying corrections are presented in Table 5.2. Overall, significant improvement is seen in comparison with the static homogeneous corrections. Slope corrections show the best overall results, though again the difference between these methods is small. The simplicity of having to only calculate a single correction field favours the use of slope corrections over linear corrections.

Table 5.2: Global verification statistics for ACCESS $U_{10}$ against QuikSCAT data before and after the application of spatially varying corrections calculated retrospectively over the entire four month period.

\begin{tabular}{lrrr}
\hline Run & Bias & RMS & SI \\
\hline Uncorrected & -0.524 & 1.568 & 0.183 \\
\multicolumn{4}{c}{ Spatially varying } \\
Bias & -0 & 1.428 & 0.177 \\
Slope & -0.037 & 1.426 & 0.176 \\
Linear & -0.044 & 1.435 & 0.178 \\
\hline
\end{tabular}

Perhaps more importantly for the purposes of this work, applying spatially varying corrections effectively removes the bias over the whole global domain. Figure 5.5 shows the $U_{10}$ bias after applying the slope corrections of Figure 5.4 (b). The broad-scale regionally variant biases have been almost eliminated, and 

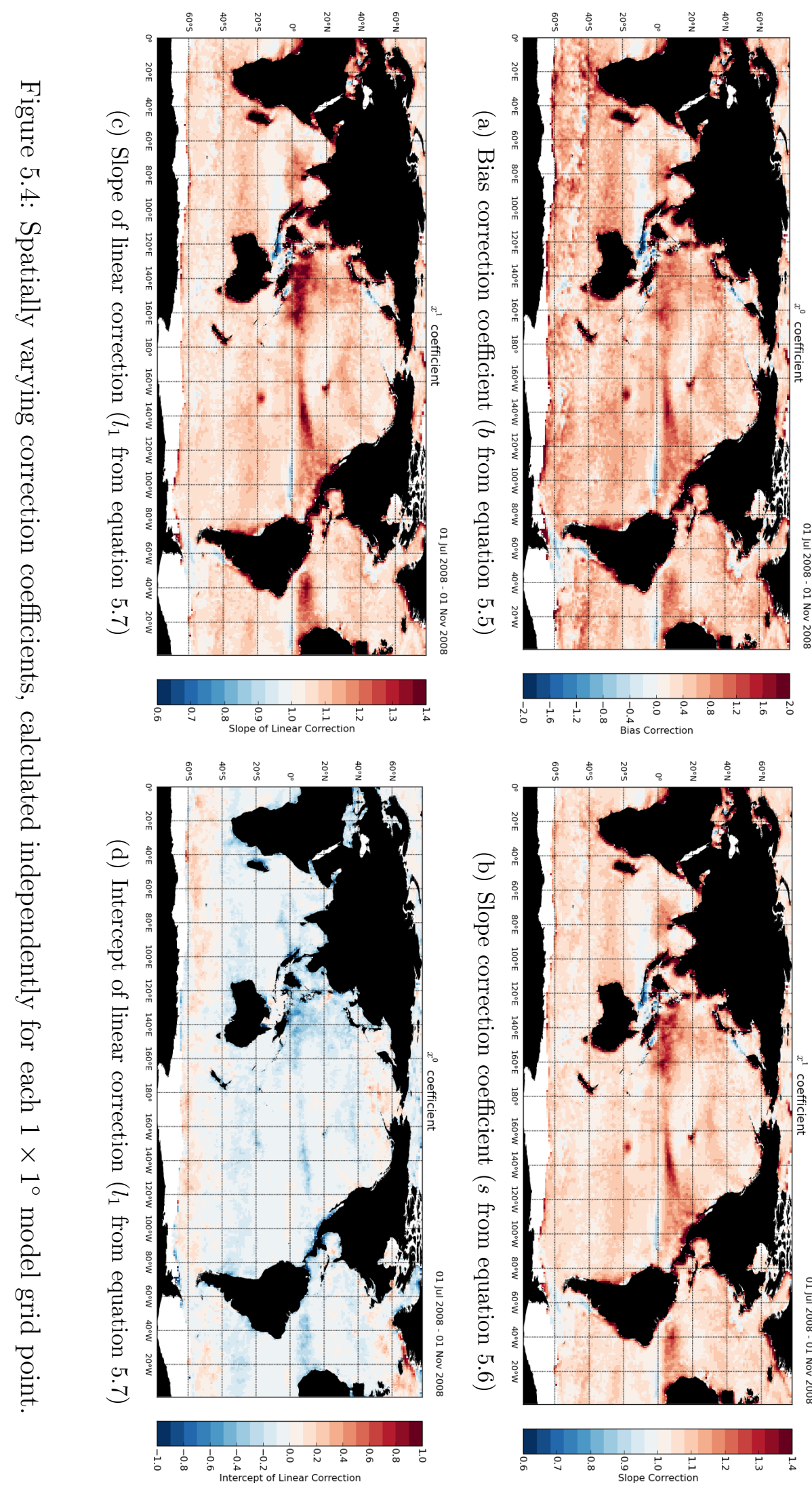
the finer scale coastal biases have been greatly reduced. At any given grid point, the removal of the $U_{10}$ biases is not surprising, given that verifications are based here on the same scatterometer data used to determine the correction. However, for the purposes of reducing bias over the entire domain, it is clear that spatially varying corrections offer significant gains over homogeneous corrections.

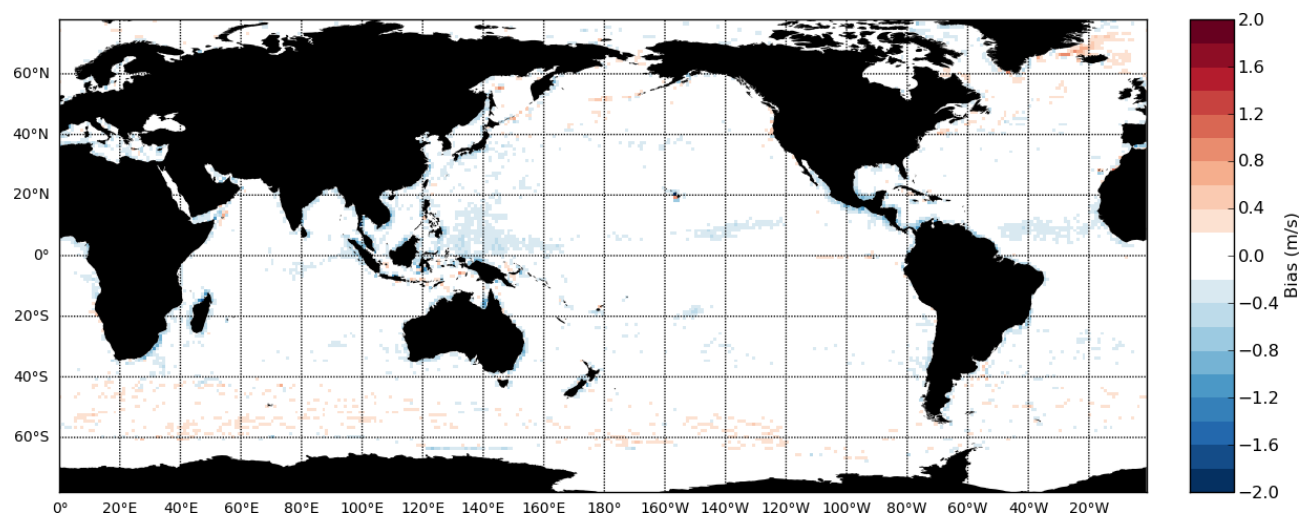

Figure 5.5: $U_{10}$ bias after applying the spatially variant slope correction shown in Figure 5.4 (b)

In summary, the corrections based on scalar wind speed only appear reasonable, with more complex, directionally dependent corrections unlikely to offer significant gains. Accounting for the regional variation in model bias is effectively addressed by applying spatially varying corrections. Corrections that are a function of the $U_{10}$, i.e. slope and linear corrections, were found to perform better than bias corrections, with the small difference between the former two favouring the simpler, slope correction. The need to manually monitor these corrections remains. This is addressed in the following section, examining the application of learned corrections.

\subsubsection{Spatially and Temporally Varying Learned Correc- tions}

As described in Section 5.3, the method used here relies on the calculation of corrections based on a moving window of historical data. Spatially varying corrections are calculated as above at each model time, with the period used to calculate the correction grid restricted to a given temporal window prior to this time. For example, if a 30 day learning window is used, the correction to be applied to the $00 \mathrm{Z}$ forecast for July $1^{\text {st }}$ would be calculated from co-locations 
for the period April 31 ${ }^{\text {st }}, 21 \mathrm{Z}$ to June 30, 21Z. The correction of $03 \mathrm{Z}$ would use data from June $1^{\text {st }}, 00 \mathrm{Z}$ to July $1^{\text {st }} 00 \mathrm{Z}$, etc. This is schematically represented in Figure 5.6. Updating the correction at every time-step in this way produces a correction that automatically evolves to match the recent historical bias of the model.

Based on the results of the previous section, spatially varying slope corrections of the scalar wind speed are used. The time step from one correction calculation to the next is three hours, the period at which the model output is available. The training window for each time step ends at the previous time step, i.e. three hours prior (the time between $t$ and $t$-1 in Figure 5.6 (a) for example).

When considering correction methodologies such as the learned corrections proposed here, some thought must be applied to the scales being targeted. As described above in Section 5.3 regarding the OCF methodology, the error in a model forecast can be somewhat simply described as having both a systematic bias component, and a random error component. These errors though, occur at a range of temporal and spatial scales.

In the context of synoptic scale weather systems, a distinction can be drawn between amplitude and phase error. The former is the model's ability to capture the intensity of a system in a Lagrangian sense, or the variance of a signal in a Eulerian sense. The latter describes the skill of the placement of synoptic features, experienced as the timing in a fixed location. In the imagined case of a low pressure system that is predicted with the correct intensity, but the timing is delayed by several hours, a location may experience a negative bias as it approaches, and a positive bias after it passes over. In the context of the corrections applied here, this would be attributed as random error. This technique is not able to remove this type of error, rather, systematic bias on the scale of weeks to months is the target.

The most obvious means of targeting these larger scale systematic errors is to increase the length of the training window. Figure 5.7, for example, shows slope corrections for August $1^{\text {st }}$, 2008, calculated from learning windows of 2, 5, 10 and 30 days. The spatial coherency of the corrections increases with the length of the learning window as the influence of phase error decreases. The total number of observations contributing to the correction calculation at each grid point (Figure 5.8) also increases with window size, with implications for statistical robustness.

An alternate method of targeting synoptic scale systematic bias, is to increase 


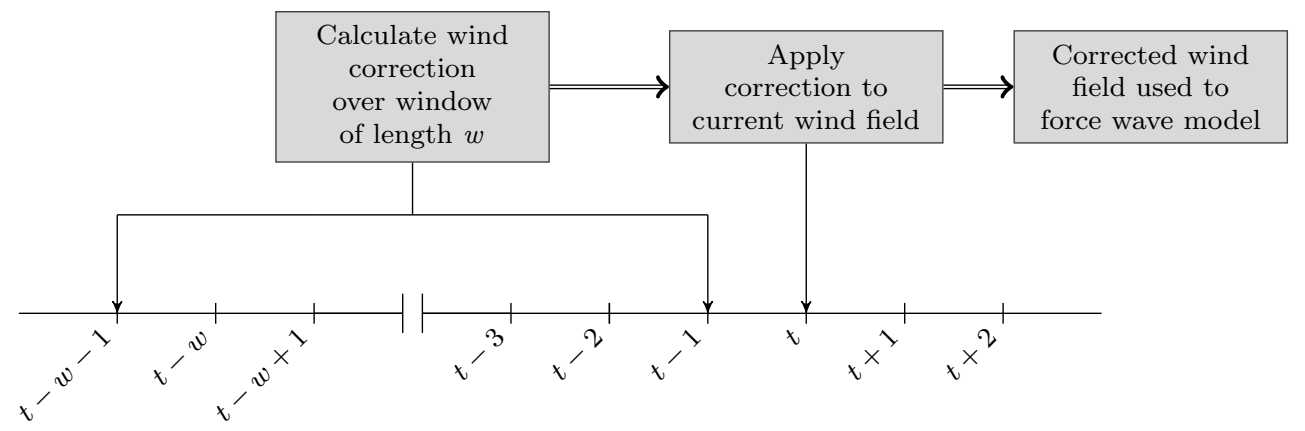

(a) $t=0$

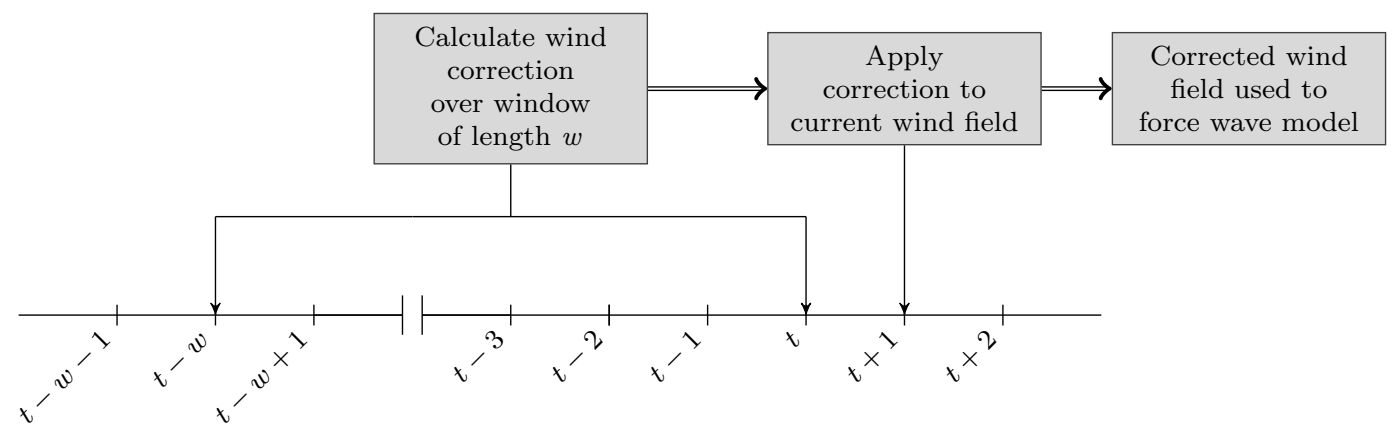

(b) $t=1$

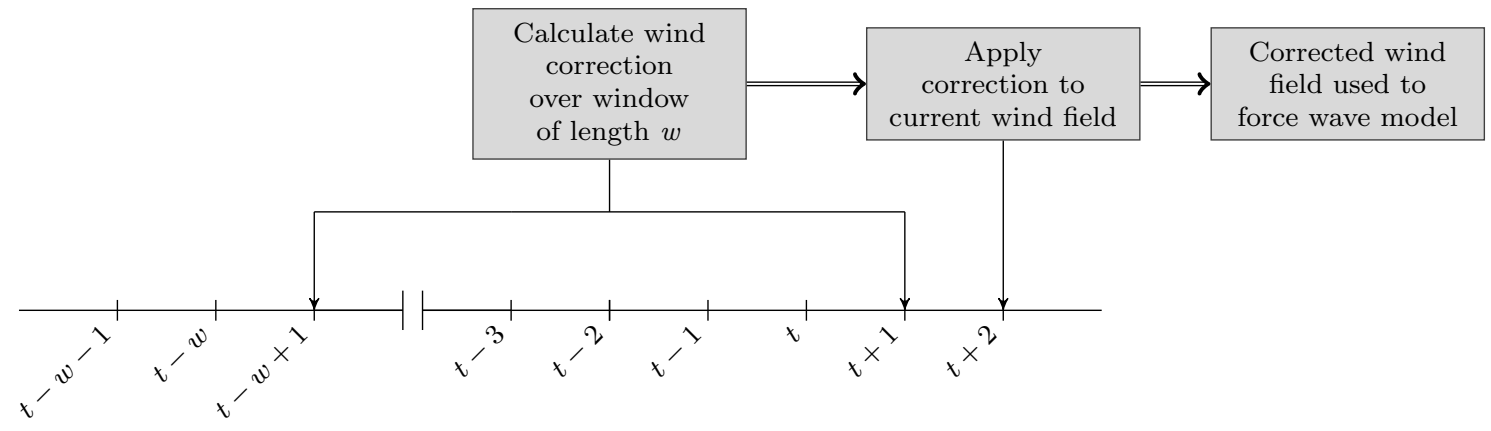

(c) $t=2$

Figure 5.6: Schematic of the learned correction method. $w$ represents an arbitrary learning window. Time steps of 3 hours used in this work, with with the calculated correction varying geographically well as time, see text for details. 


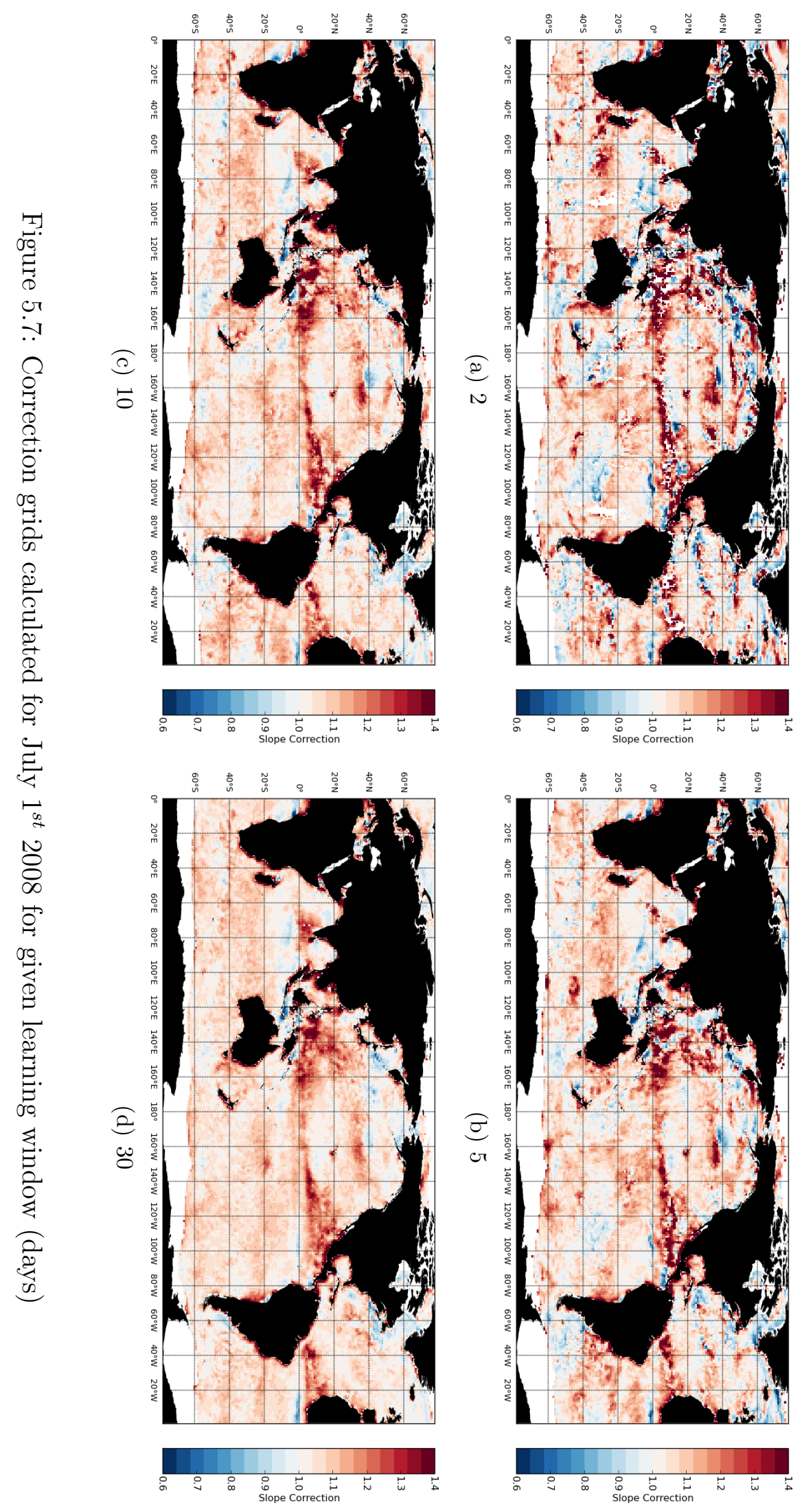



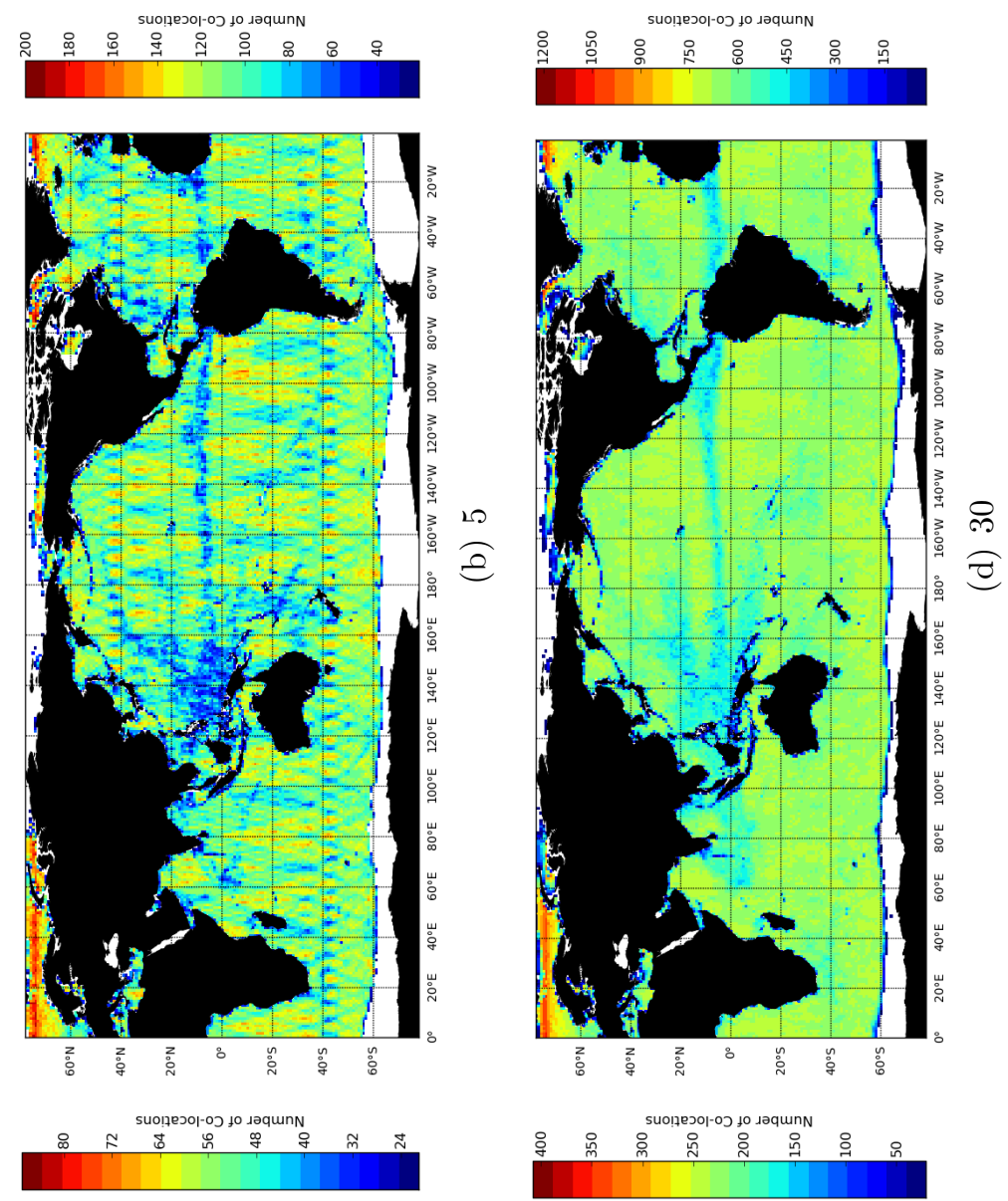

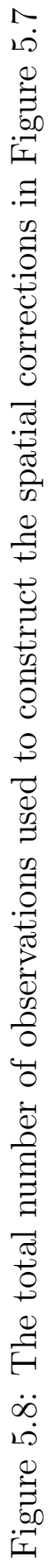
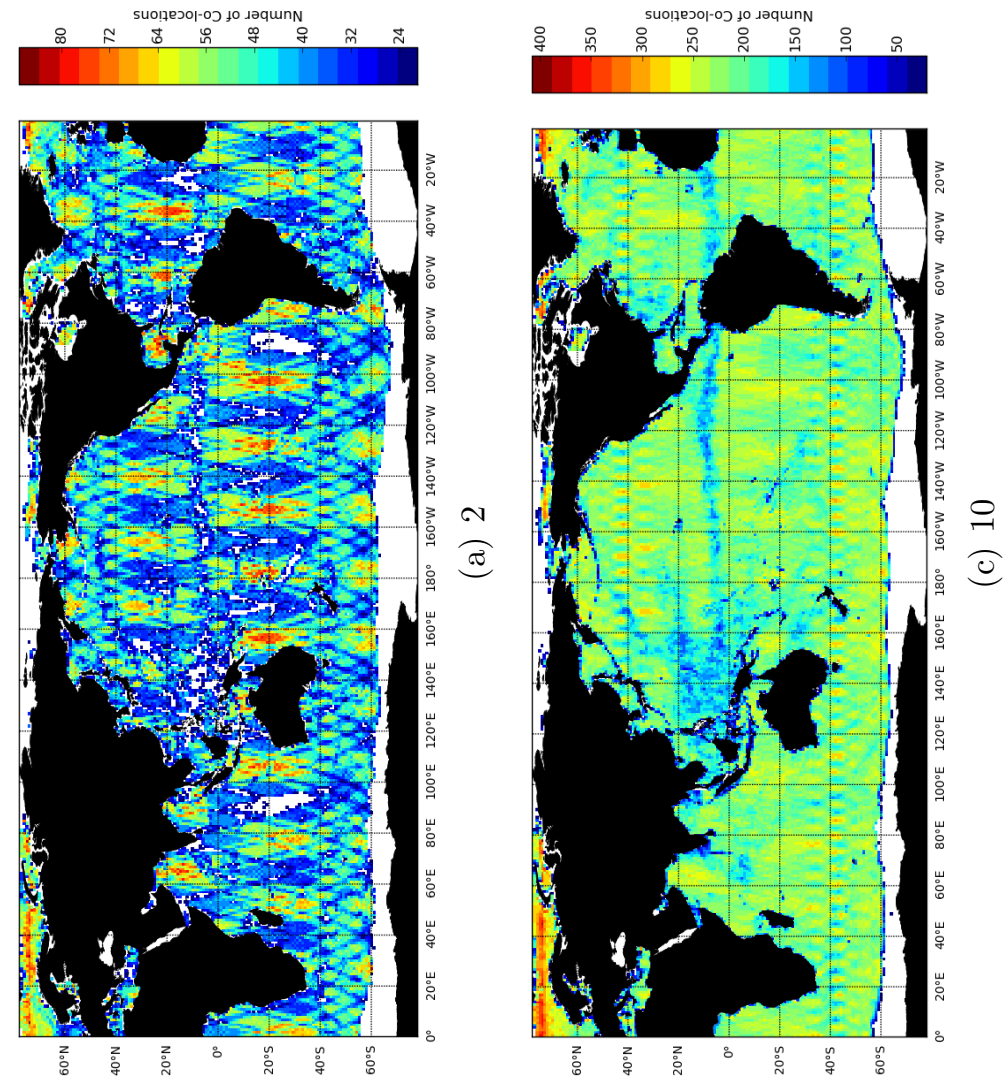
the area over which a correction is calculated. Returning to the hypothetical case of a mis-timed low pressure system, calculating the error over the area of the whole system targets the larger scale systematic biases in the same way as increasing the temporal window. There are a number of ways this could be achieved. The most obvious is to use larger boxes when determining the corrections. For example, rather than the $1 \times 1^{\circ}$ boxes used here, $10 \times 10^{\circ}$ boxes could be used, applying the calculated correction to every grid point in that box. However, this could potentially lead to significant discontinuities at the box edges, with the applied corrections being visibly 'patchy' over the globe. This could be overcome by calculating independent corrections for each grid point, based on the data from the $10 \times 10^{\circ}$ box surrounding that grid point. Alternatively, some spatial smoothing can simply be applied to the $1 \times 1$ degree corrections already calculated. This achieves the same thing, while requiring no further correction calculations, allowing a number of different 'spatial windows' to be explored relatively easily. Gaussian spatial filtering is used here, giving the added advantage of applying decreasing weight to surrounding grid points with increasing distance.

Figure 5.9, shows a correction grid calculated with a 10 day learning with smoothing applied over $2^{\circ}, 5^{\circ}$ and $10^{\circ}$ windows. Increasing the spatial smoothing achieves qualitatively the same thing as increasing the temporal length of the window. The downside of this approach is that it reduces the ability to resolve small spatial scale features, such as those around coastlines and in the lee of islands.

Central to these considerations is the fact that learned corrections are applied to a subsequent forecast. Corrections calculated over small temporal and spatial scales can be expected to vary on similar scales, making them inappropriate for this type of application. Conversely, the strength of a learned correction scheme is its ability to automatically adapt to the evolution of systematic error on monthly and seasonal timescales, or indeed with changes due to alterations to the atmospheric model. Increasing the length of the learning window decreases the speed with which the correction can adapt to these changes. Thus, a balance must be struck when choosing the scales of the learning windows.

Learning windows of 2, 5, 10, 20 and 30 days with both no smoothing (i.e. calculating corrections for each model grid point), and smoothing of 2, 3, 5, 10 and 20 degrees are explored here, resulting in 30 different combinations of learning window characteristics. Corrected winds for the whole four month period were 

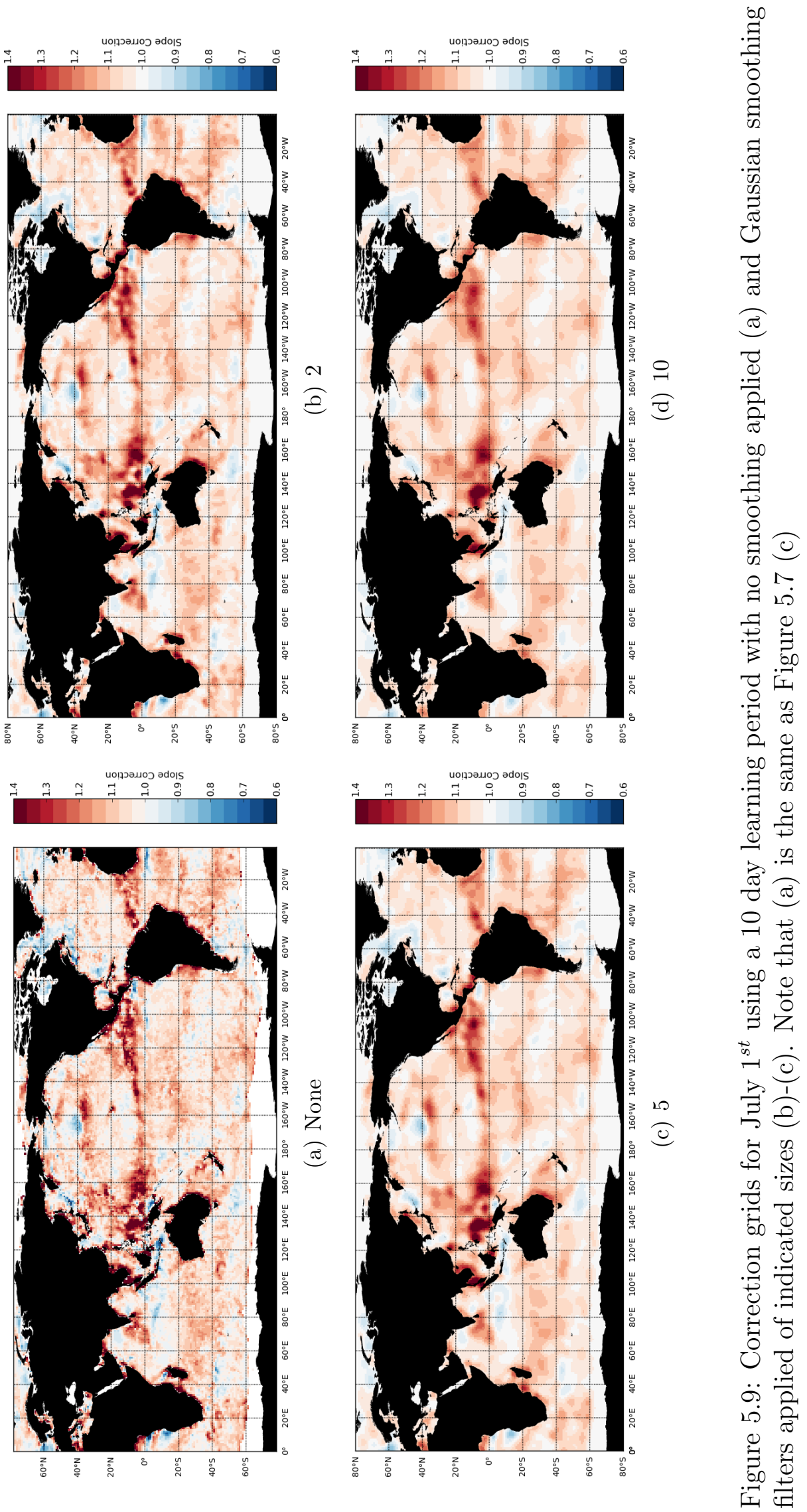
calculated and verified for each case. Figure 5.10 shows the percentage reduction in $U_{10}$ RMSE associated with each combination.

Consistent with the considerations discussed above, applying corrections corresponding to small time and space scales, results in very little improvement, with SI in fact being degraded (not shown). For short time windows, increasing the spatial scales results in significant improvement, however, this trend is not apparent for learning windows of 5 and 10 days, and reverses for longer learning windows. This is consistent with the increased smoothing reducing the ability to resolve small scale features such as those along coastlines and in the lee of islands, as seen in figure 5.9. Overall, the best results are achieved by using a long, 30 day learning window to target the desired error scales, with no spatial smoothing applied, maintaining the ability to correct systematic errors on small spatial scales.

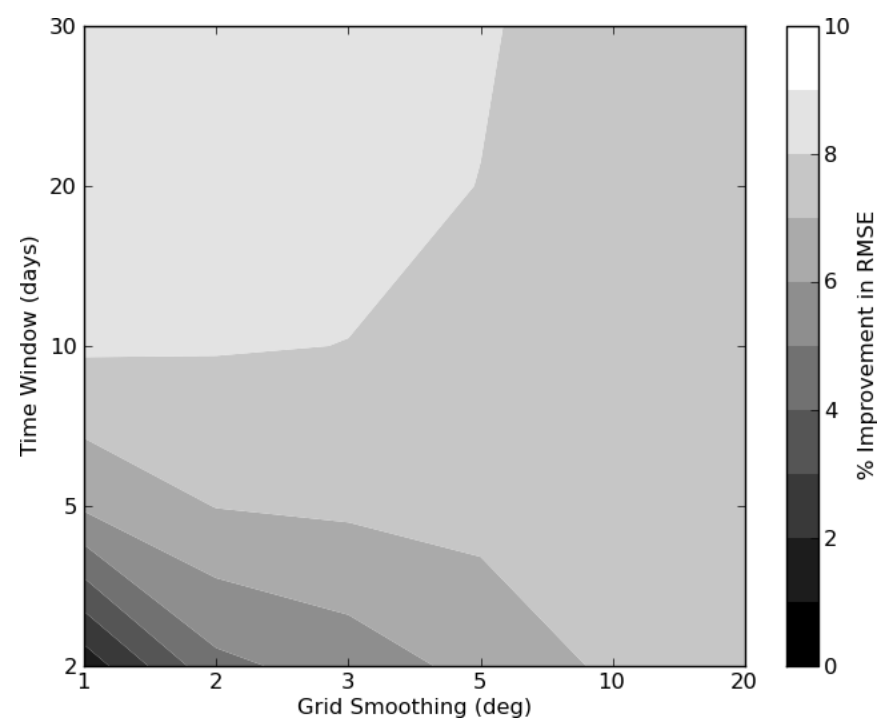

Figure 5.10: Percentage improvement in RMSE relative to scatterometer data for corrected wind fields over the uncorrected wind field for different length learning windows and different levels of smoothing applied to the calculated correction field.

The correction applied to each forecast grid here is calculated over the window ending three hours prior, with a separate correction grid calculated for each 3 hourly time-step. In an operational environment, runs are performed typically every 6 to 12 hours. The gap between the end of the training window, and the forecast being corrected would thus increase accordingly. If it is assumed, for example, that the atmospheric model is run every 12 hours and that scatterometer 
data is available with minimal delay behind real time. Then, in the case of the short term forecasts used here, the correction calculated for the period ending at $00 \mathrm{Z}$ would be applied to all forecasts from 3 to 12 hours, thus increasing the average time between the end of a learning window, and the model time to which the correction is applied.

To assess the impact of this change, the analysis above was repeated, calculating corrections only every 12 hours, and applying this correction to the subsequent four 3 hourly timesteps. Figure 5.11 shows the percentage improvement in $U_{10}$ RMSE, comparable to Figure 5.10 where corrections are calculated every 3 hours. Consistent with the persistence of errors associated with longer time periods, this change results in greater degradation at shorter time/space scales than longer ones. The superiority of longer windows is even stronger here, with little reduction in the effectiveness using windows at these scales.

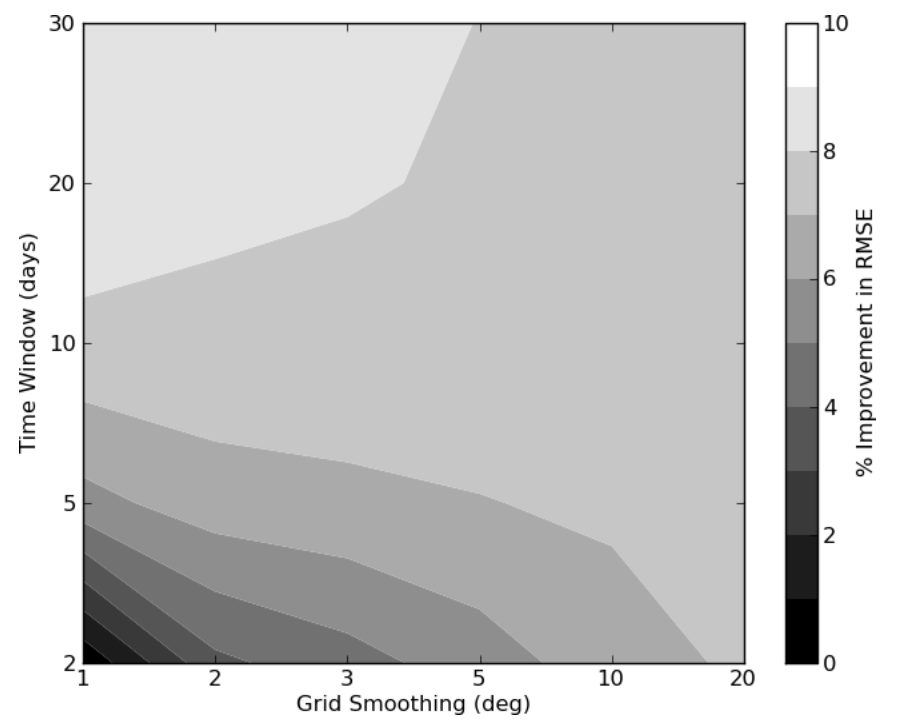

Figure 5.11: As in Figure 5.10, but with operational timings. See text for details.

For the remainder of this work, corrections are calculated and applied every 3 hours. A 30 day learning window is used to target the desired error scales, with no spatial smoothing applied, maintaining the ability to correct systematic errors on small spatial scales. The evolution of these corrections is shown in Figure 5.12, at monthly intervals, over the period examined in this work. This evolution is slow and relatively slight when considering learning windows of 30 days. This is not unexpected, given the small variation in spatial bias from month to month over the period of this study (e.g. Figure 3.9). 


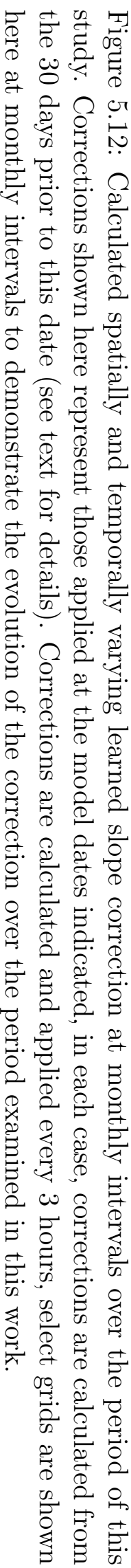
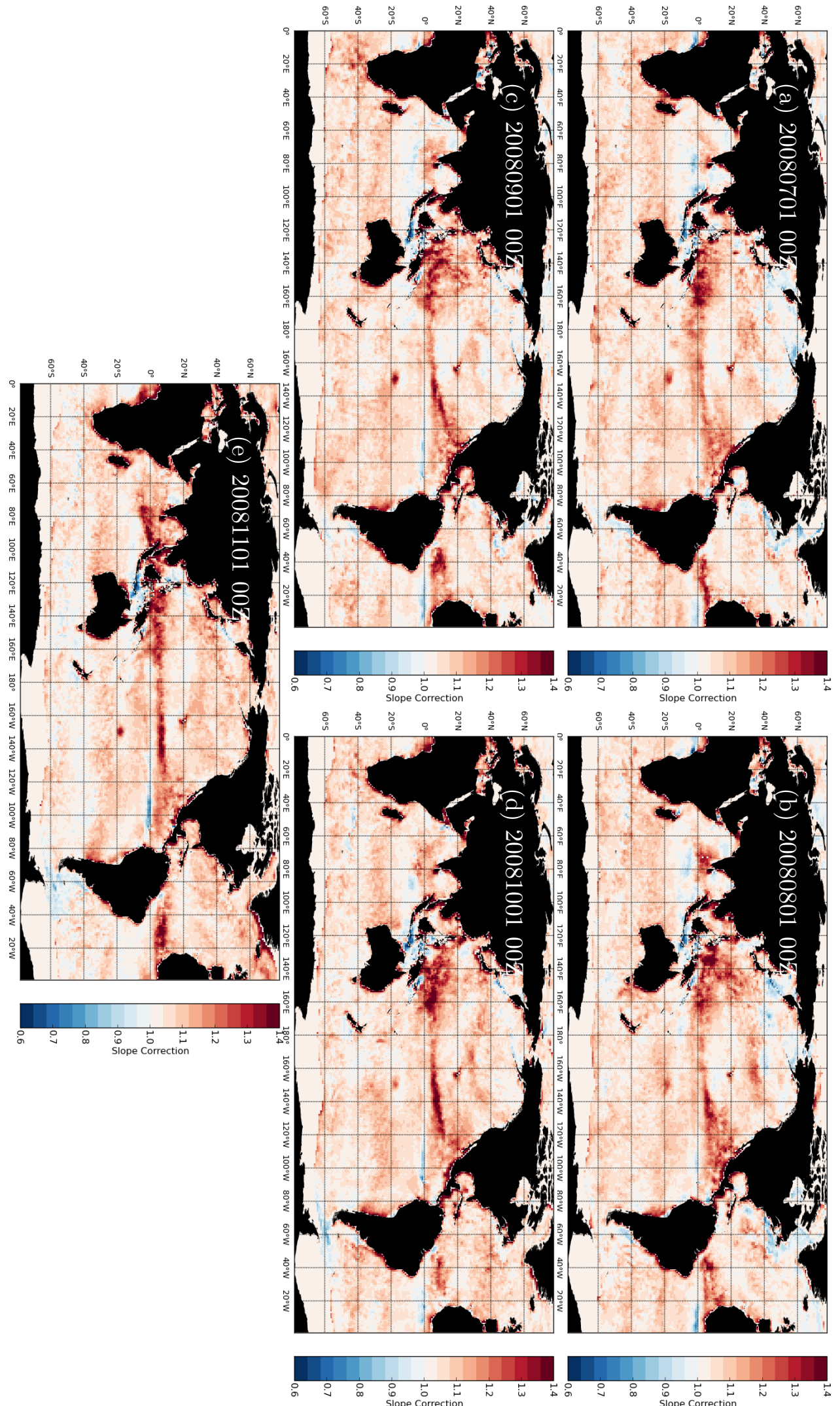
Table 5.3: Global verification statistics for ACCESS $U_{10}$ against QuikSCAT data before and after the application of spatially and temporally varying learned corrections.

\begin{tabular}{lrrr} 
& Bias $(\mathrm{m})$ & RMSE $(\mathrm{m})$ & SI \\
\hline Uncorrected & -0.524 & 1.568 & 0.183 \\
Corrected & -0.049 & 1.437 & 0.178 \\
\hline
\end{tabular}

Global $U_{10}$ verification statistics following the application of these corrections are given in Table 5.3. Overall, results are comparable to those of the spatially varying corrections calculated retrospectively over the entire period (Section 5.4.2). Spatial $U_{10}$ bias, shown in Figure 5.13, is similarly comparable, showing negligible remaining regional biases. This is an encouraging result, noting that verification and training data are independent here whilst the temporally fixed corrections are verified with the same data that is used to determine the corrections. Furthermore, the temporal variation of the corrections applied over the four months examined here is relatively slight. Over longer periods, where $U_{10}$ bias could be expected to show greater variation, the gains from temporally evolving corrections would be expected to be greater still.

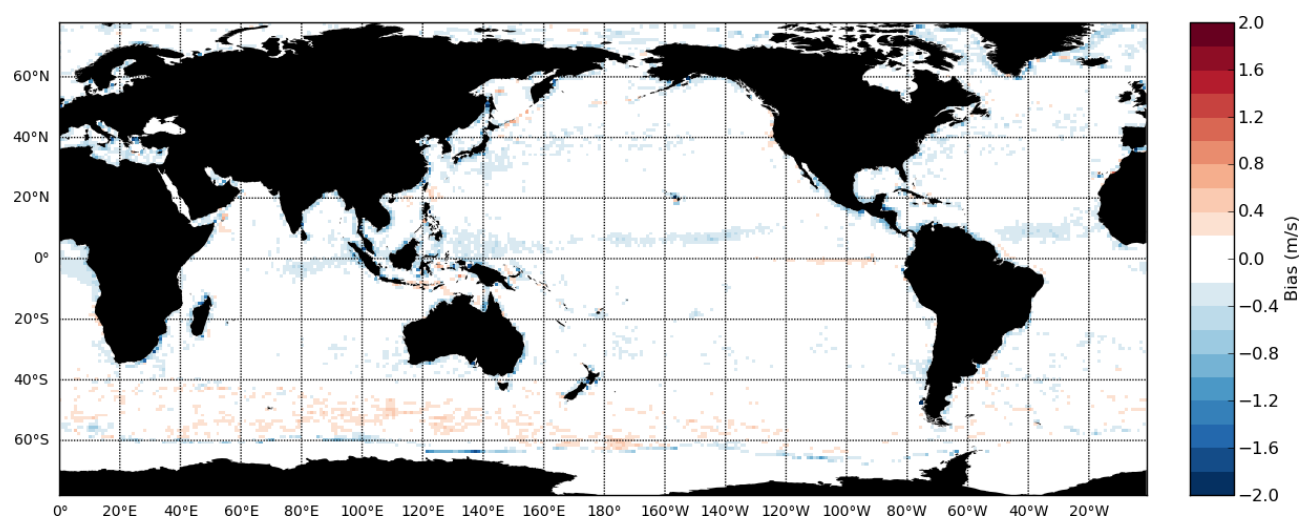

Figure 5.13: Bias of winds corrected with a 30 day learning window applied every 3 hours.

Most importantly, this method addresses the need for a spatially varying correction, whilst simultaneously removing the need to manually monitor and update that correction. Before this could be applied in an operational system, more work is needed. Issues such as real time quality control and the ability to handle data delays and drop outs are essential for practical applications. However, the clear potential exists for this approach to be applied in an operational environment in real time. It is also worth noting that QuikSCAT is no longer 
operational, however the Advanced Scatterometer (ASCAT; EUMETSAT, 2010) instrument, succeeding ERS-2, would provide a suitable replacement for an operational system.

\subsection{Discussion}

Spatially and temporally varying learned corrections based on scatterometer data have been shown above to provide a robust and effective means of correcting marine surface wind forecast data in an operational setting. A 30 day learning window was found to be the most effective at targeting systematic model biases, with independent corrections calculated for each grid point affording the removal of relatively fine scale spatial features. In the following chapter, a number of wave model runs using these corrected winds are analysed. The following section thus presents some further discussion of how the error characteristics of the winds have changed with the application of these corrections.

An overall improvement of $8 \%$ in RMSE has been achieved. Examining the spatial distribution of these gains, Figure 5.14 shows the percentage improvement in RMSE at every grid point, indicating a large amount of regional variation. In general, large improvement is seen in areas where significant biases were present in the uncorrected winds.

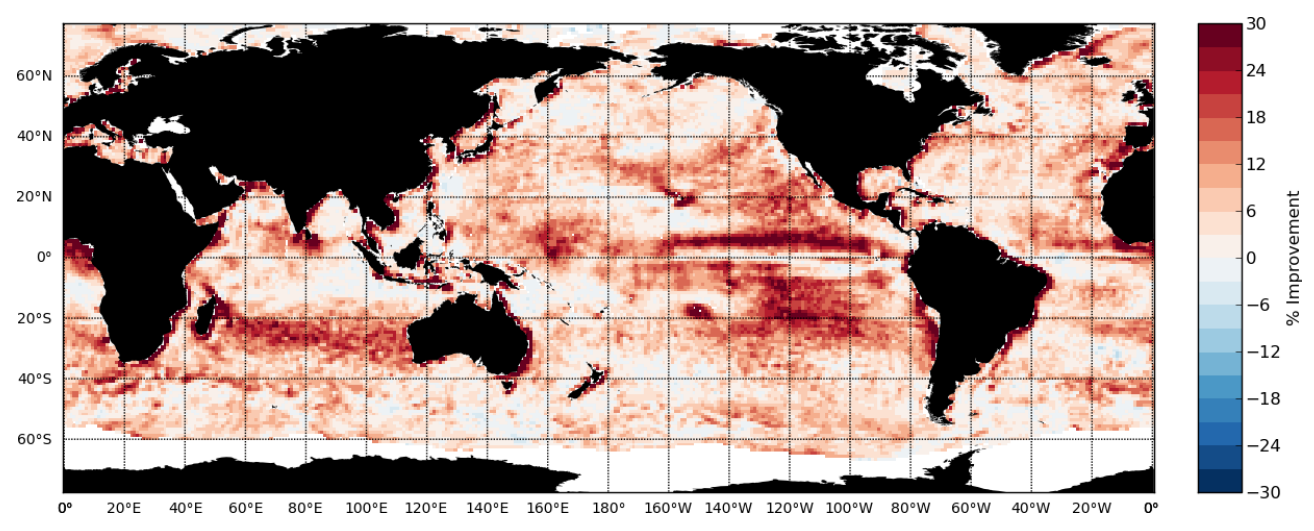

Figure 5.14: Percentage improvement in RMSE achieved by applying a learned slope correction with a window length of 30 days, and no spatial smoothing.

Table 5.4 presents statistics separately for the tropics and the extratropics of both hemispheres (North and South of $25^{\circ}$ ). Results are reasonably consistent, with slightly higher gains seen in the tropics than the extratropics. Bias is almost 
eliminated in each region.

Table 5.4: Regional statistics for $U_{10}$ relative to scatterometer data. \% improvement refers to \% improvement in RMSE.

\begin{tabular}{|c|c|c|c|c|c|}
\hline & $\operatorname{Bias}(\mathrm{m})$ & RMSE (m) & SI & $\%$ Imp. & $\mathrm{N}$ \\
\hline \multicolumn{6}{|l|}{ Global } \\
\hline Uncorrected & -0.524 & 1.568 & 0.183 & & 86181451 \\
\hline Corrected & -0.049 & 1.437 & 0.178 & 8.4 & 86181451 \\
\hline \multicolumn{6}{|c|}{ Northern Hemisphere Extratropics } \\
\hline Uncorrected & -0.493 & 1.522 & 0.209 & & 15434560 \\
\hline Corrected & -0.09 & 1.403 & 0.203 & 7.8 & 15434560 \\
\hline \multicolumn{6}{|l|}{ Tropics } \\
\hline Uncorrected & -0.523 & 1.318 & 0.175 & & 33027371 \\
\hline Corrected & -0.059 & 1.165 & 0.168 & 11.6 & 33027371 \\
\hline \multicolumn{6}{|c|}{ Southern Hemisphere Extratropics } \\
\hline Uncorrected & -0.536 & 1.536 & 0.172 & & 63624045 \\
\hline Corrected & -0.033 & 1.402 & 0.168 & 8.7 & 63624045 \\
\hline
\end{tabular}

In the context of an examination of the residual wave bias resulting from forcing with unbiased winds, reducing the overall bias to zero is insufficient to confidently attribute the bulk of the remaining $H_{s}$ error to the wave model. Error in the distribution of $U_{10}$ may remain in winds that contain no overall bias. Given the non-linear response of waves to this forcing, such wind distribution errors may still be responsible for systematic $H_{s}$ biases (e.g. Chawla et al., 2009, 2011).

Wind speed Probability Density Functions (PDFs) constructed from model/scatterometer co-locations are shown in Figure 5.15. Both observations and model PDFs are shown on the left hand side, on the right are model error PDFs (model - observed). Clear improvements in the $U_{10}$ distribution is evident in all regions from these corrections. Corrected error PDFs are closer to being centred on zero than the uncorrected cases, and the standard deviations are reduced in all regions (the error PDFs have been narrowed). A slight overestimation of $U_{10}$ in the range of about $12-20 \mathrm{~ms}^{-1}$ in the Southern Hemisphere extratropics raises some concern, especially in the context of the strong response from the waves in these wind ranges. The bulk of the over-representation in this range is likely due to the underestimation of modelled $U_{10}$ above $20 \mathrm{~ms}^{-1}$.

The underestimation of high wind speeds is more clearly illustrated in the box and whisker plots of the differences between model and observations, shown in Figure 5.16. In all regions, very low wind speeds are slightly overestimated by 

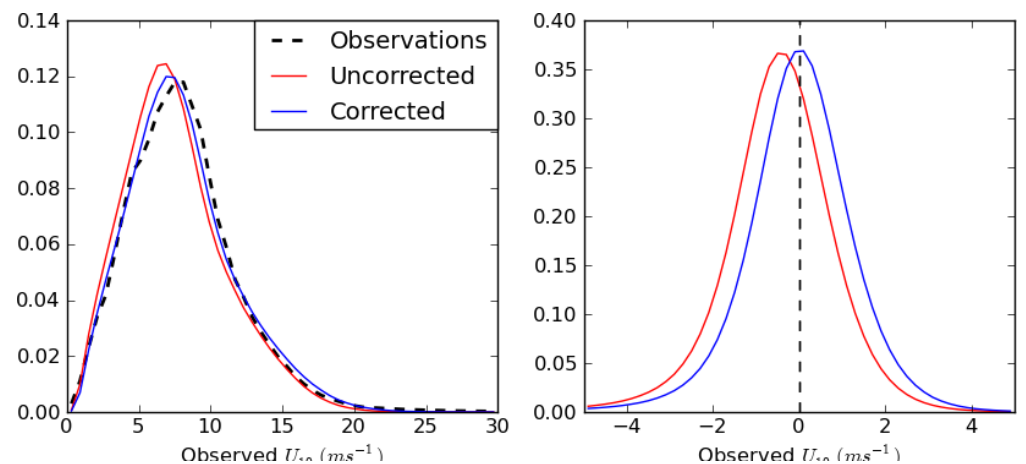

(a) Global
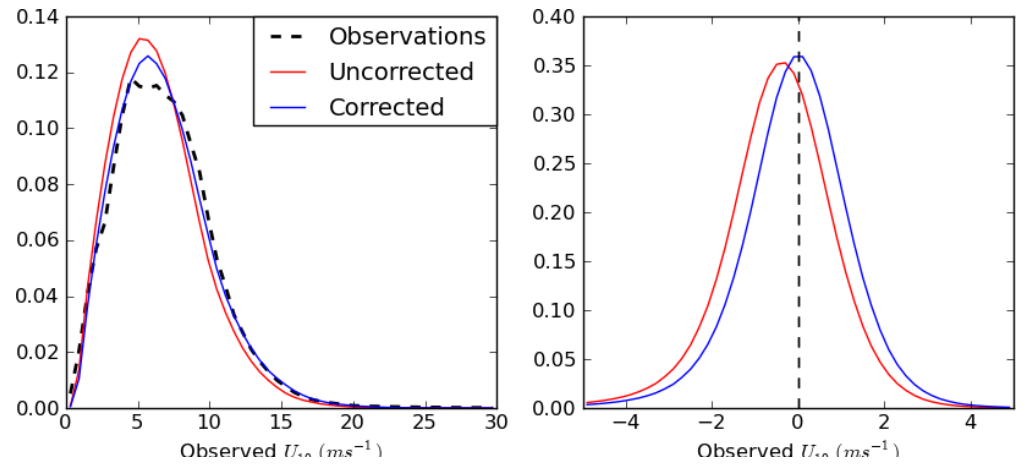

(b) Northern Hemisphere extratropics
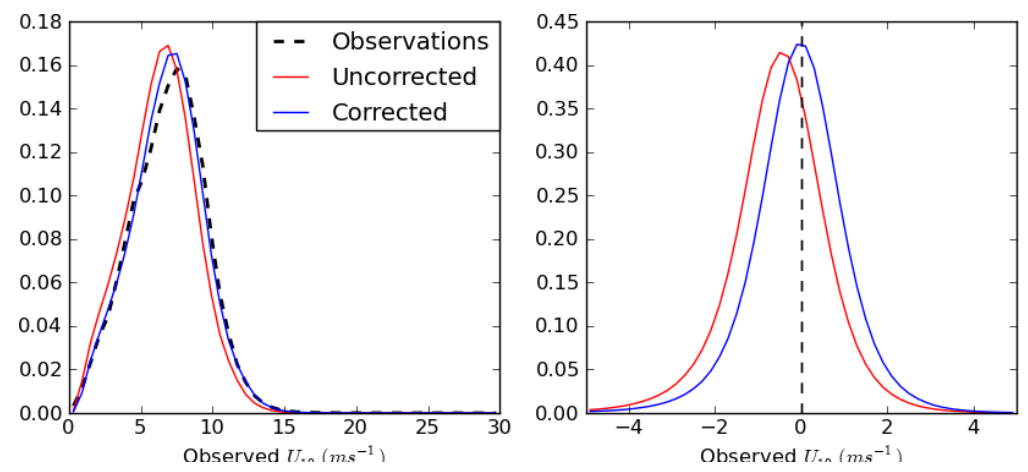

(c) Tropics
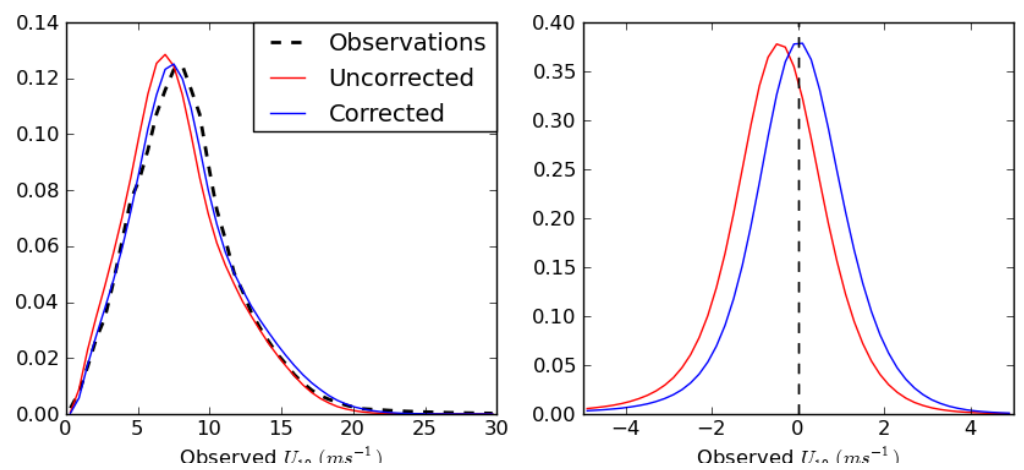

(d) Southern Hemisphere extratropics

Figure 5.15: PDFs of scatterometer observations and co-located modelled winds (left) and model errors (right) 
the model, with the corrections making this tendency worse. This is not of great concern in the context of wave generation. For wind speeds up to $18 \mathrm{~ms}^{-1}$, the corrected winds appear to be performing well, containing negligible overall biases and showing better distributions than the raw model throughout this range. For higher wind speeds, an increasingly severe bias is present, though corrections continue to have a positive effect. It is also worth noting here that the reliability of the QuikSCAT data at these high wind speeds is less established.

Though this representation of the data well illustrates $U_{10}$ bias characteristics as a function of $U_{10}$, some caution must be applied here. The effect of observational error is not accounted for is this analysis. Some of the observed features, specifically over estimation of low wind speeds and underestimation of high wind speeds, may be partially attributable to this simplification (see for example Freilich (1997) and Tolman (1998a)). However, it is clear from scatter plots (e.g. Figure 3.4 for uncorrected ACCESS $U_{10}$ ), that this spurious effect is not dominant, with the model clearly under-predicting high wind speeds relative to observations. This is not a surprising result for a model of this resolution (e.g. Cavaleri and Bertotti, 2006). These high wind speeds occur relatively infrequently over the globe (as seen in the PDFs of Figure 5.15), however, the wave generating potential of winds of this strength make such underestimations relevant here. This is discussed further in Chapter 6 .

The use of higher order statistical functions for wind corrections could be used to better correct these high wind speeds. The use of percentile based corrections, commonly used in climate downscaling studies (e.g. Hemer et al., 2011) could also be applied here, however, the limited data available at each grid point within the learning window may preclude this approach. Further investigation is left here for further work.

\subsection{Conclusions}

In this chapter, a number of techniques for statistically correcting the wind speed have been explored, based on comparisons against QuikSCAT observations. The simplest of these examined the application of a fixed correction over the entire global domain. While such corrections produced overall improvements, they were unable to adequately account for regional variation in $U_{10}$ bias over the globe. These regional biases were effectively removed with a spatially varying correction, 

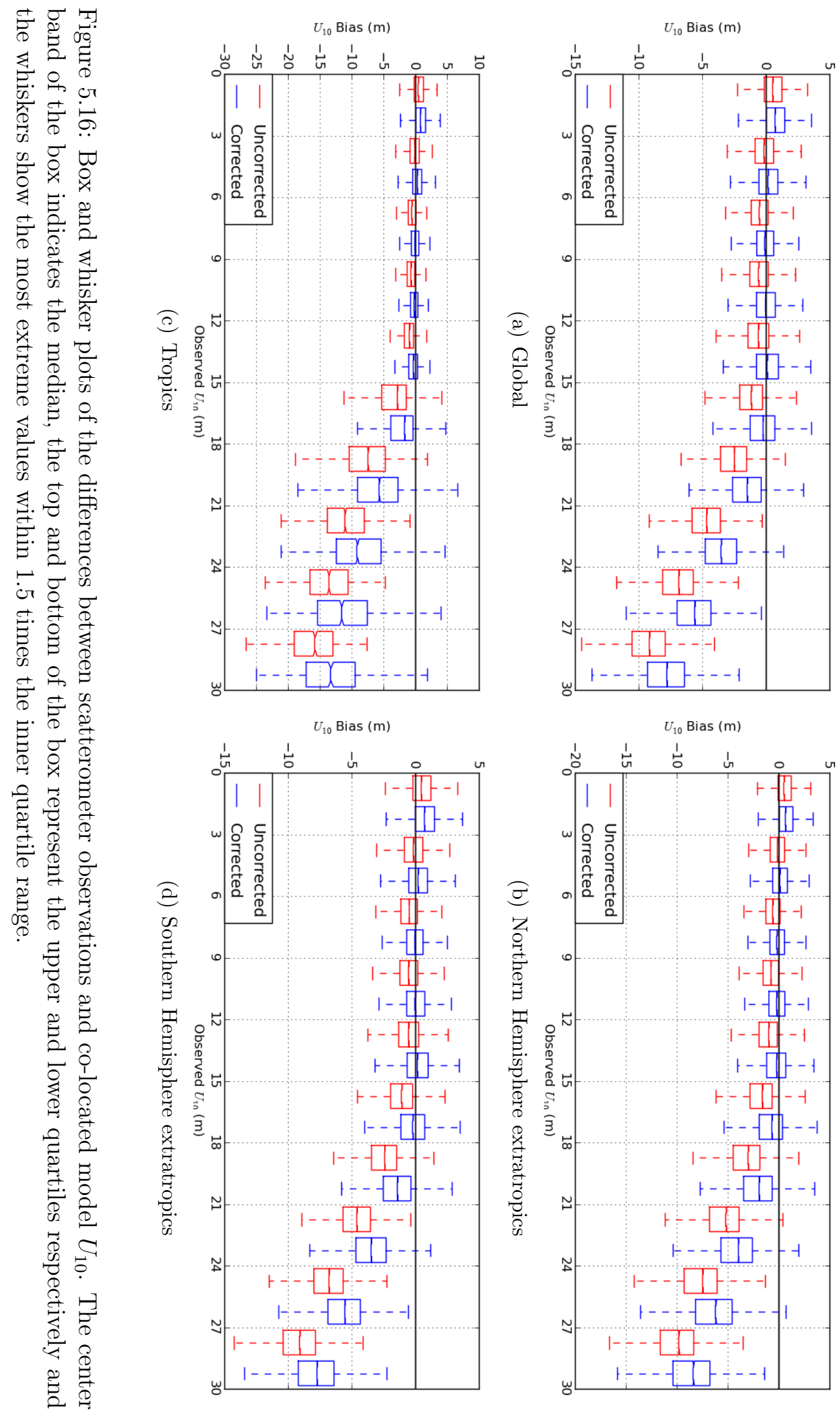
calculated independently for each model grid point, retrospectively for the entire period.

These spatially varying corrections were then extended to vary in time. A method was proposed whereby automatically evolving corrections are calculated in real time from a moving window of historical comparisons between observations and preceding forecasts. A number of spatial and temporal learning windows were explored, with a 30 day learning window, calculated individually for each model grid point was found to give the best improvement in terms of model RMSE. This relatively long learning period effectively targeted the removal of synoptic scale systematic biases, while applying independent corrections at each model grid point enabled the removal of persistent biases on fine spatial scales, such as those present along coastlines and in the lee of islands. This technique addresses the need for geographically varying corrections, as well as eliminating the need to monitor and manually adjust these corrections with time. Correcting the winds in this way, has resulted in a reduction in $U_{10}$ RMSE of about $8 \%$ and regional biases in the winds have been effectively removed.

The evolution of the systematic model bias over the four months examined here is not dramatic. Over longer periods, bias could be expected to vary seasonally, or with changes due to long lived modes of atmospheric circulation such as El Niño Southern Oscillation (ENSO). The automatically evolving correction technique presented here provides a robust means of removing such error. Similarly, it could be applied as a means of homogenising discontinuous wind field, or in the context of an evolving operational atmospheric model. Specifically, in the case of the Bureau's recently implemented ACCESS model examined here, an expected period of volatility, and planned operational changes make this particularly relevant as an operational option for maintaining consistent wind fields for downstream models. 


\section{Chapter 6}

\section{The Effect of Wind Corrections on the Wave Field}

\section{Contents}

6.1 Introduction $\ldots \ldots \ldots \ldots \ldots \ldots \ldots$

6.2 Method . . . . . . . . . . . . . . . 114

6.3 Global Sensitivity of the Wave Field to the Forcing Winds ................ 117

6.4 Results for BAJ Source Terms . . . . . . . . 120

6.4.1 Qualatitive Attribution of Large Scale Systematic Error 121

6.4.2 Quantitative Effect of Statistical Wind Corrections on the Modelled Wave Field . . . . . . . . . . . . 127

6.4.3 Residual Systematic Wave Bias . . . . . . . . . . . . . 131

6.5 Comparative Results for TC96 Source Terms . . . 141

6.6 Discussion . . . . . . . . . . . . . 146

6.6.1 Simple Source Term Tuning . . . . . . . . . . . . . . 147

6.7 Summary and Conclusions . . . . . . . . . . 152

\subsection{Introduction}

In Chapter 3 a negative bias in the ACCESS surface winds was identified. $H_{s}$ results from WW3 runs forced with these winds were found to be similarly nega- 
tively biased in Chapter 4. A number of means of correcting the ACCESS winds were explored in Chapter 5. In this chapter, the effect of these wind corrections on the modelled wave field is assessed.

Three wind fields are considered in this chapter, all based on the ACCESS winds verified in Chapter 3. The first is the uncorrected winds, the second is those to which the static homogeneous corrections determined in Section 5.4.1 have been applied. The third are those which have been corrected with spatially and temporally varying learned corrections, using a 30 day learning window, applying an independent correction at each model grid point, as determined in Section 5.4.3 and further discussed in Section 5.5.

The discussion is arranged as follows. Some background is given in Section 6.2. The response of the wave field to simple changes in the wind is then examined in Section 6.3 before examing the impact of the $U_{10}$ correctons on the modelled $H_{s}$ in Section 6.4. In Chapter 4, BAJ source terms were found to give the most consistent $H_{s}$ results over the globe, with TC96 terms producing regions of positive bias in the Southern Hemisphere, despite the negatively biased winds. As such, the bulk of the discussion centers around runs performed with BAJ terms. Some comparative discussion of TC96 terms is presented in Section 6.5. Section 6.6 contains some discussion, and a summary is given in Section 6.7.

\subsection{Method}

In discerning error sources, Rogers et al. (2005) employ the following criteria:

Test 1: If a model is forced with a wind field that contain small bias, then nontrivial bias observed in energy predictions from a wave model forced by these wind vectors implies a probable bias associated with the wave model itself.

Test 2: If a model is forced with a wind field with a bias of known sign, and nontrivial bias of opposite sign is observed in energy predictions from a wave model forced by this wind field, this implies a probable bias associated with the wave model itself. (No conclusions are drawn if the bias is of the same sign.)

Test 3: If hindcasts and wave model-data comparisons are chosen such that the 
bias from numerics and resolution is small, then the nontrivial bias in the wave model itself (i.e., internal bias) is probably associated with the model source/sink term parameterizations.

The premise in which these tests are applied by Rogers et al. (2005) differ somewhat from those applied here. In that study, conclusions are drawn from comparisons at a number of point locations, namely buoys, around the U.S. coast. The authors reason that by looking at the Northern Hemisphere winter, they remain focused primarily on wind sea and young swell in these conditions, and hence are able to draw conclusions based on local wind/wave conditions. Here, the distribution of wind and wave bias is considered spatially over the whole globe, and while this approach adds to the breadth of conclusions that can be drawn, it also complicates the issue of error separation, and attribution of error becomes a non-local exercise.

As such some amendments are needed to these simple tests to meaningfully interpret the results. Though stated, test 1 was not used by Rogers et al. (2005), citing difficulty in proving small bias in the wind field, and the requirement to separately evaluate the sensitivity of the wave model to wind field bias. In this work, explicit spatial wind evaluations and corrections have been carried out in Chapters 3 and 5. By altering the bias characteristics of the winds, the sensitivity of the wave field can be assessed. A spatial analysis of the sensitivity of the $H_{s}$ field to changes in $U_{10}$ is also carried out below. The winds that have been corrected with the spatially and temporally varying learned corrections are now known to contain small bias over the whole domain, allowing this test to be confidently applied here, in a slightly amended form:

Test 1: If a model is forced with a wind field that contain small bias, then nontrivial bias observed in energy predictions from a wave model forced by these wind vectors implies a probable bias associated with the wave model itself. Such inferences cannot, however, be drawn from a simple local comparison. This test must be applied in the context of a knowledge of the influence of propagating error.

A word of caution is introduced, however, regarding test 1 as stated above. The assertion of an unbiased wind field does not imply a wind field without 
error. Random error remains, though the time scales at which the wave model responds to these errors makes this less of a concern here. What is of potential concern is error in the $U_{10}$ distribution which may still remain. The non-linear response of waves to the forcing winds requires the statement of test 1 to be somewhat softened. The $U_{10}$ distribution has been examined in the context of the wind corrections performed in Chapter 5, and though improved, they remain imperfect. This is discussed further in Section 6.4.2.

For test 2, forcing with two alternate wind fields of opposite sign is cited by Rogers et al. (2005) as a valuable means of assessment. However, wind fields from different models can be expected to have differing error characteristics in addition to bias alone. Forcing the model here with winds that are almost identical, save their local bias characteristics, allows for more certainty in the allocation of systematic $H_{s}$ error associated with each forcing. Additionally, the ability to monitor both the wind and wave bias spatially over the globe provides a powerful diagnostic tool, allowing more reliable interpretation than was possible by the site based comparisons of Rogers et al. (2005). The statement of test 2 can hence be somewhat strengthened:

Test 2: If a model is forced with a wind field with a bias of known sign, and nontrivial bias of opposite sign is observed in energy predictions from a wave model forced by this wind field, this implies a probable bias associated with the wave model itself. A bias of the same sign in both the wind and the waves is interpreted as suggestive evidence of wind bias being a contributer to the wave bias. This evidence is reinforced in areas where the reduction of the wind bias leads to a reduction of the corresponding wave bias. As with test 1, this test must be applied in the context of a knowledge of the influence of propagating error.

With respect to test 3, Rogers et al. (2005) focus on comparisons against North American buoy observations, in the Northern Hemisphere winter, thus primarily considering wind sea and young swell in these conditions, and minimising error associated with propagation. Here, global results are considered, and any error associated with propagation numerics or resolution are not easily isolated. Care has been taken, however, to minimise such error. Third order UQ numerics (see Section 4.4.2) have been used, minimising numerical error. All global runs have been performed with $1^{\circ}$ resolution, sufficient for evaluation of the large 
scale error that is the focus here. Bathymetry data has been supplemented with a coastal polygon data set in the construction of the grids (see 4.3.1), sub-grid-scale blocking has been employed to minimise the error associated with unresolved island blocking (see Section 4.4.3) and an observation based, daily updated, explicit ice edge has been included.

The ability to compare results from different source terms within the same model framework is also advantageous here. Where the origin of error is suspected to be from sources other than the physical formulation of the source terms, comparison with the same model set-up, using different source terms, can provide corroborative evidence to this effect.

Poor specification or non-specification of currents and/or air-sea temperature differences for the purposes of stability present additional sources of external error. These are not expected to have a significant impact overall (Rogers et al., 2005), however, comment is made in the text where such omissions are locally relevant.

\subsection{Global Sensitivity of the Wave Field to the Forcing Winds}

For a fully developed sea, $H_{s} \propto U_{10}^{2}$ (e.g. Cavaleri, 1994). Thus, a $10 \%$ error in the estimate of $U_{10}$ can lead to $10-20 \%$ errors in $H_{s}$. In practice, the $U_{10}$ exponent is expected to vary between 1 (short fetch limited conditions) and 2 (fully developed seas). Ardhuin et al. (2007) for example, estimate this exponent at around 1.5 in the enclosed Mediterranean Sea. This direct relation between $U_{10}$ and $H_{s}$ of course ignores propagating waves and cannot be expected to hold for the global domain. In swell dominated regions, the wave error may bear little relation to the local $U_{10}$ error. A simple examination of the sensitivity of the mean wave field to changes in the forcing winds is presented below.

Statistical wind corrections calculated in Chapter 5 suggested a $6 \%$ increase in $U_{10}$ was required to remove the overall bias. Though it was established that such an increase is insufficient to account for the spatial variation of the error, this makes a good starting point for which to examine the sensitivity of the wave field to changes in the wind. For a $6 \%$ increase in the $U_{10}$, approximate $U^{2}$ dependence for the waves would indicate an expected increase of around $12 \%$ in 
the mean $H_{s}$. The actual percentage increase in $H_{s}$ resulting from running with $6 \%$ increased winds, is shown in Figure 6.1. As expected, the smallest response occurs in fetch limited areas around coastlines. The generation regions of the mid to high latitudes show approximately $H_{s} \propto U^{2}$ dependence, with around a $12 \%$ increase. The largest growth occurs in the tropical eastern boundaries of the major ocean basins, with a maximum increase of around $15 \%$.

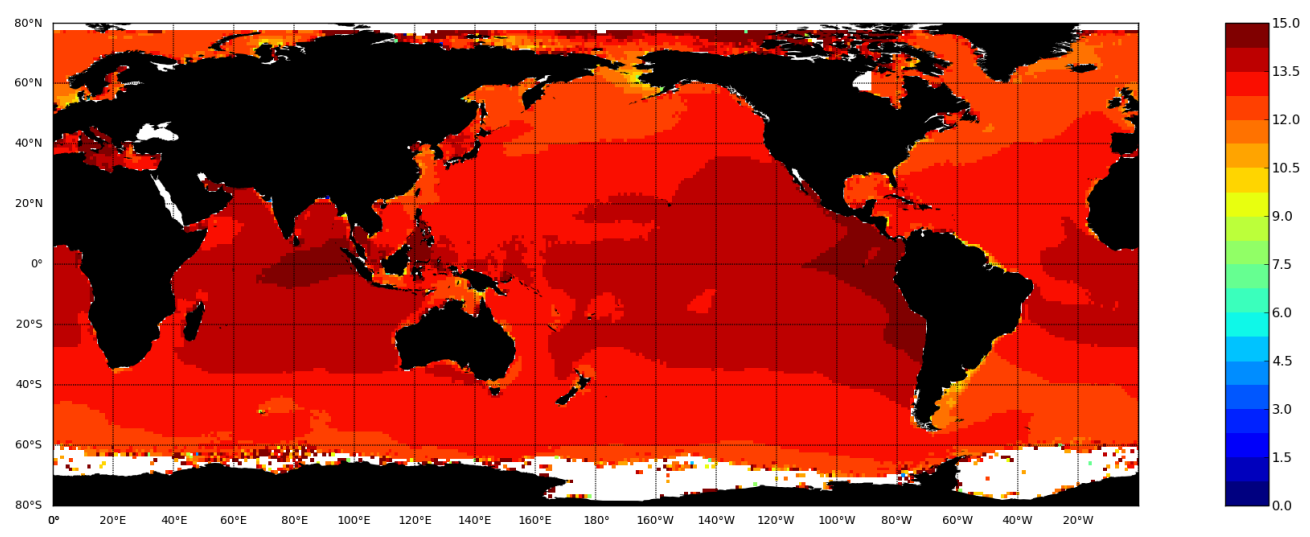

Figure 6.1: Percentage increase in the mean $H_{s}$ resulting from a $6 \%$ increase in the forcing winds.

Simply increasing $U_{10}$ everywhere gives an overall idea of the sensitivity of the $H_{s}$ field to adjustments to $U_{10}$. However, in order to better interpret the change in global $H_{s}$ bias patterns due to more complicated wind corrections, spatial sensitivity to $U_{10}$ changes in a non-local sense are of more interest here. How much of the increase in the eastern tropical Pacific is due to local wind increases, and how much to increased swell propagation from mid-latitudes for example.

To investigate this, three additional runs were performed, applying the same $6 \%$ in $U_{10}$, only restricting this increase to the Northern Hemisphere extratropics, the tropics and the Southern Hemisphere extratropics in each run respectively. Figure 6.2 shows the wave response, again shown in terms of percentage increase in mean $H_{s}$, for each case. These plots must be interpreted in the context of the wind and wave climate for this period. Mean observed $U_{10}$ and wind direction have been discussed in Chapter 3, and are shown in Figure 3.6, mean observed $H_{s}$ is shown in Figure 4.7 in Chapter 4.

For the Southern Hemisphere extratropics, increasing winds in this region only (Figure $6.2(\mathrm{c})$ ) results in virtually the same increase in the local mean $H_{s}$ as when winds are increased over the entire globe (Figure 6.1). Consistently, 


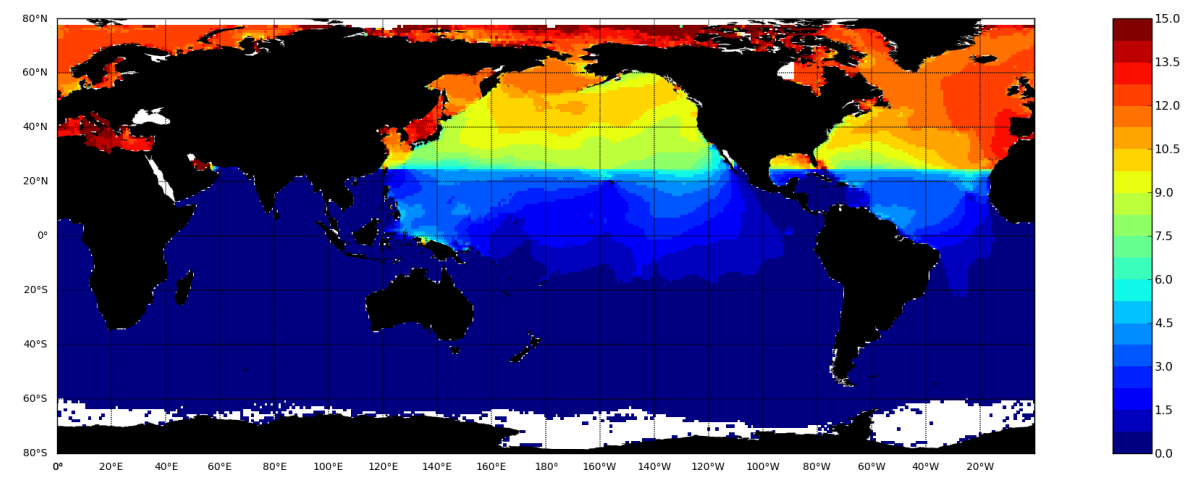

(a)
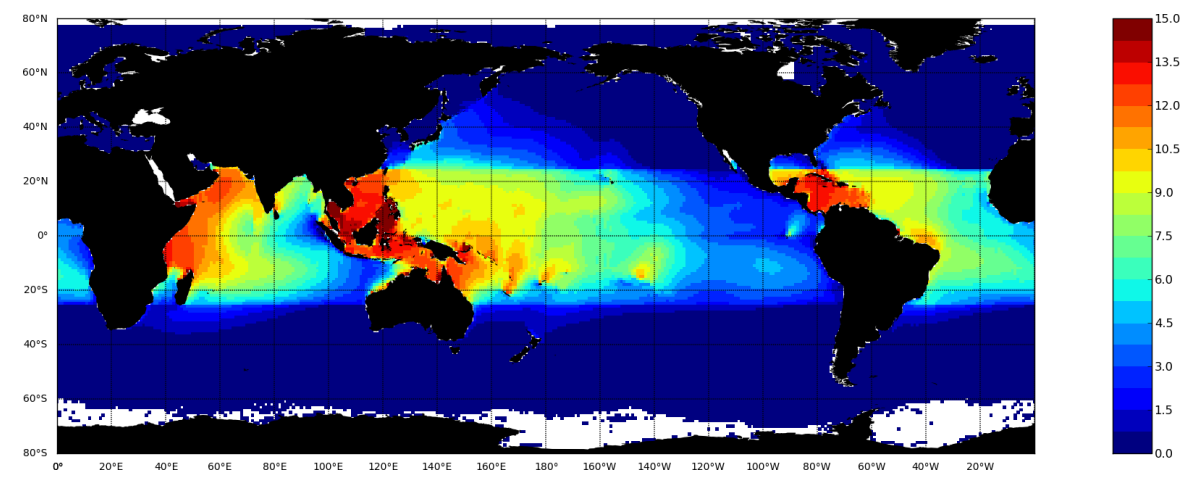

(b)

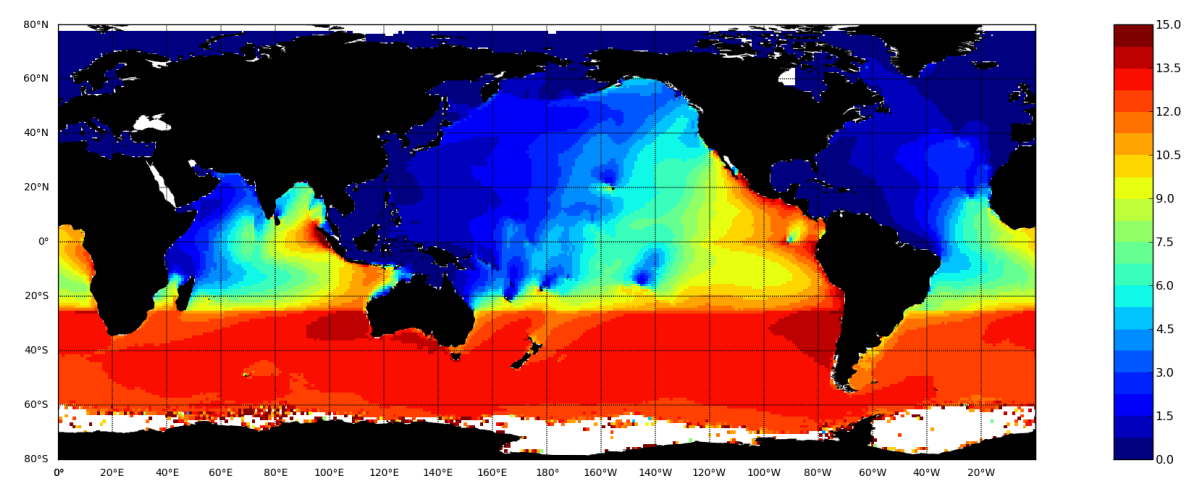

(c)

Figure 6.2: As in Figure 6.1 but with corrections applied to the Northern Hemisphere extratopics only (a) the tropics only (b) and the Southern Hemisphere extratropics only (c). 
increasing only tropical (Figure 6.2 (b)) or Northern Hemisphere extratropical (Figure $6.2(\mathrm{a})$ ) winds produces little effect on Southern Hemisphere extratropical mean $H_{s}$. This indicates that the response of the wave field to wind modifications is largely local in this region.

This is not true of either the tropics or the Northern Hemisphere extratropics. Increasing $U_{10}$ in the Southern Hemisphere results in significant increases in swell propagating right up into the north eastern Pacific. In regions dominated by swell, the relative impact of increases in the local winds is reduced. For example, increasing $U_{10}$ in the tropics only (Figure $6.2(\mathrm{~b})$ ), results in very little impact on mean $H_{s}$ in the tropical eastern boundaries of the major ocean basins.

Similar numerical simulations in this vein were conducted by Alves (2006). Breaking the global domain into 13 swell generation areas, for each region a run was performed with wind kept active only for that region. By analysing the swell propagating away from each generation region, its relative influence on global wave climate could be gauged. One of the major conclusions of that study was that swells generated in extratropical areas of the Southern Oceans spread energy throughout the entire global ocean, and are a potentially important component of the wave climate in most ocean basins in both hemispheres. It was also noted that these areas generate robust swell systems that propagate westward against the predominant storm advection direction.

Of most importance here, this illustrates the point regarding an inability to make simple local inferences about wind/wave errors. In the case of the eastern Pacific for example, the $H_{s}$ bias is more sensitive to wind biases in the Southern Ocean than it is to local wind biases. As such, the interpretation of the source of bias in the modelled $H_{s}$ field requires some care when interpreting in the spatial domain.

\subsection{Results for BAJ Source Terms}

Proceeding then to directly assess the impact of $U_{10}$ corrections on the modelled $H_{s}$, hindcasts using BAJ source terms are first examined. Results from three different forcing winds are analysed; uncorrected ACCESS winds, those corrected with static homogeneous slope corrections, and those corrected using spatially and temporally varying learned corrections. Section 6.4.1 qualitatively examines the 
broad scale attribution wind/wave error, a more quantitative assessment of the effect of $U_{10}$ corrections on the modelled $H_{s}$ results is presented in Section 6.4.2. Finally, confident that the systematic error in the forcing winds have been reduced by the spatially and temporally varying learned corrections, the remaining bias characteristics of the modelled $H_{s}$ are discussed in Section 6.4.3. For brevity, runs are referred to simply by these corrected wind labels, e.g. reference to the static homogeneous winds refers to winds that have been corrected with this method. Reference to static homogeneous waves refers to waves resulting from forcing the model with these corrected winds, etc.

The global time averaged bias of each of these wind fields, and the resulting wave field bias is shown in Figure 6.3. As in previous chapters, statistics are calculated using $1^{\circ}$ latitude-longitude boxes for $U_{10}$, and $3^{\circ}$ boxes for $H_{s}$. Presenting statistics at these scales is useful in identifying small scale features, but is somewhat extraneous for the mostly large scale focus here. Hence, contour plots of these same data are shown in Figure 6.4, with some Gaussian smoothing applied over 5 boxes in the case of the $1^{\circ} U_{10}$ bias, and 2 boxes for the $3^{\circ} H_{s}$ bias. These figures are referred to throughout this discussion.

\subsubsection{Qualatitive Attribution of Large Scale Systematic Error}

The evaluations of WW3 in Chapter 4 showed a negative bias over most of the globe in modelled $H_{s}$ when compared to altimeter data (Figures 6.3 and 6.4 (b)). Verification of the ACCESS forcing wind field against scatterometer data in Chapter 3 revealed a similar low bias (Figures 6.3 and 6.4 (a)). It seems reasonable then that the bulk of the bias in the wave field can be attributed to the forcing winds in this case.

In the context of an evaluation of WW3 under ACCESS forcing, this is a strong result. It is emphasised that the low bias in the winds for the ACCESS model have not been previously documented, and effectively answers one of the major questions of this thesis. In a more general sense, the impracticality of assessing wave model error in isolation are made clear.

However, when an unbiased wave model is forced with winds that are negatively biased, a negative bias in the waves is an expected result. The sensitivity of modelled $H_{s}$ to spatial variation in wind bias, is not easily assessed with this 


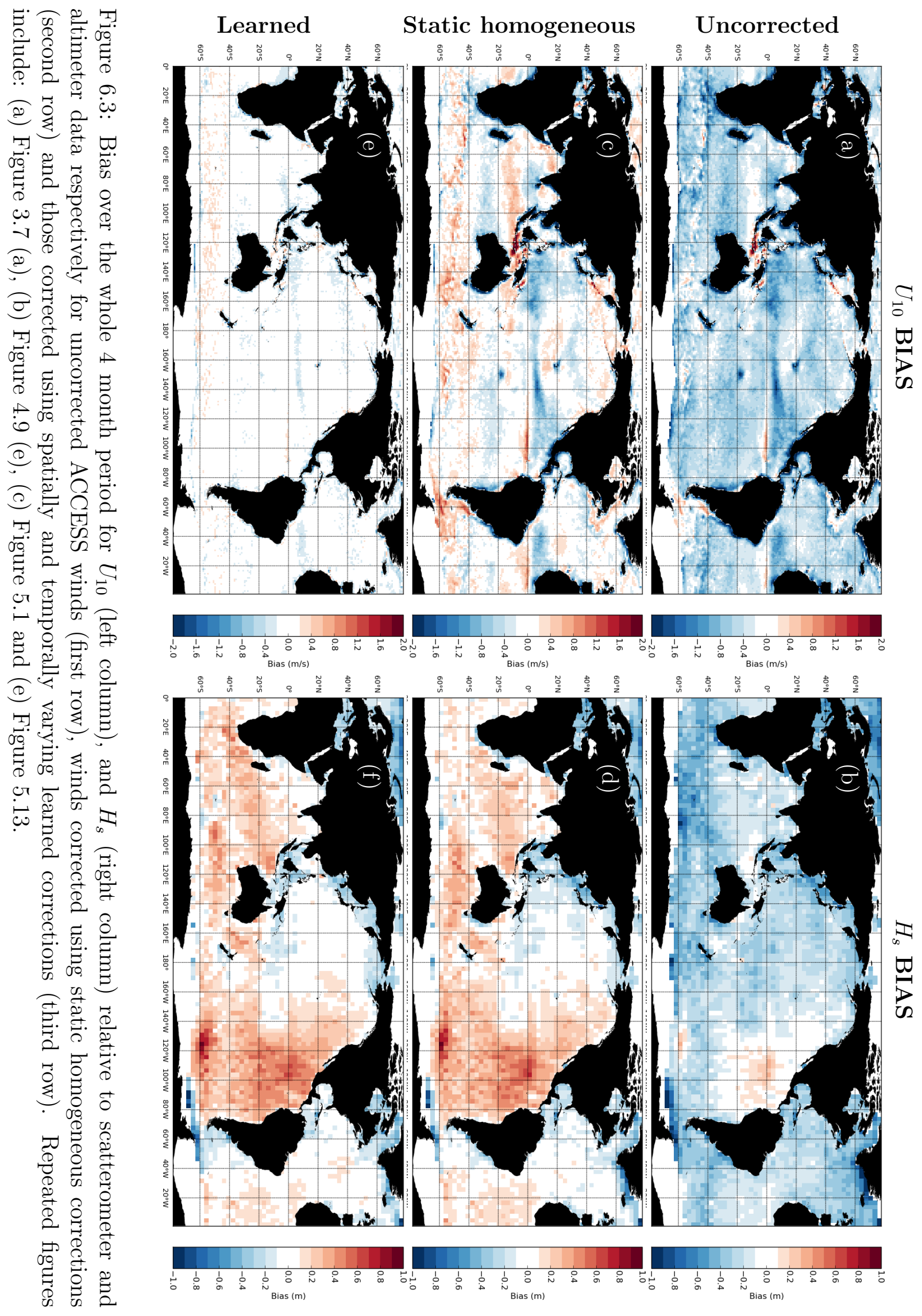




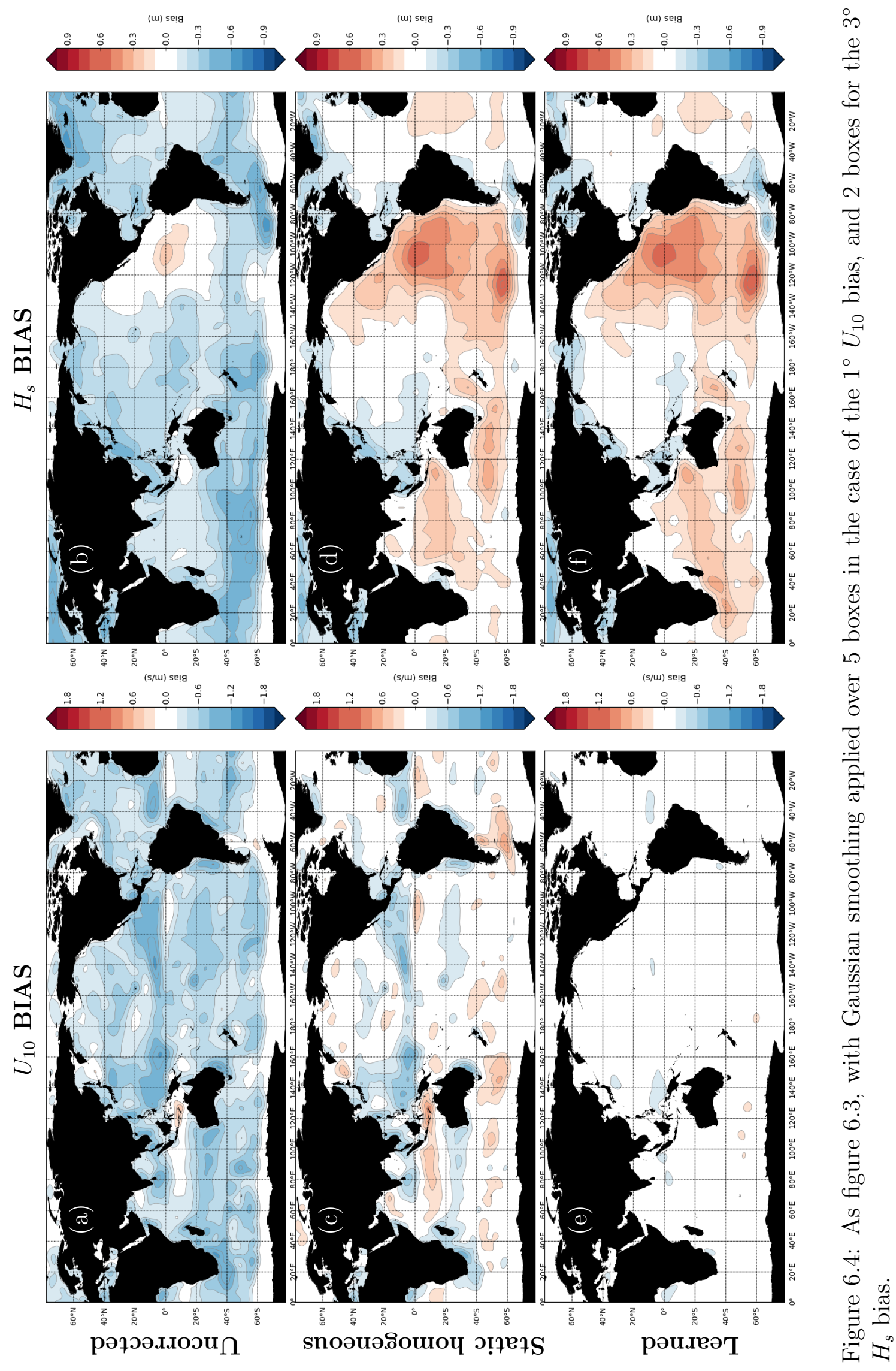


case in isolation. With the exception of the east Pacific, the wave model is biased low over the entire global domain.

By contrasting $H_{s}$ bias resulting from runs performed using the corrected winds from Chapter 5, further insight can be gained. The aim here is to examine the relative importance of $U_{10}$ bias in the $H_{s}$ bias in each case. It is reiterated that the three wind products contain similar random error, with one product being consistently negatively biased over the whole domain (Figures 6.3 and 6.4 (a)), one whose overall bias is small, but shows significant regional variation (Figures 6.3 and $6.4(\mathrm{c})$ ), and one where biases have been removed everywhere (Figures 6.3 and $6.4(\mathrm{e})$ ). This combination is useful in the application of test 2, i.e. assessing areas where the systematic wind and wave biases are of the same sign. In such areas, where the reduction or removal of the wind biases results in improved wave biases, this is interpreted as supportive evidence that the wind bias is a significant contributer to the local wave bias.

The discussion is broken up into several separate regions:

\section{Southern Ocean}

The Southern Ocean is discussed first, as the conclusions made here have some broader relevance in the overall discussion. The general under-prediction in the Southern Ocean is well explained by the low wind bias in this region for uncorrected winds. In the case of static homogeneous corrections, the positive bias in the waves in the Southern Ocean could be reasonably attributed to the corresponding positive bias in $U_{10}$. The fact that these biases remain in the case of the spatially unbiased winds, suggests that the wave model is inherently overpredicting $H_{s}$ in this region.

Within this general over-prediction in the Southern Hemisphere, a number of smaller scale features are apparent. Across all three runs, a local $H_{s}$ bias maximum can be seen in the Amundsen sea, along the Antarctic ice edge, between $100^{\circ} \mathrm{W}$ and $140^{\circ} \mathrm{W}$. A local minima is also present in the Drake Passage, south of Cape Horn in all three cases. In the case of the spatially unbiased winds, overestimated $H_{s}$ is evident off, and downstream of the Cape of Good Hope. This appears to be compensated for by regions of local, negatively biased wind speeds in both the other cases. Possible causes of these features are discussed further in Section 6.4.3 below. 


\section{Tropics}

All three runs show a positive $H_{s}$ bias in much of the eastern Pacific. In the case of both corrected wind runs the possibility of over-predicted waves correctly propagating from the South Pacific remains. The fact that this area was shown in Section 6.3 to be highly sensitive to $U_{10}$ in the Southern Ocean supports this hypothesis. However, the presence of this bias, particularly in the case of negative bias in the Southern Ocean in the case of uncorrected winds, strongly suggests a systemic issue with the wave model. A similar pattern can be seen in the east Indian Ocean. These features are clearly the result of excessive swell propagation, as discussed in Chapter 4.

Negative $H_{s}$ biases are present in the western tropical Pacific and the Gulf of Mexico corresponding with negative $U_{10}$ biases in both the uncorrected, and static homogeneous corrected cases. These $H_{s}$ biases are reduced when forced with spatially corrected winds, though low mean $H_{s}$ and $U_{10}$ in these regions (see Figures 4.7 and 3.6 respectively) increase relative observational uncertainty, requiring some caution here.

The situation in the Indian Ocean is less clear. In both the static homogeneous, and spatially and temporally varying learned cases, a positive $H_{s}$ bias associated with excess swell propagation from the Southern Ocean is present. Over-prediction around the Cape of Good Hope exaggerates this in the case of the latter. Biases in the static homogeneous winds show a lot of spatial variation, and attribution of wave bias remains difficult as waves propagate through these regions. There is, however, some suggestion of wind induced wave bias, with features such as the slight positive bias in the Arabian Sea, and a slight negative bias in the Mozambique Channel common to both wind and waves, being reduced with the spatially and temporally varying wind corrections.

In general, tropical regions that are not significantly affected by propagating errors from the Southern Ocean (as discussed in Section 6.3 above) appear to show biases that, to a large degree, reflect those of the winds.

\section{Northern Hemisphere}

With the exception of the eastern Pacific, systematic $H_{s}$ bias in the Northern Hemisphere appears to be well explained by $U_{10}$ biases. In the static homogeneous corrections case, negative $H_{s}$ biases in the western tropical Pacific, north- 
west Pacific and North Atlantic correspond to negative $U_{10}$ biases in these regions. Removal of the $U_{10}$ biases in the case of spatially and temporally varying corrections improves these $H_{s}$ biases. Once again, regions that are not affected by propagating errors from the Southern Ocean show biases that, on the large scale, reflect those of the winds.

\section{Coastal Regions}

A negative bias in the ACCESS coastal wind speeds has been previously discussed. This tendency is particularly noticeable on the Australian and South African east coasts. A corresponding negative bias in the waves can be seen in these regions. After the application of static homogeneous corrections, the coastal biases remain in the wind. Corresponding negative biases remain for $H_{s}$, despite positive bias in the neighbouring regions. The removal of these coastal wind biases in the case of the spatially and temporally varying learned corrections appears to improve $H_{s}$ bias in these areas. Though suggestive that much of these coastal $H_{s}$ biases are wind induced, the existence of strong boundary currents in these regions could also be influential. This is discussed further in Section 6.4.3.

In general, the ability to discern the spatial structure of both the wind and wave error fields is a powerful diagnostic tool. However, the separation of error remains difficult. The discussion above remains qualitative in as far as the specific claims made. The major conclusions are:

- For model runs forced with uncorrected ACCESS winds, the majority of the negative bias can be attributed to a low bias in the forcing, a result that emphasises the importance of analysing the two in parallel.

- The reduction of systematic wind biases does not result in universal improvement in modelled $H_{s}$. In some situations, internal wave model error is compensated by error in the forcing winds.

- In a general sense, it is clear that spatial bias in the wave fields is highly sensitive to that of the forcing wind fields. This applies not only to the overall bias, but its spatial variation. 


\subsubsection{Quantitative Effect of Statistical Wind Corrections on the Modelled Wave Field}

Following from the qualitative discussion above, a more quantitative assessment is given here. Verification statistics both globally and separately for the tropics and both hemispheres (North and South of 25\%) are given in Table 6.1. Focusing initially on the static homogeneous corrections, recall from Section 5.4.1, this $6 \%$ increase in $U_{10}$ reduced overall $U_{10}$ bias to near zero and improved the RMSE by about $5 \%$ in comparison to scatterometer observations, with no change in the SI. As was the experience of Greenslade et al. (2005), the waves show a corresponding improvement, with the previous negative bias of $0.27 \mathrm{~m}$ now presenting as a slight positive bias. RMSE has been reduced by $4 \%$, though SI has degraded. For spatially and temporally varying learned corrections, improvements are surprisingly less than those for the static homogeneous corrections, despite the wind itself verifying better (Section 5.4.3). Again, overall bias has been reduced, but SI has degraded. The improvement in RMSE over uncorrected winds is just $2 \%$.

Examining regions separately however, presents a somewhat different picture. The Northern Hemisphere extratropics show impressive reductions in $H_{s}$ RMSE, with spatially and temporally varying learned corrections producing a $17 \%$ improvement, with negligible remaining bias. These corrections also produce better results than the static homogeneous corrections. $H_{s}$ RMSE has however, degraded in the tropics and the Southern Hemisphere extratropics. Previous negative biases are now positive, though are less severe. In both cases, the spatially and temporally varying learned corrections degrade wave model performance slightly more than static homogeneous corrections. This is partly due to regions of negative $U_{10}$ bias in the static homogeneous case compensating for inherently positively bias $H_{s}$, such as in the eastern tropical Pacific and the Cape of Good Hope. These features are discussed further in Section 6.4.3.

Focusing only on results from spatially and temporally varying wind corrections, this spatial variation is more clearly visualised in Figure 6.5, showing percentage improvement in $H_{s}$ RMSE for each model grid point. Significant improvement is seen in most of the Northern Hemisphere, parts of the South Atlantic and despite the now positive bias, even parts of the Southern Ocean show gains. However, in line with the discussions above, large parts of the Southern Ocean show degradation. The overall reduction in skill in the tropics seen in Table 6.1 shows large regional variation. Of most note is the large improvement in RMSE 
Table 6.1: Regional statistics for $H_{s}$ relative to altimeter data for BAJ source terms when forced with uncorrected winds as well as those corrected with a static homogeneous correction and spatially and temporally varying learned corrections. $\%$ improvement refers to \% improvement in RMSE.

\begin{tabular}{lccccc}
\hline Run & Bias (m) & RMSE (m) & SI & \% Imp. & N \\
\hline Global & & & & & \\
Uncorrected & -0.27 & 0.506 & 0.150 & & 580605 \\
Static-Homogeneous & 0.057 & 0.476 & 0.171 & 4.0 & 581926 \\
Learned & 0.070 & 0.486 & 0.174 & 2.0 & 581926 \\
\hline Northern Hemisphere Extratropics & & & & \\
Uncorrected & -0.277 & 0.449 & 0.178 & & 103791 \\
Static-Homogeneous & -0.059 & 0.378 & 0.189 & 15.8 & 103791 \\
Learned & -0.033 & 0.371 & 0.186 & 17.4 & 103791 \\
\hline Tropics & & & & & \\
Uncorrected & -0.163 & 0.33 & 0.143 & & 198969 \\
Static-Homogeneous & 0.090 & 0.346 & 0.166 & -4.8 & 198969 \\
Learned & 0.106 & 0.349 & 0.166 & -5.8 & 198969 \\
\hline Southern Hemisphere & Extratropics & & & & \\
Uncorrected & -0.235 & 0.462 & 0.134 & & 409749 \\
Static-Homogeneous & 0.126 & 0.470 & 0.153 & -1.7 & 409749 \\
Learned & 0.138 & 0.481 & 0.156 & -4.1 & 409749 \\
\hline
\end{tabular}


evident in the western tropical Pacific and dramatic degradation in the eastern tropical Pacific. This, and the similar, though less dramatic presentation in the tropical Indian Ocean are consistent with the observed biases above due to excess swell propagation.

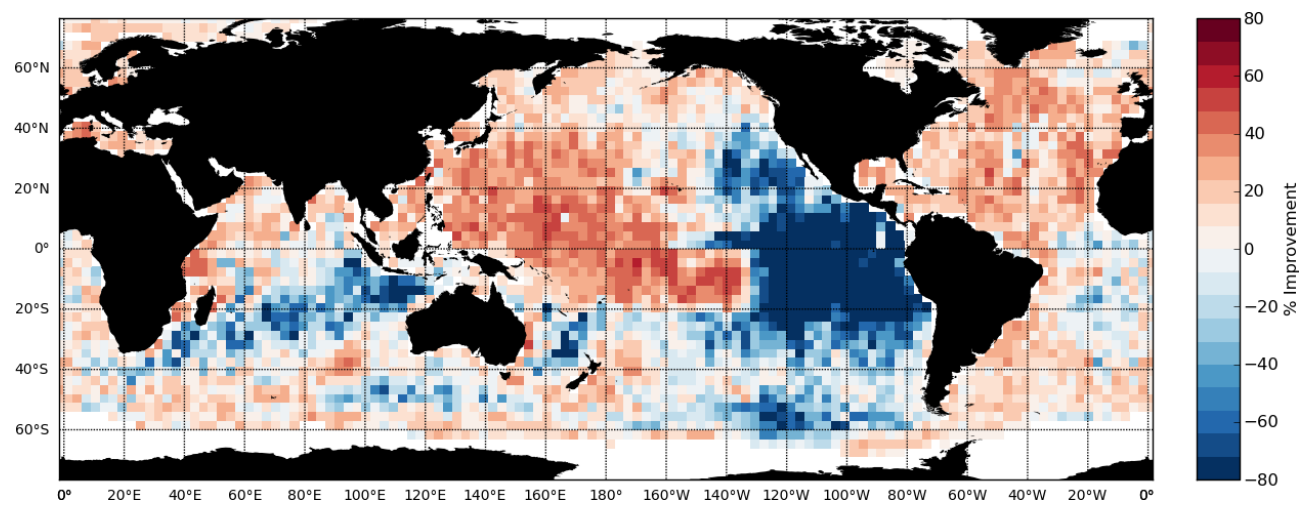

Figure 6.5: Percentage improvement in modelled $H_{s}$ RMSE achieved by correcting the forcing winds with spatially and temporally varying learned corrections using BAJ source terms.

$H_{s}$ PDFs constructed from model/altimeter co-locations are shown in Figure 6.6. These are comparable to those shown in Figure 5.15 for the winds. Observed and modelled $H_{s}$ PDFs are shown on the left hand side, on the right are error PDFs (model - observed). Results here are mixed. Over the whole globe, the distribution is better overall in the case of the corrected winds, though the peak at around $2 m$ is better captured in the uncorrected case. Higher wave heights are improved in the corrected winds cases. The error PDFs are now closer to zero centred, however the error standard deviations have been increased in all regions (the difference PDFs have been broadened).

Figure 6.7 shows box and whisker plots of the differences between modelled and observed $H_{s}$, as a function of observed $H_{s}$ (with a minimum of 100 observations required in each bin here). Again, these are comparable to the $U_{10}$ plots of 5.16. A clear overestimation in the tropics (Figure 6.7 (c)) is apparent in the range of 0-4 $m$ for corrected runs, consistent with too much swell. In the Northern Hemisphere extratropics (Figure 6.7 (b)), the corrected wind has improved the $H_{s}$ distribution throughout the wave range, with a slight over-estimation apparent in the 6-8 $m$ range. In the Southern Hemisphere extratropics (Figure 6.7 (d)), wave distributions again appear to be well captured by the corrected wind hindcast up to $6 \mathrm{~m}$. Above this, an increasing positive bias is evident, though the magnitude of the bias is less than the negative bias in this range for the uncorrected winds. 

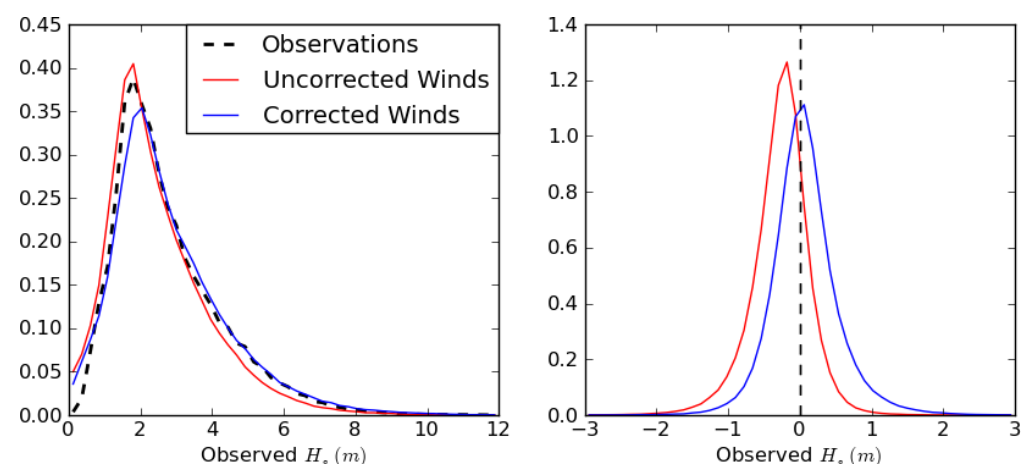

(a) Global
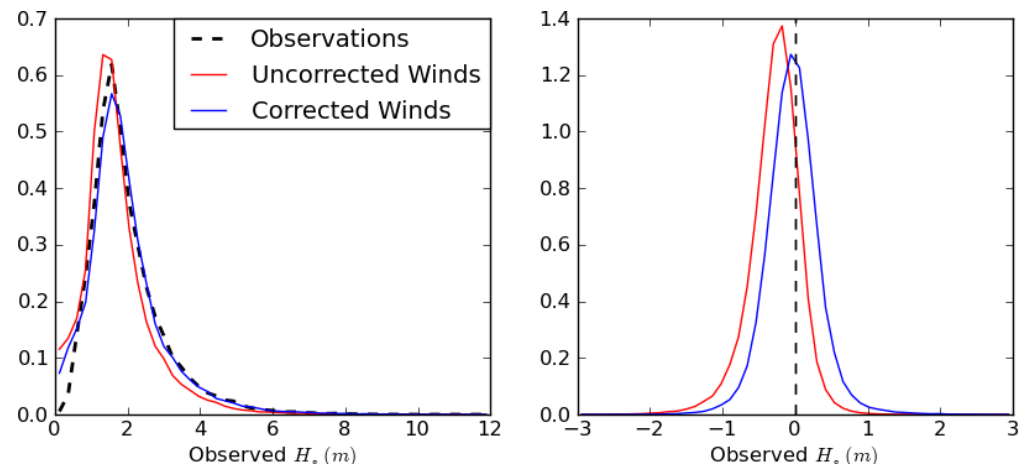

(b) Northern Hemisphere Extratropics
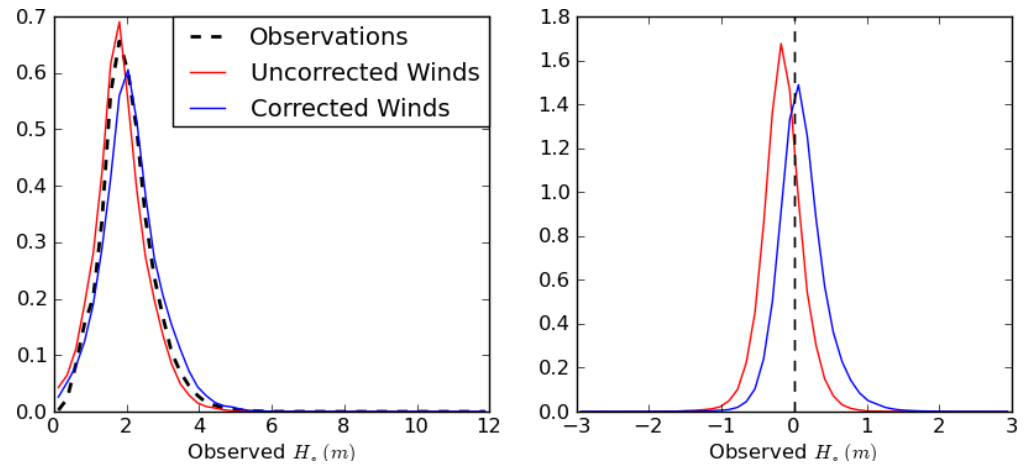

(c) Tropics
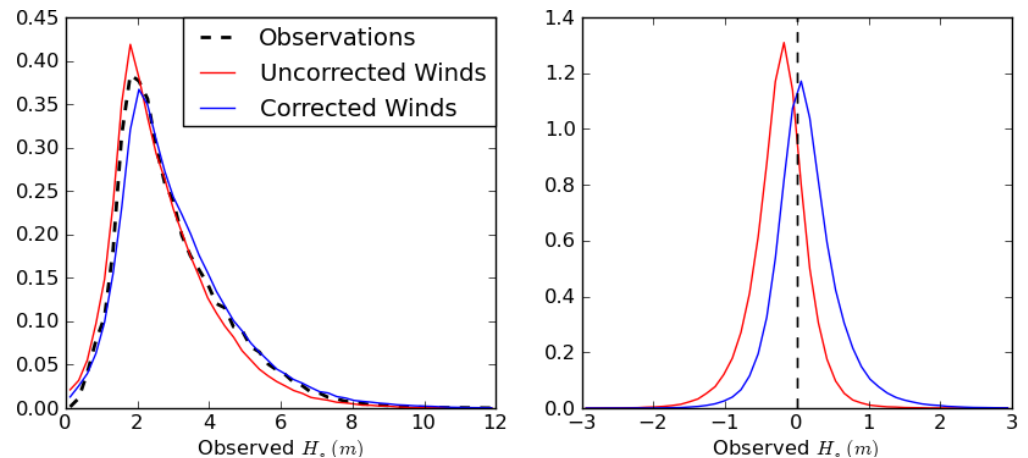

(d) Southern Hemisphere Extraptropics

Figure 6.6: PDFs of altimeter observed $H_{s}$ and co-located modelled $H_{s}$ (left) and differences (right). 
This suggests that the bulk of the positive bias in the Southern Ocean, seen in Figures 6.3 and 6.4 (f), can be attributed to these high wave heights. These over-estimations of high waves are of particular note in light of the previously mentioned spurious negative bias at these extremes expected from the neglect of observation error in this analysis. This tendency is discussed further in Section 6.4.3 below, discussing residual systematic wave error.

Improvement in the wind bias as a result of corrections is not matched in the modelled $H_{s}$ results overall. However, large regional variation is apparent, with gains in some areas offset by degradation in others. Where inherent bias in the wave model is compensated by bias in the winds, improving the forcing degrades $H_{s}$ verifications. This is most apparent in the east Pacific, where the best wave model results are achieved with uncorrected winds, where positive bias internal to the wave model is compensated by negatively biased forcing. Such compensation is easily identified here by examining the spatial distribution of the bias in both fields.

To summarise, the application of spatially and temporally varying learned corrections to the $U_{10}$ fields result in mixed results for the modelled $H_{s}$. The Northern Hemisphere shows significant improvements, while in general, results have been degraded in the Southern Hemisphere, specifically due to over-prediction of $H_{s}$ in the Southern Ocean storm tracks. This over-estimation is most prevalent for high wave heights. This then compounds the already present over-propagation of swell into the eastern Pacific and east Indian Ocean.

By correcting the wind field, a number of wave model deficiencies have been further exposed. These are discussed in the following section.

\subsubsection{Residual Systematic Wave Bias}

The results presented above have identified a number deficiencies that appear independent of the forcing winds. The discussion here focusses on features evident from the $H_{s}$ results when forced with a wind field with near zero bias, i.e. that corrected using the spatially and temporally varying learned corrections (Figures 6.3 and 6.4 (f)). By reducing wind biases to near zero everywhere in the spatial domain, remaining biases in modelled $H_{s}$ can be more confidently attributed to deficiencies in the wave model itself. The previously discussed issues surrounding propagating errors dictate some caution here however; systematic wave bias 


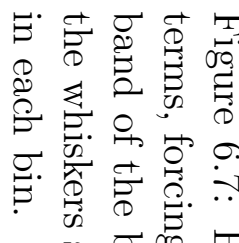
足 $\begin{gathered}0 \\ 0\end{gathered}$

导范芯

居迢:

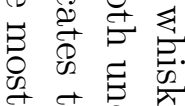

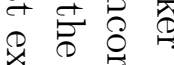

盆

०

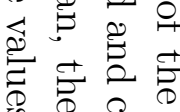

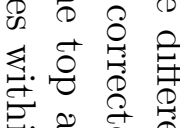

声. 胥

다 ठ

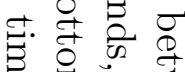

क 5 क

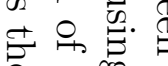

․ㅜㅇㅠ

Q

울

串

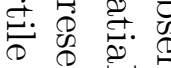

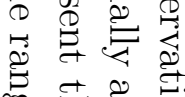

官客芑葛

$>$ 贾

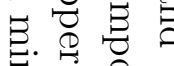

苞 气
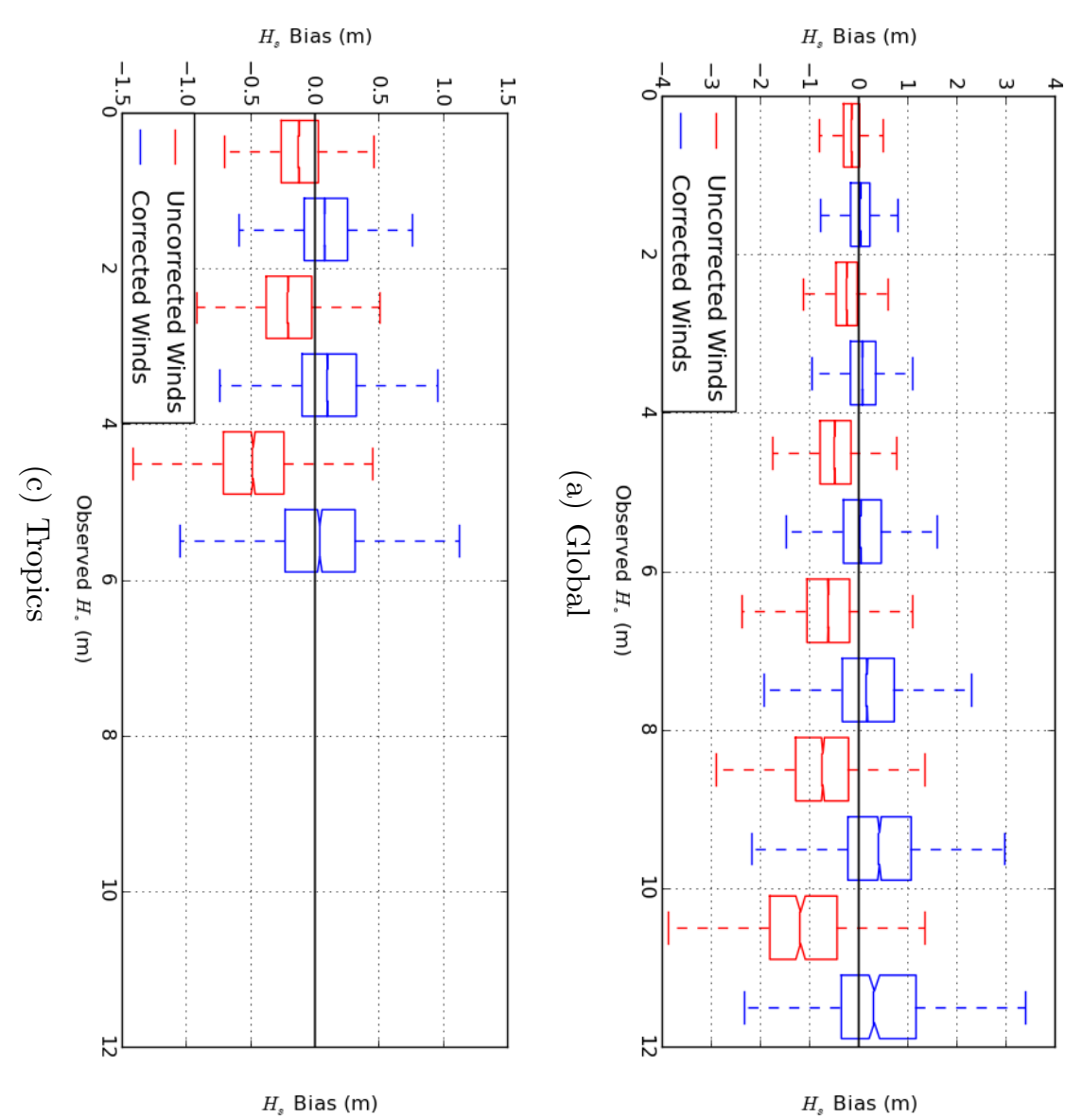

志氞党

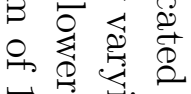

용울

ช

要过

莺. 苍

墭

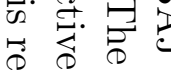

茎.
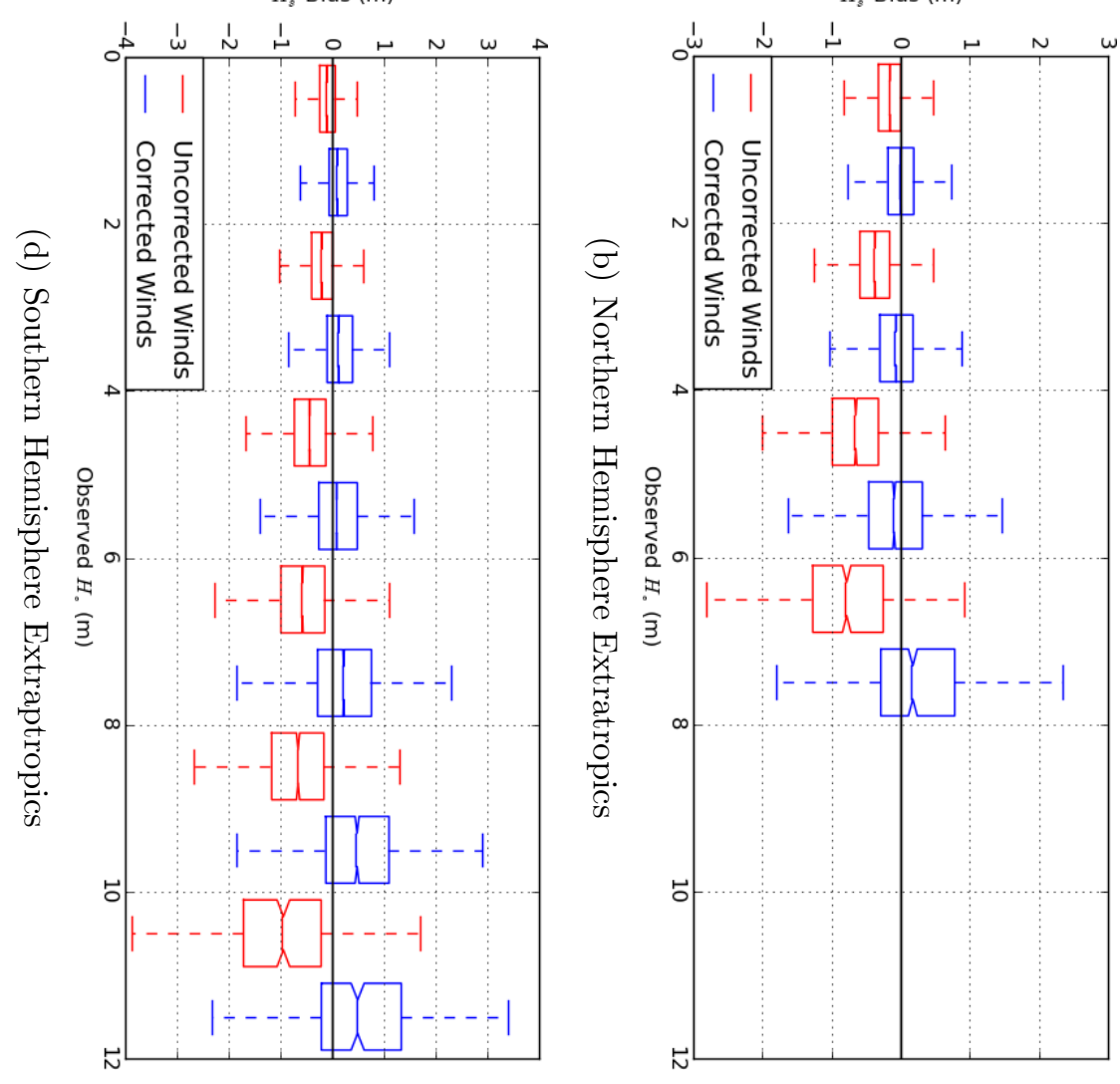
remains a non-local consideration.

\section{Over-prediction in the Southern Ocean}

It is clear that the wave model is over-predicting $H_{s}$ in the Southern Ocean when forced with unbiased winds. This raises a number of questions. Given the time of year, the wind speeds in the Southern Ocean far exceed those of the North Pacific or North Atlantic (e.g. Figure 3.6). So is this a case of the model overpredicting $H_{s}$ in strong wind conditions, or is this over-prediction unique to the Southern Ocean? This could be addressed by performing a similar analysis for the Northern Hemisphere winter months. For reasons previously discussed, i.e. a lack of winds for this period, this is not performed here. There is, however, some reason to expect that there are conditions unique to the Southern Ocean that could cause this strong positive bias.

Both the Northern and Southern mid-latitudes winters are dominated by low pressure systems. However, the continuous eastward procession of these systems in the Southern Ocean contrasts with that in the North Pacific, where their migration is far more variable (e.g. Bender, 1996). The combination of these persistent westerly winds, and the largely unbroken expanse of sea, produces potentially enormous fetches, resulting in the Southern Ocean experiencing higher wave heights for longer periods than any other body of water (e.g. Young, 1999).

Bender (1996) evaluated the WAM4 source terms for implementation in the Bureau's AUSWAM model, and found these terms to consistently over predict $H_{s}$ values when compared to buoys on the Southern Australian coast. Through a series of simulations using extremely long fetches of $20,000 \mathrm{~km}$ to represent the conditions of the Southern Ocean, he showed a significant degree of residual wave growth above the saturated PM values for high wind speeds. PM refers here to the widely used $H_{s}$ maxima for saturated conditions determined by Pierson and Moskowitz (1964), though the relatively large uncertainty in the fully developed PM values must also be acknowledged (e.g. Alves et al., 2003). Under constant $20 \mathrm{~ms}^{-1}$ winds, $H_{s}$ growth continued for several days, well above the PM predicted value of $9.86 \mathrm{~m}$. This residual growth was reportedly absent for $10 \mathrm{~ms}^{-1}$ $U_{10}$, and dramatically worse for $30 \mathrm{~ms}^{-1}$. Recent simulations using the ECMWF operational WAM model under a constant and uniform $18.45 \mathrm{~ms}^{-1}$ wind, representing a saturated PM value of slightly less than $8 \mathrm{~m}$, show $H_{s}$ still growing after several days, approaching $11 \mathrm{~m}$ (Cavaleri, 2009). This suggests that similar 
residual growth is present for the BAJ formulations used here. Problems associated with the DIA representation of the nonlinear interaction terms may also be contributing to this problem (Tolman, personal communication).

Sustained winds of this magnitude are, of course, unrealistic. However, in the dynamically evolving Southern Ocean, persistently large waves are present. A strong storm does not require days to build waves of this magnitude from a calm ocean, as in these simple fetch limited experiments. Conditions of $20 \mathrm{~ms}^{-1}$ winds with waves approaching these PM saturation limits occur with some frequency. Figure 6.8, for example, shows the percentage of time over the four months examined here that $H_{s}$ over $10 \mathrm{~m}$, and $U_{10}$ over $20 \mathrm{~ms}^{-1}$ occur simultaneously in the modelled values. In the Southern Ocean, regions where these extreme conditions are present more than $5 \%$ of the time are apparent. The modelled $U_{10}$ has also been shown to be under-predicted at these extremes (e.g. Figure 5.16) suggesting that this is an underestimation of the actual frequency of such occurrences.

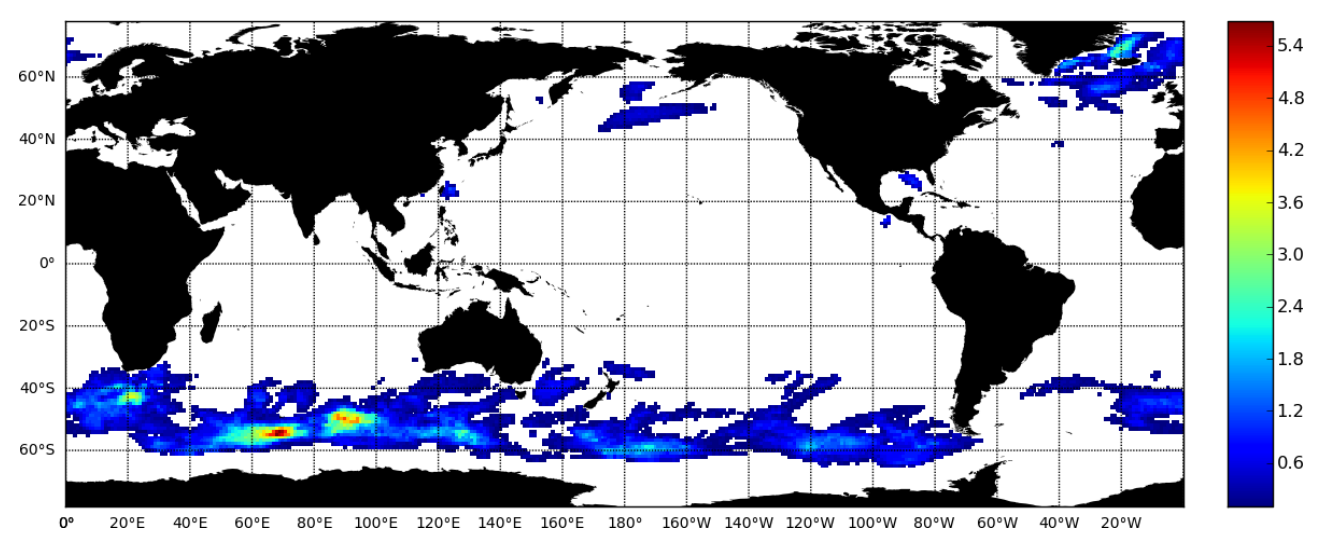

Figure 6.8: Percentage of time over the four months examined here that the modelled $H_{s}$ values over $10 \mathrm{~m}$ and $U_{10}$ over $20 \mathrm{~ms}^{-1}$ occur simultaneously.

A correlation can be seen between these areas, and the maximum biases seen in Figure 6.3 (f), as well as areas downstream of these maxima. It is clear from the box plots presented in Figure 6.7, that the positive bias in the Southern Hemisphere extratropics occurs mainly for waves above $6 \mathrm{~m}$. This suggests that the residual wave growth from the BAJ source terms at extreme values may be contributing to much of the overall positive bias in the Southern Ocean. Air-sea momentum transfer under high wind speed conditions remains poorly understood, with a lack of quality observations in these extreme conditions partly to blame. The recent work of Powell et al. (2003) and Donelan (2004) suggests that at high wind speeds, the drag saturates, or even decreases with wind speed. This 
is not currently represented in the BAJ source term formulations, and may be contributing to the positive biases at high $H_{s}$ values here. In any event, the existence of positive Southern Ocean $H_{s}$ biases, despite the fact that $U_{10}$ has been shown to be under-predicted at high wind speeds, flags this as a significant internal wave model deficiency.

An additional source of error in the Southern Ocean is offered by the recent work of Ardhuin et al. (2011), who suggest that significant wave energy is lost due to blocking by icebergs in the Southern Ocean. By including this effect in the form of a temporally varying sub-grid-scale blocking grid in WW3, they are able to reduce this bias. This may also be playing a part in the positive $H_{s}$ bias in the Southern Ocean in general, and the local bias maxima seen in the Amundsen Sea in particular.

\section{Under-Attenuation of Swell}

The second obvious feature in Figures 6.3 and 6.4 (f) is the positive $H_{s}$ bias in the eastern Pacific and to a lesser extent the tropical Indian Ocean. As discussed previously, from this case in isolation, the possibility of swell correctly propagating from an over-predicted Southern Ocean wave field exists. The fact that this east Pacific bias is present even in the case of uncorrected winds (Figures 6.3 and 6.4 (b)), where $H_{s}$ in the Southern Ocean in negatively biased, strongly suggests a systemic issue with the wave model. Indeed, this is a known deficiency in the WAM4 variant source terms. Verification studies such as Tolman (2002a) and Rogers and Wittmann (2002) first suggested the presence of too much swell in the east Pacific from these terms. It is clear from several recent studies (e.g. Ardhuin et al., 2008, 2009) that the lack of swell attenuation in the BAJ source term formulations produces unrealistic swell propagation, leading to positive biases on the eastern sides of the major ocean basins. Forcing the model with unbiased winds here simply further exposes this deficiency. This is addressed by the recent work of Ardhuin et al. (2010), who have attempted to explicitly include swell attenuation in the BAJ formulations based on observed decay rates of Collard et al. (2008). 


\section{Overestimated Variability}

From Table 6.1, the $H_{s}$ SI with corrected winds is notably worse in every region, despite the improvement seen in SI for the winds themselves (albeit modest, Table 5.4). SI over the entire domain for both uncorrected and corrected winds are presented in Figure 6.9, showing SI has degraded over the whole domain.

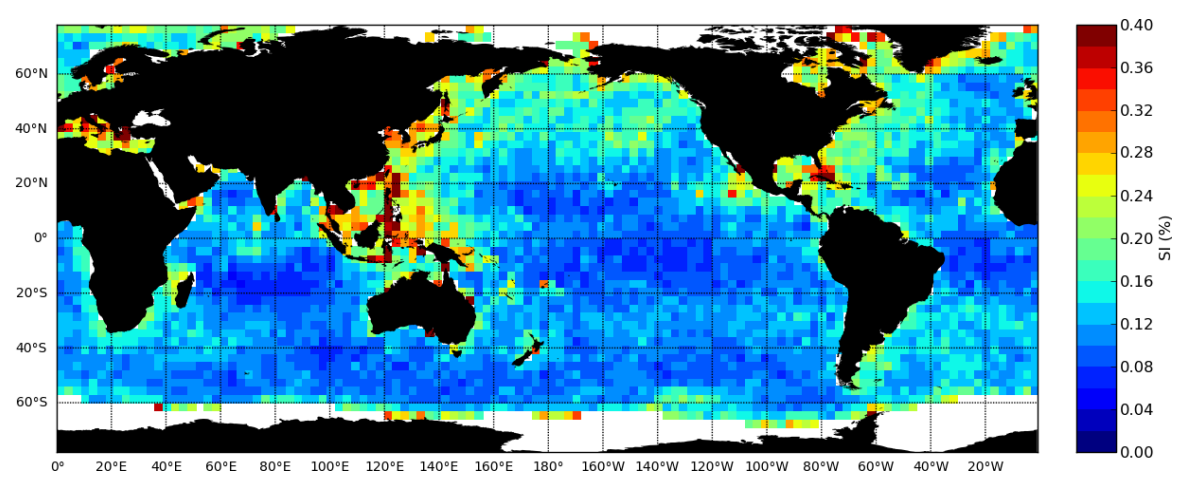

(a) Uncorrected

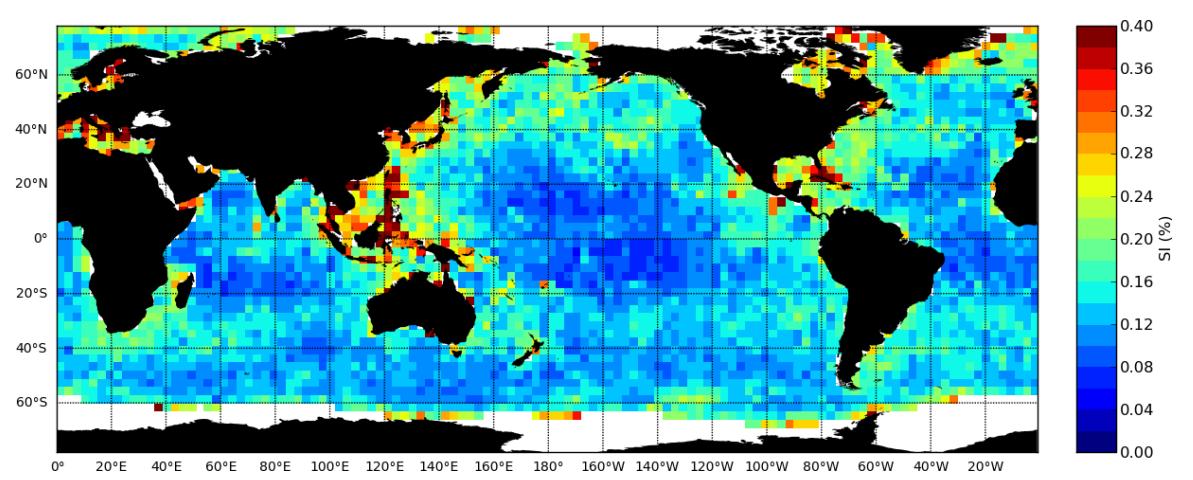

(b) Corrected

Figure 6.9: $H_{s}$ SI for BAJ runs with (a) uncorrected and (b) corrected winds. (a) is a repeat of Figure 4.9 (f)

This is examined further through consideration of Taylor diagrams. Taylor diagrams (Taylor, 2001) provide a concise statistical summary of how well patterns match each other in terms of their correlation, their root-mean-square difference, and the ratio of their variances. In order for the geometry of the diagram to work, centred RMSE must be used, i.e. that calculated after the mean of both the model and the observations have been subtracted (Note that this quantity, referred to as centred RMS by Taylor (2001) is essentially the standard deviation of the difference between model and observations): 


$$
R M S E_{\text {centred }}=\left\{\frac{1}{N} \sum_{i=1}^{N}\left(\left(M_{i}-\bar{M}\right)-\left(O_{i}-\bar{O}\right)\right)^{2}\right\}^{1 / 2}
$$

Taylor diagrams for $U_{10}$ relative to scatterometer and $H_{s}$ against altimeter data are presented in Figures 6.10 and 6.11 respectively. For each region, the centred RMSE and model standard deviation have been normalised by the observation standard deviation, allowing all regions to be plotted together. In this form, these diagrams do not contain information about biases. The model standard deviation is indicated with a dot-dash line (by definition 1 here in this normalised form). A model whose standard deviation is less than that of the observations, will appear on the origin side of this line, while one that contains too much variation will appear on the outer side of this line.

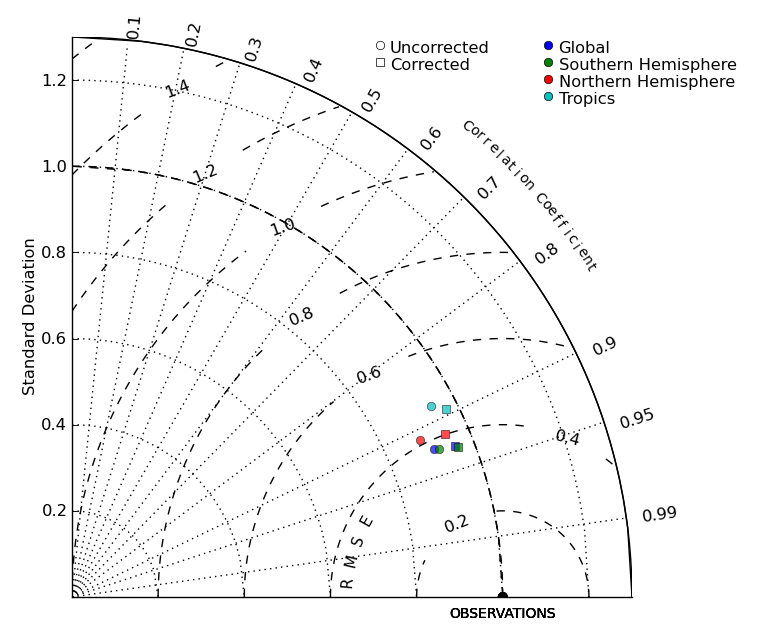

Figure 6.10: Taylor diagram showing $U_{10}$ statistics relative to QuikSCAT data for both uncorrected ACCESS winds, and those corrected using spatially and temporally varying learned corrections.

For $U_{10}$ (Figure 6.10), the model standard deviation matches that of the observations more closely as a result of the applied corrections in every region. In the case of $H_{s}$ (Figure 6.11), model variability has generally gone from being under-predicted to being over-predicted. This indicates that the wave model is exhibiting too much variability when forced with unbiased winds. This is most prevalent in the tropics, consistent with too much swell entering the region, as discussed above.

Corrected winds show good agreement with scatterometer observations in terms of bias and slightly underestimate variability. The wave model forced with 


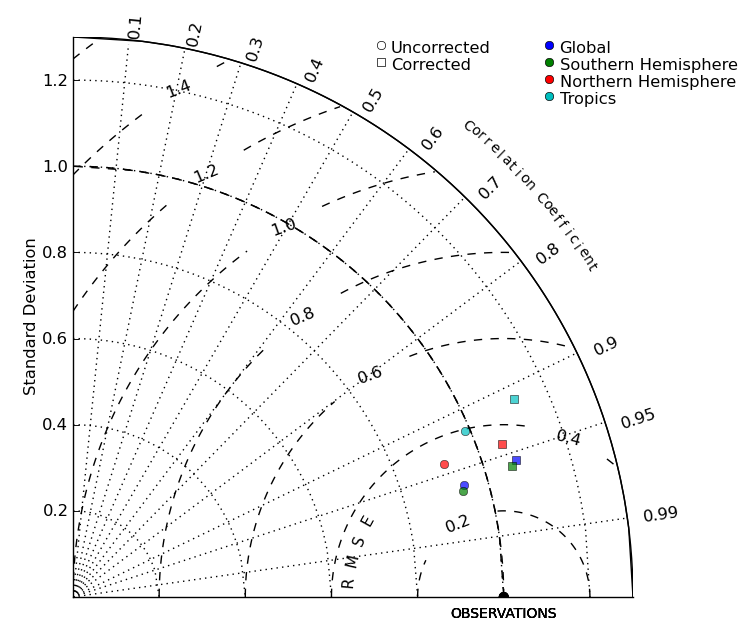

Figure 6.11: Taylor diagram showing $H_{s}$ statistics relative to altimeter data when forced with both uncorrected ACCESS winds, and those corrected using spatially and temporally varying learned corrections.

these winds produces waves with too much variability. The conclusion could be drawn that the wave model inherently produces too much variability in the $H_{s}$ field. A point of caution is raised here however. In the case of $H_{s}$, in order to match the spatial scales of variability between model and observations, 'superobbing' has been performed on the altimeter data, consisting of $1^{\circ}$ along track averages of 15-20 individual observations (discussed in Section 4.3). No such averaging was done in the case of the scatterometer observations, with this 25 $\mathrm{km}$ resolution data being directly compared to model data at $1^{\circ}$ resolution. This difference is not expected to affect bias results, however, the importance of spatial scales of representativeness on conclusions made here regarding variability are unclear. This is left for further work.

\section{Ocean Surface Current Considerations}

The effects of currents on the wave field have been ignored in the model simulations carried out in this work. Over most of the ocean, these are negligible, however, more scrutiny is required in areas of strong, persistent currents.

On the scales considered here, two current related effects are of relevance: wave-current interactions and the correct estimation of the true wind speed with respect to the moving sea surface. The relative contribution of the inclusion of each effect is quantified in the preliminary work of Hersbach and Bidlot (2008). 
In Chapter 3, the effect of ocean currents on scatterometer wind retrievals was discussed. In particular, it was noted that the wind stress is associated with the vector difference between the surface wind speed and the movement of the ocean surface, i.e. the scatterometer is measuring $U_{10}$ relative to the moving surface, while the NWP model estimates $U_{10}$ relative to a fixed frame of reference. By correcting the modelled wind speeds according to scatterometer data, the effects of currents on the wind speed felt by the waves is crudely accounted for in the mean. However, currents are dynamic. The wind correction method applied here relies on the previous 30 days data, and it is the effect of the mean current over these preceding 30 days that is applied at any given model time. If currents are indeed having a significant effect on wind corrections, and those currents show large variation on shorter than monthly timescales, this may present a significant shortcoming of the applied method.

Consider, for example, the Agulhas return current off the Cape of Good Hope. In the case of the spatially and temporally varying learned corrections, where $U_{10}$ in this region now agrees well with scatterometer observations, the waves are over-estimated (Figures 6.3 and $6.4(\mathrm{e}, \mathrm{f})$ ), both here and downstream. The neglect of wave-current interactions could be expected to produce such a result. Hersbach and Bidlot (2008) demonstrate a significant reduction in mean $H_{s}$ in this region when the effects of currents on wave advection are included. Wave steepening, leading to increase dissipation, could also be expected to reduce mean $H_{s}$. The effect of meanders of this current on the wind corrections method remains a significant question mark however.

Similar issues arise in the Drake Passage, as the Antarctic Circumpolar Current (ACC) flows between the southern tip of South America and the Antarctic Peninsula. Unlike the Agulhas current, the ACC and the wind in this region are, on average, aligned. This results in scatterometer measured wind speeds that are lower than absolute wind speeds. This could explain the local positive maxima visible in the $U_{10}$ bias for both the uncorrected, and the static homogeneous corrected winds (Figures 6.3 and $6.4(\mathrm{a}, \mathrm{c})$ ). Where wind is corrected according to the local scatterometer data (Figures 6.3 and $6.4(\mathrm{e})$ ), a low $H_{s}$ bias results (Figures 6.3 and 6.3 (f)). Again it is unclear if the wind is being mis-corrected here, or this is simply the result of a lack of wave-current interactions in the wave model.

In general, the role of currents, both in terms of their contribution to the 
modelled $H_{s}$ biases, and the potential contaminating effects on the wind speed correction methodology are appear to be significant. Further work is needed here.

\section{Atmospheric Stability Considerations}

The issue of stability on scatterometer wind retrievals has been previously discussed in Chapter 3, namely that wind retrievals are calibrated to the equivalent neutral-stability wind at a reference height of $10 \mathrm{~m}$ above the sea surface, while NWP products are estimates of the actual $10 \mathrm{~m}$ wind. Not accounting for this difference, though commonplace and justifiable overall, results in local bias features due to SST fronts. These can be seen in the south Indian Ocean and the North Atlantic, for example, in Figures 6.4 and 6.3 (a),(c) and (d) (discussed in Section 3.5).

It is well established that in unstable conditions, wind wave growth is enhanced (e.g. Cavaleri, 1994; Young, 1998). Both BAJ and TC96 terms have the ability to account for stability effects on wave growth (Abdalla and Bidlot (2002) and Tolman (2002a) respectively), requiring the ingestion of ocean-atmosphere temperature difference grids by the model. The effect on global scales is small (e.g. Tolman, 2002a; Rogers et al., 2005), and have not been included here. The exclusion of stability effects from both the wave model and the scatterometer retrievals may have the unintended effect of offsetting each other to some degree. By correcting the wind to match the scatterometer measured $U_{10}$, stability effects are crudely accounted for in the mean. As with the currents above, daily variation in stability is not accounted for however, potentially introducing variable error. Overall, $H_{s}$ bias associated with atmospheric stability though on the large scale is expected to be small.

However, both atmospheric stability and the currents could be a factor in the $H_{s}$ biases seen on mid-latitude east coasts. Strong, warm-water, boundary currents (e.g. Gulf Stream, Kuroshio, Agulhas and East Australia currents), will likely promote atmospheric instability, enhancing wind-wave growth. As discussed in Chapter 3, the existence of coastal wind biases has been established by several studies, however, the influence of ocean currents on the assumed neutral stability scatterometer winds used here may also be contributing to the negative $U_{10}$ bias in these regions. Wind corrections based on scatterometer data could then simply be resulting in overestimated $U_{10}$, compensating for the lack of inclusion of stability effects on the wave growth. This is speculative however. 
Overall, the BAJ source terms appear to respond well to spatially and temporally varying learned wind corrections in the Northern Hemisphere. On the large scale, spatial bias in the $H_{s}$ field is greatly reduced when forced with spatially unbiased winds, and RMSE is reduced by about $17 \%$. However, results are degraded in the Southern Hemisphere and tropics, due primarily to an overestimation of large waves in the Southern Ocean storm tracks, and the under-attenuation of swell.

\subsection{Comparative Results for TC96 Source Terms}

The discussion above has focussed on runs performed using the BAJ source terms. Some comparison is made here to results from TC96 terms. The above analysis is not repeated, rather some specific differences in error characteristics associated with these two sets of source terms are discussed. Runs examined include the previously discussed TC96 source terms forced with uncorrected winds (Chapter 4), and a run performed using the spatially and temporally varying learned corrections.

Figure 6.12 shows $H_{s}$ bias and SI for TC96 source terms when run under both corrected and uncorrected winds. Similar to the BAJ case (see Figures 6.3 (b) and (f)), the negative bias has been reduced in the North Atlantic and North Pacific. The increase in the mean $H_{s}$ in the Southern Ocean has resulted in the previous small negative bias now presenting as a large positive bias over most of the Southern Ocean. This is consistent with the discussions of the inherent over-prediction in the Southern Ocean by the TC96 source terms in Chapter 4. Despite a much stronger positive bias in the South Pacific, the north-east Pacific bias is not as strong as in the BAJ case. This is due to the previously discussed better handling of swell attenuation in the TC96 terms.

Verification statistics for TC96 runs with and without wind corrections applied are presented in Table 6.2. Unlike the slight improvement seen for the BAJ terms, global results show significant degradation here. The large differences seen between the hemispheres are even greater, with the Northern Hemisphere extratropics showing almost 20\% improvement in RMSE and great reduction in the negative bias, while Southern Hemisphere extratropics have degraded by almost 

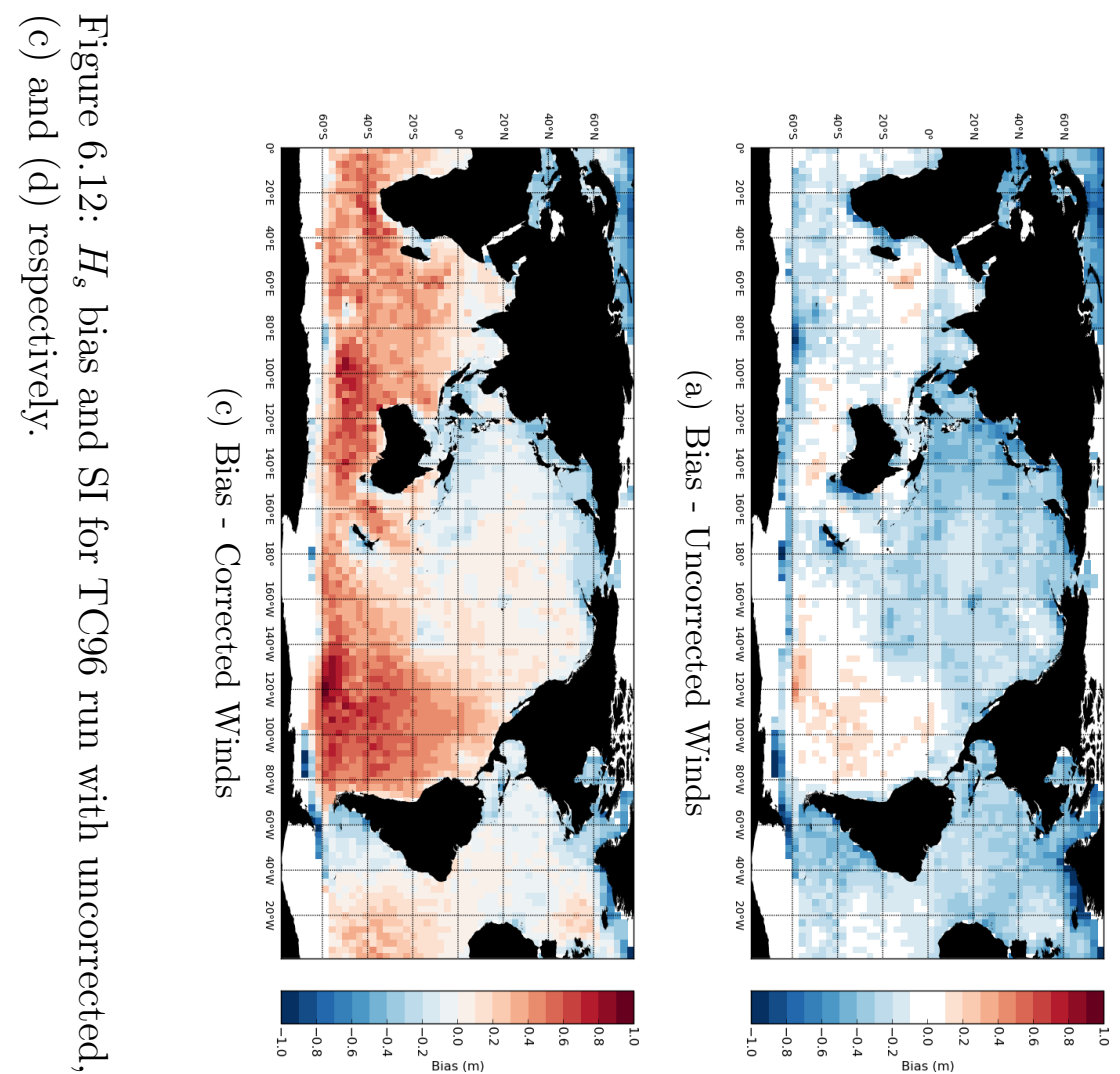

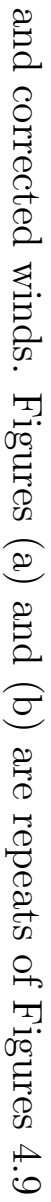
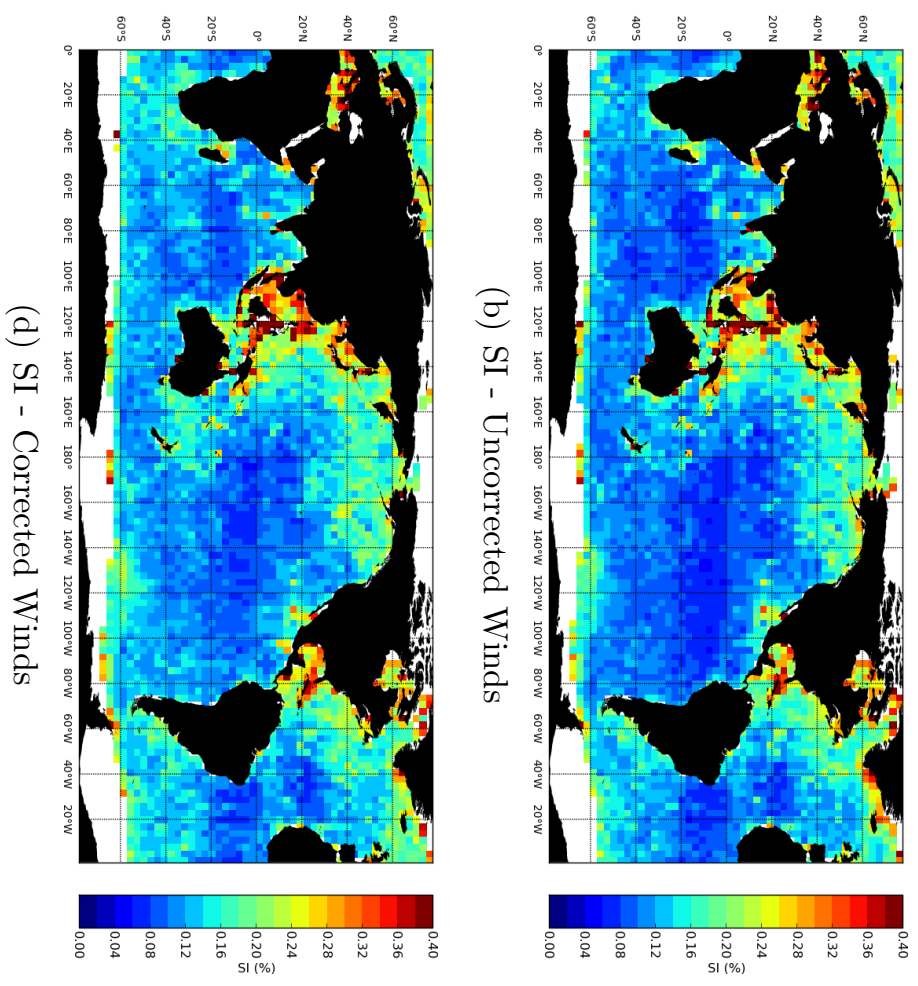
24\%. Previous negative biases have been replaced with even stronger positive ones. These differences are easily visualised when comparing the spatial percentage improvement in $H_{s}$ RMSE for TC96 terms, shown in Figure 6.13, with the corresponding BAJ results (Figure 6.5). Gains appear spatially similar in the Northern Hemisphere, with degradation appearing both more severe and more widespread in the Southern Hemisphere.

Table 6.2: Regional statistics for $H_{s}$ relative to altimeter data for TC96 source terms when forced with uncorrected winds as well as those corrected with a static homogeneous correction and spatially and temporally varying learned corrections. $\%$ improvement refers to \% improvement in RMSE.

\begin{tabular}{lccccc}
\hline Run & Bias $(\mathrm{m})$ & RMSE $(\mathrm{m})$ & SI & \% Imp. & $\mathrm{N}$ \\
\hline Global & \multicolumn{5}{c}{ R } \\
Uncorrected & -0.215 & 0.49 & 0.159 & & 580605 \\
Learned & 0.122 & 0.533 & 0.187 & -8.8 & 581926 \\
\hline Northern Hemisphere Extratropics & & & \\
Uncorrected & -0.326 & 0.485 & 0.181 & & 103791 \\
Learned & -0.089 & 0.39 & 0.192 & 19.6 & 103791 \\
\hline Tropics & & & & & \\
Uncorrected & -0.188 & 0.335 & 0.138 & & 198969 \\
Learned & 0.096 & 0.331 & 0.158 & 1.2 & 198969 \\
\hline Southern Hemisphere Extratropics & & & \\
Uncorrected & -0.147 & 0.433 & 0.137 & & 409749 \\
Learned & 0.227 & 0.535 & 0.164 & -23.6 & 409749 \\
\hline
\end{tabular}

Under unbiased winds, both BAJ and TC96 model runs share a tendency to overestimate $H_{s}$ in the Southern Ocean. However, consistent with discussions in Chapter 4, this tendency is greater for TC96. The nature of this positive bias also differs somewhat. Box and whisker plots comparable to those for BAJ of Figure 6.7 are shown in Figure 6.14 for TC96. $H_{s}$ appears to be well predicted in the Northern Hemisphere extratropics; the corrected wind has improved the $H_{s}$ distribution throughout the wave range, with the slight over-estimation seen in the BAJ terms in the 6-8 $m$ range absent here. In the Southern Hemisphere extratropics, TC96 terms exhibit overestimations for waves below $10 \mathrm{~m}$, with no bias in the 10-12 $m$ range. This is in contrast to BAJ, which show good statistical agreement with observations below $6 \mathrm{~m}$ and over-estimations above. This is likely due to the fact that the TC96 wind input term imposes a maximum allowed drag coefficient, pragmatically matching the qualitative behavior observed by Powell 


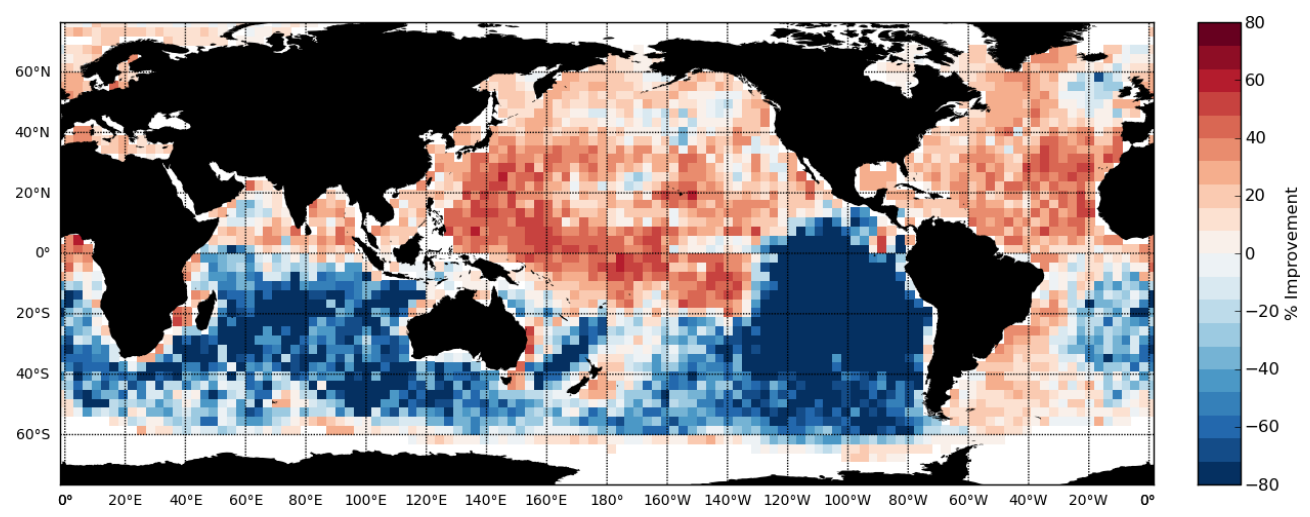

Figure 6.13: Percentage improvement in modelled $H_{s}$ RMSE achieved by correcting the forcing winds with spatially and temporally varying learned corrections using TC96 source terms.

et al. (2003) and Donelan (2004), providing more realistic input at high wind speeds (this limit is set here to $2.5 \times 10^{-3}$ ). Unlike BAJ, where the bulk of the positive bias seen in the Southern Ocean appeared to have its origin in large waves, it is in the middle $H_{s}$ ranges that this bias occurs for the TC96 terms.

$H_{s}$ SI has deteriorated over the majority of the domain (Figures 6.12 (c) and (d) and Table 6.2) as a result of the application of wind corrections. Figure 6.15 shows Taylor diagram representations for both the uncorrected, and corrected wind runs. For BAJ, all regions showed model variability going from being underpredicted to being over-predicted (Figure 6.11). Here, the TC96 terms appear to have the variability well captured in the case of uncorrected winds, at least in the extratropics, with significant overestimation apparent once the winds have been corrected.

Overall, TC96 terms appear to respond well to spatially and temporally varying wind corrections in the Northern Hemisphere, similar to the BAJ results. On the large scale, spatial bias is greatly reduced when forced with spatially corrected winds, and RMSE is reduced by almost 20\%. Again, similarly to the BAJ terms, TC96 results are degraded in the Southern Hemisphere, even more than so in the case of the latter. Where low and moderate waves appear well captured by the BAJ results, they are overestimated by TC96 terms, while TC96 produces better values for large waves. 


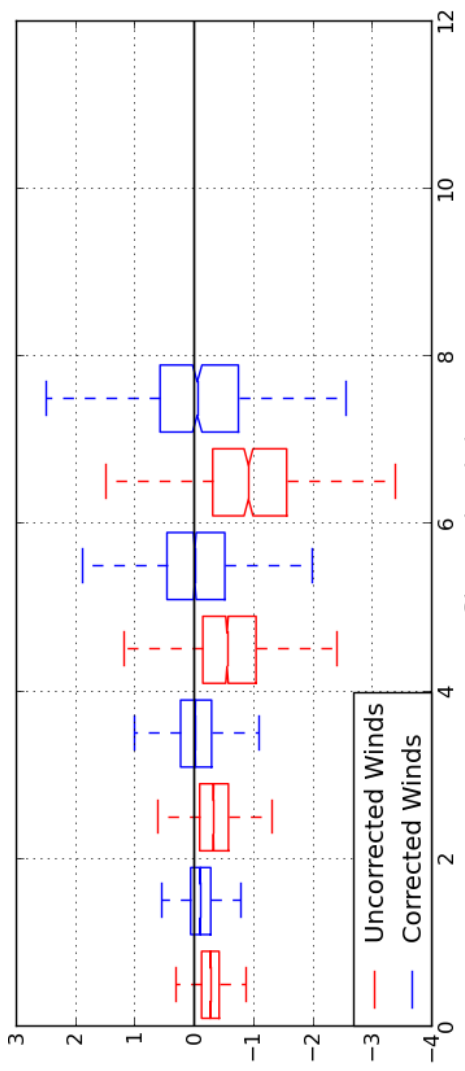

(u) se!g ${ }^{\circ} H$

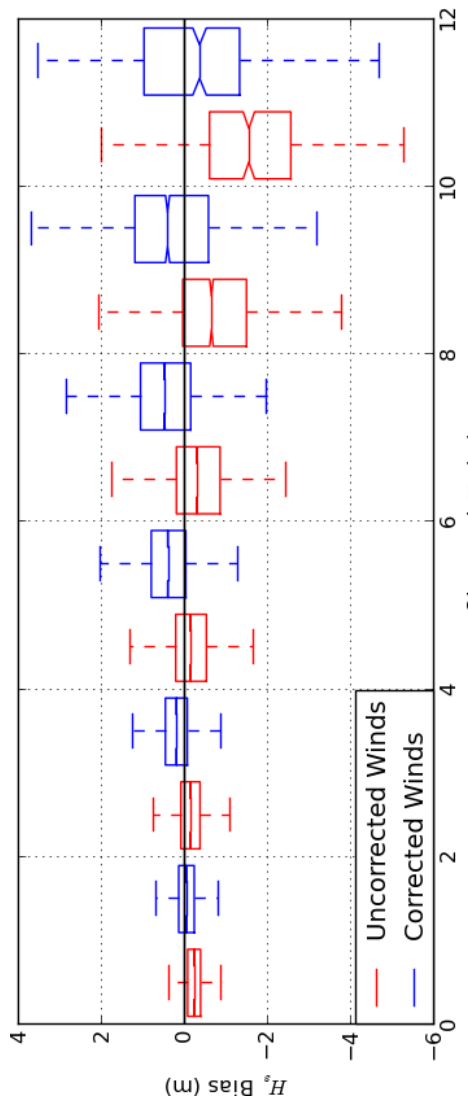

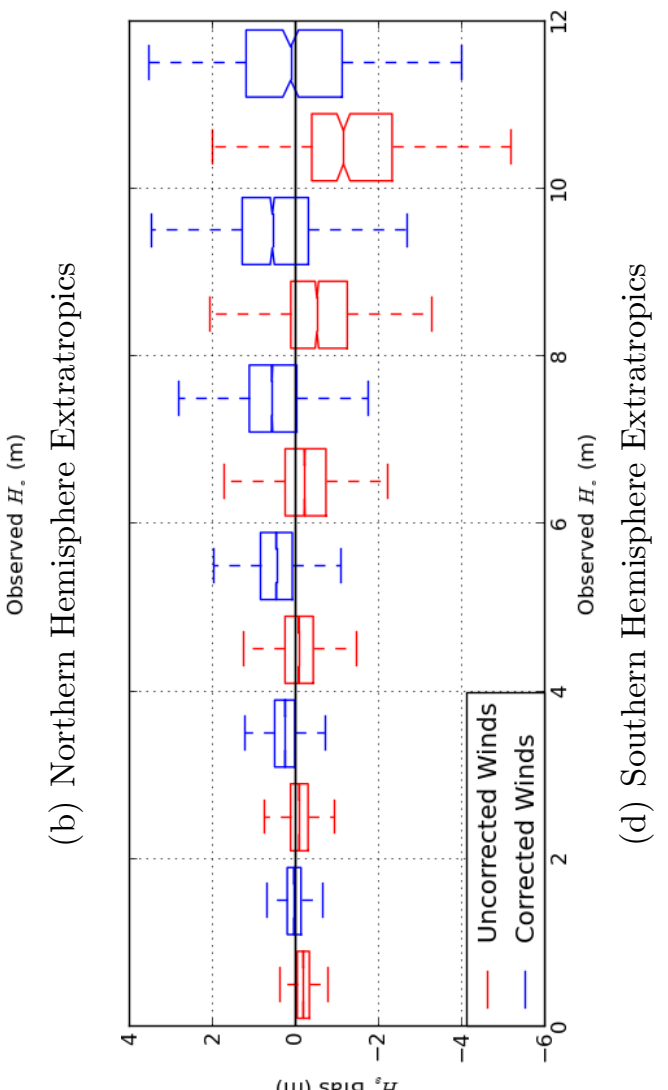

(w) se!g ${ }^{\circ} H$
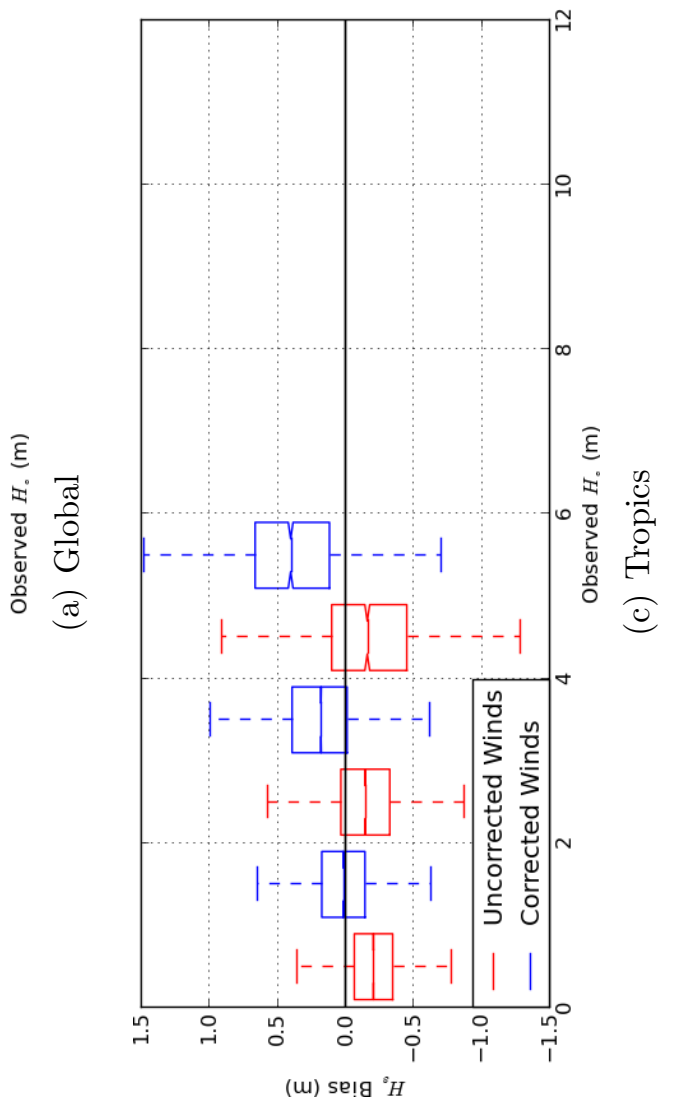


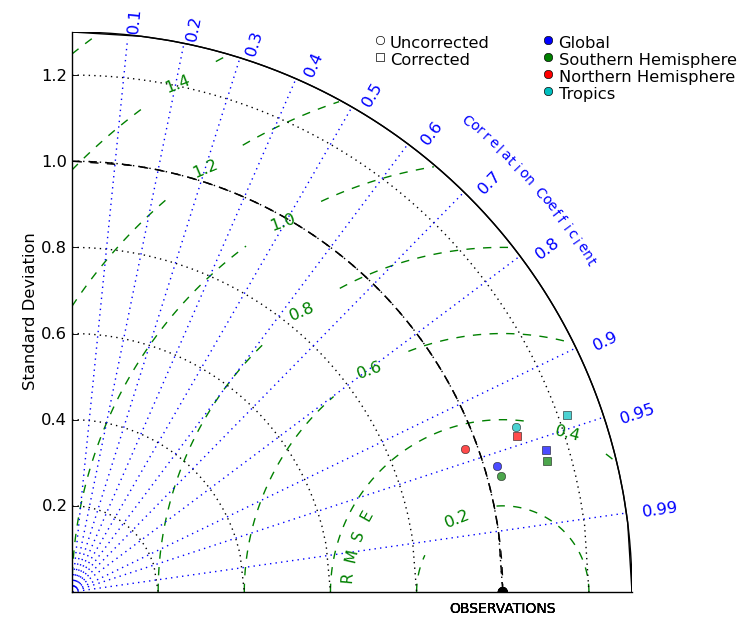

Figure 6.15: As Figure 6.11 for TC96 source terms.

\subsection{Discussion}

While it is clear that the use of spatially and temporally varying learned corrections, based on comparisons between past forecasts and scatterometer observations, provide a robust means of correcting the surface winds themselves, forcing the wave model with these winds produces mixed results in terms of modelled $H_{s}$. It is clear that greater gains are seen in the Northern Hemisphere than the Southern for both BAJ and TC96 source terms. As discussed above, this may be a seasonal effect. However, this may also be a reflection of model tuning.

The fact that modern wave model source terms, though physically based to an extent, contain significant tuning, has been discussed in Chapter 1. In the context of a review of the capability of modern wave models to properly reproduce the conditions during and at the peak of severe and extreme storms, Cavaleri (2009) make the point that model tuning, in general, is completed on the bulk of the data, with error minimisation during the most common conditions the inevitable product. Historically, buoy data have come from the major North American and European buoy networks, potentially biasing this tuning to conditions in the Northern Hemisphere.

Similarly, consideration must be made for the fact that the tuning of these terms has been done under imperfect winds. Global tuning of the BAJ source terms has been performed under ECMWF forcing winds, while TC96 terms have been tuned primarily under NCEP GFS winds. The default tunings may reflect, to some degree, the bias characteristics of these models. 
Several studies have suggested ECMWF winds are negatively biased (e.g. Chelton and Freilich, 2005; Ardhuin et al., 2007), though perhaps not as strongly as the uncorrected ACCESS winds. This work has shown that unbiased winds produce a positive bias in the Southern Ocean using BAJ source terms, which may reflect this default tuning.

NCEP GFS winds reportedly show less negative bias than the ECMWF winds (e.g. Bidlot et al., 2002; Chelton and Freilich, 2005). This is seemingly at odds with the large positive $H_{s}$ bias seen here in the Southern Ocean using TC96 terms. However, this high bias in the Southern Ocean is an issue that is common to the operational NCEP WW3 model, as previously discussed. Indeed, the existence of this bias in the NCEP WW3 model is discussed in the recent validations of Chawla et al. (2009), in which it is suggested that this may be due to changes in the operational winds since the last time the model was tuned (20002001). Specifically, since 2005, an upward shift is noted in higher wind speeds in the Southern Hemisphere, a trend that is absent in the Northern Hemisphere, manifesting in a positive $H_{s}$ bias in the former.

In general, $H_{s}$ has been shown here to be highly sensitive to changes in $U_{10}$. As such, it is unrealistic to expect a wave model to perform well under different forcing, without undertaking some re-tuning of the model. Given that such tuning of the model is necessary, the question could be asked, why not just tune to the negatively biased winds in this case? As discussed in Chapter 5, error characteristics in the forcing winds are expected to vary with time, due to factors such as seasonal changes, long lived atmospheric modes of variation, and with physical changes in the model, as illustrated in the case of the NCEP GFS winds above. By tuning the wave model to the corrected winds, the problem of maintaining this tuned state is reduced, as the automatically evolving wind corrections make future wave model retuning somewhat redundant. The ability to account for spatial variation in the systematic wind error is also something that is not easily accounted for by simple wave model tuning alone. This is briefly illustrated below.

\subsubsection{Simple Source Term Tuning}

In addition to a lack of perfect knowledge of the physics, the sensitivity of the wave model to the forcing winds requires some degree of tuning to be undertaken 
in order to achieve unbiased wave fields under a given wind forcing. Extensive tuning, either to the uncorrected ACCESS winds, or to the corrected winds is out of scope here. The following section briefly discusses some basic tuning of the wind input term in the context of the point raised above regarding spatial variation in the forcing wind bias.

The usual target for model tuning is the dissipation term representing the least physically based term in the formulations used here. However, given an $a$ priori knowledge of the errors in the forcing winds, and the focus of this study, simple tuning of the wind input term is performed here. In the case of the BAJ formulations, the wind input term takes the form (Tolman, 2009, p.25):

$$
\mathrm{S}(k, \theta)=\frac{\rho_{\alpha}}{\rho_{\omega}} \frac{\beta_{\max }}{\kappa^{2}} e^{Z} Z^{4}\left(\frac{u_{*}}{C}\right)^{2} \cos ^{p_{i n}}\left(\theta-\theta_{u}\right) \sigma N(K, \theta)
$$

where $u_{*}$ is the friction velocity, $\rho_{\alpha}$ and $\rho_{\omega}$ are the air and water densities, $\beta_{\max }$ is a non-dimensional growth parameter (constant), $\kappa$ is the von Kármán constant, and $p_{i n}$ is a constant that controls the directional distribution of $S_{i n}$. The air/water density ratio $\rho_{\alpha} / \rho_{\omega}$ is constant. $Z$ is defined as $Z=\log (\mu)$ where $\mu$ is given by Janssen (1991, eq. 16) and corrected for intermediate water depths, so that:

$$
Z=\log \left(k z_{1}\right)+\frac{\kappa}{\cos \left(\theta-\theta_{u}\right)\left(\frac{u_{*}}{C+z_{\alpha}}\right)}
$$

where $z_{1}$ is a roughness length modified by the wave-supported stress $\tau_{\omega}$, and $z_{\alpha}$ is a wave age tuning parameter. The roughness $z_{1}$, the height at which the wind is taken as zero, is defined as:

$$
\begin{aligned}
U_{10} & =\frac{u_{*}}{\kappa} \log \left(\frac{z_{u}}{z_{1}}\right) \\
z_{1} & =\alpha_{0} \frac{\tau}{\sqrt{1-\frac{\tau_{\omega}}{\tau}}}
\end{aligned}
$$

where $\tau=u_{*}^{2}$, and $z_{u}$ is the height at which the wind is specified. These two equations provide an implicit functional dependence of $u_{*}$ on $U_{10}$ and $\tau_{\omega} / \tau$.

The growth parameter $\beta_{\max }$ is set at a default value of 1.2 , as determined by Janssen (1991), such that the growth rate is in agreement with the numerical 
results obtained by Miles (1957). Adjusting this value presents perhaps the simplest means of tuning the wind input term (e.g. Ardhuin et al., 2010). However, as the growth term is dependent on $u_{*}$ which is non-linearly related to $U_{10}$, this tuning is somewhat ad-hoc. Additionally, as mentioned above, while such tunings can produce improved results overall, they can only compensate for bulk wind biases, and cannot account for any geographical variation.

To illustrate this point, some simple tuning of the $\beta_{\max }$ parameter was performed, for both the uncorrected winds, and those corrected with the spatially and temporally varying learned corrections. Within this single tuning parameter space, the simple aim of reducing the overall $H_{s}$ bias to zero resulted in $\beta_{\max }$ values of 1.6 for the uncorrected winds, and 1.13 for the corrected winds. Though undoubtedly further tuning could be done, for the purposes of this discussion, this reasonably approximates the limit of improvements that can be obtained in each case through altering this parameter alone.

$H_{s}$ validation statistics for these runs relative to altimeter data are given in Table 6.3, indicating better results by tuning to the corrected winds. At least some of this difference can be explained by the spatial $H_{s}$ biases for each case, shown in Figure 6.16. Ignoring the expected positive east Pacific bias due to under-attenuation of swell and common to both, the 'tuned' results for uncorrected and corrected winds (Figures 6.16 (b) and (c)) are compared. Despite both having an overall bias of near zero, their spatial structures differ somewhat. For the uncorrected winds case, a negative bias in the west Pacific and west Atlantic is balanced by a positive bias in the Southern Ocean. Both of these features are reduced in the corrected winds case. These features mirror signatures in the spatial $U_{10}$ biases (Figures 6.4 and 6.3 (a) and (b)). Similarly, coastal biases appear better in the corrected winds case, consistent with the corresponding local reduction of these biases in the corrected winds.

Though simplistic, this example illustrates the limitations of tuning the wave model to compensate for biases in the winds. In addition to the previously discussed need to maintain this tuning over time, such bulk tuning can not account for the spatial structure of the wind error.

Most importantly for the purposes of model development, remaining biases can more confidently be attributed to deficiencies in the wave model itself. Indeed, a number of possible explanations for the significant remaining biases present in the 'tuned' model run with corrected winds have been discussed above, e.g.: 
Table 6.3: Regional statistics for $H_{s}$ relative to altimeter data for BAJ terms with uncorrected winds and the default setting for the $\beta_{\max }$ tuning parameter (1.2), with uncorrected winds and increased $\beta_{\max }$ (1.6) and with spatially and temporally varying learned corrections applied to the wind and a reduced $\beta_{\max }$ (1.13). \% improvement refers to \% improvement in RMSE.

\begin{tabular}{lcccccc}
\hline Wind Forcing & $\beta_{\text {max }}$ & Bias & RMS & SI & \% RMSE Imp. & N \\
\hline Global & & & & & & \\
Uncorrected & 1.20 & -0.270 & 0.496 & 0.15 & & 580605 \\
Uncorrected & 1.60 & -0.000 & 0.475 & 0.172 & 4.2 & 581926 \\
Learned & 1.13 & -0.005 & 0.459 & 0.166 & 7.5 & 581926 \\
\hline Northern Hemisphere & Extratropics & & & \\
Uncorrected & 1.2 & -0.277 & 0.449 & 0.178 & & 103791 \\
Uncorrected & 1.6 & -0.096 & 0.389 & 0.19 & 13.4 & 103791 \\
Learned & 1.13 & -0.085 & 0.367 & 0.18 & 18.3 & 103791 \\
\hline Tropics & & & & & & \\
Uncorrected & 1.2 & -0.163 & 0.33 & 0.143 & & 198969 \\
Uncorrected & 1.6 & -0.011 & 0.317 & 0.158 & 3.9 & 198969 \\
Learned & 1.13 & 0.061 & 0.326 & 0.16 & 1.2 & 198969 \\
\hline Southern Hemisphere & Extratropics & & & \\
Uncorrected & 1.2 & -0.235 & 0.462 & 0.134 & 0 & 409749 \\
Uncorrected & 1.6 & 0.052 & 0.465 & 0.156 & -0.6 & 409749 \\
Learned & 1.13 & 0.057 & 0.441 & 0.148 & 4.5 & 409749 \\
\hline
\end{tabular}




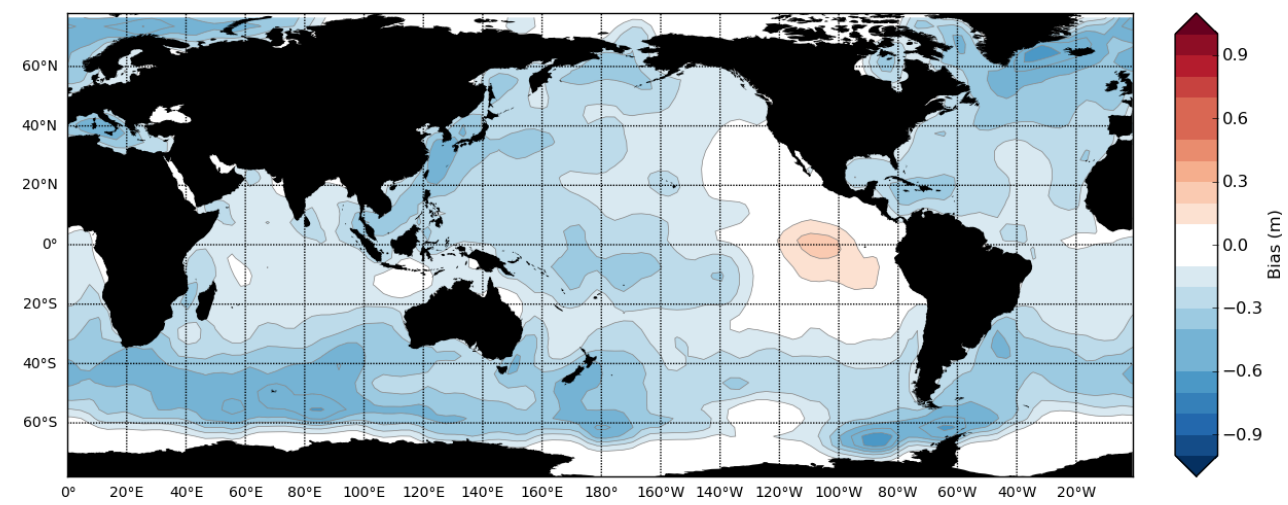

(a) Uncorrected winds, $\beta_{\max }=1.2$ (default)

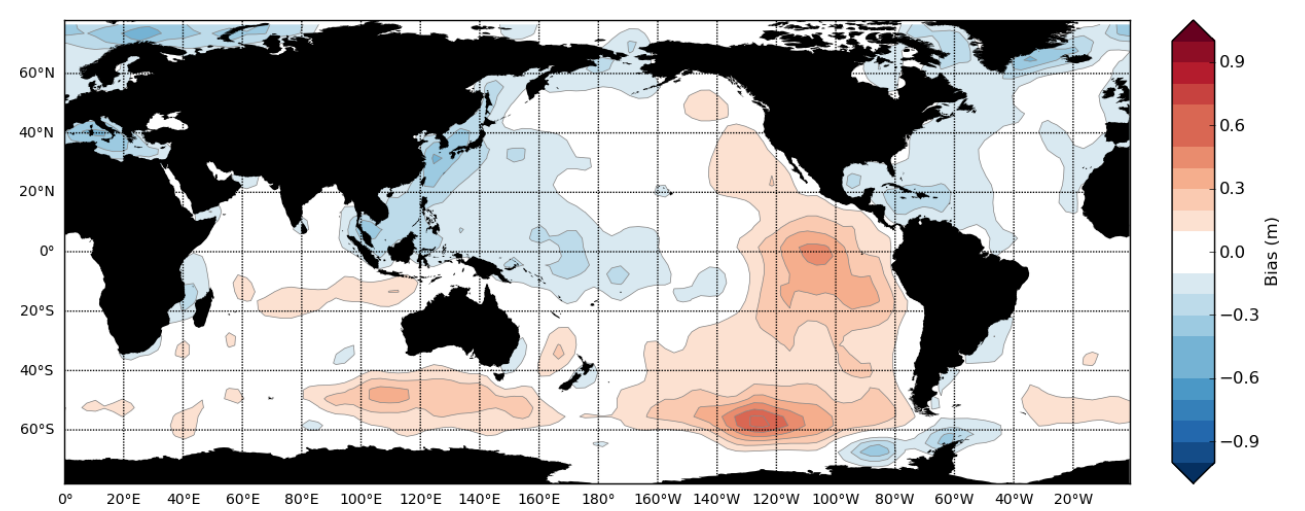

(b) Uncorrected winds, $\beta_{\max }=1.6$

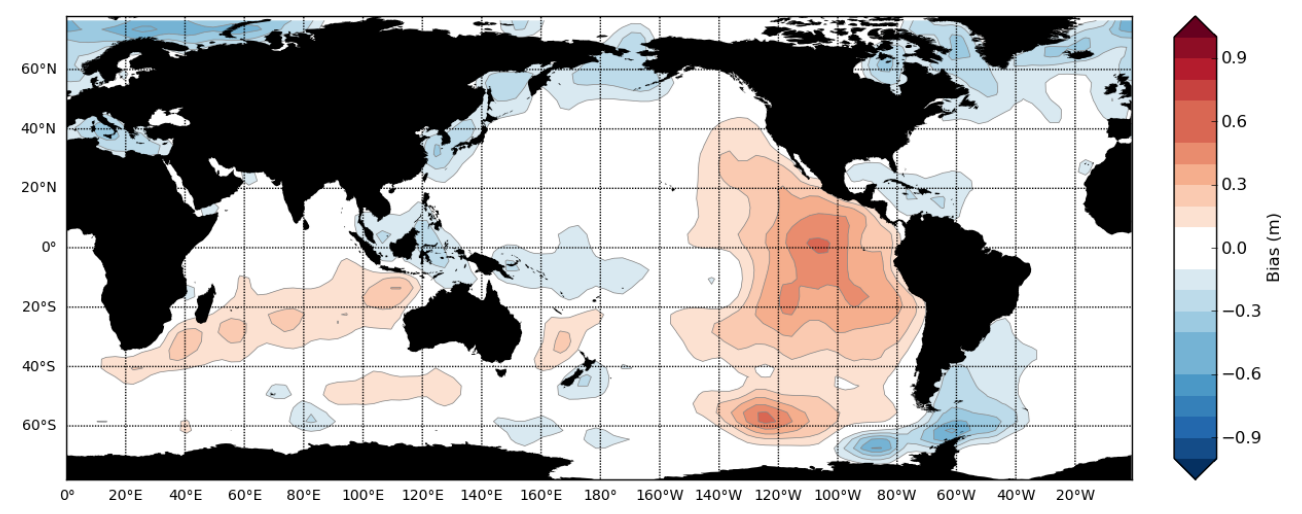

(c) Corrected winds, $\beta_{\max }=1.13$

Figure 6.16: $H_{s}$ bias relative to altimeter data for BAJ terms with (a) uncorrected winds and the default setting for the $\beta_{\max }$ tuning parameter (1.2) (repeat of Figure 6.4 (b)), (b) uncorrected winds and increased $\beta_{\max }$ (1.6) and (c) with spatially and temporally varying learned corrections applied to the wind and a reduced $\beta_{\max }(1.13)$. 
- Positive bias in the eastern Pacific Ocean, the eastern tropical Indian and the Tasman Sea can be explained by a lack of swell attenuation in the BAJ source terms.

- The positive bias to the South of the Cape of Good Hope (and contributions downstream) and the negative bias in the Drake Passage may be related to wave-current interactions.

- The positive bias to the south of Australia and in the Amundsen Sea may be due to residual growth for high wind speeds and/or blocking due to icebergs.

These explanations are speculative, and further work is required. The emphasis here is that by performing the spatially and temporally varying wind corrections applied in this work, the contribution of the systematic wind error to the $H_{s}$ bias is significantly reduced, allowing inherent wave model error to be more effectively isolated.

\subsection{Summary and Conclusions}

In this chapter, the spatial biases in the wave field resulting from three different wind fields with known spatial biases have been compared. These consist of the uncorrected ACCESS winds, and those that have been corrected with both static homogeneous corrections, and spatially and temporally varying learned corrections, as determined in Chapter 5. For runs performed using the BAJ source terms, the following conclusions have been drawn:

- Qualitative large scale error attribution

- For model runs forced with uncorrected ACCESS winds, the majority of the negative bias can be attributed to a low bias in the forcing, a result that emphasises the importance of analysing the two in parallel.

- The reduction of systematic wind biases does not result in universal improvement in modelled $H_{s}$. In some regions, internal wave model error is compensated by error in the forcing winds.

- In a general sense, it is clear that spatial bias in the wave field is highly sensitive to that of the forcing winds. This applies not only to the overall bias, but its spatial variation. 
- Quantitative effect of wind corrections on the modelled $H_{s}$

- The improvement in the wind bias as a result of corrections is not matched in the modelled $H_{s}$ results overall.

- Large regional variation is, however, apparent. Results in the Northern Hemisphere indicate strong improvements (approximately 17\% in terms of RMSE), while in general, results have been degraded in the Southern Hemisphere. The tropics also show degradation, due primarily to excess swell on the eastern boundaries of the major ocean basins.

- Small scale features such as coastal biases show improvement

- Residual wave biases when forced with corrected winds.

- A positive bias is present in the Southern Hemisphere due primarily to an overestimation of high $H_{s}$ values in the Southern Ocean storm tracks.

- A positive bias is present in the east Pacific and east Indian Ocean. This is due both to the over-prediction of waves in the Southern Ocean and the inherent lack of swell attenuation in the BAJ source terms.

- Smaller scale features are apparent, such as a positive bias off the Cape of Good Hope, and a negative bias off Cape Horn. The origin of these features is unclear. Current effects that are not included in the wave model are a possible explanation. The effect of currents on the wind correction methods applied may also be a factor.

For TC96 source terms, conclusions are similar. TC96 terms appear to respond well to spatially and temporally varying wind corrections in the Northern Hemisphere, similar to the BAJ results. On the large scale, spatial bias is greatly reduced when forced with spatially unbiased winds, and RMSE is reduced by almost 20\%. Results are degraded in the Southern Hemisphere, even more than the BAJ terms. Where low and moderate waves appear well captured in the case of BAJ, they are overestimated by TC96 terms, while TC96 produces better values at high $H_{s}$, likely due to a capping of the drag coefficient.

In the context of wave model development, the need to assess the spatial wind bias has been made clear. In general, regions where the wave model has degraded 
as a result of correcting the winds has highlighted problems in the wave model itself, which, in many cases, were masked by compensating systematic errors in the forcing winds. 


\section{Chapter 7}

\section{Summary and Conclusions}

\section{Contents}

7.1 Summary and Key Findings: . . . . . . . . 155

7.2 Further Work . . . . . . . . . . . 159

7.3 Closing Remarks . . . . . . . . . . . . 162

\subsection{Summary and Key Findings:}

This thesis has addressed the following questions:

- To what extent can the large scale systematic biases in the waves be attributed to the forcing winds?

- Do statistical adjustments to remove these winds biases lead to better wave forecasts?

- What are the residual wave biases?

In answering these questions, the following analysis has been undertaken:

\section{Altimeter Validations}

In Chapter 2, $H_{s}$ data from the Jason-1 and Envisat altimeters altimeters were validated against in situ buoy data from NDBC and MEDS buoy networks. Data covered a period of three years for Envisat and over four years for Jason-1. 
Co-location criteria of $50 \mathrm{~km}$ in space and 30 minutes in time yielded 3452 and 2157 co-locations for Jason-1 and Envisat respectively. Jason-1 was found to require no correction, performing well throughout the range of wave heights, although it is notably noisier than Envisat. An overall RMS difference between Jason-1 and buoy data of $0.227 \mathrm{~m}$ is found. Envisat has a tendency to overestimate low $H_{s}$ and underestimate high $H_{s}$. A linear correction reduces the RMS difference by $7 \%$, from $0.219 \mathrm{~m}$ to $0.203 \mathrm{~m}$.

Though peripheral to the overall aims of this thesis, a significant finding of this work was the identification of a systematic difference in the $H_{s}$ being reported by MEDS and NDBC buoy networks. Using the altimeter data as a common reference, it is estimated that MEDS buoys are underestimating $H_{s}$ relative to NDBC buoys by about $10 \%$. Investigations as to the cause of this discrepancy are ongoing.

\section{Wind Verifications}

Chapter 3 presented the first direct assessment of the ACCESS marine surface winds. Comparisons against QuikSCAT scatterometer data identified a low bias, with $U_{10}$ underestimated by approximately $8 \%$. Significant regional variation is also apparent. Low biases are evident in the lee of islands, and along coastlines, most prominently on the mid-latitude east coasts of the Southern Ocean continents. Poor performance is noted at high wind speeds, with a lack of adequate resolution resulting in an inability to capture these extremes.

\section{Initial Wave Verifications}

Chapter 4 documented the set up and verification of WW3 under ACCESS forcing. Evaluation of a number of hindcasts was conducted, examining the impact of choices of source terms and numerics. A negative $H_{s}$ bias is present in all hindcasts. This is attributed mainly to the negative bias in the ACCESS forcing winds. The identification of the forcing winds as the primary cause of bias seen in the wave model in this case, effectively answers one of the major questions of this thesis.

In this context, TC96 was found to over-predict in the long fetches of the Southern Ocean, resulting in over-predicted $H_{s}$ values on the Australian west coast. BAJ terms were found to produce a more consistent negative bias over 
the globe, with the exception of the eastern Tropical Pacific, due to a lack swell attenuation within these terms.

\section{Statistical Wind Corrections}

In Chapter 5, a number of techniques for statistically correcting the wind speed have been explored, based on comparisons against QuikSCAT observations. The simplest of these examined the application of a fixed correction over the entire global domain. While such corrections produced overall improvements, they were unable to adequately account for regional variation in $U_{10}$ bias over the globe. These regional biases were effectively removed with a spatially varying correction, calculated independently for each model grid point, retrospectively for the entire period.

These spatially varying corrections were then extended to vary in time. A method was proposed whereby automatically evolving corrections are calculated in real time from a moving window of historical comparisons between observations and preceding forecasts. A number of spatial and temporal learning windows were explored, with a 30 day learning window, calculated individually for each model grid point was found to give the best improvement in terms of model RMSE. This relatively long learning period effectively targeted the removal of synoptic scale systematic biases, while applying independent corrections at each model grid point enabled the removal of persistent biases on fine spatial scales, such as those present along coastlines and in the lee of islands. This technique addresses the need for geographically varying corrections, as well as eliminating the need to monitor and manually adjust these corrections with time. Correcting the winds in this way, has resulted in a reduction in $U_{10}$ RMSE of about $8 \%$ and regional biases in the winds have been effectively removed.

\section{The Effect of Correction on the Wave Field}

In Chapter 6, the spatial biases in the wave field resulting from three different wind fields with known spatial biases have been compared. These consist of the uncorrected ACCESS winds, and those that have been corrected with both the static homogeneous corrections, and the spatially and temporally varying learned corrections determined in Chapter 5. With respect to the overall questions of this thesis, the follow conclusions are drawn for wave model runs performed using BAJ 
source terms:

- To what extent can the large scale systematic biases in the waves be attributed to the forcing winds?

- For wave model runs forced with uncorrected ACCESS winds, the majority of the negative bias can be attributed to a negative bias in the forcing. This result emphasizes the importance of analysing the two in parallel.

- The reduction of systematic wind biases does not result in universal improvement in modelled $H_{s}$. In some situations, internal wave model error is compensated by error in the forcing winds.

- In a general sense, it is clear that spatial bias in the wave fields is highly sensitive to that of the forcing wind fields. This applies not only to the overall bias, but its spatial variation.

- Do statistical adjustments to remove these winds biases lead to better wave forecasts?

- The improvement in the wind bias as a result of corrections is not matched in the modelled $H_{s}$ results overall.

- Large regional variation is, however, apparent. Results in the Northern Hemisphere indicate strong improvements (approximately 17\% in terms of RMSE), while in general, results have been degraded in the Southern Hemisphere. The Tropics too show degradation, due primarily to excess swell on the eastern boundaries of the major ocean basins.

- Small scale features such as coastal biases show improvement

- What are the residual wave biases?

- A positive bias is present in the Southern Hemisphere due primarily to an overestimation of high $H_{s}$ values in the Southern Ocean storm tracks.

- A positive bias is present in the east Pacific and east Indian Ocean. This is due both to the over-prediction of waves in the Southern Ocean and the inherent lack of swell attenuation in the BAJ source terms. 
- Smaller scale features are apparent, such as a positive bias off the Cape of Good Hope, and a negative bias off Cape Horn. The origin of these features is unclear. Current effects that are not included in the wave model are a possible explanation. The effect of currents on the wind correction methods applied may also be a factor.

For TC96 source terms, conclusions are generally similar. TC96 terms appear to respond well to spatially varying wind corrections in the Northern Hemisphere, similar to the BAJ results. On the large scale, spatial bias is greatly reduced when forced with spatially unbiased winds, and RMSE is reduced by almost 20\%. Results are degraded in the Southern Hemisphere, even more than the BAJ terms. Where low and moderate waves appear well captured in the case of BAJ, they are overestimated by TC96 terms, while TC96 produces better values at high wave heights.

The sensitivity of the wave model to the forcing winds requires some degree of tuning to be undertaken in order to achieve unbiased wave fields under a given wind forcing. Some tuning of the BAJ wind input growth term $\beta_{\max }$ was found to mitigate much of the $H_{s}$ bias when forced with ACCESS winds. However, it was shown that such tuning can not account for the spatial structure of the wind bias. Better spatial bias could be achieved by tuning the wind input to winds that had been corrected using the spatially and temporally varying wind corrections.

Overall, it is clear that by performing the spatially and temporally varying wind corrections applied in this work, the contribution of the systematic wind error to the $H_{s}$ bias is significantly reduced, allowing inherent wave model error to be more effectively isolated.

\subsection{Further Work}

The extensive coverage of the QuikSCAT scatterometer data has been shown to provide ample data density for the purposes of constructing an observationally based learned gridded correction. However, the simple corrections applied here do not adequately account for the apparent significant underestimations at high wind speeds. The non-linear dependence of wave growth on wind speed means that mean wave biases are disproportionately sensitive to wind biases at these high winds. This has has been well demonstrated in several recent studies (e.g. Chawla 
et al., 2009, 2011). The application of higher order corrections or percentile based corrections (e.g. Hemer et al., 2011) would be worth investigating as a means of improving high wind speeds above and beyond what is achievable with the simple corrections applied here.

This work has only examined a single, short forecast period, focusing mainly in the removal of systematic bias. The strength of this approach, is that it can be independently applied across the range of available forecast periods. In fact, as inherent model errors can be expected to become more prevalent with increasing forecast period, such bias correction techniques have been shown to give greater relative gains at these longer forecast periods (e.g. Durrant et al., 2009b). Given that operational weather centres are usually receiving a number of surface wind products from other centres in real time, the possibility also exists to extend this work to include the weighted compositing aspects of a true OCF scheme. If the gains seen by Durrant et al. (2009b) at site locations could be replicated over the entire domain, significant improvements in forecast skill could be expected in addition to the reduction in bias shown here.

A number of deficiencies in the wave model have been highlighted when forced with corrected winds. Under-attenuation of swell in the BAJ terms is a known problem, and is addressed in the work of Ardhuin et al. (2010). The problem of overestimation of high waves by the BAJ terms in the Southern Ocean warrants further attention, with the possibility of a tendency for residual growth above PM saturation limits being a point of concern. This is likely due in part to an overestimation of the drag coefficient at these high wind speeds. TC96 terms have likewise been found to over-predict $H_{s}$, primarily in the low to moderate range, with high wave heights captured more accurately than the BAJ terms, likely due to a capping of the drag coefficient. No exploration for this tendency to overpredict has been carried out here, nor is the differing nature of the over-prediction between BAJ and TC96 terms examined in detail. A simple comparison of fetch and duration limited growth for these terms, such as that carried out by Rogers (2002) in the case of TC96 and WAM4 terms, would be a valuable exercise. An examination of the residual growth behaviour of these terms, similar to the long fetch experiments carried out by Bender (1996), for example, for the WAM4 terms would also be beneficial.

It is worth noting here however, the current work occurring under the NOPP project, a NOAA / U.S. Navy / United States Army Corps of Engineers (USACE) 
collaboration funded over four years beginning at the start of the 2010 financial year (Tolman et al., 2011). This project has the broad aims of, among other things, developing new spectral source terms within WW3 incorporating recent advances in theoretical knowledge of wind input and in particular, wave breaking and dissipation. In this context, the source terms of BAJ and TC96 are unlikely to receive significant additional attention.

The work presented here has focused on large scale, time averaged, systematic bias, as determined by comparison with altimeter and scatterometer data for the modelled $H_{s}$ and forcing winds respectively. Both qualitative and quantitative claims made here could be strengthened through the consideration of statistical uncertainty and confidence limits on these results. The application of field significance (e.g. Livezey and Chen, 1983; Elmore et al., 2006) for example, would be a valuable means of assessing the significance of the spatial biases described.

Further analysis of time series data from buoys would also be beneficial. The limited buoy data used here, from the Australian network, does not include wind observations. Independent verification of the applied wind correction methods using buoy data from international networks would valuable further assessment. Similarly, the ability to monitor wave time series would bring further insight to the wave hindcast results.

The signature of ocean surface current effects can be seen in both the scatterometer based wind verifications, as well as in the modelled wave results presented in this work. In hindsite, the neglect of currents adds some uncertainty to the results, both in terms of the proposed correction methodology and their contribution to wave biases on global scales. The increasing skill of operational ocean modelling and the availability of modelled ocean current forecasts in real time (e.g. Brassington et al., 2007) provides the opportunity for them to be explicitly accounted for. Further examination of the effect of ocean currents on the wind methodology, as well as the influence of currents on global scales (i.e. extending the work of Hersbach and Bidlot (2008), is warranted.

Finally, this study has been performed on a limited period, covering the Southern Hemisphere winter months. Conclusions drawn have greatly differed for the Northern and Southern Hemispheres. In general, it is unclear whether these differences are specific to these respective hemispheres, or whether they are simply a result of seasonal differences. This could be addressed by performing a similar analysis for the Northern Hemisphere winter months. For reasons previously 
discussed, i.e. unavailability of winds for this period, this is left for further work.

\subsection{Closing Remarks}

Ultimately, the question of whether such corrections to the forcing winds can be justified in an operational environment must be asked. With respect to the production of accurate wave forecasts, without applying any tuning to the wave model, in the Northern Hemisphere, clear gains have been made. In the Southern Hemisphere, and specifically the Australian region, such corrections alone are probably unjustified in an operational environment, with both source term packages examined here producing strong overestimations of $H_{s}$ in this region when forced with corrected winds. The source terms used in modern operational wave models, have reasonably been tuned to produce accurate wave forecasts under the winds primarily used during their development. As such, in most cases, tuning of these terms will be required to produce accurate forecasts under arbitrary winds. To an extent, this tuning can compensate for wind errors.

Arguments still exist for the application of a learned wind corrections scheme however. Evolving error characteristics in the forcing winds is a reality of any operational wave forecasting system. By tuning the model to these corrected winds, the problem of maintaining the tuned state of the model is reduced, as the automatically evolving wind corrections make this retuning somewhat redundant. The ability to account for spatial variation in the systematic wind error is also something that is not easily accounted for by simply wave model tuning alone, nor can such tuning account for variation in wind speed bias with forecast period.

It is clear from this work is that a knowledge of the error characteristics of the forcing wind is an important aspect of any evaluation of a global wave modelling system. In the specific case of the WW3 model forced with ACCESS winds examined here, it is clear that the negative $H_{s}$ bias has its origins primarily in the forcing. By performing statistical corrections on the wind prior to forcing the wave model, systematic error in the $H_{s}$ has been greatly reduced in some areas; it has been worsened in others. In the context of wave model development, it is emphasised here that by applying these corrections, the contribution of the systematic wind error to that of the waves is reduced, allowing inherent wave model error to be more effectively isolated and addressed. 


\section{References}

Abdalla, S., Global Validation of ENVISAT Wind, Wave and Water Vapour Products from RA-2, MWR, ASA and MERIS, ESA Contract Report 17585, 2006.

Abdalla, S., and J.-R. Bidlot, Wind gustiness and air density effects and other key changes to wave model in CY25R1, ECMWF Technical Memorandum, (273), 2002 .

Abdalla, S., J.-R. Bidlot, and P. A. E. M. Janssen, Jason Altimeter Wave Height Verification and Assimilation, in Proceedings of the Seventh International Conference on the Mediterranean Coastal Environment (MEDCOAST 05), Kusadasi, Turkey, 25-29 Oct. 2005, pp. 1179-1185, 2005.

Alves, J.-H. G. M., Numerical modeling of ocean swell contributions to the global wind-wave climate, Ocean Modelling, 11, 98-122, 2006.

Alves, J.-H. G. M., M. L. Banner, and I. R. Young, Revisiting the PiersonMoskowitz Asymptotic Limits for Fully Developed Wind Waves, Journal of Physical Oceanography, 33(7), 1301-1323, doi:10.1175/1520-0485(2003) 033〈1301:RTPALF $\rangle$ 2.0.CO;2, 2003.

Ardhuin, F., L. Bertotti, J.-R. Bidlot, L. Cavaleri, V. Filipetto, J.-M. Lefevre, and P. Wittmann, Comparison of wind and wave measurements and models in the Western Mediterranean Sea, Ocean Engineering, 34(3-4), 526-541, doi: 10.1016/j.oceaneng.2006.02.008, 2007.

Ardhuin, F., F. Collard, B. Chapron, P. Queffeulou, J. Filipot, and M. Hamon, Spectral wave dissipation based on observations: a global validation, in Proceedings of Chinese-German Joint Symposium on Hydraulics and Ocean Engineering, Darmstadt, Germany, pp. 391-400, 2008. 
Ardhuin, F., B. Chapron, and F. Collard, Observation of swell dissipation across oceans, Geophysical Research Letters, 36(6), 1-6, doi:10.1029/2008GL037030, 2009 .

Ardhuin, F., W. E. Rogers, A. V. Babanin, J.-F. Filipot, R. Magne, A. Roland, A. van der Westhuysen, P. Queffeulou, B. Lee, L. Aouf, and F. Collard, Semiempirical Dissipation Source Functions for Ocean Waves. Part I: Definition, Calibration, and Validation, Journal of Physical Oceanography, 40(9), 1917-1941, doi:10.1175/2010JPO4324.1, 2010.

Ardhuin, F., J. Tournadre, P. Queffeulou, F. Girard-Ardhuin, and F. Collard, Observation and parameterization of small icebergs: drifting breakwaters in the Southern Ocean, Ocean Modelling, 1, 2-7, doi:10.1016/j.ocemod.2011.03.004, 2011 .

Atlas, R., R. Hoffman, S. Leidner, J. Sienkiewicz, T. Yu, S. Bloom, E. Brin, J. Ardizzone, J. Terry, and D. Bungato, The effects of marine winds from scatterometer data on weather analysis and forecasting, Bulletin of the American Meteorological Society, 82(9), 1965-1990, doi:10.1175/1520-0477(2001) 082〈1965:TEOMWF $>2.3 . \mathrm{CO} ; 2,2001$.

Bender, L. C., Modification of the physics and numerics in a third-generation ocean wave model, Journal of Atmospheric and Oceanic Technology, 13, 726$750,1996$.

Bender, L. C., and T. J. Glowacki, The assimilation of altimeter data into the Australian wave model, Aust. Met. Mag, 45, 41-48, 1996.

Bertotti, L., and L. Cavaleri, Accuracy of the modelled wind and wave fields in enclosed seas, Tellus, 56A(2), 167-175, 2004.

Bidlot, J.-R., A Modified Set Up of the Advection Scheme in the ECMWF Wave Model, ECMWF Technical Memorandum, 237, 1997.

Bidlot, J.-R., D. J. Holmes, P. A. Wittmann, R. Lalbeharry, and H. S. Chen, Intercomparison of the performance of operational ocean wave forecasting systems with buoy data, Weather and Forecasting, 17, 287-310, 2002.

Bidlot, J.-R., P. A. E. M. Janssen, and S. Abdalla, A revised formulation of ocean wave dissipation and its model impact., ECMWF Technical Memorandum, 509, 2007. 
Bidlot, J.-R., T. H. Durrant, and P. Queffeulou, Assessment of the systematic differences in wave observations from moorings, in JCOMM Technical Workshop on Wave Measurements from Buoys, 2 - 3 October 2008, New York, U.S., 2008 .

Booij, N., and L. H. Holthuijsen, Propagation of ocean waves in discrete spectral wave models, Journal of Computational Physics, 68(2), 307-326, 1987.

Booij, N., R. C. Ris, and L. H. Holthuijsen, A third-generation wave model for coastal regions, Part I, Model description and validation, Journal of Geophysical Research, 104, 7649-7666, 1999.

Brassington, G., T. Pugh, C. Spillman, E. W. Schulz, H. Beggs, A. Schiller, and P. R. Oke, BLUElink\&gt; Development of operational oceanography and servicing in Australia, Journal of Research and Practice in Information Technology, 39(2), 151-164, 2007.

Caires, S., and A. Sterl, Validation of ocean wind and wave data using triple collocation, Journal of Geophysical Research, 108(C3), 3098, doi:10.1029/ 2002JC001491, 2003.

Caires, S., A. Sterl, J.-R. Bidlot, N. Graham, and V. Swail, Intercomparison of different wind-wave reanalyses, Journal of Climate, 17, 1893-1913, 2004.

Carayon, G., N. Steunou, J. L. Courri `ere, and P. Thibaut, Poseidon-2 Radar Altimeter Design and Results of In-Flight Performances, Marine Geodesy, 26, 159-165, 2003.

Cardone, V. J., H. Graber, P. Jensen, S. Hasselmann, and M. Caruso, In search of the true surface wind field in SWADE IOP-1: Ocean wave modelling perspective, Global Atmos. Ocean Syst., 3, 107-150, 1995.

Cardone, V. J., R. E. Jensen, D. T. Resio, V. R. Swail, and A. T. Cox, Evaluation of contemporary ocean wave models in rare extreme events: The "Halloween storm" of October 1991 and the "storm of the century" of March 1993, Journal of Atmospheric and Oceanic Technology, 13, 198-230, 1996.

Cardone, V. J., A. T. Cox, E. L. Harris, E. A. Orelup, M. J. Parsons, and H. C. Graber, Impact of QuikSCAT Surface Marine Winds on Wave Hindcasting, in 12th International Workshop on Wae Hindcasting and Forecasting Oahu, Hawaii November 14-19, 2004. 
Cavaleri, L., Wave models and input wind, in Wave Dynamics and Modelling of Ocean Waves, edited by G. J. Komen, S. Hasselmann, M. Donelan, L. Cavaleri, and P. Janssen, pp. 283-284., Cambridge University Press, 1994.

Cavaleri, L., Wave Modeling - Missing the Peaks, Journal of Physical Oceanography, 39(11), 2757, doi:10.1175/2009JPO4067.1, 2009.

Cavaleri, L., and L. Bertotti, The improvement of modelled wind and wave fields with increasing resolution, Ocean Engineering, 33(5-6), 553-565, doi:10.1016/ j.oceaneng.2005.07.004, 2006.

Chalikov, D. V., The parameterization of the wave boundary layer, Journal of Physical Oceanography, 25(6), 1333-1349, 1995.

Chalikov, D. V., and M. Belevich, One-dimensional theory of the wave boundary layer, Boundary-Layer Meteorology, 63(1), 65-96, 1993.

Challenor, P. G., and P. D. Cotton, The joint calibration of altimeter and in situ wave heights, in Advances in the Applications of Marine Climatology - The Dynamic Part of the WMO Guide to the Applications of Marine Climatology, WMO/TD-No. 1081, WMO Geneva., 2001.

Chawla, A., and H. L. Tolman, Automated grid generation for WAVEWATCH III, Tech. Note 254, NOAA/NWS/NCEP/MMAB, 2007.

Chawla, A., and H. L. Tolman, Obstruction Grids for Spectral Wave Models, Ocean Modelling, 22, 12-25, doi:10.1016/j.ocemod.2008.01.003, 2008.

Chawla, A., H. L. Tolman, J. L. Hanson, E.-m. Devaliere, and V. M. Gerald, Validation of a Multi-Grid WAVEWATCH III Modeling System, in 11th International Workshop on Wae Hindcasting and Forecasting \& Coastal Hazards Symposium, Halifax, Canada October 18-23, 281, 2009.

Chawla, A., S. Deanna, and H. L. Tolman, A Thirty Year Wave Hindcast Using The Latest NCEP Climate Forecast System Reanalysis Winds, in 12th International Workshop on Wae Hindcasting and Forecasting $\&$ Coastal Hazards Symposium, Kohala Coast, Hawaii, October 30-November 4, 2011, 2011.

Chelton, D. B., and M. H. Freilich, Scatterometer-based assessment of 10-m wind analyses from the Operational ECMWF and NCEP Numerical Weather Prediction Models, Monthly Weather Review, 133, 409-429, 2005. 
Chelton, D. B., M. G. Schlax, M. H. Freilich, and R. F. Milliff, Satellite measurements reveal persistent small-scale features in ocean winds., Science, 303(5660), 978-83, doi:10.1126/science.1091901, 2004.

Chelton, D. B., M. H. Freilich, J. Sienkiewicz, and J. Von Ahn, On the use of QuikSCAT scatterometer measurements of surface winds for marine weather prediction, Monthly Weather Review, 134, 2055-2071, 2006.

Collard, F., F. Ardhuin, and B. Chapron, Persistency of ocean swell fields observed from space, Journal of Geophysical Research, (1), 1-11, 2008.

Cotton, P. D., and D. J. T. Carter, Cross calibration of TOPEX, ERS-1, and GEOSAT wave heights, Journal of Geophysical Research, 99, 25,025-25,033, 1994.

Cotton, P. D., D. J. T. Carter, P. G. Challenor, and V. Y. Kareev, A coordinated programme for global calibration/validation of altimeter sea state data, AVISO Newsletter, 8, 41-42, 2001.

Cotton, P. D., P. G. Challenor, and J.-M. Lefevre, Calibration of ENVISAT and ERS-2 wind and wave data through comparison with in-situ data and wave emodel analysis fields, 2004.

Desai, S. D., and P. Vincent, Statistical Evaluation of the Jason-1 Operational Sensor Data Record, Marine Geodesy, 26, 187-199, 2003.

Donelan, M. A., On the limiting aerodynamic roughness of the ocean in very strong winds, Geophysical Research Letters, 31(18), 1-5, doi:10.1029/ 2004GL019460, 2004.

Durrant, T. H., and D. J. M. Greenslade, Spatial Evaluations of ACCESS Marine Surface Winds using Scatterometer Data, Australian Meteorological and Oceanographic Journal - Submittted.

Durrant, T. H., and D. J. M. Greenslade, Evaluation and Implementation of AUSWAVE, CAWCR Technical Report, 41, 2011.

Durrant, T. H., D. J. M. Greenslade, and I. Simmonds, Validation of Jason-1 and Envisat Remotely Sensed Wave Heights, Journal of Atmospheric and Oceanic Technology, 26(1), 123, doi:10.1175/2008JTECHO598.1, 2009a. 
Durrant, T. H., D. J. M. Greenslade, and F. Woodcock, Consensus Forecasts of Modeled Wave Parameters, Weather and Forecasting, 24(2), 492, doi:10.1175/ 2008WAF2222143.1, 2009b.

Elmore, K. L., M. E. Baldwin, and D. M. Schultz, Field Significance Revisited: Spatial Bias Errors in Forecasts as Applied to the Eta Model, Monthly Weather Review, 134(2), 519-531, doi:10.1175/MWR3077.1, 2006.

EUMETSAT, ASCAT Product Guide - http://oiswww.eumetsat.org/WEBOPS/epspg/ASCAT/ASCAT-PG-index.htm, Tech. rep., 2010.

Faugere, Y., J. Dorandeu, F. Lefevre, N. Picot, and P. Femenias, Envisat ocean altimetry performance assessment and cross-calibration, Sensors, 6, 100-130, 2006 .

Feng, H., D. Vandemark, B. Chapron, Y. Quilfen, and B. Beckley, Assessment of wind-forcing impact on a global wind-wave model using the TOPEX altimeter, Ocean Engineering, 33, 1431-1461, 2006.

Freilich, M. H., Validation of vector magnitude datasets: Effects of random component errors, Journal of Atmospheric and Oceanic, pp. 695-703, 1997.

Freilich, M. H., and B. A. Vanhoff, The accuracy of preliminary WindSat vector wind measureinrients: Comparisons with NDBC buoys and QuikSCAT, IEEE Transactions on Geoscience and Remote Sensing, 44, 622-637, 2006.

Golding, B., A wave prediction system for real-time sea state forecasting, Quarterly Journal of the Royal Meteorological Society, 109(460), 393-416, 1983.

Gommenginger, C. P., M. A. Srokosz, P. G. Challenor, and P. D. Cotton, Measuring ocean wave period with satellite altimeters: A simple empirical model, Geophys. Res. Lett, 30, 2150, 2003.

Greenslade, D. J. M., Upgrades to the Bureau of Meteorology's Ocean Wave Forecasting System, BMRC Research Report, 79, 2000.

Greenslade, D. J. M., and I. R. Young, A validation of ERS-2 Fast Delivery Significant Wave Height, BMRC Research Report, 97, 2004a.

Greenslade, D. J. M., and I. R. Young, Background errors in a global wave model determined from altimeter data, Journal of Geophysical Research, 109(C9), C09,007, doi:10.1029/2004JC002324, 2004b. 
Greenslade, D. J. M., E. W. Schulz, J. D. Kepert, and G. R. Warren, The impact of the assimilation of scatterometer winds on surface wind and wave forecasts, Journal of Atmospheric and Ocean Science, 10, 261-287, 2005.

Grumbine, R. W., Sea Ice Prediction Environment: Documentation, Tech. Note 121, NOAA/NWS/NCEP/MMAB, 121, 1996.

Hardy, T., A wave model for the Great Barrier Reef, Ocean Engineering, 28(1), 45-70, doi:10.1016/S0029-8018(99)00057-8, 2001.

Hasselmann, K., On the spectral dissipation of ocean waves due to white capping, Boundary-Layer Meteorology, 6(1), 107-127, 1974.

Hasselmann, K., T. Barnett, E. Bouws, H. Carlson, D. Cartwright, K. Enke, J. Ewing, H. Gienapp, D. Hasselmann, P. Kruseman, and Others, Measurements of wind-wave growth and swell decay during the Joint North Sea Wave Project (JONSWAP), Ergnzungsheft zur Deutschen Hydrographischen Zeitschrift Reihe, 8(12), 1973.

Hasselmann, S., K. Hasselmann, J. Allender, and T. Barnett, Computation and parameterizations of the nonlinear energy transfer in a gravity-wave spectrum. Part II: Parameterizations of the nonlinear energy transfer for application in wave models, J. Phys. Oceanogr., 15, 1378-1391, 1985.

Hemer, M. A., K. L. McInnes, and R. Ranasinghe, Climate and variability bias adjustment of climate model-derived winds for a southeast Australian dynamical wave model, Ocean Dynamics, (2007), doi:10.1007/s10236-011-0486-4, 2011.

Hersbach, H., and J.-R. Bidlot, The relevance of ocean surface current in the ECMWF analysis and forecast system, in Workshop on Ocean-Atmosphere Interactions, pp. 61-73, 2008.

Holt, M., Improvement to UKMO wave model swell dissipation and performance in light winds, Met Office Forecasting Research Division Tech. Rep, 119, 12, 1994.

Holthuijsen, L. H., N. Booij, R. Ris, I. G. Haagsma, A. Kieftenburg, and E. E. Kriezi, SWAN Cycle III version 40.11 user manual, Delft University of Technology, The Netherlands, 2002. 
Huang, N. E., S. R. Long, and L. F. Bliven, On the Importance of the Significant Slope in Empirical Wind-Wave Studies, Journal of Physical Oceanography, 11, 569-573, 1981.

Hubert, W. E., A preliminary report on numerical sea condition forecasts, Monthly Weather Review, 85, 200-204, 1957.

Hwang, P. A., W. J. Teague, G. A. Jacobs, and D. W. Wang, A statistical comparison of wind speed, wave height, and wave period derived from satellite altimeters and ocean buoys in the Gulf of Mexico region, Journal of Geophysical Research, 103, 10,451-10,468, 1998.

Isaksen, L., and P. A. E. M. Janssen, Impact of ERS scatterometer winds in ECMWF's assimilation system, Quarterly Journal of the Royal Meteorological Society, 130(600), 1793-1814, doi:10.1256/qj.03.110, 2004.

Isobe, T., E. D. Feigelson, M. G. Akritas, and G. J. Babu, Linear regression in astronomy., Astrophysical Journal, 364, 104-113, 1990.

Janssen, P. A. E. M., Quasi-linear Theory of Wind-Wave Generation Applied to Wave Forecasting, Journal of Physical Oceanography, 21, 1631-1642, 1991.

Janssen, P. A. E. M., ECMWF wave modeling and satellite altimeter wave data, in Satellites, Oceanography and Society, vol. 3, edited by D. Halpern, pp. 26392640, 2000.

Janssen, P. A. E. M., Progress in ocean wave forecasting, Tech. rep., ECMWF, 2007.

Janssen, P. A. E. M., Progress in ocean wave forecasting, Journal of Computational Physics, 227(7), 3572-3594, doi:10.1016/j.jcp.2007.04.029, 2008.

Janssen, P. A. E. M., K. Hasselmann, S. Hasselmann, and G. Komen, Parameterization of source terms and the energy balance in a growing wind sea, in Dynamics and Modelling of Ocean Waves, Cambridge University Press, 1994.

Janssen, P. A. E. M., B. Hansen, and J.-R. Bidlot, Verification of the ECMWF wave forecasting system against buoy and altimeter data, Weather and Forecasting, 12, 763-784, 1997. 
Kara, A. B., A. J. Wallcraft, C. N. Barron, H. E. Hurlburt, and M. A. Bourassa, Accuracy of $10 \mathrm{~m}$ winds from satellites and NWP products near land-sea boundaries, Journal of Geophysical Research, 113(C10), C10,020, doi: 10.1029/2007JC004516, 2008.

Kelly, K., S. Dickinson, M. McPhaden, and G. Johnson, Ocean currents evident in satellite wind data, Geophysical Research Letters, 28(12), 2469-2472, 2001.

Kelly, K. A., Wind data: A promise in peril, Science, 303, 962-963, 2004.

Kepert, J. D., D. J. M. Greenslade, and G. D. Hess, Assessing and Improving the Marine Surface Winds in the Bureau of Meteorology Numerical Weather Prediction Systems, BMRC Research Report, (105), 2005.

Khandekar, M. L., and R. Lalbeharry, An Evaluation of Environment Canada's Operational Ocean Wave Model Based on Moored Buoy Data, Weather and Forecasting, 11 (2), 137-152, doi:10.1175/1520-0434(1996)011〈0137:AEOECO〉 2.0.CO;2, 1996 .

Komen, G., S. Hasselmann, and K. Hasselmann, On the existence of a fully developed wind-sea spectrum, J. Phys. Oceanogr, 14(8), 1271-1285, 1984.

Komen, G., L. Cavaleri, M. A. Donelan, and S. Hasselmann, Dynamics and modelling of ocean waves, Cambridge University Press, 1996.

Leonard, B., A stable and accurate convective modelling procedure based on quadratic upstream interpolation, Computer methods in applied mechanics and engineering, 19(1), 59-98, 1979.

Leonard, B., The ULTIMATE conservative difference scheme applied to unsteady one-dimensional advection, Computer methods in applied mechanics and engineering, 88(1), 17-74, 1991.

Lionello, P., H. Gunther, and P. A. E. M. Janssen, Assimilation of altimeter data in a global third-generation wave model, Journal of Geophysical Research, 97(C9), 14,453, 1992.

Livezey, R., and W. Chen, Statistical field significance and its determination by Monte Carlo techniques(in meteorology), Monthly Weather Review, 111, 46-59, 1983. 
Longuet-Higgins, M. S., On the statistical distributions of sea waves, J. mar. Res., $11(3), 245-265,1952$.

Lorenc, A., Analysis methods for numerical weather prediction, Quarterly Journal of the Royal Meteorological Society, 112(474), 1177-1194, 1986.

Lungu, T., and P. Callahan, QuikSCAT science data product users manual: Overview and geophysical data products, Version D-18053-Rev A, 3, 91, 2006.

Mackay, E. B. L., P. G. Challenor, C. H. Retzler, and C. P. Gommenginger, A parametric model for ocean wave period from $\mathrm{Ku}$ band altimeter data, Journal of Geophysical Research, 113(C3), 1-16, doi:10.1029/2007JC004438, 2008.

Mears, C., F. Wentz, and D. Smith, SeaWinds on QuikSCAT normalized objective function rain flag, Product description version, 1, 2000.

Menard, Y., L. L. Fu, P. Escudier, F. Parisot, J. Perbos, P. Vincent, S. Desai, B. Haines, and G. Kunstmann, The Jason-1 Mission, Marine Geodesy, 26, 131-146, 2003.

Miles, J., On the generation of surface waves by shear flows, Journal of Fluid Mechanics, 3, 185-204, 1957.

Monahan, A. H., The Probability Distribution of Sea Surface Wind Speeds. Part I: Theory and SeaWinds Observations, Journal of Climate, 19(4), 497, doi: 10.1175/JCLI3640.1, 2006.

Monaldo, F., Expected differences between buoy and radar altimeter estimates of wind-speed and significant wave height and their implications on buoy altimeter comparisons, Journal of Geophysical Research, 93, 2285-2302, 1988.

Naderi, F. M., M. H. Freilich, and D. G. Long, Spaceborne radar measurement of wind velocity over the ocean-an overview of the NSCAT scatterometer system, Proceedings of the IEEE, 79(6), 850-866, 1991.

NGDC, 2-minute gridded global relief data (ETOPO2), 2006.

NMOC, Analysis and Prediction Operations Bulletin No . 74 Operational Implementation of the Gridded OCF System Overview of the Gridded OCF system, Operations Bulletin, (74), 1-35, 2008. 
NMOC, Operational implementation of the ACCESS Numerical Weather Prediction systems, Operations Bulletin, (83), 1-35, 2010a.

NMOC, Operational Implementation of the new Gridded OCF and PME suite, Operations Bulletin, (82), 1-13, 2010b.

NRL, Digital bathymetry data base 2-minute resolution v. 3 (DBB2)., 2006.

Perrie, W., W. Zhang, M. A. Bourassa, H. Shen, and P. W. Vachon, Impact of Satellite Winds on Marine Wind Simulations, Weather and Forecasting, 23(2), 290, doi:10.1175/2007WAF2006093.1, 2008.

Petkovic, P., and C. Buchanan, Australian Bathymetry and Topography Grid [Digital Dataset], Geoscience Australia, Canberra, 2002.

Pierson, W., and L. Moskowitz, A proposed spectral form for fully developed wind seas based on the similarity theory of SA Kitaigorodskii, Journal of Geophysical Research, 69(24), 5181-5190, 1964.

Powell, M. D., P. J. Vickery, and T. A. Reinhold, Reduced drag coefficient for high wind speeds in tropical cyclones, Nature, 422(March), 279, 2003.

Puri, K., G. S. Dietachmayer, G. A. Mills, N. E. Davidson, R. A. Bowen, and L. W. Logan, The new BMRC Limited Area Prediction System, LAPS, Aust. Meteor. Mag, 47, 203-223, 1998.

Queffeulou, P., Cross validation of ENVISAT RA-2 significant wave height, sigma0 and wind speed, CCVT final report, May, 2003.

Queffeulou, P., Long-Term Validation of Wave Height Measurements from Altimeters, Marine Geodesy, 27, 495-510, 2004.

Queffeulou, P., Altimeter wave height validation: an update, in Ocean Surface Topography Science Team 2006 meeting, Venice, 16-18 March, 2006.

Quilfen, Y., B. Chapron, F. Collard, and M. Serre, Calibration/Validation of an Altimeter Wave Period Model and Application to TOPEX/Poseidon and Jason-1 Altimeters, Marine Geodesy, 27, 535-549, 2004.

Rawlins, F., S. Ballard, K. Bovis, A. Clayton, D. Li, G. Inverarity, A. Lorenc, and T. Payne, The Met Office global four-dimensional variational data assimilation scheme, Quarterly Journal of the Royal Meteorological Society, 133(623), 347362, doi:10.1002/qj, 2007. 
Ray, R. D., and B. Beckley, Simultaneous Ocean Wave Measurements by the Jason and Topex Satellites, with Buoy and Model Comparisons, Marine Geodesy, 26, 367-382, 2003.

Resti, A., J. Benveniste, M. Roca, G. Levrini, and J. Johannessen, The Envisat Radar Altimeter System(RA-2), ESA Bulletin, 98, 94-101, 1999.

Rogers, W. E., The U.S. Navy's Global Wind-Wave Models: An Investigation into Sources of Errors in Low-Frequency Energy Predictions, Naval Research Lab Tech Report, (NRL/FR/7320 - 02-10,035), 2002.

Rogers, W. E., and P. Wittmann, Quantifying the Role of Wind Field Accuracy in the US Navy's Global Ocean Wave Nowcast/Forecast System, Naval Research Lab Tech Report, (NRL/MR/732-02-8290), 2002.

Rogers, W. E., P. A. Wittmann, D. W. C. Wang, R. M. Clancy, and Y. L. Hsu, Evaluations of global wave prediction at the fleet numerical meteorology and oceanography center, Weather and Forecasting, 20, 745-760, 2005.

RSS, Remote Sensing Systems - http://www.ssmi.com/qscat/qscat_description.html, 2010 .

Schulz, E. W., J. Kepert, and D. J. M. Greenslade, An Assessment of Marine Surface Winds from the Australian Bureau of Meteorology Numerical Weather Prediction Systems, Weather and Forecasting, 22, 613-636, 2007.

Seaman, R., W. Bourke, P. Steinle, T. Hart, G. Embery, M. Naughton, and L. Rikus, Evolution of the Bureau of Meteorology's Global Assimilation and Prediction system. Part 1: Analysis and initialisation, Aust. Meteor. Mag, 44, $1-18,1995$.

Sharma, N., and E. D'Sa, Assessment and Analysis of QuikSCAT Vector Wind Products for the Gulf of Mexico: A Long-Term and Hurricane Analysis, Sensors, 8(3), 1927-1949, doi:10.3390/s8031927, 2008.

Smith, W., and D. Sandwell, Global sea floor topography from satellite altimetry and ship depth soundings, Science, 27r7(5334), 1956, 1997.

Snyder, R. L., F. W. Dobson, J. A. Elliott, and R. B. Long, Array measurements of atmospheric pressure fluctuations above surface gravity waves, Journal of Fluid Mechanics Digital Archive, 102, 1-59, 1981. 
Soukissian, T., and C. Kechris, About applying linear structural method on ocean data: Adjustment of satellite wave data, Ocean Engineering, 34, 371-389, 2007.

Stoffelen, A., Toward the true near-surface wind speed: Error modeling and calibration using triple collocation, Journal of Geophysical Research, 103(C4), 7755-7766, 1998.

Stoffelen, A., and D. Anderson, Scatterometer Data Interpretation: Measurement Space and Inversion, Journal of Atmospheric and Oceanic Technology, 14(6), 1298, doi:10.1175/1520-0426(1997)014〈1298:SDIMSA〉2.0.CO;2, 1997.

Sun, F., and J.-Y. Yu, Impacts of Central America gap winds on the SST annual cycle in the eastern Pacific warm pool, Geophysical Research Letters, 33(6), 1-4, doi:10.1029/2005GL024700, 2006.

Sverdrup, H. U., and W. H. Munk, Wind, Sea and Swell: Theory of Relations for Forecasting, 1947.

Swail, V., R. Jensen, B. Lee, J. Turton, J. Thomas, S. Gulev, M. Yelland, P. Etala, D. Meldrum, W. Birkemeier, and Others, Wave measurements, needs and developments for the next decade, Proceedings of the" OceanObs, 9(1), 2010.

SWAMP Group, Ocean Wave Modelling, Plenum Press, 1985.

Taylor, K. E., Summarizing multiple aspects of model performance in a single diagram, J. Geophys. Res, 106(D7), 7183-7192, 2001.

Tolman, H. L., A Third-Generation Model for Wind Waves on Slowly Varying, Unsteady, and Inhomogeneous Depths and Currents, Journal of Physical Oceanography, 21(6), 782-797, doi:10.1175/1520-0485(1991)021<0782: ATGMFW $>2.0 . \mathrm{CO} ; 2,1991$.

Tolman, H. L., Effects of Numerics on the Physics in a Third-Generation WindWave Model, Journal of Physical Oceanography, 22, 1095-1111, 1992.

Tolman, H. L., On the selection of propagation schemes for a spectral wind-wave model, NWS/NCEP Office Note, 411(411), 30, 1995.

Tolman, H. L., Effects of observation errors in linear regression and bin-average analyses, Quarterly Journal of the Royal Meteorological Society, 124(547), 897917, doi:10.1002/qj.49712454712, 1998a. 
Tolman, H. L., Validation of NCEP's ocean winds for the use in wind wave models, The Global Atmosphere and Ocean System, 6(3), 243-268, 1998b.

Tolman, H. L., Validation of WAVEWATCH III version 1.15 for a global domain, Tech. Note 213, NOAA/NWS/NCEP/MMAB, 2002a.

Tolman, H. L., Alleviating the Garden Sprinkler Effect in wind wave models, Ocean Modelling, 4, 269-289, 2002b.

Tolman, H. L., Treatment of unresolved islands and ice in wind wave models, Ocean Modelling, 5(3), 219-231, doi:10.1016/S1463-5003(02)00040-9, 2003.

Tolman, H. L., User manual and system documentation of WAVEWATCH III TM version 3.14, Tech. Note 276, NOAA/NWS/NCEP/MMAB, 2009.

Tolman, H. L., and D. V. Chalikov, Source Terms in a Third-Generation Wind Wave Model, Journal of Physical Oceanography, 26, 2497-2518, 1996.

Tolman, H. L., B. Balasubramaniyan, L. D. Burroughs, D. V. Chalikov, Y. Y. Chao, H. S. Chen, and V. M. Gerald, Development and implementation of wind-generated ocean surface wave models at NCEP, Weather and Forecasting, 17, 311-333, 2002.

Tolman, H. L., M. Banner, and J. Kaihatu, The NOPP operational wave improvement project, in 12th International Workshop on Wae Hindcasting and Forecasting \& Coastal Hazards Symposium, Kohala Coast, Hawaii, October 30November 4, 2011, 294, 2011.

Tucker, M. J., and E. G. Pitt, Waves in ocean engineering, 2001.

WAMDIG:, S. Hasselmann, K. Hasselmann, P. A. E. M. Janssen, E. Bauer, G. J. Komen, L. Bertotti, P. Lionello, A. Guillaume, V. C. Cardone, J. A. Greenwood, and Others, The WAM model - A third generation ocean wave prediction model, J. Phys. Oceanogr, 18, 1775-1810, 1988.

Weller, R., M. A. Donelan, M. Briscoe, and N. Huang, Riding the Crest: A Tale of Two Wave Experiments., Bulletin of the American Meteorological Society, 72, 163-183, 1991.

Wessel, P., and W. H. F. Smith, A global, self-consistent, hierarchical, highresolution shoreline database, Journal of Geophysical Research, 101 (B4), 8741$8743,1996$. 
Wilks, D. S., Statistical methods in the atmospheric sciences, 1995.

WISE Group, L. Cavaleri, J.-H. G. M. Alves, F. Ardhuin, A. V. Babanin, M. L. Banner, K. Belibassakis, M. Benoit, M. A. Donelan, J. Groeneweg, T. Herbers, P. Hwang, P. A. E. M. Janssen, T. Janssen, I. Lavrenov, R. Magne, J. Monbaliu, M. Onorato, V. Polnikov, D. Resio, W. E. Rogers, A. Sheremet, J. McKee Smith, H. L. Tolman, G. van Vledder, J. Wolf, and I. Young, Wave modellingthe state of the art, Progress in Oceanography, 75(4), 603-674, doi:10.1016/j. pocean.2007.05.005, 2007.

Wittmann, P., and W. O'Reilly, WAM Validation of Pacific Swell, in 5th International Workshop on Wave Hindcasting and Forecasting, pp. 83-87, 1998.

Woodcock, F., and C. Engel, Operational consensus forecasts, Weather and forecasting, 20, 101-111, 2005.

Xie, S. P., W. T. Liu, Q. Liu, and M. Nonaka, Far-reaching effects of the Hawaiian Islands on the Pacific Ocean-atmosphere system., Science, 292(5524), 2057-60, doi:10.1126/science.1059781, 2001.

Xie, S.-P., H. Xu, W. S. Kessler, and M. Nonaka, AirSea Interaction over the Eastern Pacific Warm Pool: Gap Winds, Thermocline Dome, and Atmospheric Convection, Journal of Climate, 18(1), 5-20, doi:10.1175/JCLI-3249.1, 2005.

Yamartino, R. J., A Comparison of Several "Single-Pass" Estimators of the Standard Deviation of Wind Direction, Journal of Climate and Applied Meteorology, 23, 1362-1366, 1984.

Young, I., An experimental investigation of the role of atmospheric stability in wind wave growth, Coastal Engineering, 34(1-2), 23-33, doi:10.1016/ S0378-3839(98)00011-8, 1998.

Young, I. R., Seasonal variability of the global ocean wind and wave climate, International Journal of Climatology, 19, 931-950, 1999.

Young, I. R., and G. P. V. Vledder, A Review of the Central Role of Nonlinear Interactions in Wind-Wave Evolution, Philosophical Transactions: Physical Sciences and Engineering, 342, 505-524, 1993.

Yuan, X., High wind evaluation in the Southern Ocean, J. Geophys. Res, $109(845), 2004$. 


\section{Appendix A}

\section{LIST OF ACRONYMS}

4D-VAR Four-Dimensional Variational Assimilation

ACCESS Australian Community Climate Earth System Simulator (The operational atmospheric model run at the Bureau of Meteorology and analysed in this work)

ACC Antarctic Circumpolar Current

AUSWAM Australian WAve Model

ASCAT Advanced Scatterometer

Bureau Australian Bureau of Meteorology

CAWCR Centre for Australian Weather and Climate Research

CNES Centre National d'Etudes Spatiales

COAPS Center for Ocean-Atmospheric Prediction Studies

R Correlation Coefficient

DA Data Assimilation

ECMWF European Centre for Medium-Range Weather Forecasts

ENSO El Niño Southern Oscillation

ERS-1 European Remote Sensing Satellite - 1

ERS-2 European Remote Sensing Satellite - 2

ESA European Space Agency

FD Fast Delivery

FNMOC Fleet Numerical Meteorology and Oceanography Center 
GASP Global ASsimilation and Prediction System

GEOSAT GEOdesy SATellite

GEOS-3 Geodynamics Experimental Ocean Satellite

GFO Geosat Follow On

GFS Global Forecasting System (The operation atmospheric model run at NCEP)

GSE Garden Sprinkler Effect

GSHHS Global Self-consistent Hierarchical High-resolution Shoreline

GTS Global Telecommunication System

IFREMER French Research Institute for Exploitation of the Sea

ITCZ Intertropical Convergence Zone

JONSWAP JOint North Sea WAve Project

MEDS Marine Environmental Data Service

UKMO U.K. MetOffice

LAPS Limited Area Prediction System

MAE Mean Absolute Error

MOS Model Output Statistics

MSAS Mesoscale Surface Analysis System

NASA National Aeronautics and Space Administration

NCEP National Centers for Environmental Prediction

NCAR National Center for Atmospheric Research

NDBC National Data Buoy Center

NMC National Meteorological Center

NMOC National Meteorological Operations Centre

NOAA National Oceanic and Atmospheric Assciation

NOGAPS Navy's Operational Global Atmospheric Prediction System (The operation atmospheric model run at FNMOC)

NOPP National Oceanographic Partnership Program 
NRT Near Real Time

NWP Numerical Weather Prediction

OCF Operational Consensus Forecast

OLS Ordinary Least Squares

OSDR Operational Sensor Data Record

PDF Probability Density Function

PM Pierson-Moskowitz

$\mathbf{R}$ linear correlation coefficient

RMSE root-mean-square-error

RMSD root-mean-square-difference

SI Scatter Index

SPCZ South Pacific Convergence Zone

$H_{s}$ Significant Wave Height

$S_{s}$ Significant Wave Steepness

$U_{10} 10 \mathrm{~m}$ wind speed

USACE United States Army Corps of Engineers

SWADE Surface Wave Dynamics Experiment

SSH Sea Surface Height

SST Sea Surface Temperature

TLS Total Least Squares

UQ ULTIMATE QUICKEST

SWAMP The Sea Wave Model intercomparison Project

TP TOPEX/POSEIDON

U.S. United States

WAM WAve Model

WAM3 WAM Cycle 3

WAM4 WAM Cycle 4

WW3 WAVEWATCH III ${ }^{\circledR}$ 


\section{Appendix B}

\section{Sensitivity to Differing Definitions of Altimeter-Buoy Spatial Proximity}

Initially, co-locations were calculated based a buoy/altimeter observation proximity of $1^{\circ}$ latitude longitude instead of $50 \mathrm{~km}$. Figure B.1 shows several passes of the Jason-1 altimeter over buoy 51003 near Hawaii, where the buoy location is marked in the centre of the $1^{\circ}$ box, the western end of the horizontal lines marks the altimeter ground track, and the line length gives the measured altimeter $H_{s}$ at that point. Co-locations were determined as described in Section 4.3.3. This analysis yielded 4841 co-locations for Jason-1, and 2593 for Envisat

From these investigations using $1 \times 1^{\circ}$ boxes, it became apparent that there were differences between altimeter/buoy $H_{s}$ comparisons at low latitudes and those at high latitudes. The first column of Table B.1 shows the RMS difference between altimeter and buoy observations, for buoys above and below $45^{\circ}$ latitude separately. The RMS for both altimeters is higher at high latitudes than at low latitudes, something that is to be expected due to the climatologically higher $H_{s}$ at higher latitudes. However, the difference between RMS error at high latitudes relative to that at low latitudes is larger for Jason-1 (41\%) than it is for Envisat $(29 \%)$.

This difference is the result of the way that the spatial proximity has been defined. The width of a box defined by $1^{\circ}$ longitude changes significantly with latitude, with a box centred at $60^{\circ}$ being half the width of a box centred on the 
Appendix B. Sensitivity to Differing Definitions of Altimeter-Buoy Spatial Proximity
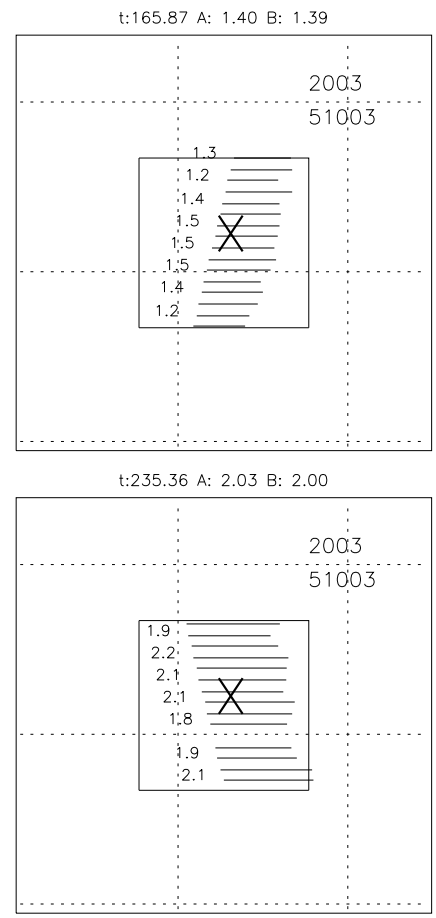

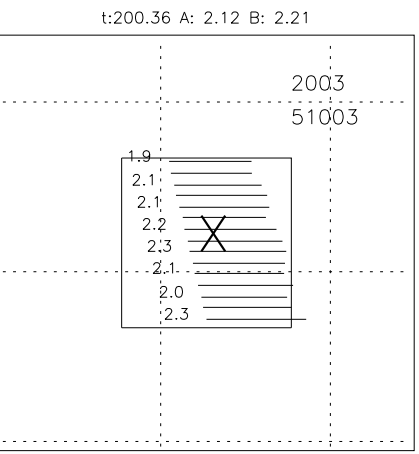

$\mathrm{t}: 235.87 \mathrm{~A}: 1.87 \mathrm{~B}: 1.86$

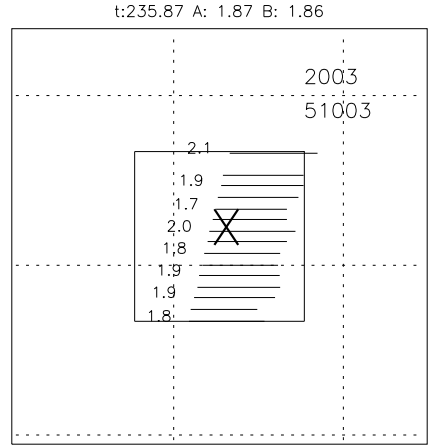

$\mathrm{t}: 200.87$ A: 1.72 B: 1.68

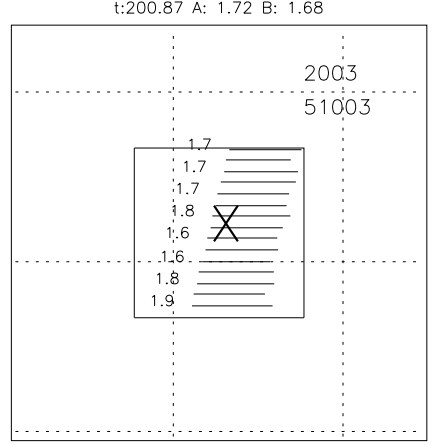

$\mathrm{t}: 305.36$ A: 1.97 B: 1.98

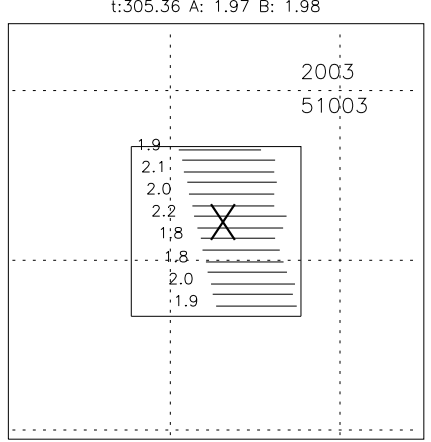

Figure B.1: Example of Jason-1 altimeter passes made over a buoy off Hawaii. Dotted grid lines are at $1^{\circ}$ intervals. The western end of the horizontal lines marks the altimeter ground track, and the line length gives the measured altimeter $H_{s}$. Julian day time of observation (t:), post processing altimeter observation (A:) and interpolated buoy observation (B:) resulting from each pass are shown

Table B.1: RMS difference (m) of altimeter data relative to buoy data for buoys above and below $45^{\circ}$ based on passes gathered using $1 \times 1^{\circ}$ boxes and $50 \mathrm{~km}$ radius circles

\begin{tabular}{lll}
\hline & $1 \times 1^{\circ}$ boxes & $50 \mathrm{~km}$ radius \\
Jason: & & \\
\hline Latitude $<45^{\circ}$ & 0.246 & 0.249 \\
Latitude $>45^{\circ}$ & 0.347 & 0.343 \\
\hline Envisat: & \\
\hline Latitude $<45^{\circ}$ & 0.223 & 0.218 \\
Latitude $>45^{\circ}$ & 0.288 & 0.311 \\
\hline
\end{tabular}


equator. As mentioned in Section 2.2, individual Jason-1 tracks have a larger zonal component than Envisat's, especially at high latitudes (Figure 2.1). As a result, this thinning of the box with latitude will affect validation results for each satellite differently.

Figure B.2 shows (a) Jason-1 and (b) Envisat tracks passing over a buoy at a latitude of $57^{\circ} \mathrm{N}$. Unlike Figure B.1, presented in cylindrical projection, the stereographic projection presented here shows the dramatically reduced width of the $1^{\circ}$ box shown around the buoy. As the altimeter continues to add parallel tracks, it is apparent from this Figure, that the maximum great arc distance between a qualifying pass and the buoy location is larger for Jason-1 than for Envisat. Hence, at high latitudes, the mean distance of a pass qualifying as a colocation will be greater for Jason-1 than for Envisat using this approach, resulting in Envisat co-locations having a relatively lower RMS.

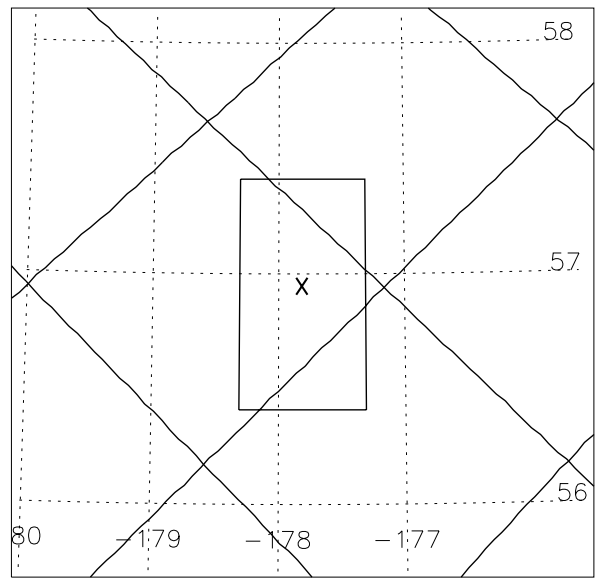

(a) Jason-1

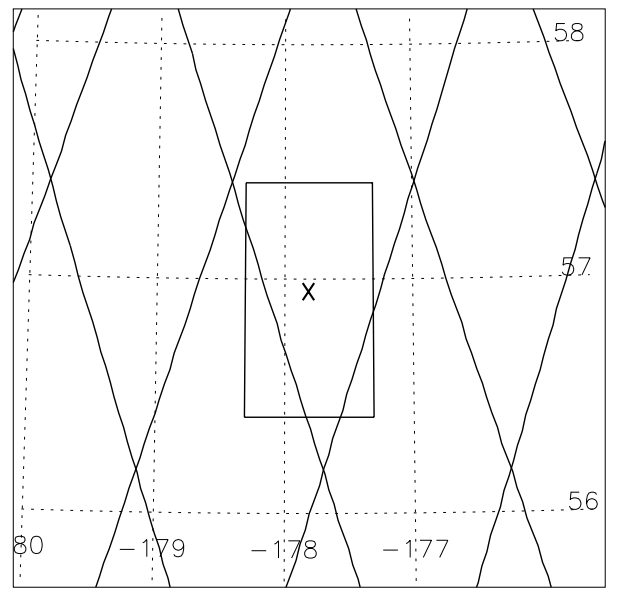

(b) Envisat

Figure B.2: Ground tracks over a buoy located near $57^{\circ} \mathrm{N}$ for (a) Jason-1 and (b) Envisat presented in stereographic projection. Surrounding the buoy is a 1 $\times 1^{\circ}$ box used to define spatial co-location limits (see text for details)

This can be addressed by defining spatial difference criteria in terms of great arc distance instead of latitude and longitude, using here a distance of $50 \mathrm{~km}$. This is equivalent to replacing the box in Figure B. 2 with a circle of radius 50 $\mathrm{km}$. Figure B.3 shows the number of valid passes occurring over a year at a range of latitudes for both $1 \times 1^{\circ}$ and $50 \mathrm{~km}$ radius circles for both altimeters. These data were determined by applying the method described above to a set of fictional buoys at $5^{\circ} \mathrm{E}$, spread between 0 and $80^{\circ} \mathrm{N}$ at $1^{\circ}$ intervals, with $5^{\circ} \mathrm{E}$ chosen to minimise land interference. The number of valid passes varied somewhat 
from buoy to buoy according to whether they were close to, or in between, track crossover points. Hence, the plot here has been smoothed by applying a 9 point running mean.

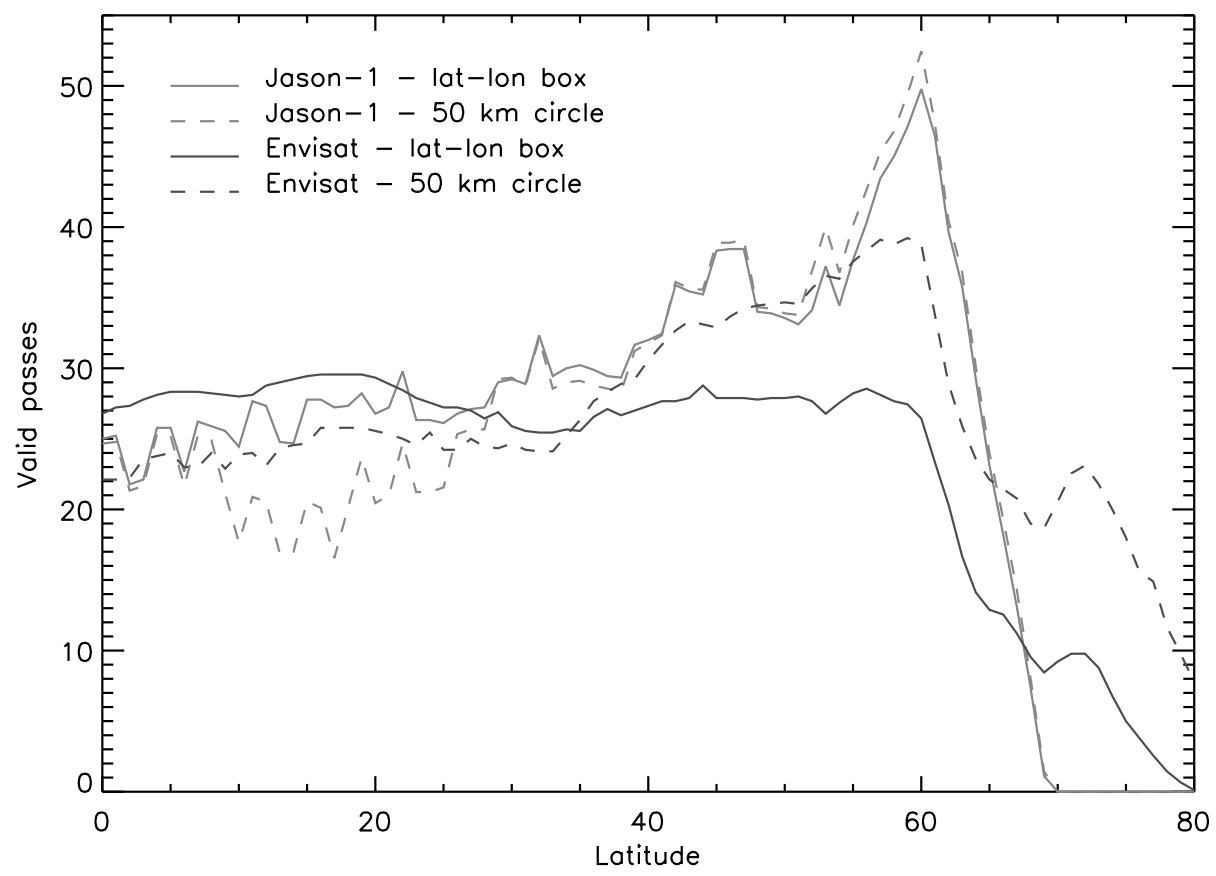

Figure B.3: Number of valid co-locations arising from buoys at different latitudes for the described method using both $\times 1^{\circ}$ boxes and $50 \mathrm{~km}$ radius circles for both Jason-1 and Envisat

The Envisat curve is smoother than that of Jason-1 due to the closer spacing of Envisat's ground tracks, resulting in less gaps in the resulting grid. For the $1 \times$ $1^{\circ}$ boxes, in the case of Jason- 1 the number of valid passes increases with latitude, reaching a peak at around $60^{\circ}$ before rapidly dropping off. This is a result of tracks becoming denser at higher latitudes. In contrast, this increase with latitude is not seen for Envisat. This is because as the tracks become closer together with latitude, the boxes also become thinner. For Jason-1, the increasingly zonal component of the tracks at high latitude make this less relevant. The peak seen in Jason- 1 at $60^{\circ}$ corresponds to the maximum extent of this satellite, at which point tracks are almost zonal, resulting in many passes through a box at this latitude. Envisat continues to produce data through to $80^{\circ}$. The minimum between 65 and $75^{\circ}$ is due to the satellite passing over land (the eastern tip of Anydr).

Of most interest here however, is the relative difference between the $1 \times 1^{\circ}$ boxes and the $50 \mathrm{~km}$ radius circles for each altimeter. For both, at low latitudes, 
circles yield fewer valid passes. $1^{\circ}$ latitude is equal to approximately $111 \mathrm{~km}$, hence, at the equator, the area of a $1 \times 1^{\circ}$ box is bigger than that of a $50 \mathrm{~km}$ radius circle. Between about 30 and 45 degrees, numbers are comparable, with circles yielding more passes at higher latitudes. Of particular note is the fact that, using $50 \mathrm{~km}$ circles produces a significant increase over $1^{\circ}$ boxes in the case of Envisat, but there is little change for Jason-1. As this increase is a result of passes at a greater distance from the buoy qualifying as a co-location, this could be expected to also produce a corresponding increase in the RMS difference.

In light of this, the final analysis used $50 \mathrm{~km}$ radius circles in place of the 1 $\times 1^{\circ}$ boxes. The second column of Table B.1 shows results using $50 \mathrm{~km}$ radius circles. RMS has increased for Envisat at high latitude, and marginally decreased at low latitude, with little difference for Jason-1, thereby eliminating this observed discrepancy between the two altimeters. Hence the use of $50 \mathrm{~km}$ circles to define co-locations is a better approach. This is especially relevant in the context of comparing the relative performance of two or more satellites with differing orbital characteristics. 
Appendix B. Sensitivity to Differing Definitions of Altimeter-Buoy Spatial Proximity 


\section{Appendix C}

\section{Buoy Details}

Table C.1: Wave buoys located around the Australian coast. Those used for verification are shown in bold.

\begin{tabular}{llrrr}
\hline Station No. & Name & Lat. & Lon. & Depth $(\mathbf{m})$ \\
\hline 52121 & Weipa & -12.68 & 141.75 & 6 \\
55014 & Bateman's Bay & -35.71 & 150.34 & 73 \\
$\mathbf{5 5 0 1 7}$ & Byron Bay & $\mathbf{- 2 8 . 6 9}$ & $\mathbf{1 5 3 . 7 3}$ & $\mathbf{7 2}$ \\
$\mathbf{5 5 0 1 8}$ & Coffs Harbour & $\mathbf{- 3 0 . 3 5}$ & $\mathbf{1 5 3 . 2 7}$ & $\mathbf{7 3}$ \\
$\mathbf{5 5 0 1 9}$ & Crowdy Head & $\mathbf{- 3 1 . 8 3}$ & $\mathbf{1 5 2 . 8 6}$ & $\mathbf{7 9}$ \\
55020 & Eden & -37.29 & 150.18 & 110 \\
55022 & Port Kembla & -34.48 & 151.03 & 78 \\
$\mathbf{5 5 0 2 4}$ & Sydney & $\mathbf{- 3 3 . 7 7}$ & $\mathbf{1 5 1 . 4 2}$ & $\mathbf{8 5}$ \\
$\mathbf{5 5 0 2 6}$ & Strahan & $\mathbf{- 4 2 . 0 8}$ & $\mathbf{1 4 5 . 0 1}$ & $\mathbf{1 0 0}$ \\
55028 & Cairns & -16.73 & 145.72 & 15 \\
55029 & Townsville & -19.17 & 147.05 & 18 \\
55031 & Mackay & -21.03 & 149.55 & 29 \\
55032 & Hay Point & -21.27 & 149.32 & 10 \\
55033 & Emu Park & $\mathbf{- 2 3 . 3}$ & 151.07 & 22 \\
55034 & Moreton Bay & -27.25 & 153.2 & 11 \\
55035 & Brisbane & $\mathbf{- 2 7 . 5}$ & 153.63 & 73 \\
55036 & Gold Coast & -27.97 & 153.45 & 18 \\
55037 & Tweed Heads & -28.18 & 153.58 & 25 \\
$\mathbf{5 5 0 3 9}$ & Kingfish B & $\mathbf{- 3 8 . 6}$ & $\mathbf{1 4 8 . 1 9}$ & $\mathbf{7 8}$ \\
$\mathbf{5 5 0 4 0}$ & Cape du Couedic & $\mathbf{- 3 6 . 0 7}$ & $\mathbf{1 3 6 . 6 2}$ & $\mathbf{8 0}$ \\
$\mathbf{5 6 0 0 2}$ & North Rankin & $\mathbf{- 1 9 . 5 9}$ & $\mathbf{1 1 6 . 1 4}$ & $\mathbf{1 2 5}$ \\
$\mathbf{5 6 0 0 4}$ & Jurien & $\mathbf{- 3 0 . 2 9}$ & $\mathbf{1 1 4 . 9 1}$ & $\mathbf{4 2}$ \\
$\mathbf{5 6 0 0 5}$ & Rottnest Island & $\mathbf{- 3 2 . 1 1}$ & $\mathbf{1 1 5 . 4}$ & $\mathbf{4 8}$ \\
$\mathbf{5 6 0 0 6}$ & Cape Naturaliste & $\mathbf{- 3 3 . 3 6}$ & $\mathbf{1 1 4 . 7 8}$ & $\mathbf{5 0}$ \\
56007 & Thevenard & $\mathbf{- 2 1 . 4 1}$ & 114.94 & 16 \\
\hline & & & &
\end{tabular}


